\title{
Cell cycle regulation by post-translational and post-transcriptional mechanisms in an anaerobic extremist - The anoxic tolerant turtle, Trachemys scripta elegans
}

\author{
Kyle Kevin Biggar \\ B.Sc. Joint Honours \\ St. Francis Xavier University, 2008
}

\begin{abstract}
A Thesis Submitted to the Faculty of Graduate Studies and Research in partial fulfillment of the requirements for the degree of
\end{abstract}

\author{
Doctor of Philosophy \\ Department of Biology
}

Carleton University

Ottawa, Ontario, Canada

(C) copyright 2013

Kyle K Biggar 
The undersigned hereby recommend to the Faculty of Graduate Studies and Research acceptance of this thesis

\title{
Cell cycle regulation by post-translational and post-transcriptional mechanisms in an anaerobic extremist - The anoxic tolerant turtle, Trachemys scripta elegans
}

\author{
submitted by \\ Kyle Kevin Biggar, B.Sc. \\ in partial fulfillment of the requirements for the degree of Doctor of Philosophy
}

Chair, Department of Biology

Thesis Supervisor

External Examiner

Carleton University 


\begin{abstract}
As a model for vertebrate long-term survival in oxygen restricted environments, the red-eared slider turtle (T. s. elegans) can adapt at the biochemical level to deal with hibernation occurring in oxygen-free (anoxic) cold water $\left(<10^{\circ} \mathrm{C}\right)$. In this thesis I hypothesized that the mechanisms which suppress ATP-expensive cell cycle activity, would contribute to establishing an hypometabolic state. To explore this possibility, this thesis studied the post-transcriptional and post-translational mechanisms of cell cycle arrest during anoxic stress in the freshwater turtle.

Results indicated a general regulation of critical cell cycle components, in addition to the possible regulation by signaling cascades (Akt/GSK-3 $\beta$ and ATR/Chk2) that are known to regulate $G_{1} / G_{0}$ phases of the cell cycle. Importantly, there is extensive regulation of Cyclin D1 protein by (1) Akt/GSK-3 $\beta$ signaling, (2) post-translational modification, (3) an AU-rich region, and (4) microRNA-induced translational suppression. This study also identified a phase-specific cell cycle arrest mechanism involving the $\mathrm{Rb}$ /E2F DNA-binding complex in both anoxic liver and kidney tissues. A novel DNA-binding complex ELISA technique was able to identify that both kidney and liver establish an $\mathrm{Rb} / \mathrm{E} 2 \mathrm{~F} 1$ mediated $\mathrm{G}_{1}$ arrest complex by 5 and $20 \mathrm{~h}$ anoxia, repectively. By $20 \mathrm{~h}$ anoxia, kidney tissue established a reversible state of $\mathrm{G}_{0}$, characterized by the prescence of a p130/E2F4 DNA-bound complex.

Overall, results from this thesis indicate that both kidney and liver enter into a $G_{1}$ arrest during anoxia. By contrast, the cell cycle in white skeletal muscle was found to be minimally regulated during anoxia and this finding is likely a reflection of its overall post-mitotic nature. Interestingly, kidney established a state of $\mathrm{G}_{1}$ arrest within $5 \mathrm{~h}$ anoxia
\end{abstract}


and subsequently transitioned to a sustainable $\mathrm{G}_{0}$ arrest by $20 \mathrm{~h}$ anoxia. However, it appears that liver $\mathrm{G}_{1}$ arrest was not established until $20 \mathrm{~h}$ anoxia. Future studies will need to explore the regulation of the cell cycle in liver after longer periods of anaerobiosis to determine whether hepatocytes are also able to transition into $\mathrm{G}_{0}$ arrest in a manner similar to kidney tissue. 


\section{ACKNOWLEDGEMENTS}

First and foremost, I would like to thank my thesis supervisor, Dr. Kenneth Storey, for taking me into his lab when I was still an ecologist and turning me into a molecular biologist. I am grateful for his scientific advice, for giving me the opportunity to try new things and for shaping me into the scientist that I have become. He has been, and will continue to be, the major scientific role model throughout my career, and I am immensely thankful for my time under his tutelage. Of course, I must also thank Jan Storey. Her expansive knowledge and continuous hard work keeps the lab running.

Unfortunately, I have been in the lab too long to list and thank all of my fellow lab members, past and present, but I would like to note that one of the most important qualities of the Storey lab is its incredible sense of community. It is a lab built on shared knowledge, and is all the more successful for it. A special thank you to both Mike Wu and Neal Dawson for our great scientific discussions - some of which, have turned into excellent scientific adventures.

Most importantly, I would like to thank my family. I have always been grateful for their constant, unwavering support and involvement throughout my life. 


\section{TABLE OF CONTENTS}

Title Page $\quad$ i

Acceptance Sheet ii

$\begin{array}{lll}\text { Abstract } & \text { iii }\end{array}$

Acknowledgements $\quad$ V

Table of Contents $\quad$ vi

List of Abbreviations vii

List of Figures viii

List of Tables $\quad$ X

List of Appendices $\quad$ xi

$\begin{array}{lll}\text { Chapter } 1 & \text { General introduction } & 1\end{array}$

Chapter 2 Characterization of cell cycle regulatory proteins 36

Chapter 3 Regulation of the $\mathrm{G}_{1}$ phase of the cell cycle 96

$\begin{array}{lll}\text { Chapter } 4 & \text { Regulation of the Rb/E2F Transcription factor complex } & 134\end{array}$

Chapter 5 Evidence of microRNA regulation of cell cycle proteins 195

$\begin{array}{lll}\text { Chapter } 6 & \text { General discussion } & 238\end{array}$

$\begin{array}{lr}\text { Appendices } & 260\end{array}$ 


\section{LIST OF ABBREVIATIONS}

AA

AEBSF

Akt

AMP, ADP, ATP

APS

ARE

ATM

ATR

BLAST

Bp

BRM

Cdc

Cdk

cDNA

Chk

CKI

$\mathrm{ddH}_{2} \mathrm{O}$

DEPC

dNTP

DTT

E2F

ECL

EDTA

ELISA

EMSA

F6P

$\mathbf{G}_{\mathbf{0}}$

$\mathbf{G}_{1}$

$\mathbf{G}_{2}$

GSK-3

HAT

HDAC

HEPES

HIF-1

HMTase

HRP

INK amino acid

4-(2-Aminoethyl) benzenesulfonyl fluoride

hydrochloride

protein kinase $\mathrm{B}$

adenosine mono-, di-, or triphosphate

ammonium persulphate

au-rich element

ataxia telangiectasia mutated

ATM-and Rad3 related

basic Local Alignment Search Tool

base pairs

Brahma

cell division cycle protein

cyclin dependent kinase

complementary deoxyribonucleic acid

checkpoint kinase

cdk inhibitor

double distilled water

diethylpyrocarbonate

deoxynucleotide triphosphate

dithiolthreitol

E2 promoter binding factor

enhanced chemiluminescence

ethylenediamine tetraacetic acid

enzyme-linked immunosorbent assay

electromobility shift assay

fructose-6-phosphate

quiescence

gap phase 1

gap phase 2

glycogen synthase kinase-3

histone acetyltransferase

histone deacetylase

$\mathrm{N}$-(2-hydroxyethyl) piperazine-(2-ethanesulfonic acid)

hypoxia-inducible factor-1

histone methyltransferase

horseradish peroxidase

inhibitor of kinase 


\begin{tabular}{|c|c|}
\hline $\mathbf{k b}$ & kilobase \\
\hline kDa & kilodalton \\
\hline Kip & kinase inhibiting protein \\
\hline LINC & Drosophila Rb/E2F and Myb complex \\
\hline $\mathbf{M}$ & mitosis \\
\hline МАРК & mitogen-activated protein kinase \\
\hline miRNA, miR & microRNA \\
\hline mRNA & messenger RNA \\
\hline MW & molecular weight \\
\hline NCBI & National Center for Biotechnology Information \\
\hline PAGE & polyacrylamide gel electrophoresis \\
\hline PBS & phosphate-buffered saline \\
\hline PCNA & proliferating cell nuclear antigen \\
\hline PCR & polymerase chain reaction \\
\hline PDK1 & phosphoinositide-dependent kinase \\
\hline PFK & 6-phosphofructokinase \\
\hline PMSF & phenylmethanesulfonyl fluoride \\
\hline PTM & post-translational modification \\
\hline PVA & poly-vinyl alcohol \\
\hline PVDF & polyvinylidine fluoride \\
\hline $\mathbf{R b}$ & retinoblastoma protein \\
\hline $\mathbf{R B P}$ & RNA-binding protein \\
\hline ROS & reactive oxygen species \\
\hline RT & room temperature $\left(\sim 21^{\circ} \mathrm{C}\right)$ \\
\hline RT-PCR & reverse-transcriptase polymerase chain reaction \\
\hline $\mathbf{S}$ & DNA synthesis/replication \\
\hline SDS & sodium dodecyl sulfate \\
\hline SOD & super-oxide dismutase \\
\hline SSC & saline-sodium citrate \\
\hline TAE & tris-acetate-ethylenediamine tetraacetic acid buffer \\
\hline TBST & tris-buffered saline containing Tween-20 \\
\hline TEMED & N,N,N',N'-tetra methylethylenediamine \\
\hline ТМB & 3,3',5,5'-Tetramethylbenzidine \\
\hline Tris & tris (hydroxymethyl) aminomethane \\
\hline UTR & untranslated region \\
\hline
\end{tabular}




\section{LIST OF FIGURES}

Figure 1.1. North American distribution of anoxia tolerant turtles, (A) T. scripta elegans and (B) C. picta bellii.

Figure 1.2. Lactate movement into a calcium carbonate rich shell during anoxia in the hypometabolic turtle.

Figure 1.3. Transitions to and from a hypometabolic state in the anoxic turtle. Upon initial hypoxic sensing, cellular adjustments occur to reprioritize ATP metabolism and defend the cell from oxidative damage upon oxygen reperfusion.

Figure 1.4. Generalized signaling pathways of ERK, SAPK/JNK, and p38 including their influences on cell functions.

Figure 1.5. General mechanism of translational regulation by microRNA. MicroRNAs are targeted to the 3' UTR of specific mRNA transcripts.

Figure 2.1. Expression profiles of Cyclin/Cdk complexes throughout the cell cycle. Cyclic expression of these complexes allow for the completion of one phase before the initiation of the subsequent phase of the cell cycle.

Figure 2.2. Regulation of Cyclin D1 degradation by Akt/PI3K signaling and regulation of GSK-3 $\beta$ activity.

Figure 2.3. The $\mathrm{Rb}$ /E2F pathway. Sequential phosphorylation by kinase complexes Cyclin D1/Cdk4:Cdk6 and Cyclin E1/Cdk2, respectively, causes conformational changes to the $\mathrm{Rb}$ structure and release of $\mathrm{E} 2 \mathrm{~F}$. The release of E2F is necessary for the expression of S-phases genes.

Figure 2.4. Image files for immunoblotting and RT-PCR targets in control and anoxic (5 and $20 \mathrm{~h}$ ) conditions from (A) liver, (B) kidney and, (C) white skeletal muscle tissue from $T$. scripta elegans.

Figure 2.5. Effect of 5 and $20 \mathrm{~h}$ of anoxic submergence on relative protein and phosphorylation levels of Akt in T. s. elegans.

Figure 2.6. Effect of 5 and $20 \mathrm{~h}$ of anoxic submergence on relative protein and phosphorylation levels of GSK-3b and phosphorylation of its downstream target, Cyclin D1 in T. s. elegans.

Figure 2.7. Effect of 5 and $20 \mathrm{~h}$ of anoxic submergence on relative protein levels of Cyclins (types D1, E1, A1 and B1) in T. s. elegans.

Figure 2.8. Effect of 5 and $20 \mathrm{~h}$ of anoxic submergence on relative nuclear protein levels of Cyclins (types D1, E1, A1 and B1) in T. s. elegans.

Figure 2.9. Effect of 5 and $20 \mathrm{~h}$ of anoxic submergence on transcript levels of Cyclins (types D1, E1, A1 and B1) in T. s. elegans.

Figure 2.10. Nucleotide and deduced amino acid sequence for T. s. elegans partial cyclin $d 1$ sequence.

Figure 2.11. Nucleotide and deduced amino acid sequence for T. s. elegans partial cyclin el sequence.

Figure 2.12. Nucleotide and deduced amino acid sequence for T. s. elegans partial cyclin al sequence.

Figure 2.13. $\quad$ Nucleotide and deduced amino acid sequence for T. s. elegans partial cyclin b1 sequence.

Figure 2.14. Effect of 5 and $20 \mathrm{~h}$ of anoxic submergence on relative protein and phosphorylation levels of Cdks (types 4, 6,2) and Cdc2 in T. s. elegans.

Figure 2.15. Effect of 5 and $20 \mathrm{~h}$ of anoxic submergence on relative nuclear levels of Cdks (types 4, 6, 2) and Cdc2 in T. s. elegans.

Figure 2.16. Effect of 5 and $20 \mathrm{~h}$ of anoxic submergence on transcript levels of Cdks (types 4, 6, 2) and Cdc2 in T. s. elegans.

Figure 2.17. Nucleotide and deduced amino acid sequence for $T$. s. elegans partial $c d k 4$ sequence.

Figure 2.18. Nucleotide and deduced amino acid sequence for T. s. elegans partial $c d k 6$ sequence. 
Figure 2.19. Nucleotide and deduced amino acid sequence for T. s. elegans partial $c d k 2$ sequence.

Figure 2.20. Nucleotide and deduced amino acid sequence for $T$. s. elegans partial $c d c 2$ sequence.

Figure 3.1. $\quad$ Proposed hypoxia induced regulation of the cell cycle.

Figure 3.2. The ATM/ATR pathway. Stress activation of either ATM and ATR results in downstream phosphorylation of checkpoint kinases (Chk1/2) and regulation of cell cycle effectors.

Figure 3.3. Image files for immunoblotting and RT-PCR targets in control and anoxic (5 and $20 \mathrm{~h}$ ) conditions from (A) liver, (B) kidney and, (C) white skeletal muscle tissue from T. scripta elegans.

Figure 3.4. Effect of 5 and $20 \mathrm{~h}$ of anoxic submergence on relative protein expression of ATM and ATR in T. s. elegans.

Figure 3.5. Effect of 5 and $20 \mathrm{~h}$ of anoxic submergence on relative protein and phosphorylation levels of Chk1 and Chk2 in T. s. elegans.

Figure 3.6. Effect of 5 and $20 \mathrm{~h}$ of anoxic submergence on relative protein expression of Cdc25a and Cdc25c in T. s. elegans.

Figure 3.7. Effect of 5 and $20 \mathrm{~h}$ of anoxic submergence on relative protein and phosphorylation levels of CKIs (types p27, p16 and p21) in T. s. elegans.

Figure 3.8. Effect of 5 and $20 \mathrm{~h}$ of anoxic submergence on transcript levels of $p 27$ in T. s. elegans.

Figure 3.9. $\quad$ Nucleotide and deduced amino acid sequence for T. s. elegans partial $p 27$ sequence.

Figure 4.1. The Rb/E2F pathway. Sequential phosphorylation by kinase complexes Cyclin D1/Cdk4:Cdk6 and Cyclin E1/Cdk2, respectively, causes conformational changes to the $\mathrm{Rb}$ structure and release of E2F. The release of E2F is necessary for the expression of S-phases genes.

Figure 4.2. Image files for immunoblotting and RT-PCR targets in control and anoxic (5 and $20 \mathrm{~h}$ ) conditions from (A) liver, (B) kidney and, (C) white skeletal muscle tissue from $T$. scripta elegans.

Figure 4.3. Effect of 5 and $20 \mathrm{~h}$ of anoxic submergence on relative protein expression of E2F1 and E2F4 in T. s. elegans.

Figure 4.4. Effect of 5 and $20 \mathrm{~h}$ of anoxic submergence on relative nuclear protein expression of E2F1 and E2F4 in T. s. elegans.

Figure 4.5. Effect of 5 and $20 \mathrm{~h}$ of anoxic submergence on relative protein and phosphorylation levels of $\mathrm{Rb}$ in $T$. s. elegans.

Figure 4.6. Effect of 5 and $20 \mathrm{~h}$ of anoxic submergence on relative nuclear protein and phosphorylation levels of $\mathrm{Rb}$ in $T$. s. elegans.

Figure 4.7. Effect of 5 and $20 \mathrm{~h}$ of anoxic submergence on relative protein expression of p130 in T. s. elegans.

Figure 4.8. Effect of 5 and $20 \mathrm{~h}$ of anoxic submergence on the relative nuclear protein expression of $\mathrm{p} 130$ in $T$. s. elegans.

Figure 4.9. Effect of 5 and $20 \mathrm{~h}$ of anoxic submergence on relative protein expression of epigentic modifying protein, Suv29H1 and HDAC4, in T. s. elegans.

Figure 4.10. Effect of 5 and $20 \mathrm{~h}$ of anoxic submergence on the relative nuclear protein expression of epigentic modifying protein, Suv29H1 and HDAC4, in T. $s$. elegans.

Figure 4.11. Electrophoretic mobility shift assay (EMSA) for the E2F family of transcription factors. 
Figure 4.12. Effect of unlabeled DNA probe competition on the relative binding of E2F1 from control liver tissue samples from T. s. elegans. Either unlabeled DNA probe (Wild-type) or an unlabeled DNA probe with a mutated DNA binding site (Mutant) were incubated with total protein in the presence of biotin-labeled probe.

Figure 4.13. Effect of 5 and $20 \mathrm{~h}$ of the relative DNA binding activity of E2F1 and E2F4 in T. s. elegans.

Figure 4.14. Effect of 5 and $20 \mathrm{~h}$ of the relative protein complex composition containing E2F bound to DNA in T. s. elegans. E2F bound proteins include Rb, p130, Suv39H1 and HDAC4.

Figure 4.15. Effect of 5 and $20 \mathrm{~h}$ of anoxic submergence on the (A) mRNA and (B) protein expression levels of the downstream E2F target, PCNA, in T. $s$. elegans.

Figure 4.16. Nucleotide and deduced amino acid sequence for T. s. elegans partial pcna sequence.

Figure 4.17. Effect of 5 and $20 \mathrm{~h}$ of anoxic submergence on transcript levels of $e 2 f 4, r b$ and 130 in T. s. elegans.

Figure 4.18. Nucleotide and deduced amino acid sequence for T. s. elegans partial e2f4 sequence.

Figure 4.19. $\quad$ Nucleotide and deduced amino acid sequence for T. s. elegans partial $r b$ sequence.

Figure 4.20. Nucleotide and deduced amino acid sequence for T. s. elegans partial p130 sequence.

Figure 5.1. MicroRNA biogenesis. MicroRNAs begin in the nucleus where they are processed and exported into the cytoplasm.

Figure 5.2. Schematic representation of microRNA function. Mature microRNAs regulate protein translation by the 3' UTR of a conserved target mRNA.

Figure 5.3. Effect of $\mathrm{p} 53$ and its downstream targets (p21, miR-16-1, miR-15a and miR-34a) on the cell cycle.

Figure 5.4. Effect of 5 and $20 \mathrm{~h}$ of anoxic submergence on relative protein, phosphorylation and mRNA levels of Cyclin D1 in T. s. elegans.

Figure 5.5. Identification of regulatory elements within the cyclin d1 3' UTR.

Figure 5.6. Theoretical binding of tsc-miRNA-16-1, tsc-miR-15a and $t s c-m i R-34 a$ to conserved binding regions in the cyclin D1 3' UTR of the turtle.

Figure 5.7. Theoretical binding of $t s c-m i R N A-16-1, t s c-m i R-15 a$ and $t s c-m i R-34 a$ to conserved binding regions in the cyclin D1 3' UTR of the turtle.

Figure 5.8. Effect of anoxic stress on the relative expression of Dicer protein in the liver, kidney and skeletal muscle from control and anoxic (5 and $20 \mathrm{~h}$ ) turtles.

Figure 5.9. Effect of anoxic stress on the relative expression of $t s c-m i R-16-1$ in the liver, kidney and skeletal muscle from control and anoxic (5 and $20 \mathrm{~h})$ turtles.

Figure 5.10. Effect of anoxic stress on the relative expression of $t s c-m i R-15 a$ in the liver, kidney and skeletal muscle from control and anoxic (5 and $20 \mathrm{~h})$ turtles.

Figure 5.11. Effect of anoxic stress on the relative expression of $t s c-m i R-34 a$ in the liver, kidney and skeletal muscle from control and anoxic (5 and $20 \mathrm{~h})$ turtles.

Figure 5.12. Effect of anoxic stress on the relative abundance of poly-adenylated mRNA from the liver, kidney and skeletal muscle of control and anoxic (5 and $20 \mathrm{~h}$ ) turtles. 


\section{LIST OF TABLES}

Table 5.1. Determination of RNA binding structure and theoretical minimum free energy (mfe) values using RNAhybrid. Shaded regions indicate the conserved seed-pairing between the microRNA and its target. 


\section{LIST OF APPENDICES}

$\begin{array}{ll}\text { Appendix A } & \text { List of publications } \\ \text { Appendix B } & \text { Communications at scientific meetings } \\ \text { Appendix C } & \text { List of antibodies } \\ \text { Appendix D } & \text { List of immunoblotting conditions } \\ \text { Appendix E } & \text { RNA integrity } \\ \text { Appendix F } & \text { List of primers } \\ \text { Appendix G } & \text { List of annealing temperatures } \\ \text { Appendix H } & \text { List of microRNA amplification primers } \\ \text { Appendix I } & \text { Ensuring antibody specificity for immunoblotting } \\ \text { Appendix } \mathbf{J} & \text { Experimental optimization for DNA-binding complex ELISA } \\ \text { Appendix K } & \begin{array}{l}\text { Experimental method and validation of mature microRNA } \\ \text { amplification }\end{array}\end{array}$




\section{CHAPTER 1}

\section{GENERAL INTRODUCTION}

"I look at this turtle now in my hand and wonder what grace allows me to hold and ponder such a tangible piece of the history of life on Earth."

- David M. Carroll, 1991, The Year of the Turtle 


\subsection{Environmental Oxygen}

All the oxygen in the present atmosphere is believed to have had a biological origin, and was mostly formed between 3,000,000,000 and 1,000,000,000 years ago (Embley and Martin, 2006). The production of gaseous oxygen is thought to be a result of photosynthesis by cyanobacteria and the earliest green plants. It was only at this time that primitive eukaryotes acquired mitochondria, and life forms evolved to use oxygen as the final acceptor in their electron transport pathways (Embley and Martin, 2006). The high redox potential of oxygen allows it to easily accept electrons from reduced substrates, making it extremely useful for energy production. The ability to extract greater amounts of energy from organic molecules, by using oxygen-linked catabolism, has allowed highly complex life forms to evolve and has made oxygen vital to most life on Earth. However, this extreme dependence on oxygen comes at a cost because many animals then became highly dependent upon oxygen and highly sensitive to oxygen deprivation. Mammalian organ systems are designed to function under high oxygen content and every effort is made to maintain the oxygen level within a narrow range of operating limits. Situations of hypoxia or anoxia can rapidly lead to severe tissue damage and inevitable death to intolerant organisms (Brierley, 1977).

Living animals are constantly faced with various environmental stresses that challenge normal life, including; oxygen limitation, very low or high temperatures, water limitation and food restriction (Storey and Storey, 2010; Storey and Storey, 2004a). Of these stresses, oxygen variation in the environment is one that many animals commonly experience. For example, in their northern ranges, freshwater turtles can experience a wide variation in oxygen supply, arising by either; (1) variations in environmental oxygen availability (e.g. ice-locked ponds and lakes with hypoxic or anoxic water) that 
deny turtles access to air to breathe for extended periods of the time or, (2) by animal behaviors that interrupt the supply of oxygen (e.g. extended periods of breath-hold diving) (Storey, 2007) (Figure 1.1). Depending on their length and severity, both of these situations decrease oxygen supply and lead to an inhibition of oxidative phosphorylation and subsequent interruption in mitochondrial ATP production (Storey, 2007). The decrease in ATP production can quickly lead to a disruption of many ATP-utilizing processes in the cell. Reduced energy availability for ATP-dependent ion channels can be particularly damaging since this disrupts the normal balance between the opposing rates of ATP-dependent ion pumps versus passive ion channels (ATP-independent), resulting in a quick loss of membrane potential difference (Hochachka and Lutz, 2001). In the brain, this loss of membrane potential causes a rapid breakdown of critical transmembrane ion gradients, a rise in intracellular $\mathrm{Ca}^{2+}$ concentrations, and a release of excitatory neurotransmitters (Fraser et al., 2001). It is this release of neurotransmitters and increase of intracellular $\mathrm{Ca}^{2+}$ that triggers multiple dangerous effects including the signaling of programmed cell death (or apoptosis) (Orrenius et al., 2003).

Apart from turtles, most other animals experience situations of oxygen limitation or deprivation that occur either due to variations in environmental oxygen levels, or behaviors that interrupt oxygen supply. As a result, most animals have developed mechanisms that allow them to compensate for mild or short-term hypoxia. In mammals, these responses are activated in order to; (1) improve oxygen delivery to tissues and, (2) increase anaerobic ATP production to compensate for the reduced ATP output from oxidative phosphorylation. Physiological responses to low oxygen include increases in ventilation and lung gas exchange, an increase in hemoglobin unloading of oxygen, and 
release of stored red blood cells from the spleen (Storey and Storey, 2004b). These adaptations serve to increase $\mathrm{O}_{2}$ uptake, delivery to organs, and carrying capacity. The normal metabolic response to oxygen deprivation includes an increase in the glycolytic rate, as well as consumption of creatine phosphate reserves (Storey and Storey, 2004b). Often these physiological/biochemical compensatory adjustments are sufficient to deal with short term or mild hypoxia/anoxia but prolonged or severe oxygen deprivation is still lethal to many animals.

\subsection{Facultative anaerobiosis: anoxia survival in turtles}

The mammalian focus on maintaining optimal oxygen supply to organs (in order to maintain high rates of aerobic metabolism) is not universal across the animal kingdom. Many organisms, including a variety of ectothermic vertebrates, can live without oxygen for extended periods of time, functioning as facultative anaerobes (Storey, 2007; Nilsson and Rensshaw, 2004). For example, lack of oxygen is a daily occurrence for many species of turtles which spend much of their lives underwater either diving for food or escaping predation (Clark and Miller, 1973; Jackson, 1968). Winter survival for many freshwater turtles is also ensured by underwater brumation, providing an escape from freezing temperatures.

For short-term oxygen deprivation, such as diving for food, anaerobic metabolism can easily meet metabolic demands (Storey and Storey, 2004b). However, for long term survival, such as overwintering, turtles turn to one of two modes of survival; (1) extrapulmonary mechanisms of oxygen uptake, and (2) a decrease in metabolic demand (hypometabolism). For most turtles, a capacity to survive underwater without pulmonary 
ventilation stems in part from their proficiency in exchanging respiratory gases with water across well-vascularized epithelium linings of the throat and/or cloaca (Ultsch, 1985; Storey and Storey, 2004b). This method of gas exchange, known as extrapulmonary oxygen uptake, is most often utilized by soft-shelled turtles (Reese et al., 2003). For example, many subtropical Australia turtles can utilize extrapulmonary ventilation to obtain adequate oxygen supply even in warm water (where dissolved $\mathrm{O}_{2}$ is low) (Gordos and Franklin, 2002). However, other turtles strongly reduce their metabolic rate and lower oxygen demand to survive for several months underwater while overwintering and have perfected facultative anaerobiosis.

The best facultative anaerobes among freshwater turtles are members of the genera Trachemys (pond slider turtles) and Chrysemys (painted turtles). These species are widespread over most of the United States and southern Canada (Figure 1.1). Sliders and painted turtles are able to survive without oxygen for up to two weeks at $\sim 16^{\circ} \mathrm{C}$ and for 12 to 18 weeks at $\sim 3^{\circ} \mathrm{C}$ (Ultsch, 1985; Ultsch and Jackson, 1982; Herbert and Jackson, 1985; Gatten, 1987). By comparison, mammalian tissues are notoriously sensitive to even brief episodes of anoxia; for example, humans can endure only a few minutes of anoxia without irreversible brain damage. Turtles, however, employ several strategies to survive oxygen lack including a rapid reduction of metabolic rate to just $\sim 10-20 \%$ of normoxic resting rate, and buffering the lactic acid produced by anaerobic glycolysis in their bony shell (Jackson, 1968; Herbert and Jackson, 1985). 


\section{Previous research has documented four fundamental defense strategies}

\section{utilized by turtles and other anoxia tolerant organisms:}

(1) The physiological responses to low oxygen and the cellular transition to the anoxic state. This includes mechanisms of hypoxia sensing and signal transduction, the regulation of neurotransmitters, ion channels, and metabolic reorganization.

(2) Suppressing energy metabolism (with coordinated suppression of energy demand) as a means to balance ATP production with ATP utilization, thus avoiding the consequences of energy failure during hypometabolism.

(3) Minimizing the damage caused by oxygen reperfusion during reoxygenation after anoxia.

(4) Utilizing signaling pathways as a means to rapidly detect low oxygen stress, allowing cellular alterations and protection mechanisms to promote survival.

Recent research has also identified additional molecular strategies of hypometabolism that protect anoxia-tolerant turtles (e.g. unfolded protein response and antioxidant defense) and highlighted emerging areas of research into the mechanisms of global metabolic control (post-translational and post-transcriptional) when oxygen is limited.

The overall focus of this thesis builds upon the existing research on the biochemical and physiological responses that support anoxia-induced metabolic rate depression in the red-eared slider turtle (Trachemys scripta elegans). 


\subsection{Hypometabolism in the turtle}

Upon encountering hypoxic conditions, the first response of the turtle is to enhance oxygen delivery and the oxygen extraction systems. These mechanisms are well established in the literature and include the physiological responses of increasing lung ventilation, alterations to hemoglobin oxygen affinity leading to enhanced oxygen extraction, as well as, increasing cardiac output to improve oxygen delivery to organs (Lutz and Storey, 1997; Jackson, 2002). If oxygen concentrations continue to fall, the systemic alterations to oxygen extraction quickly become inadequate to supply enough oxygen to deprived tissues. When this occurs, oxygen-independent metabolic pathways, such as anaerobic glycolysis, are fully recruited and are followed by regulatory actions that reduce oxygen demand by cells (Lutz and Storey, 1997; Jackson, 2002; Hochachka et al., 1996). This introduces a very important issue -- increasing the rate of glycolysis does increase ATP output, but also results in a quick depletion of internal fuel reserves, as well as a large accumulation of acidic end products (Kelly and Storey, 1988; Jackson, 1997). Turtles must have strategies to cope with this problem. One way to do so is to enhance tolerance of anaerobic end product buildup. Freshwater turtles, such as the painted turtle (C. picta bellii), can accumulate plasma lactate concentration as high as 150-200 mM after several winter months submerged in cold water (Ultsch and Jackson, 1987; Jackson et al., 2000; Jackson et al., 2007). In comparison, a human exercised to

exhaustion may only experience an extreme plasma lactate level of 20-25 mM (Jackson, 2011). The acid load associated with lactate accumulation far exceeds the natural buffering capacity of the plasma bicarbonate system in turtles $(35-45 \mathrm{mM})$. The turtle is able to cope with these extraordinarily high lactate levels by utilizing key physiological 
resources, namely its calcium carbonate-rich shell and skeleton (Jackson et al., 2000; Jackson et al., 2007). Carbonate minerals are dissolved into the extracellular fluid and form complexes with protons and lactate anions to supplement buffering. In addition, lactate is taken up by both the shell and bone, where natural carbonate acts to buffer and store lactate until normoxic conditions are restored (Figure 1.2) (Jackson et al., 2000; Jackson et al., 2007).

When oxygen supplies are completely cut off (anoxia), ATP demand soon outstrips ATP supply and neural cell death is inevitable for intolerant animals. If the decrease in ATP production is not corrected, an imbalance between ATP-dependant ion pumps and passive ion channels is created and as a result, membrane potential difference collapses (Doll et al., 1991). Once a collapse of membrane potential has occurred, there is a large influx of $\mathrm{Ca}^{2+}$ through plasma membrane channels and a variety of irreversible destructive events, such as apoptosis, are initiated (Hajnoczky et al., 2003). Additionally, increasing ATP supply via anaerobiosis, quickly consumes substrate and inevitably serves only to shorten survival time. Thus, it is the reduction ATP demand, not an increase in glycolysis that is the only viable long-term strategy for a vertebrate to survive without oxygen.

Turtles escape anoxia-induced death by suppressing, rebalancing and reprioritizing the rates of ATP-utilizing and ATP-generating processes so that they can sustain long term viability without oxygen (Storey and Storey, 2007). Both ATP production and ATP consumption are strongly decreased in response to anoxia in tolerant turtles. For example, studies with isolated turtle hepatocytes showed a 94\% decrease in overall ATP turnover when exposed to anoxic conditions (Hochachka et al., 1996). 
Dramatic changes were seen in the portion of ATP turnover that was devoted to five main ATP consuming processes: (1) ion motive ATPases, (2) protein synthesis, (3) protein degradation, (4) gluconeogenesis, and (5) urea synthesis. As a result, the reorganization of metabolic processes allows turtle organs to direct the available energy into the vital processes for cellular survival under anoxia. This results in the most efficient ATP utilization under energy-limited conditions, and is the hallmark of hypometabolism in many organisms, including turtles. As reported by Hochachka and colleagues (1996), the main features of anoxia survival via hypometabolism include:

(1) An oxygen sensing mechanism and signal transduction pathways that communicate the hypoxic/anoxic transitions to cells

(2) A set of genes that are up-regulated

(3) A set of genes that are down-regulated

(4) A decrease in ATP utilization by non-essential pathways

(5) A decline in membrane permeability and impulse frequency in neural tissues, and

(6) A sustainable balance of ATP utilization and production.

These are the fundamental, and highly regulated, processes that allow turtles to survive extended periods of reduced oxygen in a hypometabolic state (Figure 1.3) (Hochachka et al., 1997). Indeed, in turtles, a profound metabolic rate depression to only $10-20 \%$ of the corresponding aerobic resting rate, at the same temperature, occurs in response to anoxia (Storey and Storey, 1990). 


\subsection{Transitions to and from the hypometabolic state}

Metabolism is dynamic, and all living organisms must respond to a wide variety of needs, signals and stresses in order to remain alive. Most often these responses adjust metabolism to meet new demands or to cope with internal or external stressors.

Considerable research on metabolic depression in turtles, has defined crucial, and highly regulated, transitions to and from the hypometabolic state (Hochachka et al., 1996; Lutz and Milton, 2004). These transitions include: (1) an initial downregulation of ATP utilizing processes during the transition between hypoxia and anoxia, (2) long-term maintenance in a metabolically depressed state, and (3) a rapid upregulation of metabolic rate and normal cellular activity when oxygen becomes available (Figure 1.3).

Entrance into hypometabolism involves a strong reduction of ATP use during the first 1-2 hours of hypoxia, so that ATP demand can be satisfied through anaerobic glycolysis (Figure 1.3) (Brooks and Storey, 1989). The transition to the hypometabolic state must be highly regulated in order to achieve a coordinated suppression of many metabolic functions and a new balance between ATP-producing and ATP-utilizing reactions in cells. A failure to meet cellular ATP requirements during this period is the difference between long-term survival and catastrophic cell death. Regulation of this entry phase is highly coordinated through rapid post-translational modifications of cellular enzymes and signaling pathways (Storey and Storey, 2004). Reversible protein phosphorylation in turtles by the action of protein kinases and protein phosphatases has been shown to be the most widespread and potent mechanism in controlling hypometabolism, proceeding at a rapid rate (Storey, 2007; Brooks and Storey, 1993; Greenway and Storey, 2000). However, when arterial $\mathrm{O}_{2}$ tension drops below a critical 
limit (arterial $\mathrm{pO}_{2}$ of 20 Torr in turtles), a long-term strategy to conserve ATP supply is initiated (Hochachka, 1988).

The second phase of the hypometabolic transition in turtles is entry into a maintenance period (Figure 1.3). This is the longest hypometabolic period in the turtle and can last anywhere from hours (long dives) to days or months (overwintering). It involves the maintenance of cellular processes and reestablishment of homeostasis at an order of magnitude lower than the normoxic state. Research in recent years has also started to define mechanisms that regulate an overall strong suppression of transcription and translation, while enhancing the expression of selected genes and proteins with protective function (Storey and Storey, 2004b; Land and Hochachka, 1994; Storey, 2004). Much of the research to date, focusing on the maintenance of hypometabolism in turtles, has been concerned with energetics, fuel catabolism, and molecular controls on energy-expensive cell functions such as controls on gene transcription, protein translation, and neuronal ion-motive pumps and channels. The present thesis continues this focus with a multifaceted examination of the regulation of another energy-expensive function, the cell cycle.

The final transition phase is the exit from hypometabolism, or recovery, once normoxic conditions have been restored (Figure 1.3). Similar to the transition into hypometabolism, once oxygen is again available there must be an equally regulated reactivation of suppressed cellular activities and processes. However, transitioning back to the normoxic state is not as simple as reactivating each suppressed process. Within the first 10 min of recovery, blood oxygen rapidly returns to normoxic levels (Willmore and Storey, 1997). The reintroduction of oxygen to the turtle brings about a flood of 
damaging reactive oxygen species (ROS) and protective mechanisms must be in place to deal with these. Damage resulting from uncontrolled generation of ROS can lead to peroxidation of polyunsaturated fatty acids in membranes and lipid depots as well as the oxidation of sulfhydryl-containing enzymes, carbohydrates (polysaccharide depolymerization) and nucleic acids (single and double strand scissions) (Tribble et al., 1987; Blokhina et al., 2003). To deal with the potential for ROS induced cellular damage, T. scripta elegans maintains high constitutive activities of various antioxidant enzymes including catalase, superoxide dismutase (SOD) and alkyl hydroperoxide reductase (Willmore and Storey, 1997). In order to survive each of these transitional phases to and from the hypometabolic state, turtle must successfully negotiate the separate requirements needed at each step. The hypometablic transition requires a highly controlled regulation at both the physiological and molecular levels.

\subsection{Metabolic demand: satisfying ATP homeostasis with hypometabolism}

Organisms utilizing aerobic metabolism are able to make use of carbohydrates, lipids, and proteins as oxidative fuels. However, when oxygen becomes limiting, organisms are restricted almost solely to the use of carbohydrates as the major fermentable fuel. This means that the major fuel reserve for most animals, lipids, becomes unusable when oxygen levels fall. Since ATP generation from glycolysis is extremely low (a net of only 2 mol ATP per mol of glucose catabolized), it is obvious that ATP supply would soon fall short of ATP demand in a very short period. Hence, in order to maintain normal levels of ATP generation (aerobic ATP generation yields 36 mol ATP per mol of glucose catabolized), organisms would have to burn 18-times more 
glucose in the absence versus presence of oxygen. This has disastrous consequences for intolerant animals, since glucose stores are essentially wasted while generating high accumulation of acidic waste products (lactate ions and associated protons) (Jackson, 2002). Successful facultative anaerobes, such as turtles, must put in place particular adaptations that allow them to survive the negative consequences of running anaerobic glycolysis. Such mechanisms include: (1) increasing reserves of fermentable fuels (glycogen), particularly in the liver, (2) a tolerance for large changes in the $\mathrm{pH}$ of both intra- and extra-cellular fluids, and (3) a means to depress metabolic demand such that ATP supply can be adequately matched with low levels of glycolysis. During the early hours of the hypoxic transition, creatine phosphate reserves, in combination with glycolysis, can contribute substantially to ATP needs in tissues that have high phosphagen reserves. However, these reserves are quickly depleted and are only able to contribute within the early hours of hypoxia so alternate mechanisms for ATP homeostatsis must be put into place (Clark and Miller, 1973; Lutz et al., 1984).

\subsection{Detection of extracellular stress: post-translational modification}

As previously alluded to, one of the most widely researched mechanisms of metabolic rate depression is the control of protein/enzyme activity via reversible protein phosphorylation (Storey and Storey, 2004b). This mechanism reappears across phylogeny as the means of making major changes in metabolic rate and reorganizing metabolism in numerous animal models, including estivating snails (O. lactea), frozen frogs $(R$. sylvatica), anoxic turtles (T. scripta elegans) and hibernating squirrels ( $S$. tridecemlineatus) (Brooks and Storey, 1993; Brooks and Storey, 1995; MacDonald and 
Storey, 1999; Dieni and Storey, 2009). Through the use of ${ }^{32} \mathrm{P}$, one study documented an increase in global phosphorylated protein levels during anoxic exposure in T. scripta elegans (Brooks and Storey, 1993). Protein phosphorylation patterns during anoxia revealed 1.6-, 2.4-, and 1.3-fold increases in ${ }^{32} \mathrm{P}$ incorporation in anoxic brain, heart, and liver, respectively.

Reversible phosphorylation is also responsible for the detection of extracellular stimuli through signal transduction networks. Growing interest in the intracellular signaling of anoxia in turtles has focused on the differential regulation of the mitogenactivated protein kinase (MAPK) superfamily. Phosphorylation of a MAPK family member results in a conformational change in protein structure and a $>1000$-fold increase in kinase activity (Hoeflich and Woodgett, 2001). In effect, MAPKs are not functional as signaling molecules until phosphorylated by their respective upstream kinases. Once activated, MAPKs phosphorylate their downstream proteins, many of which are transcription factors which have key roles in the up-regulation of genes critical to the anoxia stress response in the turtle (Figure 1.4) (Cowan and Storey, 2003). The three main MAPK family members (ERK, JNK, p38), and their regulation in the anoxic turtle, are briefly summarized in the section below.

Studies of MAPK activation in response to anoxia in adult and hatchling $T$. scripta elegans have identified one common result; both ERK and p38 have little or no involvement in the hypometabolic responses to anoxia in turtles (Greenway and Storey, 2000). By contrast, the activity of JNK increased in tissues of both hatchling and adult turtles in response to anoxia. In T. scripta elegans, JNK showed maximum activation after $5 \mathrm{~h}$ of anoxia but quickly decreased with increased exposure. This result suggests 
that JNK may have a role in the hypoxic transition period into anoxia with JNK reduced back to control values when a complete depression of metabolic rate has been achieved. It has been suggested that the lack of involvement of the ERK pathway could be a result of its primary response to growth factors (Johnson and Lapadat, 2002). Growth factors are responsible for stimulating cell growth, proliferation, and cell differentiation, processes which under anoxia would lead to a rapid depletion of cellular ATP supply, and inevitably, cell death. The unchanged p38 in the anoxic turtle is of particular interest as it contrasts with models of anoxia intolerance. It has been shown that by transiently activating the p38 pathway, through short preconditioning exposures to ischemia in mammalian heart, that the recovery of function during reperfusion is significantly improved (Marais et al., 2001). Additionally, activation of both JNK and p38 has been correlated with improved survival of ischemia-reperfusion in mammalian kidney (Park et al., 2001). It has been speculated that the metabolic responses to anoxia of tolerant versus intolerant organisms may be mediated through the differential regulation seen in the p38 signal transduction pathway; albeit more research is necessary to reach such a conclusion (Cowan and Storey, 2003). The patterns of MAPK activation in a stress-tolerant animal suggest the relative importance of these kinase pathways in cellular adaptation to oxygen deprivation and identify novel natural activators of MAPKs in vivo.

\subsection{Detection of extracellular stress: post-transcriptional control}

The suppression of protein synthesis during metabolic rate depression is vital to anoxic survival in turtles. Protein synthesis consumes a very large portion of available ATP turnover in cells under normoxic conditions, since about 5 ATP equivalents are 
needed per peptide bond formed (Cramer et al., 1991). Some freshwater turtles have been shown to decrease the rate of ATP use by protein synthesis to only $\sim 6 \%$ during anoxia (Hochachka et al., 1996). Protein synthesis is well known to be sensitive to the availability of both ATP and amino acids. In this manner, the suppression of RNA translation appears to be a protective response to deal with stresses, such as anoxia, that disrupt cellular ATP availability. Several studies have explored the in vivo protein synthesis rates during anoxia-induced metabolic depression in turtles (Brooks and Storey, 1993; Land et al., 1993; Fraser et al., 2001). Fraser et al. (2001) stated that the rates of protein synthesis in several tissues of $T$. scripta elegans given $1 \mathrm{~h}$ anoxia exposure showed no significant changes from control values. However, these rates soon decreased to $\sim 0 \%$ (below measurable values) when the duration of anoxia was increased to $3 \mathrm{~h}$ at $23^{\circ} \mathrm{C}$. These results are comparable to those obtained from isolated T. scripta elegans hearts (Bailey and Driedzic, 1995; Bailey and Driedzic, 1996). Additionally, experiments using isolated hepatocytes from C. picta bellii, documented a reduction to only $8 \%$ of normoxic protein synthesis rates after $12 \mathrm{~h}$ of anoxia (Land et al., 1993).

By evaluation of translational rates after exposure to anoxia, it has been shown that $T$. scripta elegans successfully suppresses protein synthesis without the generation of a 'protein debt' (Fraser et al., 2001). Rates of translation were shown to be unchanged from normoxic values in $T$. scripta elegans tissues after $3 \mathrm{~h}$ recovery from $3 \mathrm{~h}$ of anoxic exposure (Fraser et al., 2001). Again, these results are comparable to those obtained from isolated T. scripta elegans hearts after $1 \mathrm{~h}$ recovery from $2 \mathrm{~h}$ of anoxia (Bailey and Driedzic, 1997). The inhibition of protein translation during hypometabolism might be achieved in three ways: (1) through a reduction of the amount of mRNA substrate 
available, (2) through differential regulation of the ribosomal translational machinery, and (3) via microRNA interference.

Due to the high cost of transcription (about 10\% of ATP turnover during normoxia) one would expect to see a downregulation of RNA synthesis under anoxia, leading to the reduction in protein synthesis. However, neither total mRNA content nor the mRNA transcript levels of most constitutively expressed genes were actually reduced under anoxic conditions in the anoxic turtles (Douglas et al., 1994). For example, complementary DNA (cDNA) array screening of liver tissue of T. scripta elegans (control vs. $5 \mathrm{~h}$ anoxia) revealed that $93-95 \%$ of the genes examined showed no change in transcript levels. A putative up-regulation of 3\% and a down-regulation of $4 \%$ of genes were seen for T. scripta elegans liver after $5 \mathrm{~h}$ anoxia. Similarly, other studies have shown that the RNA-to-protein ratio does not significantly change in T. scripta elegans liver after $12 \mathrm{~h}$ of anoxia (Land et al., 1993). Additionally, complementary studies have documented no change in total translatable RNA concentrations after $16 \mathrm{~h}$ of anoxia or recovery in the liver, kidney, heart and red and white skeletal muscle of T. scripta elegans (Douglas et al., 1994). Hence, the decrease in protein synthesis rates exhibited during anoxia does not appear to be controlled by tissue RNA concentration. Instead, reversible control of the rate of protein synthesis during anoxia-induced metabolic rate depression is likely at play in the anoxic turtle, a process that could be mediated by either regulation of translational complexes or non-coding RNA (microRNA) control.

In addition to global translational regulation by overall RNA abundance, inhibition of the translation of specific mRNA transcripts can also be accomplished via differential regulation of targeting microRNAs. MicroRNAs (miRNAs) are short, non- 
coding RNAs capable of regulating protein expression within a cell (Figure 1.5). These 18-25 nucleotide transcripts are able to bind with full or partial complementarity usually to the 3' untranslated regions (UTR) of mRNA targets, resulting in the inhibition of translation or degradation of that target (Leung and Sharp, 2010; Grimson et al., 2007; Schier and Giraldez, 2006). It is estimated that at least $60 \%$ and up to $90 \%$ of all mammalian mRNAs may be targeted by miRNAs (Friedman and Avraham, 2009; Perron and Provost, 2008), and at this time over 1000 miRNAs have been identified in the human genome. A single miRNA may target multiple mRNAs, and a single mRNA may have multiple miRNA binding sites (Lewis et al., 2003; Bartel, 2004). Simply due to their sequence diversity and the fact that they are predicted to target the majority of mRNAs in mammals (and likely in all animals), this regulatory pathway is of great importance. In fact, through a myriad of comparative expression analyses and gain- and loss-of function experiments, miRNAs have been shown to be critically involved in regulating the expression of proteins involved in biological development, cell differentiation, apoptosis, cell cycle control, stress response and disease pathogenesis (Leung and Sharp, 2010; Shi and Jin, 2010; Chan et al., 2005; Ivey and Srivastava, 2010). Given the roles of miRNAs in a wide variety of cellular processes, it would seem likely that these non-coding RNAs could play critical roles in regulating protein translation in anoxic turtles. For example, the activity of signaling networks can be susceptible to changes in protein abundance. The ability of microRNAs to influence protein amount could yield significant control of the regulation of these signalling networks, effectively changing the signalling landscape and reprioritizing ATP metabolism during periods of stress (Cui et al., 2006; Inui et al., 2010). 
Furthermore, a recent study examining the effect of miRNA on the synthesis of several thousand proteins (achieved via miRNA transfection or miRNA knockdown) showed that changes in a single miRNA can directly decrease the production of hundreds of proteins (Selbach et al., 2008). It would therefore be expected that even moderate changes in microRNA expression would yield widespread effects similar to those seen in studies documenting translational repression during hypometabolism, facilitated by a combination of mRNA repression and degradation (Fraser et al., 2001; Fuery et al., 1998; Smith et al., 1999; Guppy et al., 2000; Larade and Storey, 2002; Guo et al., 2010). Given the nature of miRNA targeting and the translational impact of a single miRNA, small changes in miRNA expression could facilitate rapid translational repression (11-16\%) and/or degradation (84-89\%) of numerous target genes (Guo et al., 2010). The extent to which mRNA-miRNA pairing occurs may allow the expression of key genes necessary for survival. For example, mRNA-miRNA interactions may allow for the translation of HSPs necessary for protein folding, while inhibiting expression of proteins involved in cellular proliferation.

Highlighting the role of miRNAs in the regulation of hypometabolism in tolerant animal models, studies are now finding that microRNA expression patterns show stressdependent differences in hibernating mammals (ground squirrels, little brown bats), and freeze tolerant animals (wood frogs, periwinkles, hatchling painted turtles) (Morin et al., 2008; Biggar et al., 2009; Biggar and Storey, 2011; Biggar et al., 2012a; Biggar et al., 2012b; Shaffer et al., 2013). A study examining the expression of miRNA during freeze tolerance in wood frogs found differential expression of $m i R-16-1$ in liver; this key microRNA that plays a role in arresting the cell cycle (Biggar et al., 2009). Since it is of 
critical importance to reduce ATP-costly processes during hypometabolism, miRNAs may aid in the reprioritization of ATP use. Although miRNA research has only begun in anoxic T. scripta elegans, existing research from other animals that use reversible metabolic depression provides an indication that miRNAs may play a role in achieving a hypometabolic state among stress-tolerant turtles.

\section{Hypothesis}

The biochemical mechanisms of anoxia tolerance in the freshwater turtle (Trachemys scripta elegans) involve the reversible arrest of the cell cycle and changes to selected master proteins, enzymes, and microRNAs that mediate the repression of necessary proteins involved in cell proliferation to elicit control at both the transcriptional and translation level.

To address this hypothesis, I investigated specific regulatory pathways, proteins and microRNAs listed in the following objectives:

\section{Objective 1: Profiling the State of the Cell Cycle during Anoxia}

Although it is clear that anoxic survival relies on the suppression of ATP consuming processes, the state of the cell cycle in anoxia tolerant organisms remains unknown. Proliferation (or replacement) of cells are clearly energy-expensive biosynthetic processes, so it would make sense that these activities are suppressed to a minimum under anoxic conditions. The demanding energetics of mitosis provides an intriguing suggestion that the cell cycle may arrest in proliferating tissues in order to 
facilitate metabolic rate depression and play an important role in cellular energyconserving responses to environmental stresses.

Specific Hypothesis 1: Key proteins involved in the regulation of cellular proliferation are regulated such that the cell cycle will slow-down or arrest during when oxygen is limiting, supporting ATP conservation and anoxia survival in T. scripta elegans.

Chapter 2 tests this hypothesis by exploring the regulation of Cyclin and Cdk proteins and mRNA in turtle organs in response to anoxia. The aim of this chapter is to create a general profile of the state of the cell cycle during anoxia in both proliferative (liver and kidney) and post-mitotic (white skeletal muscle) tissues. It is important to learn if, and at what stage, the cell cycle may be regulated under anoxic conditions in tolerant species and whether this regulation is accomplished through an evolutionarily conserved mechanism of proliferation quiescence. Specifically, this objective was partially addressed in this chapter by characterizing the protein and mRNA expression of Cyclins (type D1, E1, A and B) and Cdks (type 4, 6, 2 and Cdc2), key regulators of the cell cycle. Reduced levels of either Cyclins or Cdks could indicate whether cellular proliferation becomes synchronized, slows down or arrests during anoxia.

Chapter 3 further tests the hypothesis by examining the regulation of Cdk inhibitors in a phase-specific manner. Specifically, the chapter focuses on the regulation of Cdk proteins characterizing the expression of both Cdk activators (Cdc25a and $\mathrm{Cdc} 25 \mathrm{c})$ and inhibitors (p16, p27 and p21) in response to anoxia. Regulation in the $\mathrm{G}_{1}$ 
phase of the cell cycle typically involves the Cdk inhibitors p16 and p21 as well as the Cdk activator, Cdc25a. However, regulation in either the $G_{2}$ or $G_{1}$ phases of the cell cycle involves the Cdk inhibitor p21 and the activator $\mathrm{Cdc} 25 \mathrm{c}\left(\mathrm{G}_{2}\right.$-specific). Hence, an analysis of phase-specific inhibitors and activators will yield critical information as to which phases of the cell cycle are highly regulated during anoxia.

Chapter 4 tests the hypothesis by exploring the regulation of specific, DNAbound, mechanisms of $\mathrm{G}_{0} / \mathrm{G}_{1}$ phase arrest of the cell cycle. This chapter also addresses this objective by exploring both the differential regulation and protein interactions that are markers of either $G_{0}$ or $G_{1}$ cell cycle arrest. Specially, the study analyzed the protein make-up of inhibitory transcriptional complexes built upon E2F family members as this is the transcriptional complex that directs the cell cycle to arrest in the $\mathrm{G}_{1}$ phase, or enter reversible quiescence $\left(\mathrm{G}_{0}\right)$. This chapter required the creation and optimization of an experiment developed specifically to measure the relative presence of proteins bound to DNA-bound E2F protein in order to determine whether $\mathrm{G}_{1}$ or $\mathrm{G}_{0}$-specific DNA-bound complexes formed under anoxic conditions in the turtle.

\section{Objective 2: Activation of signaling pathways involved in Cell Cycle regulation}

Several signaling pathways are able to either arrest or activate the cell cycle in response to different cellular stresses (UV damage, oxidative stress and nutrient restriction). Studies have shown that the Akt signaling pathway can positively respond to favorable growth conditions and promote the expression of Cyclin D1 protein, initiating cellular proliferation. Conversely, the ATM/ATR signaling pathway responds to periods of environmental stress and arrests the cell cycle until favorable conditions are restored. 
Interestingly, little is known about the regulation of these signaling pathways or their influence on the cell cycle in response to anoxia in T. scripta elegans.

Specific Hypothesis 2: Anoxic survival by T. scripta elegans includes the inactivation of the Akt pathway that promotes cell cycle activity and the activation of the ATM/ATR cell cycle arrest pathway.

Chapter 2 tests this hypothesis by examining the response of Akt to periods of anoxia and its indirect effects on the initiation of the cell cycle. The initiation of cell proliferation is driven by the presence of extrinsic mitogens acting upon the PI3K/Akt pathway to prevent the degradation of Cyclin D1 through the regulation of GSK-3 $\beta$. This chapter explores the phosphorylation status (activation) of Akt, the phosphorylation status (inactivation) of its downstream target, GSK-3 $\beta$ and the phosphorylation status (inactivation) of the crucial downstream target of GSK-3 $\beta$, Cyclin D1. The ultimate effect of Akt signaling on the cell cycle is the inactivation of GSK-3b, preventing the phosphorylation and subsequence degradation of Cyclin D1 protein.

Chapter 3 tests this hypothesis by examining the relative phosphorylation (and activation) of downstream targets of ATM and ATR, namely Chk1 and Chk2. Both Chk1 and Chk2 are regulated and activated via phosphorylation by these two stress response protein kinases that are known to be triggered by periods of cellular stress. The downstream targets of both Chk1 and Chk2 that are effectors of the cell cycle will also studied, connecting the signaling by ATM and ATR to the downstream effects on the cell cycle. 


\section{Objective 3: Post-Transcriptional Regulation of the Cell Cycle by microRNA}

MicroRNAs are short, non-coding RNAs capable of regulating protein expression. These 18-25 nucleotide transcripts are able to bind with full or partial complementarity to mRNA targets, resulting in either translational inhibition or degradation of that target. The degree and extent of microRNA binding creates a complex regulatory system that has been predicted to be involved in almost every aspect of biological function. As such, the characteristics of microRNA regulation match the needs of metabolic depression to be broadly applicable, readily coordinated, easily induced and readily reversed, suggestive of its importance in establishing the hypometabolic state. Interestingly, microRNAs may provide a mechanism to regulate the cell cycle that is broadly applicable and can be easily induced and reversed such that few changes need to be made to protein composition during transitions to and from the stressed state.

Regulation by microRNA may also allow for rapid release of mRNA targets upon exit from anoxia, allowing rapid translation of proteins necessary for cell cycle initiation.

Hypothesis 3: MicroRNAs are involved in regulating the expression of key cell cycle-related proteins, resulting in a decrease in cellular proliferation under anoxic conditions in turtles.

Chapter 5 evaluates the responses of selected microRNAs to anoxia stress and their influence on cell cycle regulators. The expression of microRNAs known to regulate the cell cycle are analyzed as well as their binding potential to turtle mRNA targets, and correlation with target protein expression. Primary analysis examines the microRNA 
regulation of Cyclin D1, a protein whose expression is well-established to be regulated by microRNA and has known implications to cell cycle arrest. This chapter required the creation and optimization of an experiment developed specifically to amplify and sequence mature microRNA transcripts. 


\section{References}

Bailey, J.R., and Driedzic, W.R. 1995. Short-term anoxia does not impair protein turnover in isolated perfused turtle heart. J. Comp. Physiol. B, 164, 622-628.

Bailey, J.R., and Driedzic, W.R. 1996. Decreased total ventricular and mitochondrial protein synthesis during extended anoxia in turtle heart. Am. J. Physiol., 271, R1660-R1667.

Bailey, J.R., and Driedzic, W.R. 1997. Protein synthesis under conditions of anoxia and changing workload in ventricle strips from turtle heart. J. Exp. Zool., 278, 273-282.

Bartel, D. 2004. MicroRNAs: genomics, biogenesis, mechanism, and function. Cell, 116, 281-297.

Biggar, K., Dubuc, A. and Storey, K.B. 2009. MicroRNA regulation below zero: Differential expression of miRNA-21 and miRNA-16 during freezing in wood frogs. Cryobiology, 59, 317-321.

Biggar, K.K., and Storey, K.B. 2011. The emerging roles of microRNAs in the molecular responses of metabolic rate depression. J. Mol. Cell Biol., 3, 167-175.

Biggar, K.K., and Storey, K.B. 2012. Evidence for cell cycle suppression and microRNA regulation of cyclin D1 during anoxia exposure in turtles. Cell Cycle, 11, 17051713.

Biggar, K.K., Kornfeld, S.F. and Storey, K.B. 2012a. Differential expression of mature microRNAs involved in muscle maintenance of hibernating little brown bats, Myotis lucifugus: a model of muscle atrophy resistance. Genom. Proteom. Bioinform., 10, 295-301.

Biggar, K.K., Kornfeld, S.F., Maistrovski, Y. and Storey, K.B. 2012b. MicroRNA regulation in extreme environments: Differential expression of microRNAs in the intertidal snail Littorina littorea during extended periods of freezing and anoxia. Genom. Proteom. Bioinform., 10, 302-309.

Blokhina, O., Virolainen, E., and Fagerstedt, K.V. 2003. Antioxidants, oxidative damage and oxygen deprivation stress: a review. Ann. Bot., 91, 179-194.

Brierley, J.B. 1977. Experimental hypoxic brain damage. J. Clin. Path., 11, 181-187.

Brooks, S.P.J., and Storey, K.B. 1989. Regulation of glycolytic enzymes during anoxia in the turtle Pseudemys scripta. Am. J. Physiol., 257, R278-R283.

Brooks, S.P.J., and Storey, K.B. 1993. De novo protein synthesis and protein phosphorylation during anoxia and recovery in the red-eared turtle. Am. J. Physiol., 265, R1380-R1386.

Brooks, S.P.J., and Storey, K.B. 1995. Protein phosphorylation patterns during aestivation in the land snail Otala lactea. Mol. Cell. Biochem., 143, 7-13.

Chan, J., Krichevsky, A., and Kosik, K. 2005. MiRNA-21 is an antiapoptotic factor in human glioblastoma cells. Cancer Res., 65(14), 6029-6033. 
Clark, V.M., and Miller, A.T. 1973. Studies on anaerobic metabolism in the fresh-water turtle (Pseudemys scripta elegans). Comp. Biochem. Physiol. A, 44, 55-62

Cowan, K.J., and Storey, K.B. 2003. Mitogen-activated protein kinases: new signaling pathways functioning in cellular responses to environmental stress. J. Exp. Biol. 206, 1107-1115.

Cramer, F., Englisch, U., Freist, W., and Sternbach, H. 1991. Aminoacylation of tRNAs as critical step of protein biosynthesis. Biochimie, 73, 1027-1035.

Cui, Q., Yu, Z., Purisima, E.O., and Wang, E. 2006. Principles of microRNA regulation of a human cellular signaling network. Mol. Sys. Biol., 2(46), 1-7.

Dieni, C.A., and Storey, K.B. 2009. Creatine kinase regulation by reversible phosphorylation in frog muscle. Comp. Biochem. Physiol. B, 152, 405-412.

Doll, C.J., Hochachka, P.W., and Reiner, P.B. 1991. Reduced ionic conductance in the turtle brain. Am. J. Physiol. 261, R1321-R1324.

Douglas, D.N., Giband, M., Altosaar, I., and Storey, K.B. 1994. Anoxia induces changes in translatable mRNA populations in turtle organs: a possible adaptive strategy for anaerobiosis. J. Comp. Physiol. B, 164, 405-414.

Embley, M.T., and Martin, W. 2006. Eukaryotic evolution, changes and challenges. Nature, 440, 623-630.

Fraser, K.P., Houlihan, D.F., Lutz, P.L., Leone-Kabler. S., Manuel, L., and Brechin, J.G. 2001. Complete suppression of protein synthesis during anoxia with no post-anoxia protein synthesis debt in the red-eared slider turtle Trachemys scripta elegans. J. Exp. Biol., 204, 4353-4360.

Friedman, L., and Avraham, K. 2009. MicroRNAs and epigenetic regulation in the mammalian_inner ear: implications for deafness. Mamm. Genome, 20, 581-603.

Fuery, C.J., Withers, P.C., Hobbs, A.A., and Guppy, M. 1998. The role of protein synthesis during metabolic depression in the Australian desert frog, Neobatrachus centralis. Comp. Biochem. Phys. A, 119, 469-476.

Gatten, R.E. 1987. Cardiovascular and other physiological correlates of hibernation in aquatic and terrestrial turtles. Amer. Zool., 24, 59-68.

Gordos, M., and Franklin, C.E. 2002. Diving behaviour of two Australian bimodally respiring turtles, Rheodytes leukops and Emydura macquarii, in a natural setting. J. Zool., 258(3), 335-342.

Greenway, S.C., and Storey, K.B. 2000. Mitogen-activated protein kinases and anoxia tolerance in turtles. J. Exp. Zool. 287, 477-484.

Grimson, A., Farh, K., Johnston, W., Garrett-Engele, P., and Bartel, D. 2007. MicroRNA targeting specificity in mammals: determinants beyond seed pairing. Mol. Cell, 27, 91-105.

Guo, H., Ingolia, N.T., Weissman, J.S., and Bartel, D.P. 2010. Mammalian microRNAs predominantly act to decrease target mRNA levels. Nature, 466, 835-841. 
Guppy, M., Reeves, D.C., Bishop, T., Withers, P., Buckingham, J.A., and Brand, M.D. 2000. Metabolic depression in animals: physiological perspectives and biochemical generalizations. FASEB J., 14, 999-1004.

Hajnoczky, G., Davies, E., and Madesh, M. 2003. Calcium signaling and apoptosis. Biochem. Biophys. Res. Commun., 304(3), 445-454.

Herbert, C.V., and Jackson, D.C. 1985. Temperature effects on the responses to prolonged submergence in the turtle Chrysemys picta bellii. I. Blood acid-base and ionic changes during and following anoxic submergence. Physiol. Zool., 58, 655669.

Hochachka, P.W. 1988. Metabolic suppression and oxygen availability. Can. J. Zool. 66, 152-158.

Hochachka, P.W., Buck, L.T., Doll, C.J., and Land, S.C. 1996. Unifying theory of hypoxia tolerance: Molecular/metabolic defense and rescue mechanisms for surviving oxygen lack. Proc. Natl. Acad. Sci. USA, 93(18), 9493-9498.

Hochachka, P.W., Land, S.C., and Buck, L.T. 1997. Oxygen sensing and signal transduction in metabolic defense against hypoxia: lessons from vertebrate facultative anaerobes. Comp. Biochem. Physiol. A, 118(1), 23-29.

Hochachka, P.W., and Lutz P.L. 2001. Mechanism, origin and evolution of anoxia tolerance in animals. Comp. Biochem. Physiol. B, 130, 435-459.

Hoeflich, K.P., and Woodgett, J. R. 2001. Mitogen-activated protein kinases and stress. in Cell and Molecular Responses to Stress, Vol. 2, K.B. Storey and J.M. Storey (Eds.), Elsevier Press, Amsterdam, pp. 175-193.

Inui, M., Martello, G., and Piccolo, S. 2010. MicroRNA control of signal transduction. Nat. Rev. Mol. Cell Biol., 11, 252-263.

Ivey, K., and Srivastava, D. 2010. MicroRNAs as regulators of differentiation and_cell_fate decisions. Cell. Stem Cell, 7(1), 36-41.

Jackson, D.C. 1968. Metabolic depression and oxygen depletion in the diving turtle. J. Appl. Physiol., 24(4), 503-9.

Jackson, D.C. 1997. Lactate accumulation in the shell of the turtle, Chrysemys picta bellii, during anoxia at 3 and $10^{\circ}$ C. J. Exp. Biol., 200(7), 2295-2300.

Jackson, D.C. 2002. Hibernating without oxygen: physiological adaptations of the painted turtle. J. Physiol., 543, 731-737.

Jackson, D.C. 2011. Life in a Shell: a Physiologist's View of a Turtle. Harvard University Press, Boston.

Jackson, D.C., Crocker, C.E., and Ultsch, G.R. 2000. Bone and shell contribution to lactic acid buffering of submerged turtles Chrysemys picta bellii at $3^{\circ} \mathrm{C}$. Am. J. Physiol. Regul. Integr. Comp. Physiol., 278, R1564-R1571.

Jackson, D.C., Taylor, S.E., Asare, V.S., Villarnovo, D., Gall, J.M., and Reese, S.A. 2007. Comparative shell buffering properties correlate with anoxia tolerance in freshwater turtles. Am. J. Physiol. Regu. Physiol., 292(2), R1008-R1015. 
Johnson, G.L., and Lapadat, R. 2002. Mitogen-activated protein kinase pathways mediated by ERK, JNK, and p38 protein kinases. Science, 298(5600), 1911-1912.

Kelly, D.A., and Storey, K.B. 1988. Organ-specific control of glycolysis in the anoxic turtle. Am. J. Physiol. Regu. Physiol., 225(5), R774-R779.

Land, S.C., Buck, L.T., and Hochachka, P. W. 1993. Response of protein synthesis to anoxia and recovery in anoxia-tolerant hepatocytes. Am. J. Physiol., 265, R41-R48.

Land, S.C., and Hochachka, P.W. 1994. Protein turnover during metabolic arrest in turtle hepatocytes: role and energy dependence of proteolysis. Am. J. Physiol. Cell. Physiol., 266(4), C1028-C1036.

Larade, K., and Storey, K.B. 2002. A profile of the metabolic responses to anoxia in marine invertebrates. Cell Mol. Resp. Stress, 3, 27-46.

Leung, K., and Sharp, P. 2010. MicroRNA functions in stress responses. Mol. Cell, 40, 205-215.

Lewis, B., Shih, I., Jones-Rhoades, M., Bartel, D., and Burge, C. 2003. Prediction of mammalian microRNA targets. Cell, 115, 787-798.

Lutz, P.L., McMahon, P., Rosenthal, M., and Sick, T.J. 1984. Relationships between_aerobic and anaerobic energy production in turtle brain in situ. Am. J. Physiol., 247(16), R740-R744.

Lutz, P.L., and Storey, K.B. 1997. Adaptations to variations in oxygen tension by vertebrates and invertebrates. Handbook of Physiology, Section 13, Comparative Physiology, W. H. Dantzler (Ed.), Oxford University Press, Oxford, 1479-1522.

Lutz, P.L., and Milton, S.L. 2004. Negotiating brain survival in the turtle. J. Exp. Biol., 207, 3141-3147.

MacDonald, J.A., and Storey, K.B. 1999. Regulation of ground squirrel $\mathrm{Na}^{+} \mathrm{K}^{+}$-ATPase activity by reversible phosphorylation during hibernation. Biochem. Biophys. Res. Commun., 254(2), 424-429.

Marais, E., Genade, S., Huisamen, B., Strijdom, J. G., Moolman, J. A., and Lochner, A. 2001. Activation of p38 MAPK Induced by a multi-cycle sschaemic preconditioning protocol is associated with attenuated p38 MAPK activity during sustained ischaemia and reperfusion. J. Mol. Cell. Cardiol., 33, 769-778.

Morin, P., Dubuc, A. and Storey, K.B. 2008. Differential expression of microRNA species in organs of hibernating ground squirrels: a role in translational suppression during torpor. Biochim. Biophys. Acta 1779, 628-633.

Nilsson, G.E., and Renshaw, G.M. 2004. Hypoxic survival strategies in two fishes: extreme anoxia tolerance in the North European crucian carp and natural hypoxic preconditioning in a coral-reef shark. J. Exp. Biol., 207(18), 3131-3139. 
Orrenius, S., Zhivotovsky, B., and Nicotera, P. 2003. Regulation of cell death: the calcium-apoptosis link. Nature Rev. Mol. Cell Biol., 4, 552-565.

Park, K. M., Chen, A., and Bonventre, J. V. 2001. Prevention of kidney ischemia/reperfusion-induced functional injury and JNK, p38, and MAPK kinase activation by remote ischemic pretreatment. J. Biol. Chem., 276,11870-11876.

Perron, M., and Provost, P. 2008. Protein interactions and complexes in human microRNA biogenesis and function. Front. Biosci., 13, 2537-2547.

Reese, S.A., Jackson, D.C., and Ultsch, G.R. 2003. Hibernation in freshwater turtles: softshell turtles (Apalone spinifera) are the most intolerant of anoxia among North American species. J. Comp. Physiol. B, 173(3), 263-268.

Schier, A., and Giraldez, A. 2006. MicroRNA function and mechanism: insights from zebra fish. Cold Spring Harbour Symposia on Quantitative Biology, 121, 195-203.

Selbach, M., Schwanhausser, B., Thierfelder, N., Fang, Z., Khanin, R., and Rajewsky, N. 2008. Widespread changes in protein synthesis induced by microRNA. Nature, 455 , $58-63$.

Shaffer, H.B., Minx, P., Warren, D.E., Shedlock, A.M., Thomson, R.C., Valenzuela, N., Abramyan, J., Badenhorst, D., Biggar, K.K., Borchert, G.M., Botka, C.W., Bowden, R.M., Braun, E.L., Bronikowski, A.M., Bruneau, B.G., Buck, L.T., Capel, B., Castoe, T.A., Czerwinski, M., Delehaunty, K.D., Edwards, S.W., Fronick, C.C., Fujita, M.K., Fulton, L., Graves, T.A., Green, R.E., Haerty, W., Hariharan, R., Hillier, L.W., Holloway, A.K., Janes, D., Janzen, F.J., Kandoth, C., Kong, L., de Koning, A.P.J., Li, Y., Literman, R., McGaugh, S.E., Mork, L., O’Laughlin, M., Paitz, R.T., Pollock, D.D., Ponting, C.P., Radhakrishnan, S., Raney, B.J., Richman, J.M., St. John, J., Schwartz, T., Sethuraman, A., Spinks, P.Q., Storey, K.B., Thane, N., Vinar, T., Zimmerman, L.M., Warren, W.C., Mardis, E.R. and Wilson, R.K. 2013. The painted turtle genome: the evolution of extreme physiological adaptations in a slowly evolving lineage. Genome Biol., 14, R28.

Shi, Y., and Jin, Y. 2009. MicroRNA in cell differentiation and development. Sci. China C Life Sci., 52(3), 205-211.

Smith, R.W., Houlihan, D.F., Nilsson, G.E., and Brechin, J.G. 1999. Tissue-specific changes in protein synthesis rates in vivo during anoxia in crucian carp. Am. J. Physiol., 271, R897-R904.

Storey, K.B., and Storey, J.M. 1990. Metabolic rate depression and biochemical adaptation in anaerobiosis, hibernation and estivation. Q. Rev. Biol., 65(2), 145 174.

Storey, K.B. 2004. Molecular mechanisms of anoxia tolerance. Int. Cong. Ser., 1275, 4754.

Storey, K.B. and Storey, J.M. 2004a. Oxygen limitation and metabolic rate depression. In: Functional Metabolism: Regulation and Adaptation (Storey, K.B., ed.) WileyLiss, Hoboken, NJ, pp. 415-442. 
Storey, K.B., and Storey, J.M. 2004b. Metabolic rate depression in animals: transcriptional and translational controls. Biol. Rev. Camb. Philos. Soc., 79, 207233.

Storey, K.B. 2007. Anoxia tolerance in turtles: metabolic regulation and gene expression. Comp. Biochem. Physiol. A., 147, 263-276.

Storey, K.B., and Storey, J.M. 2007. Tribute to P.L. Lutz: Putting life on 'pause' molecular regulation of hypometabolism. J. Exp. Biol., 210, 1700-1714.

Storey, K.B., and Storey, J.M. 2010. Metabolic regulation and gene expression during aestivation. In: Aestivation: Molecular and Physiological Aspects (Navas, C.A. and Carvalho, J.E., eds), Progress in Molecular and Subcellular Biology, Springer, Heidelberg, Vol. 49, pp. 25-45.

Tribble, D.L., Aw, T.Y., and Jones, D.P. 1987. The pathophysiological significance of lipid peroxidation in oxidative cell injury. Hepatology, 7(2), 337-386.

Ultsch, G. 1985. The viability of nearctic freshwater turtles submerged in anoxia and normoxia at 3 and 10 degrees C. Comp. Biochem. Physiol. A, 81, 607-611.

Ultsch, G.R., and Jackson, D.C. 1982. Long-term submergence at 3C of the turtle, Chrysemys picta bellii, in normoxic and severely hypoxic water. I. survival, gas exchange and acid-base status. J. Exp. Biol. 96, 11-28.

Ultsch, G.R., and Jackson, D.C. 1987. Long-term submergence at 3C of the turtle, Chrysemys picta bellii, in normoxic and severely hypoxic water. III. Effects of changes in ambient PO2 and subsequent air breathing. J. Exp. Biol. 97, 87-99.

Willmore, W.G., and Storey, K.B. 1997. Antioxidant systems and anoxia tolerance in a freshwater turtle Trachemys scripta elegans. Mol. Cell. Biochem., 170, 177-85. 
A)

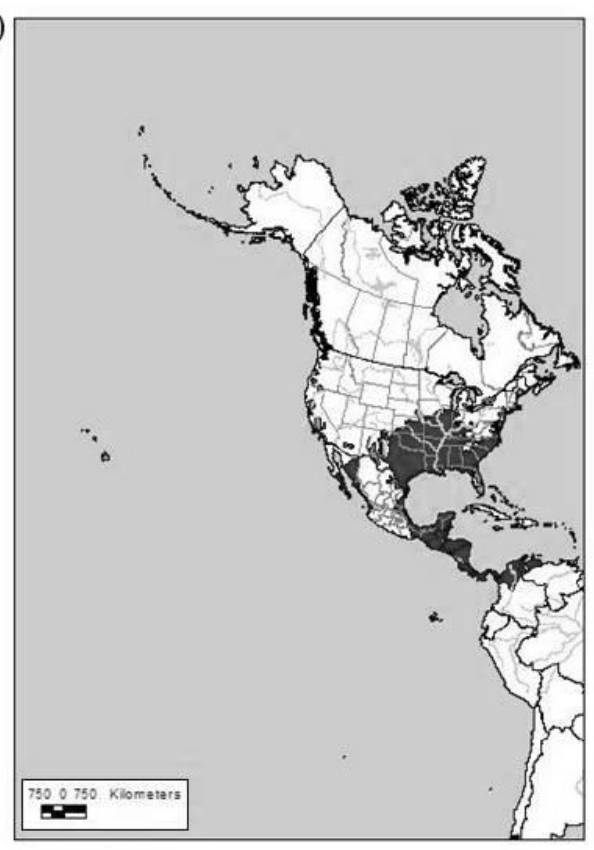

B)

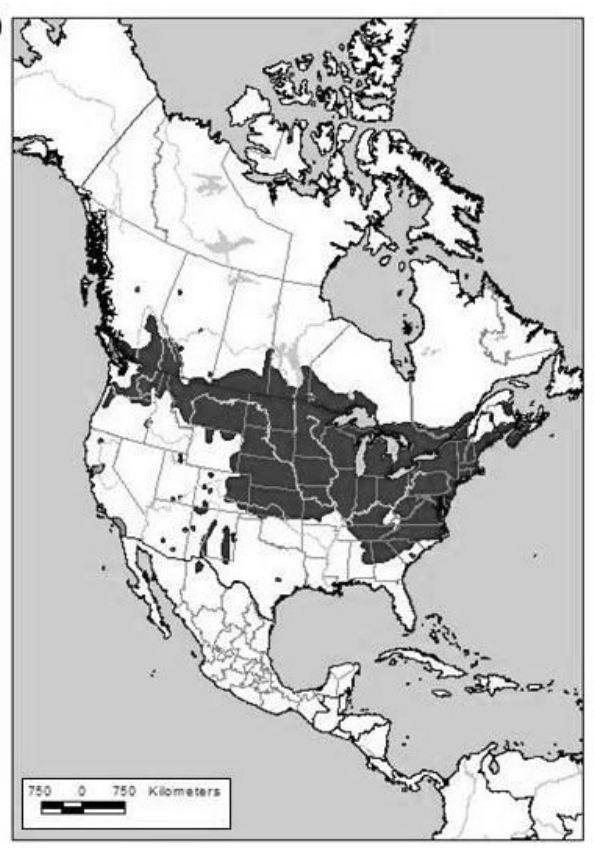

Figure 1.1 North American distribution of anoxia tolerant turtles, (A) Trachemys scripta elegans and (B) Chysemys picta. Shaded regions indicate populated regions of permanent residence. Distribution maps supplied from the Montana field guide. 


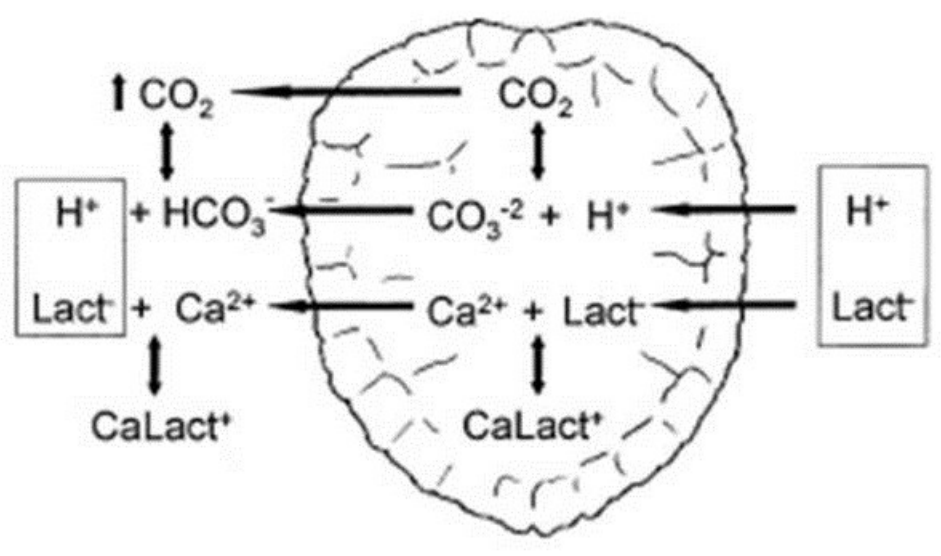

Figure 1.2 Lactate movement into a calcium carbonate rich shell during anoxia in the hypometabolic turtle. Shuttling of lactate to the shell during anoxia acts to buffer the acidic influence of lactic acid on intra- and extracellular $\mathrm{pH}$ levels. 


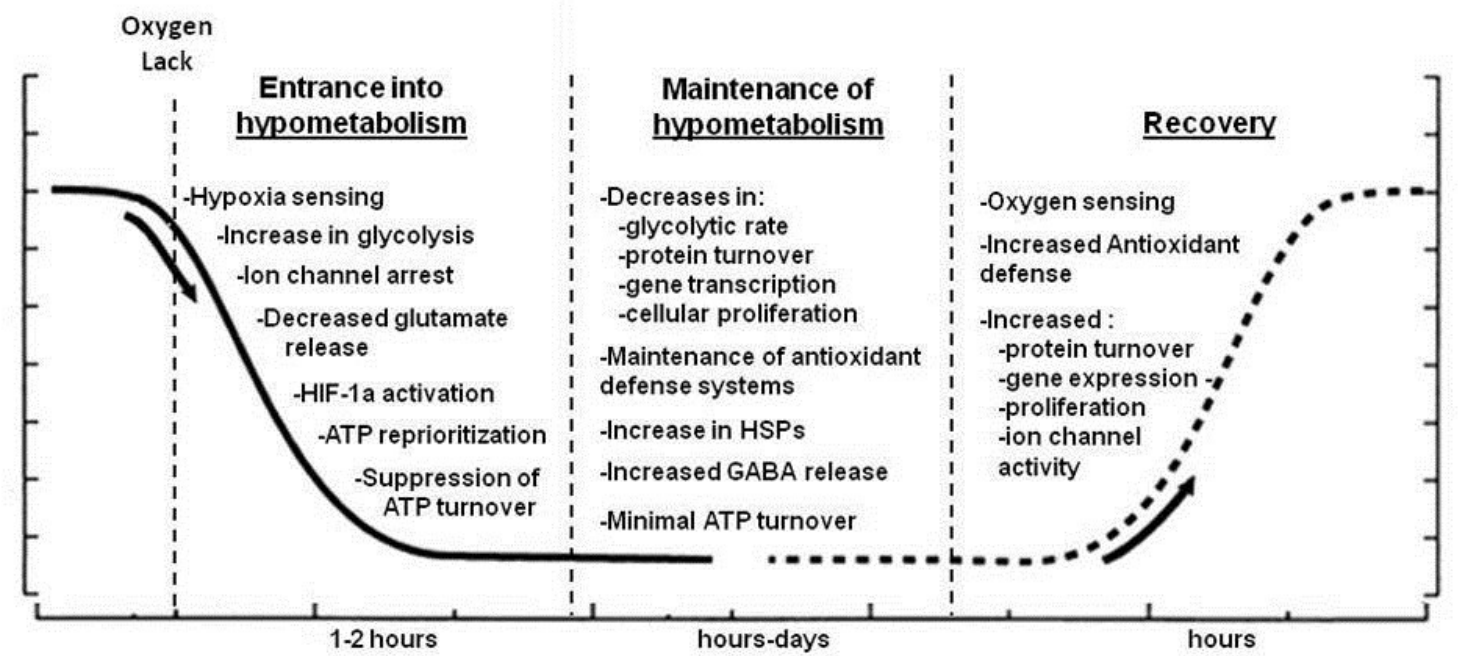

Figure 1.3 Transitions to and from a hypometabolic state in the anoxic turtle. Upon initial hypoxic sensing, cellular adjustments occur to reprioritize ATP metabolism and defend the cell from oxidative damage upon oxygen reperfusion. 


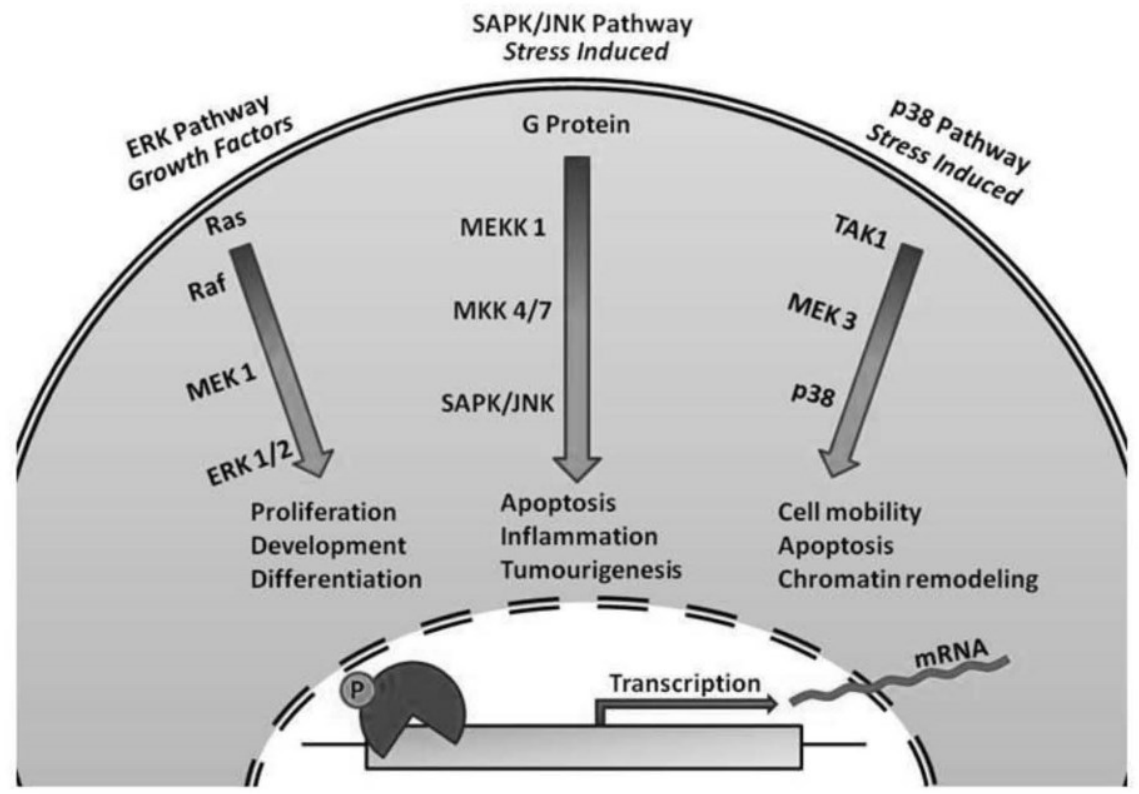

Figure 1.4 Generalized signaling pathways of ERK, SAPK/JNK, and p38 including their influences on cell functions. 


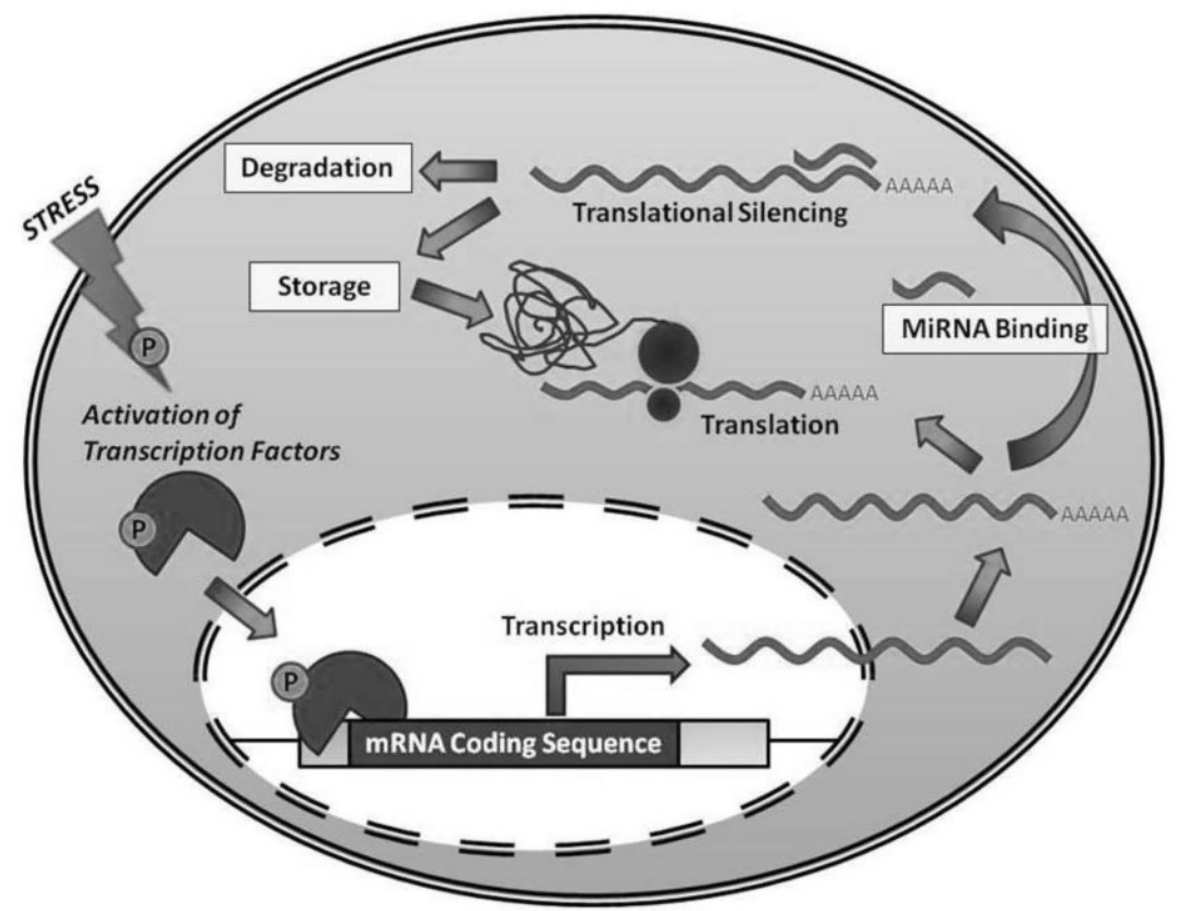

Figure 1.5 General mechanism of translational regulation by microRNA. MicroRNAs are targeted to the 3' UTR of specific mRNA transcripts. Depending on either perfect or imperfect base-pairing, miRNA:mRNA duplexes may targeted to degradation or temporary storage, respectively. 


\section{CHAPTER 2}

\section{PROFILE OF CELL CYCLE REGULATORY PROTEINS}




\subsection{Introduction}

The cell cycle consists of a series of events taking place up to and including cell division. The typical eukaryotic cell cycle consists of four distinct phases: $G_{1}$ phase, $S$ phase, $\mathrm{G}_{2}$ phase, and $\mathrm{M}$ phase (Figure 2.1), whereas cells that have reversibly stopped dividing are said to have entered a quiescence state, $G_{0}$. The first Gap phase $\left(G_{1}\right)$ functions to ensure that mechanisms are in place to control proper DNA synthesis. In some instances, the phase can be delayed to ensure proper DNA replication via the $G_{1}$ checkpoint (Johnson and Walker, 1999). The $\mathrm{G}_{1}$ phase is followed by the synthesis phase (S) where complete DNA replication occurs. After successful DNA replication, a subsequent gap phases $\left(\mathrm{G}_{2}\right)$ ensures that proper DNA replication has occurred and that the cells have successfully prepared for division. This arrest occurs via the $G_{2}$ DNAdamage checkpoint before entering mitosis (M phase). After successful completion of cellular division, each daughter cell begins in the $\mathrm{G}_{1}$ phase of a new cycle. Each preparatory phase $\left(\mathrm{G}_{1}, \mathrm{~S}\right.$ and $\left.\mathrm{G}_{2}\right)$ of the cell cycle has a distinct set of specialized molecular processes that prepare the cell for the initiation of mitosis (Johnson and Walker, 1999; Weinberg, 1995).

\subsubsection{Cell cycle regulation by Akt signaling}

The initiation of cellular proliferation is driven from the presence of extrinsic signaling pathways, such as the PI3K/Akt pathway, which prevents the degradation of Cyclin D1. The prevention of Cyclin D1 degradation by the PI3K/Akt pathway is accomplished through the direct inactivation of glycogen synthase kinase $3 \beta$ (GSK-3 $\beta$ )

(Figure 2.2). Several recent studies have shown that Cyclin D1 is upregulated in response 
to growth factors and is facilitated by the involvement of the insulin response PI3K/Akt pathway (Takuwa et al., 1999; Fielenbach and Antebi, 2008). The increased expression of Cyclin D1 is critical to the initiation of cellular proliferation and subsequent progression through the cell cycle. The PI3K/Akt pathway is initiated through insulin and insulin-like growth factor (IGF) signaling at the cell membrane through the insulin receptor. The primary downstream action triggered by receptor binding is activation of Akt, also known as protein kinase B (Lant and Storey, 2010). Akt has numerous important downstream targets that are conserved from $C$. elegans to humans, and which can initiate survival responses during prolonged periods of environmental stress (Lant and Storey, 2010). The phosphorylation state of Akt is the key point of regulation, determining Akt activity (Thr308) or inactivity (Ser473). Once active, Akt is able to commence signal transduction and has targets affecting numerous cellular processes and pathways, including the promotion of cellular proliferation (Lant and Storey, 2010).

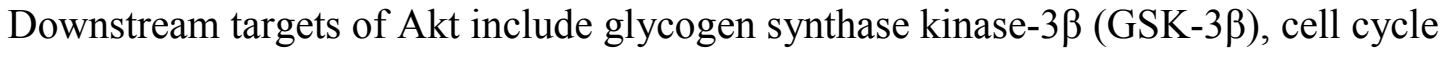
regulators (p21, p27) and regulators of protein synthesis (mTOR). The phosphorylation of GSK-3 $\beta$ (Ser9) by Akt inhibits its kinase function, thus preventing degradation of Cyclin D1 via GSK-3 $\beta$ dependent phosphorylation and thereby indirectly initiating the cell cycle. When active, GSK-3 $\beta$ is able to phosphorylate Cyclin D1 at amino acid Thr286 and targets Cyclin D1 for ubiquitination and subsequent proteolysis (Yang et al., 2006). 


\subsubsection{Cyclin and Cdk regulation of the cell cycle}

Initial activation of Cdk4 and Cdk6 leads to sequential phase-specific $\mathrm{Cdk}$ activation, creating a hierarchal system ensuring that one phase of the cell cycle is completed before the initiation of the next (Obaya and Sedivy, 2002) (see Figures 2.1 and 2.2). The ordered progression through cell-cycle phases is controlled by the sequential phosphorylation and activation of $\mathrm{Cdk} 4 \rightarrow \mathrm{Cdk} 6 \rightarrow \mathrm{Cdk} 2 \rightarrow \mathrm{Cdc} 2$ (also known as Cdk1) (Guardavaccaro and Pagano, 2006). Cdk activity is regulated by several mechanisms including the binding of a cyclin regulatory subunit and the association/disassociation of Cdk inhibitors (CKIs). Signaling begins the cycle through the PI3K/Akt pathway, acting to increase Cyclin D1 expression and facilitating the assembly with Cdk4 and Cdk6 to form pre-activated kinase complexes (Harrisingh et al., 2004). In contrast, Cyclin E1 is triggered by the internal E2F signaling pathway and the expression of Cyclin E1/Cdk2 complexes has been associated with the R-point transition (Figure 2.3). Remaining Cyclin/Cdk complexes trigger the activation of the next stages, and as a result, drive the cell cycle further through progression. During periods of cellular stress, the inhibition of Cyclin/Cdk complexes at the $\mathrm{G}_{1} / \mathrm{S}$ checkpoint leads to the disruption of the cycle and the downstream activation of Cyclin/Cdk complexes. The cyclic expression of Cdks and Cyclins in a specific phase of the cell cycle allows these proteins to be used as cell cycle markers that when compared can yield information regarding the current phase of synchronized cells (Guardavaccaro and Pagano, 2006).

The basic framework of the cell cycle consists of Cyclin/Cdk complex formation. The dependence of Cdk activity on Cyclin binding represents the primary mechanism whereby Cyclins mediate Cdk function by reconfiguring the alignment of residues 
involved in ATP binding and the repositioning of a 'T-loop' within the Cdk structure (Ortega et al., 2002). Beginning from quiescence, the introduction of growth factors allows for a depression of CKIs and an increased expression of Cyclin D1 (Obaya and Sedivy, 2002). Type D1 Cyclins are able to form active complexes with Cdk4 and Cdk6, functioning to initiate the cell cycle by facilitating the initial phosphorylation of Retinoblastoma (Rb) (Ser780) allowing partial activation of E2F1, a key cell cycle transcription factor (Boylan et al., 1999) (Figure 2.3). Since Cdk4 and Cdk6 proteins are expressed at relatively constant levels throughout the cell cycle, the expression of Cyclin $\mathrm{D} 1$ is the rate-limiting step in $\mathrm{Rb}$ phosphorylation, even during early $\mathrm{G}_{1}$ when $\mathrm{Cdk} 4$ and Cdk6 expression are highest (Matsushime et al., 1994).

Following activation of Cyclin D1/Cdk4 and Cyclin D1/Cdk6 complexes, and the partial phosphorylation of $\mathrm{Rb}$ (Ser780), Cyclin E1 is transcribed by E2F1 and its protein expression is enhanced, marking the entry into late $\mathrm{G}_{1}$ phase and the R-point transition (Lundberg and Weinberg, 1999) (Figure 2.3). Cyclin E1 forms an active kinase complex with Cdk2, leading to further phosphorylation and complete inactivation of Rb (Ser608), dissociating the inhibitory $\mathrm{Rb} / \mathrm{E} 2 \mathrm{~F}$ complex and allowing the expression of all genes required for S-phase entry and DNA replication (Harbour et al., 1999; Koff et al., 1992) (Figure 2.3). Soon after Cyclin E1 is expressed at the $\mathrm{G}_{1} / \mathrm{S}$ boundary, and cells commit to mitosis and begin to express Cyclin A1. Increased Cyclin A1/Cdk2 complexes facilitate the activation of DNA replication machinery and mark the S-phase. After completion of the S-phase and DNA replication, entry into $\mathrm{G}_{2}$ is marked by the expression of Cyclin B1, inactive cytoplasmic Cyclin B1/Cdc2 complexes, and the expression of active Cyclin $\mathrm{A} 1 / \mathrm{Cdc} 2$. Cyclin B1/Cdc2 remains inactivate late into the $\mathrm{G}_{2}$ phase, Cyclin $\mathrm{B} 1$ nuclear 
localization and Cdc2 activation being necessary for entry into mitosis (Maller, 1991; Nurse, 1990; Pines and Hunter, 1989). Targets of the Cyclin B1/Cdc2 complex include structural proteins involved in the execution and timing of mitotic events (Obaya and Sedivy, 2002; Long et al., 1998; Papst et al., 1998). In addition, Cyclins play an additional regulatory role through nuclear and cytoplasmic shuttling of Cdks, thereby suggesting a role where Cyclins act to regulate the cell cycle in a manner discrete from just-in-time translation (Ortega et al., 2002).

This chapter focuses on the biochemical adaptations that support survival of anoxia by the turtle, T. scripta elegans. In particular, the focus is on cell cycle suppression which could contribute to an overall reduction in energy consumption in the anoxic state. Arrest of cellular proliferation should be crucial for survival in the anoxic state, since aberrant activation of the cell cycle would lead to rapid depletion of energy stores. Data presented characterize the specific Cyclin and Cdk markers of each phase of the cell cycle and the signaling pathway known for its initiation. It is the aim of this chapter to create a general profile of the cell cycle during anoxia in proliferative (liver and kidney) and post-mitotic (white skeletal muscle) tissues. It is important to learn if, and at what stage, the cell cycle may be regulated during anoxia in tolerant species and whether this regulation is accomplished through an evolutionarily conserved mechanism of proliferative quiescence. 


\subsection{Materials and Methods}

\subsubsection{Animal Care and Treatment}

Adult female red-eared sliders (Trachemys scripta elegans), 700-1500 g, were acquired from local suppliers and held at $5 \pm 1{ }^{\circ} \mathrm{C}$ in large plastic tubs ( 2 turtles per tub) filled with dechlorinated tap water for several days before use. Control turtles were sampled from this condition. For anoxia exposure, turtles were transferred to large buckets at $5 \pm 1{ }^{\circ} \mathrm{C}$ that had been previously bubbled with $\mathrm{N}_{2}$ gas for $1 \mathrm{~h} ; 2-3$ turtles were added per bucket in 30 min intervals. Bubbling was continued for $1 \mathrm{~h}$ after the last turtle was added and was reinitiated again during sampling of the animals. A wire mesh was fitted into the tank about $5 \mathrm{~cm}$ below the water surface so that turtles remained submerged throughout the 5 or 20 hour experimental anoxia exposure. All animals were killed by decapitation and then tissues were rapidly dissected out, frozen in liquid nitrogen and stored at $-80^{\circ} \mathrm{C}$ until use. All animals were cared for in accordance with the guidelines of the Canadian Council on Animal Care and all experimental procedures had the prior approval of the Carleton University Animal Care Committee.

\subsubsection{Total and nuclear protein isolation}

For total protein isolation, samples of frozen tissues $(\sim 0.5 \mathrm{~g})$ were crushed under liquid nitrogen and then homogenized in $1.25 \mathrm{~mL}$ homogenizing buffer (20 mM Hepes pH 7.5, $200 \mathrm{mM} \mathrm{NaCl}, 0.1 \mathrm{mM}$ EDTA, $10 \mathrm{mM} \mathrm{NaF}, 1 \mathrm{mM} \mathrm{Na} \mathrm{VO}_{4}, 10 \mathrm{mM} \beta-$ glycerophosphate) with a few crystals of PMSF and $1 \mu \mathrm{L}$ Sigma protease inhibitor cocktail added (104 mM AEBSF, $80 \mu \mathrm{M}$ aprotinin, $4 \mathrm{mM}$ bestatin, $1.4 \mathrm{mM} \mathrm{E}-64,2 \mathrm{mM}$ leupeptin, $1.5 \mathrm{mM}$ pepstatin A) immediately before use. Samples were then centrifuged 
at $4{ }^{\circ} \mathrm{C}$ for $15 \mathrm{~min}$ at $10,000 \mathrm{xg}$ and the pellet was discarded. Soluble protein concentrations were assessed using the BioRad protein assay (Cat\# 500-0006) using bovine serum albumin as the standard. All samples were then adjusted to a constant concentration by adding a calculated small volume of homogenizing buffer. The samples were then mixed 1:1 v:v with 2 X SDS loading buffer (100 mM Tris-base, $4 \% \mathrm{w} / \mathrm{v}$ SDS, $20 \% \mathrm{v} / \mathrm{v}$ glycerol, $0.2 \% \mathrm{w} / \mathrm{v}$ bromophenol blue, $10 \% \mathrm{v} / \mathrm{v} 2$-mercaptoethanol). Final sample concentrations were $5 \mu \mathrm{g} / \mu \mathrm{L}$. Proteins were denatured by placing the tubes in boiling water for $5 \mathrm{~min}$. Samples were stored at $-40^{\circ} \mathrm{C}$ until use.

Nuclear extracts were prepared using a modification of the method above. Briefly, $\sim 0.5 \mathrm{~g}$ samples were homogenized using a Dounce homogenizer in $1 \mathrm{~mL}$ of homogenization buffer (10 mM Hepes pH 7.9, 10 mM KCl, 10 mM EDTA, 1 mM DTT). The DTT, as well as a few crystals of PMSF and $1 \mu \mathrm{L}$ of Sigma protease inhibitor cocktail were added just prior to homogenization. Samples were centrifuged at 10,000 $\times g$ for 10 min at $4{ }^{\circ} \mathrm{C}$ and the supernatant (cytoplasmic extract) was removed. The pellet was resuspended in $150 \mu \mathrm{L}$ of extraction buffer (20 mM HEPES, $400 \mathrm{mM} \mathrm{NaCl}, 1 \mathrm{mM}$ EDTA, $10 \% \mathrm{v} / \mathrm{v}$ glycerol, $1 \mathrm{mM}$ DTT). Again, the DTT, as well as $1 \mu \mathrm{L}$ of Sigma protease inhibitor cocktail, were added just prior to addition of the buffer to the pellet. Tubes containing the samples were put on ice horizontally on a rocking platform for $1 \mathrm{~h}$. Samples were then centrifuged at $10,000 \times g$ for 10 min at $4{ }^{\circ} \mathrm{C}$. The supernatant (nuclear extract) was collected. The extracts were quantified and treated as described above to create samples for western blotting. Final sample concentrations were $5 \mu \mathrm{g} / \mu \mathrm{L}$. The integrity of the nuclei was confirmed by immunoblotting of cytoplasmic and nuclear 
fractions and probing with histone $\mathrm{H} 3$ antibody (diluted v:v 1:1000; Cell Signaling; Cat\#9715).

\subsubsection{Immunoblotting}

Aliquots containing $20 \mu \mathrm{g}$ protein were loaded onto polyacrylamide gels, together with prestained molecular weight standards (FroggaBio; Cat\#PM005-0500) and separated using a discontinuous buffer system. Electrophoresis was carried out at $180 \mathrm{~V}$ for $45 \mathrm{~min}$ using the BioRad Mini-Protean 3 system with 1X Tris-glycine running buffer. Proteins on the gel were then electroblotted onto polyvinylidenedifluoride (PVDF) membrane (Millipore; Cat\#IPVH00010) using a BioRad mini Trans-Blot cell. The transfer was carried out at $160 \mathrm{~mA}$ constant amperage for $1.5 \mathrm{~h}$. Following the transfer, membranes were washed in TBST $(10 \mathrm{mM}$ Tris $\mathrm{pH} 7.5,150 \mathrm{mM} \mathrm{NaCl}, 0.05 \% \mathrm{v} / \mathrm{v}$ Tween-20) three times for $5 \mathrm{~min}$ each. The membranes were blocked using $1 \mathrm{mg} / \mathrm{ml}$ polyvinyl alcohol (PVA) (70-100 kDa) in TBST for 30-45 sec. After blocking, the membranes were probed with an antigen-specific antibody (see Appendix C). Experimental conditions that are specific to each antibody are listed in Appendix D. All membrane washes (after both primary and secondary antibody probing) consisted of three washes for 5 min each in TBST at room temperature. Subsequently, blots were developed using enhanced chemiluminescence.

Bands were visualized using a ChemiGenius bio-imaging system and band densities were quantified using the associated GeneTools software (Syngene, Frederick, MD). The protein molecular weight of the immunoreactive band band was confirmed as being the expected size by running separate lanes containing $3 \mu \mathrm{L}$ of protein ladder and a 
mammalian sample (control liver from thirteen-lined ground squirrel) as a positive control. Immunoblotting with $\alpha$-tubulin-specific antibodies showed constant $\alpha$-tubulin expression in all experimental conditions and when compared with the combined density of a group of Coomassie stained protein bands. Immunoblots of the proteins of interest were individually adjusted for loading irregularities by standardizing the band intensity of immune-reactive material in each lane against the combined density of a group of Coomassie-stained protein bands in the same lane. These Coomassie-stained proteins showed similar expression pattern to $\alpha$-tubulin and the same stained bands were used for each such comparison.

\subsubsection{Total RNA isolation}

Total RNA was isolated from liver, kidney and white skeletal muscle of control and anoxic (5 and 20 h) turtles using Trizol ${ }^{\mathrm{TM}}$ (Invitrogen; Cat\#15596-018). Briefly, 100 mg of tissue was homogenized in $1 \mathrm{~mL}$ Trizol using a Polytron homogenizer followed by the addition of $200 \mu \mathrm{L}$ of chloroform and centrifugation at $10,000 \mathrm{xg}$ for $15 \mathrm{~min}$ at $4{ }^{\circ} \mathrm{C}$. The upper aqueous layer (containing RNA) was removed and collected into a fresh RNAse-free microcentrifuge tube. Total RNA was then precipitated with the addition of $500 \mu \mathrm{L}$ of isopropanol followed by incubation for $10 \mathrm{~min}$ at RT. Samples were then centrifuged again as above. The RNA pellet was washed with $70 \%$ ethanol and centrifuged again. The supernatant was removed and tubes were allowed to dry for 10-15 min and then resuspended in $50 \mu \mathrm{L}$ DEPC-treated $\mathrm{ddH}_{2} \mathrm{O}$. RNA quality was assessed by the $260 / 280 \mathrm{~nm}$ ratio and gel electrophoresis on a $1 \%$ agarose gel stained with $2 \mathrm{X}$ Sybr Green I nucleic acid gel stain (Invitrogen; Cat\# S-7567) to check for integrity of the $18 S$ 
and $28 S$ ribosomal RNA bands (Appendix E). All RNA samples were diluted to $1 \mu \mathrm{g} / \mu \mathrm{L}$ RNA using DEPC-treated dd $\mathrm{H}_{2} \mathrm{O}$.

\subsection{5 cDNA synthesis}

A $5 \mu \mathrm{g}$ aliquot of total RNA from each sample was diluted in $10 \mu \mathrm{L}$ of DEPCtreated $\mathrm{H}_{2} \mathrm{O}$ and $1 \mu \mathrm{L}$ of $200 \mathrm{ng} / \mu \mathrm{L}$ oligo-dT (5'-TTTTTTTTTTTTTTTTTTTTTTV-3'; $\mathrm{V}=\mathrm{A}, \mathrm{G}$, or $\mathrm{C}$ ) primer was added to each PCR tube (total volume of $11 \mu \mathrm{l}$ ). The samples were incubated in a thermal cycler for $5 \mathrm{~min}$ at $65^{\circ} \mathrm{C}$. The mixture was then chilled rapidly on ice and $4 \mu \mathrm{L}$ of $5 \mathrm{X}$ first strand buffer (Invitrogen; Cat\# 28025-021), $2 \mu \mathrm{L}$ of 0.1 M dithiothreitol (DTT) (Invitrogen; Cat\#28025-021), $1 \mu \mathrm{L}$ of $25 \mathrm{mM}$ dNTPs (BioBasic; Cat\#DD0057) and $1 \mu \mathrm{L}$ of M-MLV reverse transcriptase (Invitrogen; Cat\# 28025-021) were added to each sample. The mixture was placed back into the thermal cycler and incubated for $45 \mathrm{~min}$ at $42^{\circ} \mathrm{C}$. The resulting cDNA was serial diluted to $10^{-3}$ and stored at $-20^{\circ} \mathrm{C}$.

\subsubsection{Polymerase chain reaction}

Polymerase chain reaction (PCR) was used to amplify the sequences under study from the cDNA samples. Each PCR reaction consisted of $13.25 \mu \mathrm{L}$ of sterile water, $5 \mu \mathrm{L}$ of diluted cDNA, $1.25 \mu \mathrm{L}$ of $1.5 \mu \mathrm{M}$ primer mixture (see Appendix F), $2.5 \mu \mathrm{L}$ of $10 \mathrm{X}$ PCR buffer (Invitrogen; Cat\#N8080006), $1.5 \mu \mathrm{L}$ of $50 \mathrm{mM} \mathrm{MgCl}_{2}, 0.5 \mu \mathrm{L}$ of $25 \mathrm{mM}$ dNTPs and $1 \mu \mathrm{L}$ of Taq polymerase, for a total volume of $25 \mu \mathrm{L}$. All PCR amplification cycles were as follows; an initial denaturation at $94{ }^{\circ} \mathrm{C}$ for $7 \mathrm{~min}$, followed by an experimentally determined number of cycles of $94{ }^{\circ} \mathrm{C}$ for $1 \mathrm{~min}$, primer annealing at 50$60{ }^{\circ} \mathrm{C}$ (experimentally determined for each gene; see Appendix G) for 1 min, and 
elongation at $72{ }^{\circ} \mathrm{C}$ for $1 \mathrm{~min}$. The final elongation was at $72{ }^{\circ} \mathrm{C}$ for $10 \mathrm{~min}$. PCR products were held at $4{ }^{\circ} \mathrm{C}$ after amplification.

All PCR products were separated on 1\% agarose gels stained with $2 \mathrm{X}$ SybrGreen I nucleic acid gel stain (Invitrogen; Cat\# S-7567) in $3 \mu$ of DNA loading buffer $(0.25 \%$ xylene cyanol, $30 \%$ glycerol in $\mathrm{ddH}_{2} \mathrm{O}$ ). All samples were visualized using the ChemiGenius imaging system (Syngene) under UV light and band densities were quantified using the GeneTools program. Initial trials with serially diluted cDNA samples established the appropriate dilution to use for each gene of interest (ie. the band from the most dilute cDNA sample that gave visible product). This ensured that the products had not reached amplification saturation. This cDNA dilution was then used in all subsequent runs for quantification purposes,

\subsubsection{Sequencing}

All amplification products were sequenced by BioBasics (Markham, ON). Sequences were verified as encoding the correct genes using the program BLASTN at the $\mathrm{NIH}$ (http://www.ncbi.nlm.nih.gov/blast). The sequences were translated using DNAMAN.

\subsubsection{Statistics}

Detection of bands on gels and blots used the ChemiGenius Bio-Imaging System (Syngene, Frederick, MD) and densitometric analysis was performed with the associated GeneTools software. Immunoblot band intensity in each lane was normalized against a strong Coomassie blue stained band in the same lane to correct any minor variations in 
sample loading; the Coomassie stained band chosen showed constant intensity across all samples and was well-separated from the area of the gel containing the immunoreactive protein. For RT-PCR, band intensity was normalized against the corresponding intensity of the $\alpha$-tubulin bands amplified from the same RNA sample. Mean normalized band densities \pm SEM were calculated for control and anoxic samples and significance testing using a one-way analysis of variance followed by the Student-Newman-Keuls test for the control, 5 and $20 \mathrm{~h}$ anoxia data. Statistical difference was accepted if $P<0.05$; all data are derived from multiple independent tissue extracts from different animals. 


\subsection{Results}

\subsubsection{Expression and phosphorylation of Akt}

The relative phosphorylation state of selected key residues on a protein can often be determined using an antibody that recognizes the specific short peptide sequence containing the phosphorylated amino acid residue. All Akt antibodies (both total protein and phospho-specific) cross-reacted with a protein band of $\sim 60 \mathrm{kDa}$, the expected size of Akt (Figure 2.4). Note that Figure 2.4 shows examples of immunoblot images for all proteins and phospho-proteins evaluated in this chapter. The effect of anoxia exposure for 5 or $20 \mathrm{~h}$ on Akt in liver, kidney and white skeletal muscle of turtles was evaluated (Figure 2.5). No significant changes in total Akt expression were detected in liver or kidney tissues in response to either length of anoxia whereas white skeletal muscle showed a significant increase in total Akt expression of $2.23 \pm 0.03$-fold after $20 \mathrm{~h}$ anoxia, as compared to control values $(P<0.05)$. Levels of p-Akt $(\mathrm{Thr308})$ remained stable after $5 \mathrm{~h}$ of anoxia exposure in all studied tissues. However, in kidney and white skeletal muscle p-Akt (Thr308) expression increased by $2.05 \pm 0.32$-fold and $2.01 \pm 0.23$-fold, respectively, in response to $20 \mathrm{~h}$ of anoxia (both $P<0.05$ ). In all tissues, the expression of p-Akt (Ser473) remained stable after $5 \mathrm{~h}$ of anoxia exposure but the relative amount of p-Akt (Ser473) increased significantly to $1.47 \pm 0.14$-fold and 3.79 \pm 0.46 -fold over control values in kidney and white skeletal muscle after $20 \mathrm{~h}$ anoxia, compared to control values $(P<0.05)$

\subsubsection{Expression and phosphorylation of GSK-3ß}

The activation status of GSK-3 $\beta$ is dependent upon its phosphorylation by Akt 
(Takuwa et al., 1999). Antibodies recognizing both total GSK-3 $\beta$ and p-GSK-3 $\beta$ (Ser9) cross-reacted with a protein band of $\sim 47 \mathrm{kDa}$, the expected size of GSK-3 $\beta$ (Figure 2.4). Changes in the GSK-3 $\beta$ in response to anoxia are shown in Figure 2.6. No significant changes in total GSK-3 $\beta$ expression were detected in liver or kidney tissues in response to either 5 or $20 \mathrm{~h}$ anoxia compared to control values. However, white skeletal muscle showed a significant increase in total GSK-3 $\beta$ expression of $2.31 \pm 0.14$-fold after $20 \mathrm{~h}$ anoxia, when compared to control values $(P<0.05)$. Levels of p-GSK-3 $\beta$ (Ser9) in both liver and kidney remained stable after $5 \mathrm{~h}$ of anoxia exposure, but decreased significantly in white skeletal muscle to $54 \pm 12 \%$ of control levels after $5 \mathrm{~h}$ of anoxia $(P<0.05)$. Levels of p-GSK-3 $\beta$ (Ser9) increased significantly in both kidney and white skeletal muscle after $20 \mathrm{~h}$ anoxia, rising by $1.38 \pm 0.07$-fold and $1.78 \pm 0.31$-fold, respectively, compared to control values $(P<0.05)$.

To assess the downstream activity of GSK-3 $\beta$ on regulating the cell cycle through Cyclin D1, the relative phosphorylation of p-Cyclin D1 (Thr284) was assessed. Figure 2.6 shows that in response to 5 and $20 \mathrm{~h}$ anoxia, the relative phosphorylation of $\mathrm{p}$ Cyclin D1 (Thr284) in liver decreased to $78 \pm 5$ and $79 \pm 1 \%$ of control values, respectively $(P<0.05)$. In kidney tissue, the relative levels of p-Cyclin D1 (Thr284) only decreased after $20 \mathrm{~h}$ anoxia, significantly declining to $63 \pm 5 \%$ of control values $(P<0.05)$. No significant changes in the relative levels of p-Cyclin D1 (Thr284) occurred under anoxia in white skeletal muscle. 


\subsubsection{Total expression of Cyclin proteins}

Expression levels of four Cyclin proteins (D1, E1, A1, B1) revealed similar trends in liver, kidney and white skeletal muscle of turtles in response to 5 and $20 \mathrm{~h}$ anoxia exposure (Figure 2.7). Cyclin D1 (immunoreactive band of $\sim 38 \mathrm{kDa}$; Figure 2.4) protein decreased significantly under both 5 and $20 \mathrm{~h}$ anoxia to $59 \pm 1 \%$ and $61 \pm 9 \%$ of control values in liver tissue, respectively $(P<0.05)$. In kidney, total protein expression of Cyclin D1 decreased after $20 \mathrm{~h}$ anoxia to $66 \pm 8 \%$ of control values $(P<0.05)$ but no significant change in Cyclin D1 expression was detected in white skeletal muscle.

Cyclin E1 protein (immunoreactive band of $\sim 47 \mathrm{kDa}$; Figure 2.4) decreased significantly under both 5 and $20 \mathrm{~h}$ anoxia to $59 \pm 3$ and $72 \pm 2 \%$ of control values in liver tissue, respectively $(P<0.05)$. In kidney, however, was Cyclin E1 decreased significantly only after $20 \mathrm{~h}$ anoxia to $68 \pm 8 \%$ of control values $(P<0.05)$. No significant changes in Cyclin E1 expression were detected in white skeletal muscle.

Cyclin A1 (immunoreactive band of $\sim 54 \mathrm{kDa}$; Figure 2.4) protein decreased significantly under both 5 and $20 \mathrm{~h}$ anoxia in kidney and white skeletal muscle. In kidney tissue, exposure to 5 and $20 \mathrm{~h}$ anoxia reduced Cyclin A1 levels to $58 \pm 4$ and $51 \pm 7 \%$ of control values, respectively $(P<0.05)$; comparable values in white skeletal muscle were $51 \pm 14$ and $57 \pm 4 \%$ of controls, respectively $(P<0.05)$. In liver, protein expression of Cyclin A1 declined significantly only after $20 \mathrm{~h}$ anoxia, decreasing to $57 \pm 4 \%$ of control values $(P<0.05)$.

Cyclin B1 (immunoreactive band of $\sim 48 \mathrm{kDa}$; Figure 2.4) protein levels increased significantly in liver tissue after $5 \mathrm{~h}$ anoxia by $1.48 \pm 0.06$-fold compared to control values, however after $20 \mathrm{~h}$ anoxia levels had returned to control values $(P<0.05)$. Similar 
changes in Cyclin B1 expression were detected in kidney; levels of Cyclin Blincreased significantly after $5 \mathrm{~h}$ anoxia by $1.42 \pm 0.09$-fold compared to control values, but returned to control values after $20 \mathrm{~h}(P<0.05)$. In white skeletal muscle, the protein expression of Cyclin B1 only increased significantly after $20 \mathrm{~h}$ of anoxic exposure, rising to $1.77 \pm 0.15$ fold higher than control values $(P<0.05)$.

\subsubsection{Nuclear expression of Cyclins}

Cyclins dynamically shuttle between nuclear and cytoplasmic environments. Expression in nuclear fractions is critical, as this is the location of primary functions related to the cell cycle. Expression levels of cyclin proteins in nuclear fractions are shown in Figure 2.8 and revealed similar expression trends to those seen above for total cyclin levels. Cyclin D1 nuclear protein decreased significantly under both 5 and $20 \mathrm{~h}$ anoxia to $50 \pm 5$ and $66 \pm 6 \%$ of control values in liver tissue, respectively $(P<0.05)$. A decrease in protein expression of nuclear Cyclin D1 was also detected in kidney tissues, decreasing after $20 \mathrm{~h}$ anoxia to $55 \pm 9 \%$ of control values $(P<0.05)$. No significant change in the nuclear expression of Cyclin D1 occurred in white skeletal muscle in response to anoxia.

Cyclin E1 nuclear protein in liver decreased significantly only after $20 \mathrm{~h}$ anoxia to $38 \pm 2 \%$ of control values $(P<0.05)$. Significant decreases in nuclear protein expression of Cyclin E1 were detected in kidney tissues, decreasing during 5 and $20 \mathrm{~h}$ anoxia to $72 \pm 6$ and $57 \pm 4 \%$ of control values, respectively $(P<0.05)$. No significant change in nuclear Cyclin E1 expression was detected in white skeletal muscle. 
Cyclin A1 nuclear protein increased significantly after $5 \mathrm{~h}$ anoxia in liver, kidney and white skeletal muscle by $1.53 \pm 0.01$-fold, $1.40 \pm 0.05$-fold and $1.38 \pm 0.03$-fold compared to control values, respectively $(P<0.05)$. After exposure to $20 \mathrm{~h}$ of anoxia, nuclear expression of Cyclin A1 protein remained high in liver and kidney at $1.61 \pm 0.17$ fold and 1.70 \pm 0.17 -fold over control values, respectively $(P<0.05)$ but nuclear Cyclin A1 protein expression in white skeletal muscle returned to control values $(P<0.05)$.

The nuclear expression of Cyclin B1 protein displayed similar expression patterns to that seen for Cyclin D1. Cyclin B1 nuclear protein levels decreased significantly under both 5 and $20 \mathrm{~h}$ anoxia to $72 \pm 4$ and $46 \pm 3 \%$ of control values in liver tissue, respectively $(P<0.05)$. Similar decreases in nuclear Cyclin B1 were detected in kidney, decreasing during 5 and $20 \mathrm{~h}$ anoxia to $67 \pm 6$ and $32 \pm 4 \%$ of control values, respectively $(P<0.05)$. No significant changes in nuclear Cyclin B1 were detected in white skeletal muscle in response to anoxia.

\subsubsection{Transcript expression of Cyclins}

Using RT-PCR and primers derived from the consensus sequences of cyclins $d 1$, $e 1, a l$ and $b 1$ obtained from other vertebrates, relative expression of cyclin transcripts were assessed in response to anoxia in the turtle (Figure 2.9). No significant changes in the expression of cyclin $d 1$ transcript levels were detected in response to either 5 or $20 \mathrm{~h}$ anoxia in any tissue. The amplified transcript and associated translated protein sequence for the turtle cyclin $d 1$ amplicon is presented in Figure 2.10A. The amplified turtle sequence represented $38 \%$ mRNA coverage and displayed $64 \%$ homology to the corresponding human sequence (Figure 2.10B). The translated turtle protein sequence 
represented $86 \%$ coverage and displayed $83 \%$ homology to the corresponding human protein sequence (Figure 2.10C).

Relative transcript expression of cyclin el decreased significantly after both 5 and $20 \mathrm{~h}$ anoxia to $12 \pm 3$ and $23 \pm 1 \%$ of control values in liver tissue, respectively $(P<0.05)$. However, no significant changes in cyclin el transcript expression were detected in either kidney or white muscle after either $5 \mathrm{~h}$ or $20 \mathrm{~h}$ anoxia. The amplified cyclin el transcript and translated protein sequence for the cyclin el amplicon are presented in Figure 2.11A. The amplified turtle sequence represented 30\% mRNA coverage and displayed 68\% homology to the corresponding human sequence (Figure 2.11B). The translated turtle protein sequence represented $46 \%$ coverage and displayed $75 \%$ homology to the corresponding human protein sequence (Figure 2.11C).

No significant changes in the expression of cyclin al transcript levels were detected in response to either 5 or $20 \mathrm{~h}$ anoxia in liver or kidney tissues. However, relative transcript expression of cyclin al significantly increased strongly in white skeletal muscle by $3.49 \pm 0.41$ and $5.12 \pm 0.09$-fold compared to control values, in response to 5 and $20 \mathrm{~h}$ anoxia, respectively $(P<0.05)$ (Figure 2.9). The amplified transcript and translated protein sequence for the cyclin al amplicon are presented in Figure 2.12A. The amplified turtle sequence represented 12\% mRNA coverage and displayed $59 \%$ homology to the corresponding human sequence (Figure 2.12B). The translated turtle protein sequence represented $26 \%$ coverage and displayed 56\% homology to the corresponding human protein sequence (Figure 2.12C).

Patterns of cyclin b1 expression were highly tissue specific. A significant decrease in cyclin b1 transcript expression to $43 \pm 6 \%$ of control values was detected in liver tissue 
after $5 \mathrm{~h}$ anoxia, however expression increased strongly by $2.83 \pm 0.33$-fold compared to control values after $20 \mathrm{~h}$ anoxia $(P<0.05)$. In kidney, relative transcript expression of cyclin bl increased significantly after both 5 and $20 \mathrm{~h}$ anoxia by $1.90 \pm 0.12$ and $2.61 \pm 0.32$-fold compared to control values, respectively $(P<0.05)$. A significant increase in cyclin bl transcript expression was detected after $5 \mathrm{~h}$ anoxia, increasing by $2.34 \pm 0.32$ fold compared to control values in white muscle tissue $(P<0.05)$. However, after $20 \mathrm{~h}$ anoxia, transcript expression of cyclin bl decreased again, back to control levels $(P<$ 0.05). The amplified transcript and translated protein sequence for the cyclin bl amplicon is presented in Figure 2.13A. The amplified turtle sequence represented 23\% mRNA coverage and displayed 79\% homology to the corresponding human sequence (Figure 2.13B). The translated turtle protein sequence represented $37 \%$ coverage and displayed $76 \%$ homology to the corresponding human protein sequence (Figure 2.13C).

\subsubsection{Total expression of Cdks}

Changes in the relative level of total and phosphorylated forms of several cyclindependent kinases $(\mathrm{Cdk})$ were assessed in response to anoxia using immunoblotting (Figure 2.14). Cdk4 protein (immunoreactive band of $\sim 35 \mathrm{kDa}$; Figure 2.4) decreased under anoxia in all three tissues tested. Levels fell significantly under both 5 and $20 \mathrm{~h}$ anoxia to $51 \pm 7$ and $69 \pm 5 \%$ of control values in liver tissue, respectively $(P<0.05)$. In kidney, Cdk4 protein levels fell during 5 and $20 \mathrm{~h}$ anoxia to $82 \pm 1$ and $79 \pm 1 \%$ of control values, respectively $(P<0.05)$; comparable decreases in white skeletal muscle were $71 \pm 4$ and $51 \pm 14 \%$ of control values, respectively $(P<0.05)$. 
Cdk6 (immunoreactive band of $\sim 35 \mathrm{kDa}$; Figure 2.4) protein did not change significantly in response to anoxia in either kidney or white skeletal muscle tissues. In liver, however, the relative expression of Cdk6 decreased after $20 \mathrm{~h}$ anoxia to $61 \pm 8 \%$ of control levels $(P<0.05)$.

Similar to anoxic response of $\mathrm{Cdk} 6$, the protein expression of $\mathrm{Cdk} 2$ (immunoreactive band of $\sim 34 \mathrm{kDa}$; Figure 2.4) did not significantly change in response to anoxia in either kidney or white skeletal muscle. In liver tissue, the relative expression of Cdk2 decreased only after $20 \mathrm{~h}$ anoxia, falling to $62 \pm 2 \%$ of control levels $(P<0.05)$.

Cdc2 protein (immunoreactive band of $\sim 34 \mathrm{kDa}$; Figure 2.4 ) expression did not change significantly in response to anoxia in liver tissue. Total protein expression of Cdc2 did increase significantly in kidney after $20 \mathrm{~h}$ anoxia by $1.28 \pm 0.03$-fold compared to control values $(P<0.05)$ whereas in skeletal muscle Cdc2 expression significantly decreased after $20 \mathrm{~h}$ anoxia to $62 \pm 10 \%$ of control values $(P<0.05)$.

The combined phosphorylation state of all Cdks can be determined using a specific anti-p-Cdk (Thr14/Thr15) antibody (immunoreactive band of $\sim 35 \mathrm{kDa}$; Figure 2.4). The relative phosphorylation state of $\mathrm{p}-\mathrm{Cdk}(\mathrm{Thr} 14 / \mathrm{Thr} 15)$ increased significantly in all tissues in response to both 5 and $20 \mathrm{~h}$ anoxia. Relative phosphorylation levels increased by $2.29 \pm 0.10,1.94 \pm 0.03$ and $1.62 \pm 0.11$-fold compared to control values after 5 h of anoxia exposure for liver, kidney and white skeletal muscle, respectively $(P<0.05)$. Similarly, the expression of p-Cdk (Thr14/Thr15) remained high in all three tissues at $2.25 \pm 0.13,1.66 \pm 0.07$ and $2.08 \pm 0.05$-fold higher than control values after $20 \mathrm{~h}$ of anoxia exposure for liver, kidney and white skeletal muscle, respectively $(P<0.05)$. 


\subsubsection{Nuclear expression of Cdks}

Similar to Cyclins, Cdks are also dynamically shuttled between nuclear and cytoplasmic environments. Protein expression in nuclear fractions is crucial, as this is the primary location of cell cycle related functions. Expression levels of Cdk proteins in nuclear fractions did not show similar expression trends under anoxia as seen in total cell extracts, suggesting differential regulation of Cdk activity by subcellular distribution (Figure 2.15). In liver tissue, the nuclear protein expression of Cdk4 did not significantly change in response to anoxia. In kidney, nuclear expression of Cdk4 decreased to $47 \pm 6$ and $54 \pm 3 \%$ of control values in response to 5 and $20 \mathrm{~h}$ anoxia, respectively $(P<0.05)$ whereas, in white skeletal muscle nuclear Cdk4 content decreased to $51 \pm 11$ and $64 \pm 12 \%$ of control values, respectively $(P<0.05)$.

The nuclear expression of Cdk6 in liver significantly decreased after exposure to $20 \mathrm{~h}$ anoxia, decreasing to $71 \pm 8 \%$ of control values $(P<0.05)$. In kidney, the nuclear expression of Cdk6 decreased even further to $25 \pm 3 \%$ of control values upon exposure to $20 \mathrm{~h}$ anoxia $(P<0.05)$ whereas the nuclear levels of Cdk6 in white skeletal muscle decreased to $65 \pm 8 \%$ of control values $(P<0.05)$. No significant changes in nuclear Cdk6 protein expression occurred in response to $5 \mathrm{~h}$ anoxia in any tissue.

Nuclear Cdk2 protein expression decreased significantly only after $20 \mathrm{~h}$ anoxia in both liver and kidney, falling to $74 \pm 7$ and $11 \pm 6 \%$ of control values, respectively $(P<$ 0.05). In white skeletal muscle, the relative nuclear levels Cdk2 did not significantly change in response to anoxia.

Nuclear Cdc2 protein expression decreased significantly only after $20 \mathrm{~h}$ anoxia in liver, kidney and white skeletal muscle, decreasing to $44 \pm 13,68 \pm 8$, and $55 \pm 4 \%$ of control 
values, respectively $(P<0.05)$. No significant changes in nuclear $\mathrm{Cdc} 2$ protein expression were found in response to $5 \mathrm{~h}$ anoxia in any tissue studied.

\subsubsection{Transcript expression of Cdks}

Using RT-PCR and primers designed from the consensus sequences of $c d k 4,6,2$ and $c d c 2$ from other vertebrates, the relative expression of $c d k$ and $C d c 2$ transcripts were assessed in response to anoxia in the turtle (Figure 2.16). The relative transcript expression of $c d k 4$ did not significantly change in response to anoxia in either liver or muscle. In kidney, however, a significant decrease in $c d k 4$ transcript expression was detected after $5 \mathrm{~h}$ anoxia, decreasing to $52 \pm 3 \%$ of control values $(P<0.05)$ but rebounding after $20 \mathrm{~h}$ anoxia. The amplified transcript and associated translated protein sequence for the $c d k 4$ amplicon is presented in Figure 2.17A. The amplified turtle sequence represented 9\% mRNA coverage and displayed 69\% homology to the corresponding human sequence (Figure 2.17B). The translated turtle protein sequence represented $19 \%$ coverage and displayed $83 \%$ homology to the corresponding human protein sequence (Figure 2.17C).

Relative transcript expression of $c d k 6$ did not significantly respond to anoxia in any of the three tissues studied. The amplified transcript and associated translated protein sequence for the $c d k 6$ amplicon is presented in Figure 2.18A. The amplified turtle sequence represented $22 \%$ mRNA coverage and displayed $78 \%$ homology to the corresponding human sequence (Figure 2.18B). The translated turtle protein sequence represented 56\% coverage and displayed $91 \%$ homology to the corresponding human protein sequence (Figure 2.18C). 
Relative transcript expression of $c d k 2$ did not significantly change in response to anoxia in liver tissue. Relative expression of $c d k 2$ transcripts in kidney decreased significantly in response to both 5 and $20 \mathrm{~h}$ anoxia, falling to $43 \pm 10$ and $73 \pm 7 \%$ of control values, respectively $(P<0.05)$. In contrast, relative transcript expression of $c d k 4$ increased significantly in response to both 5 and $20 \mathrm{~h}$ anoxia in white skeletal muscle, increasing by $3.58 \pm 0.54$ and $9.13 \pm 0.96$-fold compared to control values, respectively ( $P$ $<0.05)$. The amplified transcript and associated translated protein sequence for the $c d k 2$ amplicon is presented in Figure 2.19A. The amplified turtle sequence represented 51\% mRNA coverage and displayed $67 \%$ homology to the corresponding human sequence (Figure 2.19B). The translated turtle protein sequence represented $84 \%$ coverage and displayed 93\% homology to the corresponding human protein sequence (Figure 2.19C). Relative transcript expression of $c d c 2$ decreased significantly only after $20 \mathrm{~h}$ anoxia in both liver and kidney tissue, decreasing to $56 \pm 2$ and $72 \pm 5 \%$ of control values, respectively $(P<0.05)$. However, no significant changes in the expression of cdc2 transcript levels were detected after either 5 or $20 \mathrm{~h}$ anoxia in white skeletal muscle. The amplified transcript and associated translated protein sequence for the $c d c 2$ amplicon is presented in Figure 2.20A. The amplified turtle sequence represented $43 \%$ mRNA coverage and displayed $74 \%$ homology to the corresponding human sequence (Figure 2.20B). The translated turtle protein sequence represented 39\% coverage and displayed $72 \%$ homology to the corresponding human protein sequence (Figure 2.20C). 


\subsection{Discussion}

Extreme hypoxia is central to a variety of diseases including cardiac and pulmonary dysfunction, as well as tumor progression (Lipton, 1999). Although cancers are incredibly diverse, researchers have been searching for a small number of underlying controls, whose activity is required for the development of all cancers (Bonetta, 2005). Many of these studies examine cell cycle components as key players in the uncontrollable proliferation and the progression of tumor growth. Cell cycle regulation in hypoxic environments, such as that of tumor cores, has shown great potential to expose the secrets of cell cycle arrest. Given this, there has been an overwhelming interest in the elucidation of the molecular mechanisms regulating survival in various levels of oxygen deprivation (Padilla et al., 2002). Many of these studies focus on hypoxia tolerant animal models and cell lines of various tolerances to oxygen restriction. Studies examining the characteristics of cell cycle regulation under hypoxia in brine shrimp (Artemia franciscana) embryos, zebrafish (Danio rerio) embryos, nematodes (Caenorhabditis elegans), and fruit flies (Drosophila melanogaster) have shown that specific stages exist in which the cell cycle can arrest during periods of hypoxia; however, the exact mechanism of arrest seems to be species specific (Padilla et al., 2002; Clegg, 1997; Hand, 1998; Padilla and Roth, 2001; Douglas and Haddad, 2003). Although arrest pathways appear to be highly conserved throughout evolution, examination of a higher vertebrate animal model may prove to be useful in elucidating general mechanisms of vertebrate cell cycle arrest in response to oxygen restriction.

The regulation of cellular proliferation (cell cycle) is of particular interest to the field of anoxia tolerance because of its metabolic costs during periods of hypometabolism 
and of its previous mentioned implication to health science research. To better understand the regulation of the cell cycle during anoxia, the present study investigated the regulation of important protein regulators of the cycle, in response to anoxic submergence in the red eared slider turtle, T. scripta elegans. The cell cycle is hierarchal in its regulatory structure, where the expression of proteins is regulated in a temporal manner and initiates the expression of the next proteins necessary for upcoming processes (Harbour et al., 1999). Because of this structure, it is possible to approximate whether proliferating cells are able to respond to anoxia and if these cells arrest at any particular checkpoint. Important to this study, is the phase-specific expression of Cyclin and Cdk proteins (Figure 2.1).

\subsubsection{Regulation of Akt signaling and cell cycle control}

The regulation of the Akt signaling pathway is of importance to regulating the cell cycle in response to extracellular stimuli (Chang et al., 2003). Activation of Akt, measured indirectly by relative levels of Thr308 and Ser473 phosphorylation, is able to activate the cell cycle through several critical mechanisms. One mechanism is via the indirect prevention of Cyclin D1 degradation, a protein responsible for initiating the cell cycle. This action is facilitated by the direct inactivation of the upstream kinase, GSK-3 $\beta$, by Akt (Dal Col and Dolcetti, 2008). During periods when the cell cycle needs to be suppressed, active GSK-3 $\beta$ is able to phosphorylate Cyclin D1 (Thr284), targeting that protein for poly-ubiquitination and subsequent proteolysis. Active GSK-3 $\beta$ usually inhibits the activity of its downstream targets and is known to regulate a number of other intracellular pathways, including glycogen storage and apoptosis (Franke et al., 2003). 
Another regulatory mechanism for Akt control of the cell cycle is through both direct and indirect interactions with CKIs, p21 and p27 (Figure 2.2). The mechanism of regulation for these CKIs by Akt will be discussed in further detail in Chapter 3, but typically leads to the inhibition of CKI activity.

Evidence of increased Akt activity in response to anoxia in turtles was detected in both kidney and white skeletal muscle (Figure 2.5). Both tissues displayed a significant increase in relative phosphorylation levels (both Thr308 and Ser473) of the kinase in response to $20 \mathrm{~h}$ anoxia, indicating a relative increase in active Akt protein. Both tissues also showed an increase in the relative phosphorylation of p-GSK-3 $\beta$ (Ser9) (Figure 2.6), suggesting that Akt is indeed active and inhibiting GSK-3 $\beta$ function. Notably, as a result of decreased GSK-3 $\beta$ function, the relative phosphorylated state of p-Cyclin D1 (Thr284) in kidney appropriately decreased in response to $20 \mathrm{~h}$ anoxia; however, $\mathrm{p}$-Cyclin D1 (Thr284) did not change in in white skeletal muscle (Figure 2.6). Interestingly, the ratio of total Cyclin D1 protein to p-Cyclin D1 (Thr284) did not change in any studied tissue, indicating that the upstream kinase was not active and the relative phosphorylation of pCyclin D1 (Thr284) did not increase during anoxia (see Chapter 5; Figure 5.4B). However, an increase in Akt phosphorylation does not necessarily indicate an activation of the cell cycle, since many alternate mechanisms contribute to overall cell cycle control and Akt may regulate many other cellular processes during anoxia. One alternative role for Akt is the regulation of glucose metabolism. Interestingly, the present study found no indication of Akt activation in liver during anoxia. 


\subsubsection{Regulation of Cyclins in response to anoxia}

The ordered progression through cell cycle phases is controlled by the sequential expression of Cyclins. Both Cyclin D1 and Cyclin E1 are highly expressed during the $\mathrm{G}_{1}$ phase of the cell cycle, while Cyclin A1 and Cyclin B1 are expressed during the $\mathrm{S}$ and $\mathrm{G}_{2}$ phases, respectively. In response to anoxia, both liver and kidney showed similar overall reductions in the relative amounts of Cyclins D1, E1 and A1. Although it is difficult to associate changes in cell cycle activity with general changes in the expression of these Cyclins, the results indicate that the cell cycle may be (1) arresting, (2) preparing for Mphase entry, or (3) undergoing a general reduction in proliferation rate. White skeletal muscle, a tissue with little proliferative potential, displayed no significant changes in $\mathrm{G}_{1}$ Cyclins (type D1 and E1), but showed a decrease in Cyclin A1 in response to 5 and $20 \mathrm{~h}$ anoxia.

The function of Cyclins on the cell cycle is limited to the nucleus, where many cell cycle related processes occur. The nuclear expression of Cyclin D1 decreased in response to anoxia in both liver and kidney but was unchanged in muscle (Figure 2.8). The nuclear expression of Cyclin E1 showed the same pattern. Interestingly, the relative amounts of nuclear Cyclin A1 significantly increased after $5 \mathrm{~h}$ anoxia in all studied tissues and remained elevated after $20 \mathrm{~h}$ anoxia in liver and kidney. The increase in nuclear translocation of Cyclin A1 during anoxia is surprising given the results for all other Cyclins. The typical nuclear function of Cyclin A1 protein is to create a complex with Cdk2 and activate the replication of DNA. However, alternate roles for the Cyclin A1/Cdk2 complex exist and may be utilized by the turtle during anoxia. Cyclin A1/Cdk2 complex has been shown to phosphorylate E2F1 which inactivates DNA binding and 
transactivation by E2F1 (Kitagawi et al., 1995). Thus two mechanisms exist whereby Cyclin A1 is able to either activate or inhibit the progression of the cell cycle. To fully elucidate the function of Cyclin A1 it is necessary to study the regulation of its associated cyclin-dependent kinases and inhibitors (See below and Chapter 3). The possibility of Cyclin A1/Cdk2 phosphorylation control over E2F1 and its downstream effects on DNA binding in the anoxic turtle are also explored (Chapter 4).

The expression of Cyclin mRNA transcripts was measured to further determine possible modes of their regulation (transcriptional, post-transcriptional or posttranslational). Despite a decrease in Cyclin D1 protein expression to some degree (both total and nuclear) in liver and kidney (Figure 2,7 and 2.8) and no increase in apparent proteasomal degradation of Cyclin D1, as indicated by overall reduced Tyr284 phosphorylation in these two tissues (Figure 2.6), no change in cyclin d1 mRNA levels were observed in any studied tissue (Figure 2.9). This regulation pattern suggests a possible post-transcriptional mode of Cyclin D1 regulation during anoxia. Indeed, it has been reported that mutations occurring in the 3' UTR of cyclin $d 1$ are responsible for the post-transcriptional regulation of cyclin $d 1$ in a variety of tumour types (Wiestner et al., 2007). In this regard, studies examining the effect of proliferative rate with cells expressing cyclin $d 1$ lacking the 3' UTR found a significantly higher rate of protein expression and cellular proliferation (Wiestner et al., 2007; Deshpande et al., 2009). These studies suggest the presence of negative regulatory elements (such as microRNA binding sites) within the 3' UTR of cyclin $d 1$ that aid in the regulation of Cyclin D1 protein expression. Interestingly, the 3'UTR of cyclin 11 may be critical to Cyclin D1 
regulation and as a result may be important in regulating cellular proliferation during periods of cellular stress (see Chapter 5).

The expression of cyclin el transcripts was similar to the expression profile of total protein Cyclin E1 in all studied tissues. This finding suggests a probable transcriptional regulation of Cyclin E1 protein, likely by the E2F1 transcription factor (Weinmann et al., 2001). Similar to cyclin $d 1$, the expression of cyclin al transcripts displayed no change in response to anoxia in liver and kidney (Figure 2.9) although total Cyclin A1 protein decreased in both tissues. This could indicate a possible increase in Cyclin A1 protein degradation or possible post-transcriptional regulation of cyclin a1 mRNA in response to anoxia. Uniquely, however, a strong increase in cyclin al transcript levels occurred in skeletal muscle although Cyclin A1 protein content decreased under anoxia. This indicates a clear disjoint between transcription and translation of this protein in anoxic muscle. Indeed, several RNA binding proteins (RBPs) have been shown to negatively regulate the translation of Cyclin A1 protein. These proteins include the HuR family of RBPs that bind to the cis-elements within the 3' UTR of cyclin al containing HuR binding elements (Wang et al., 2000). Binding of HuR proteins to cyclin al have been shown to increase transcript half-life during periods of cellular arrest. Similar to cyclin $d 1$, cyclin al could be under RBP or microRNA post-transcriptional control during anoxia in the turtle. Further studies in this area are needed to explore this possibility.

Interestingly, the expression profile of Cyclin B1 protein was not similar to any other measured Cyclin. This is surprising as the increased expression of Cyclin B1 is dependent on the expression and associated Cdk activities of Cyclin D1 and Cyclin E1. Increased activity in the Cyclin/Cdk complexes associated with type D1 and E1 Cyclins 
are necessary for the activation of the E2F1 transcription factor and subsequent expression of cyclin b1 mRNA and protein. However, the over-expression of Cyclin B1 protein and mRNA may be a result of cell cycle independent transcriptional activation by the p53 transcription factor. Indeed, previous studies exploring the regulation of p53 in anoxic T. scripta elegans have shown an increase in p53 transcriptional activity in liver and white skeletal muscle; its effects in kidney tissue were not explored (Zhang et al., 2013). Interestingly, although both the total levels of Cyclin B1 protein and mRNA increased in response to anoxia, the nuclear localization of Cyclin B1 was found to decrease. This finding is crucial since the cell cycle-related functions of Cyclin B1 are restricted to within the nucleus. Previous studies have documented a cytoplasmic accumulation of Cyclin B1 protein during interphase of the cell cycle. Additionally, studies of hypoxic cell cycle arrest in D. melanogaster embryos reported a surprising increase in Cyclin B1 protein during a $\mathrm{G}_{1}$-like arrest of the cell cycle (Douglas, 2005).

\subsubsection{Regulation of Cdks in response to anoxia}

Although the expression profiles of Cyclins are useful in determining phasesynchronization, arrest or slowing down of the cell cycle, it is their association with Cdks that dictate their cell cycle-related activity. In all tissues studied, total expression of all Cdk proteins either significantly decreased or did not change in response to 5 or $20 \mathrm{~h}$ anoxia, except for a small increase in Cdc2 after $20 \mathrm{~h}$ anoxia in kidney (Figure 2.14). Importantly, since cell cycle-related functions of $\mathrm{Cdk}$ proteins are restricted to their presence in the nucleus, the nuclear expression of these proteins was also assessed. In response to either 5 or $20 \mathrm{~h}$ anoxia, virtually all $\mathrm{Cdk}$ proteins showed a significantly 
decreased nuclear presence in all three tissues; the exceptions were Cdk4 in liver and Cdk2 in skeletal muscle. Interestingly, nuclear Cdk expression displayed the largest decreases in kidney tissue. This is perhaps a reflection of the kidney's proliferative potential in comparison to either liver or post-mitotic white skeletal muscle tissue: cell turnover times in rats have been established to be $\sim 150$ days for kidney, compared to $\sim 400$ days for liver (comparable rates have not been established for reptiles) (Cameron, 1971). The greater turnover times in kidney tissue may establish a requirement for a stronger regulatory cell cycle response to anoxia. Apart from protein expression and subcellular location, the regulation of Cdk activity can be imparted through Wee1 kinase phosphorylation of all Cdks at either residue Thr14 (Cdk4 and Cdk6) or Tyr15 (Cdk2 and Cdc2). Cytoplasmic phosphorylation of Cdks at either of these residues halts nuclear localization and prevents their cell cycle functions. In all tissues studied, the relative phosphorylation of p-Cdk (Thr14/Tyr15) (assessing all Cdks) increased 2.0-2.5 fold in response to both 5 and $20 \mathrm{~h}$ anoxia. As phosphorylation of these residues is restricted to the cytoplasm, the relative nuclear expression of this phosphorylation event could not be established. These results present a model whereby the activity of Cdks are regulated through a decrease in both total protein levels and a reduction in nuclear localization, likely established as a result of increased p-Cdk (Thr14/Tyr15) phosphorylation.

Apart from direct regulation of Cdk proteins by either cellular localization or post-translational modifications, the relative expression of Cdk transcripts was also measured to detect possible transcriptional regulation of these targets. In response to anoxia, the relative expression of $\mathrm{G}_{1}$ phase $c d k$ (type 4 and 6) mRNAs did not change significantly during anoxia for any studied tissue with the one exception of a decrease in 
$c d k 4$ transcript levels in $5 \mathrm{~h}$ anoxic kidney. The expression of $\mathrm{G}_{1} / \mathrm{S}$ and $\mathrm{G}_{2}$ phase $c d k s$ generally decreased significantly in response to anoxia in both liver $(c d c 2)$ and kidney ( $c d k 2$ and $c d c 2$ ) tissue. As both Cdk4 and Cdk6 are important for the initiation of the cell cycle, regarding their interaction with Cyclin D1, perhaps expression levels of either mRNA are maintained during long-term anoxia in order to allow rapid translation of protein and re-entry into the cell cycle when anoxic conditions are lifted. The translation of both $c d k 4$ and $c d k 6$ transcripts into proteins could be suppressed by mature microRNA (miR-372 and miR-24, respectively) (Tian et al., 2011). Indeed, most cells are able to resume DNA synthesis within $10 \mathrm{~min}$ to $3 \mathrm{~h}$ after reoxygenation from severe hypoxia (Wilson et al., 1990). Interestingly, although no significant changes in the relative expression of $c d k 4, c d k 6$ and $c d c 2$ transcripts were detected in response to anoxia in white skeletal muscle, the relative expression of $c d k 2$ transcripts increased strongly by 3.6-fold and 9.1-fold in response to 5 or $20 \mathrm{~h}$ anoxia, respectively. Since the total protein expression of $\mathrm{Cdk} 2$ did not follow the same trend, it could be hypothesized that $c d k 2$ may be post-transcriptionally regulated in white skeletal muscle similar to $c d k 4$ and $c d k 6$.

\subsubsection{Conclusions}

In conclusion, the present study provides a summary of the critical Cyclins and Cdks involved in driving the cell cycle, as well as the activation of signaling pathways that control cell cycle initiation. There is a clear overall decrease in the relative expression of total and nuclear Cyclin and Cdk proteins in response to anoxia. This may be controlled, in part, by an initial decrease in cell cycle signaling via the Akt pathway and Cyclin D1 regulation. Although it is clear from the results that the cell cycle is 
suppressed in the organs of anoxic turtles, it is still unclear whether these results are due to phase-specific arrest mechanisms. Further study of phase-specific arrest mechanisms and not cell cycle drivers are needed and this is addressed in subsequent chapters. Additionally, although this study established the regulation of cell cycle components individually, it is still unclear how their downstream activities are affected. Subsequent chapters explore the regulation of Cdk activators ( $\mathrm{Cdc} 25 \mathrm{a}$ and $\mathrm{Cdc} 25 \mathrm{c})$ and inhibitors (p16, p27 and p21) in response to anoxia (Chapter 3). The downstream effects of changes in Cyclin/Cdk activity (Chapter 4) and post-transcriptional regulation of key cell cycle components by microRNAs (Chapter 5) are also addressed. Together, all these factors will lead to a complete understanding of the cell cycle regulatory mechanisms at play during anoxia survival in T. scripta elegans. 


\section{References}

Bonetta, L. 2005. Going on a cancer gene hunt. Cell, 123, 735-737.

Boylan, J.F., Sharp, D.M., Leffet, L., Bowers, A., and Weijun, P. 1999. Analysis of sitespecific phosphorylation of the retinoblastoma protein during cell cycle progression. Exp. Cell. Res., 248, 110-114.

Brooks, S.P.J., and Storey, K.B. 1989. Regulation of glycolytic enzymes during anoxia in the turtle Pseudemys scripta. Am. J. Physiol., 257, R278-R283.

Calin, G.A., Dumitru, C.D., Shimizu, M., Bichi, R., Zupo, S., Noch, E., Alder, H., Rattan, S., Keating, M., Rai, K., Rassenti, L., Kipps, T., Negrini, M., Bullrich, F., and Croce, C.M. 2002. Frequent deletions and down-regulation of micro-RNA genes miR15 and miR16 at13q14 in chronic lymphocytic leukemia. Proc. Natl. Acad. Sci. U.S.A. 99, 15524-15529.

Cameron, I.L. 1971. Cellular and molecular renewal in the mammalian body. Cameron, I.L., and Thrasher, J.D. eds., New York, Academic Press.

Chang, F., Lee, J.T., Navolanic, P.M., Steelman, L.S., Shelton, J.G., Blalock, W.L., Franklin, R.A., and McCubrey, J.A. 2003. Involvement of PI3K/Akt pathway in cell cycle progression, apoptosis, and neoplastic transformation: a target for cancer chemotherapy. Leukemia, 17, 590-603.

Chekulaeva, M., Hentze, M.W., and Ephrussi, A. 2006. Bruno acts as a dual repressor of oskar translation, promoting mRNA oligomerization and formation of silencing particles. Cell, 124, 521-533.

Chen, R.W., Bemis, L.T., Amato, C.M., Myint, H., Tran, H., Birks, D.K., Eckhardt, S.G., and Robinson, W.A. 2008. Truncation in CCND1 mRNA alters miR-16-1 regulation in mantle cell lymphoma. Blood, 112, 822-829.

Clegg, J.S. 1997. Embryos of Artemia franciscana survive four years of continuous anoxia: the case for complete metabolic rate depression. J. Exp. Biol., 200, 467475.

Dal Col, J., and Dolcetti, R. 2008. GSK-3b inhibition at the crossroad between Akt and mTOR constitutive activation to enhance cyclin D1 protein stability in mantle cell lymphoma. Cell Cycle, 7(18), 2813-2816.

Deshpande, A., Pastore, A., Deshpande, A.J., Zimmermann, Y., Hutter, G., Weinkauf, M., Buske, C., Hiddemann, W., and Dreyling, M. 2009. 3' UTR mediated regulation of the cyclin D1 proto-oncogene. Cell Cycle, 8(21), 3584-3592.

Douglas, R., and Haddad, G. 2003. Effect of oxygen deprivation on cell cycle activity: a profile of delay and arrest. J. Appl. Physiol., 94, 2068-2083.

Fielenbach, N., and Antebi, A. 2008. C. elegans dauer formation and the molecular basis of plasticity. Genes Dev., 22, 2149-2165.

Franke, T.F., Hornik, C.P., Segev, L., Shostak, G.A., and Sugimoto, C. 2003. PI3K/Akt and apoptosis: size matters. Oncogene, 22, 8983-8998. 
Guardavaccaro, D., and Pagano, M. 2006. Stabilizers and destabilizers controlling cell cycle oscillators. Mol. Cell., 22, 1-4.

Hand, S.C. 1998. Quiescence in Artemia franciscana embryos: reversible arrest of metabolism and gene expression at low oxygen levels. J. Exp. Biol., 201, 12331242.

Harbour, J.W., Luo, R.X., Santi, A.D., Postigo, A.A., and Dean, D.C. 1999. Cdk phosphorylation triggers sequential intramolecular interactions that progressively block $\mathrm{Rb}$ functions as cells move through G1. Cell, 98, 859-869.

Harrisingh, M.C., Perez-Nadales, E., Parkinson, D.B., Malcolm, D.S., Mudge, A.W., and Lloyd, A.C. 2004. The Ras/Raf/ERK signaling pathway drives Schwann cell differentiation. EMBO J., 23, 3061-3071.

Kitagawa, M., Higashi, H., Suzuki-Takahashi, I., Segawa, K., Hanks, S.K., Taya, Y., Nishimura, S., Okuyama, A. 1995. Phosphoylation of E2F-1 by cyclinA-cdk2. Oncogene, 10(2), 229-236.

Johnson, D., Walker, C. 1999. Cyclins and cell cycle checkpoints. Ann. Rev. Pharmacol. Toxicol., 39, 295-312.

Kelly, D.A., and Storey, K.B. 1988. Organ-specific control of glycolysis in the anoxic turtle. Am. J. Physiol. Regu. Physiol., 225(5), R774-R779.

Koff, A., Giordano, A., Desai, D., Yamashita, K., Harper, J., and Elledge, S. 1992. Formation and activation of a cyclin E-cdk2 complex during the $\mathrm{G}_{1}$ phase of the human cell cycle. Science, 257, 1689-1694.

Kops, G., Medema, R., Glassford, J., Essers, M., Dijkers, P., Coffer, P., Lam, E., and Burgering, B. 2002. Control of cell cycle exit and entry by protein kinase Bregulated forkhead transcription factors. Mol. Cell. Biol., 22, 2025-2036.

Lant, B., and Storey, K.B. 2010. An overview of stress response and hypometabolic strategies in Caenorhabditis elegans: conserved and contrasting signals with the mammalian system. Int. J. Biol. Sci., 6(1), 9-50.

Lipton, P. 1999. Ischemic cell death in brain neurons. Physiol. Rev., 17, 1431-1568.

Lin, S., Wang, W., Wilson, G.M., Yang, X., Brewer, G., Holbrook, N.J., and Gorospe, M. 2000. Down-regulation of cyclin D1 expression by prostaglandin $A_{2}$ is mediated by enhanced cyclin D1 mRNA turnover. Mol. Cell. Biol., 20(21), 7903-7913.

Lindqvist, A., van Zon, W., Rosenthal, C.K., Wolthuis, R.M.F. 2007. Cyclin B-Cdk1 activation continues after centrosome separation to control mitotic progression. PLoS Biol., 5(5), e123.

Liu, Q., Fu, H., Sun, F., Zhang, H., Tie, Y., Zhu, J., Xing, R., Sun, Z., and Zheng, X. 2008. MiR-16 family induces cell cycle arrest by regulating multiple cell cycle genes. Nucleic Acids Res., 36, 5391-5404.

Long, J., Leresche, A., Kriwacki, R., and Gottesfeld, J. 1998. Repression of TFIIH transcriptional activity and TFIIH-associated cdk7 kinase activity at mitosis. Mol. Cell Biol., 18, 1467-1476. 
Lundberg, A., and Weinberg, R. 1999. Control of the cell cycle and apoptosis. Eur. J. Cancer, 35, 1886-1894.

Maller, J. 1991. Mitotic control. Curr. Opin. Cell Biol., 3, 269-275.

Matsushime, H., Quelle, D., Shurtleff, S., Shibuya, M., Sherr, C., and Kato, J. 1994. Dtype cyclin-dependent kinase activity in mammalian cells. Mol. Cell Biol., 14, 2066-2076.

Nurse, P. 1990. Universal control mechanism regulating cell cycle timing of M-phase. Nature, 344, 503-508.

Obaya, A., and Sedivy, J. 2002. Regulation of cyclin-cdk activity in mammalian cells. Cell Mol. Life Sci., 59, 126-142.

Ortega, S., Malumbres, M., and Barbacid, M. 2002. Cell cycle and cancer: The G1 restriction point and the G1/S transition. Curr. Genom., 3, 245-263.

Padilla, P.A., Nystul, T.G., Zager, R.A., Johnson, A.C., and Roth, M.B. 2002. Dephosphorylation of cell cycle-regulated proteins correlates with anoxia-induced suspended animation in Caenorhabditis elegans. Mol. Biol. Cell., 13, 1473-1483.

Padilla, P.A., and Roth, M.B. 2001. Oxygen deprivation causes suspended animation in the zebrafish embryo. Proc. Natl. Acad. Sci. USA, 98, 7331-7335.

Papst, P., Sugiyama, H., Nagasawa, M., Lucas, J., Maller, J., and Terada, N. 1998. Cdc2cyclin B phosphorylates p70 S6 kinase on Ser411 at mitosis. J. Biol. Chem., 273, 15077-15084.

Pines, J., Hunter, T. 1989. Isolation of a human cyclin cDNA: evidence for a cyclin mRNA and protein regulation in the cell cycle and for interaction with p34. Cell, $58,833-846$.

Storey, K.B., and Hochachka, P.W. 1974. Enzymes of energy metabolism in a vertebrate facultative anaerobe, Pseudemys scripta: turtle heart phosphofructokinase. J. Biol. Chem. 249, 1417-1422.

Takuwa, N., Fukui, Y., and Takuwa, Y. 1999. Cyclin D1 expression mediated by phosphatidylinositol 3-kinase through mTOR-p70S6K-independent signaling in growth factor-stimulated NIH 3 T3 fibroblasts. Mol. Cell. Biol., 19, 1346-1358.

Tian, R.Q., Wang, X.H., Hou, L.J., Jia, W.H., Yang, Q., Li, Y.X., Liu, M., Li, X., and Tang, H. 2011. MicroRNA-372 is down-regulated and targets cyclin-dependent kinase 2 (CDK2) and Cyclin A1 in hHuman cervical cancer, which may contribute to tumorigenesis. J. Biol. Chem., 286(29), 25556-25563.

Wang, W., Caldwell, M.C., Lin, S., Furneaux, H., and Gorospe, M. 2000. HuR regulates cyclin A and cyclin B mRNA stability during cell proliferation. EMBO J., 19, 2340-2350.

Weinberg, R. 1995. The retinoblastoma protein and cell cycle control. Cell., 81, 323-330.

Weinmann, A.S., Bartley, S.M., Zhang, M.Q., and Farnham, P.J. 2001. Use of chromatin immunoprecipitation to clone novel E2F target promoters. Mol. Cell. Biol., 21(20), 6820-6832. 
Wiestner, A., Tehrani, M., Chiorazzi, M., Wright, G., Gibellini, F., Nakayama, K., Liu, H., Rosenwald, A., Muller-Hermelink, H.K., Ott, G., Chan, W.C., Greiner, T.C., Weisenburger, D.D., Vose, J., Armitage, J.O., Gascoyne, R.D., Connors, J.M., Campo, E., Montserrat, E., Bosch, F., Smeland, E.B., Kvaloy, S., Holte, H., Delabie, J., Fisher, R.I., Grogan, T.M., Miller, T.P., Wilson, W.H., Jaffe, E.S., Staudt, L.M. 2007. Point mutations and genomic deletions in CCND1 create stable truncated cyclin D1 mRNAs that are associated with increased proliferation rate and shorter survival. Blood, 109, 4599-606.

Wilson, R.E., Keng, P.C., and Sutherland, R.M. 1990. Changes in growth characteristics and macromolecular synthesis on recovery from severe hypoxia. Br. J. Cancer, 61, 14-21.

Yang, K., Guo, Y., Stacey, W.C., Harwalkar, J., Fretthold, J., Hitomi, M., and Stacey, D.W. 2006. Glycogen synthase kinase 3 has a limited role in cell cycle regulation of Cyclin D1 levels. BMC Cell. Biol., 7, 33.

Zhang, J., Biggar, K.K., and Storey, K.B. 2013. Regulation of p53 by reversible posttranscriptional and post-translational mechanisms in liver and skeletal muscle of an anoxia tolerant turtle, Trachemys scripta elegans. Gene, 513(1), 147-155. 


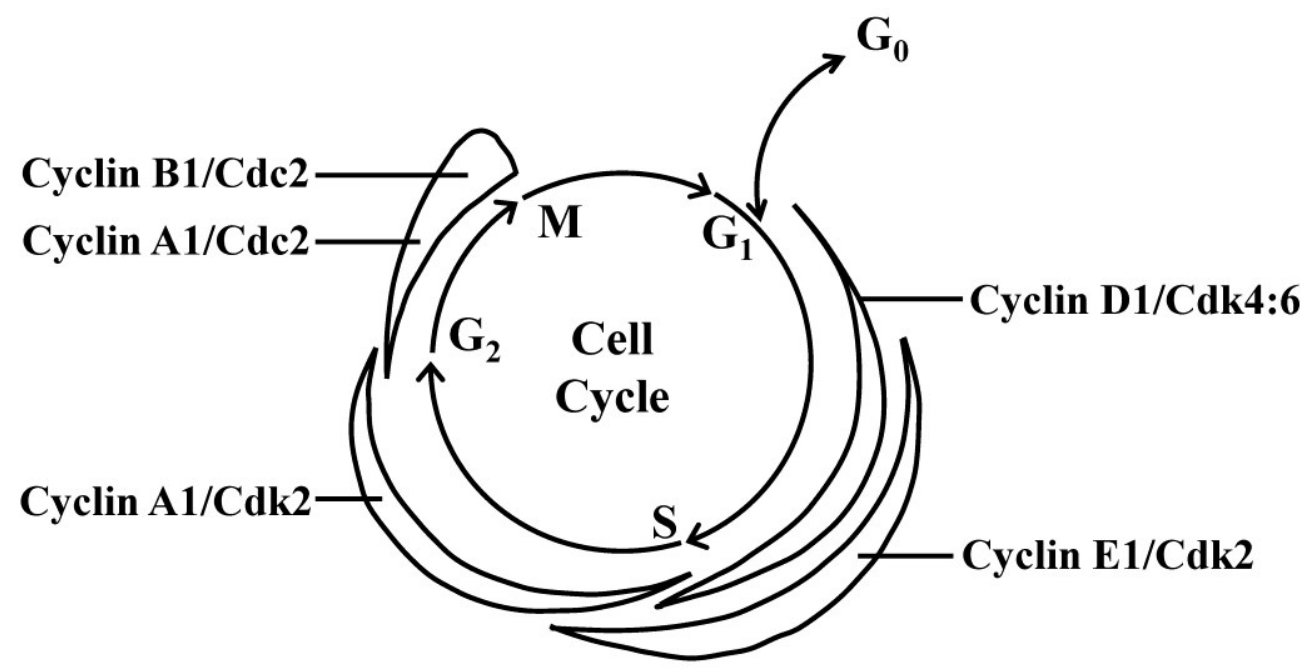

Figure 2.1. Expression profiles of Cyclin/Cdk complexes throughout the cell cycle. Cyclic expression of these complexes allow for the completion of one phase before the initiation of the subsequent phase of the cell cycle. 


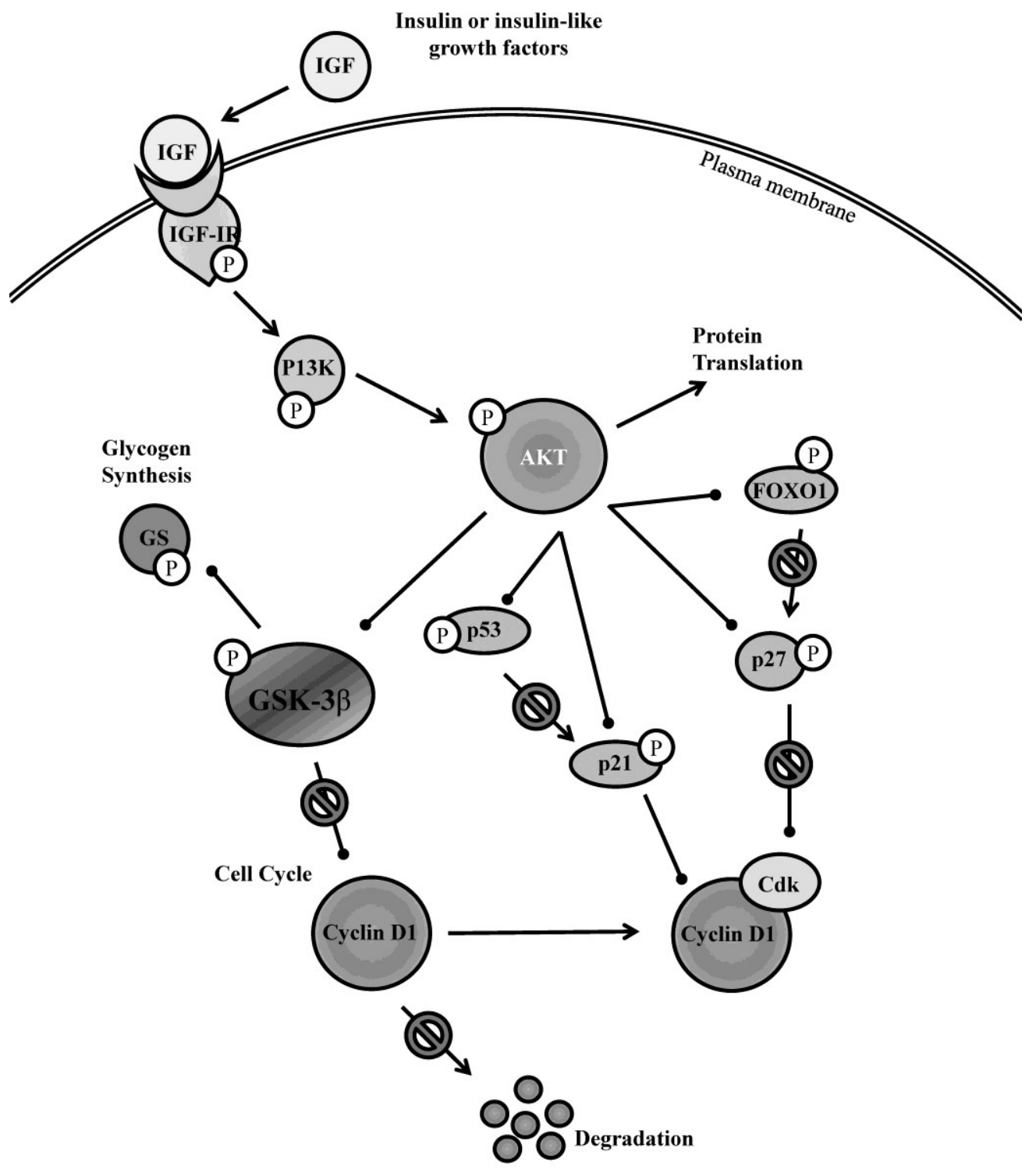

Figure 2.2. Regulation of Cyclin D1 degradation by Akt/PI3K signaling and regulation of GSK-3 $\beta$ activity. 


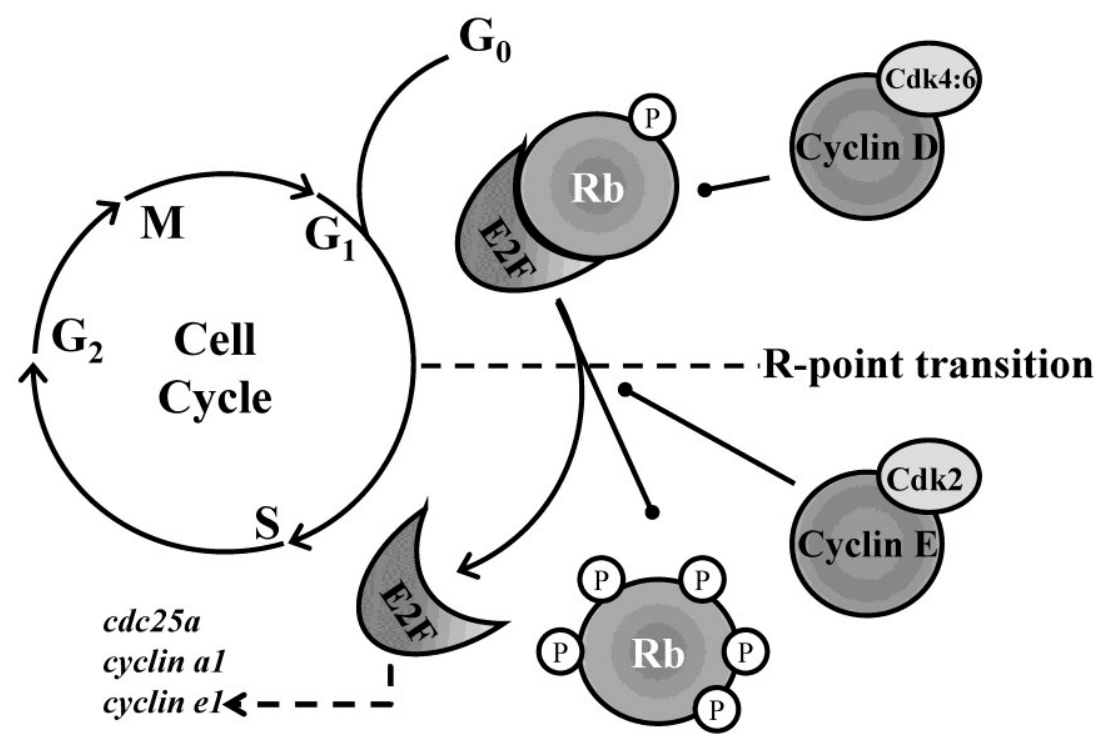

Figure 2.3. The Rb/E2F pathway. Sequential phosphorylation by kinase complexes Cyclin D1/Cdk4:Cdk6 and Cyclin E1/Cdk2, respectively, causes conformational changes to the $\mathrm{Rb}$ structure and release of E2F. The release of E2F is necessary for the expression of S-phases genes. 

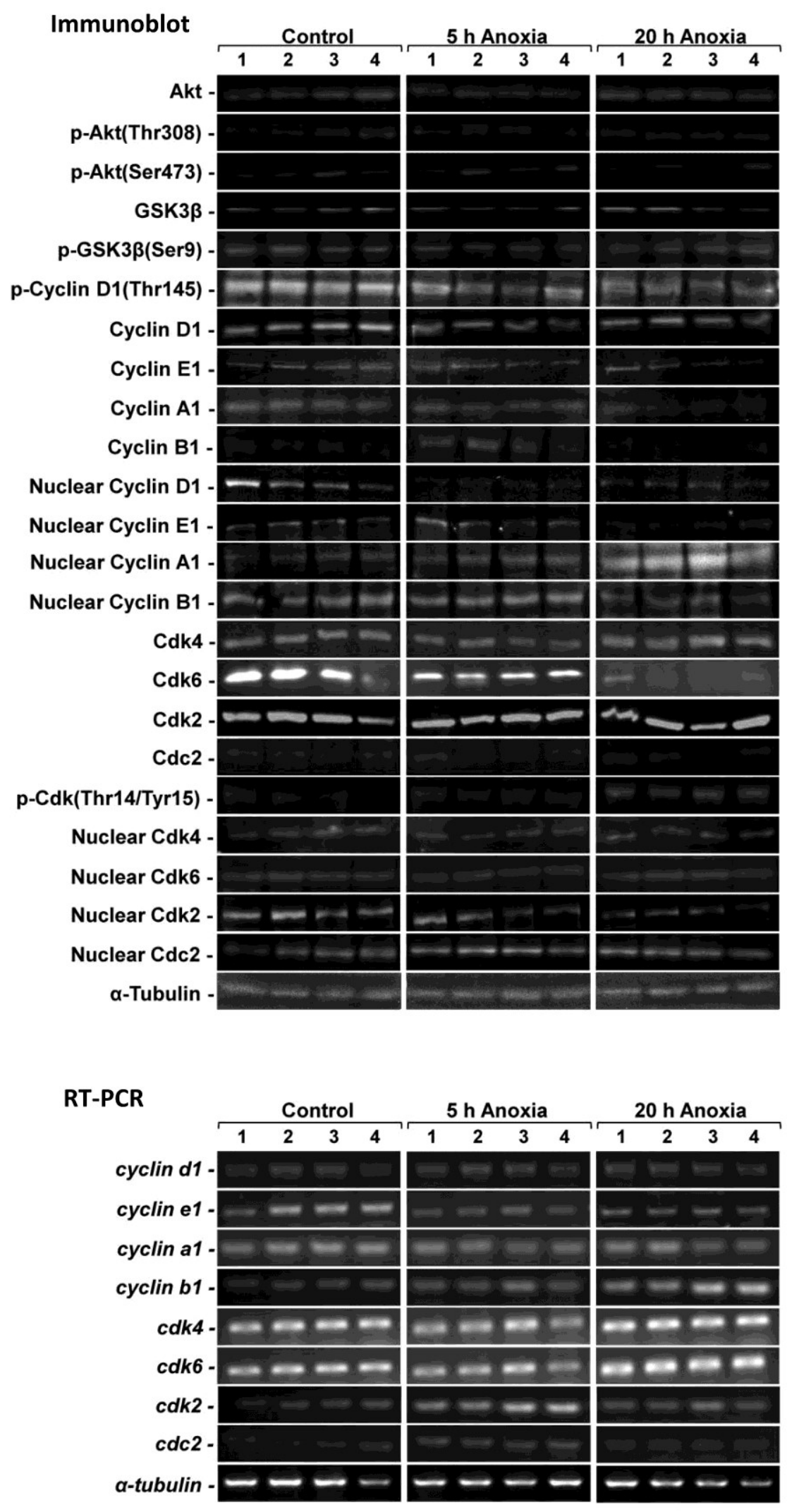

Figure 2.4A. Image files for immunoblotting and RT-PCR targets in control and anoxic (5 and $20 \mathrm{~h}$ ) liver tissue from T. scripta elegans. 


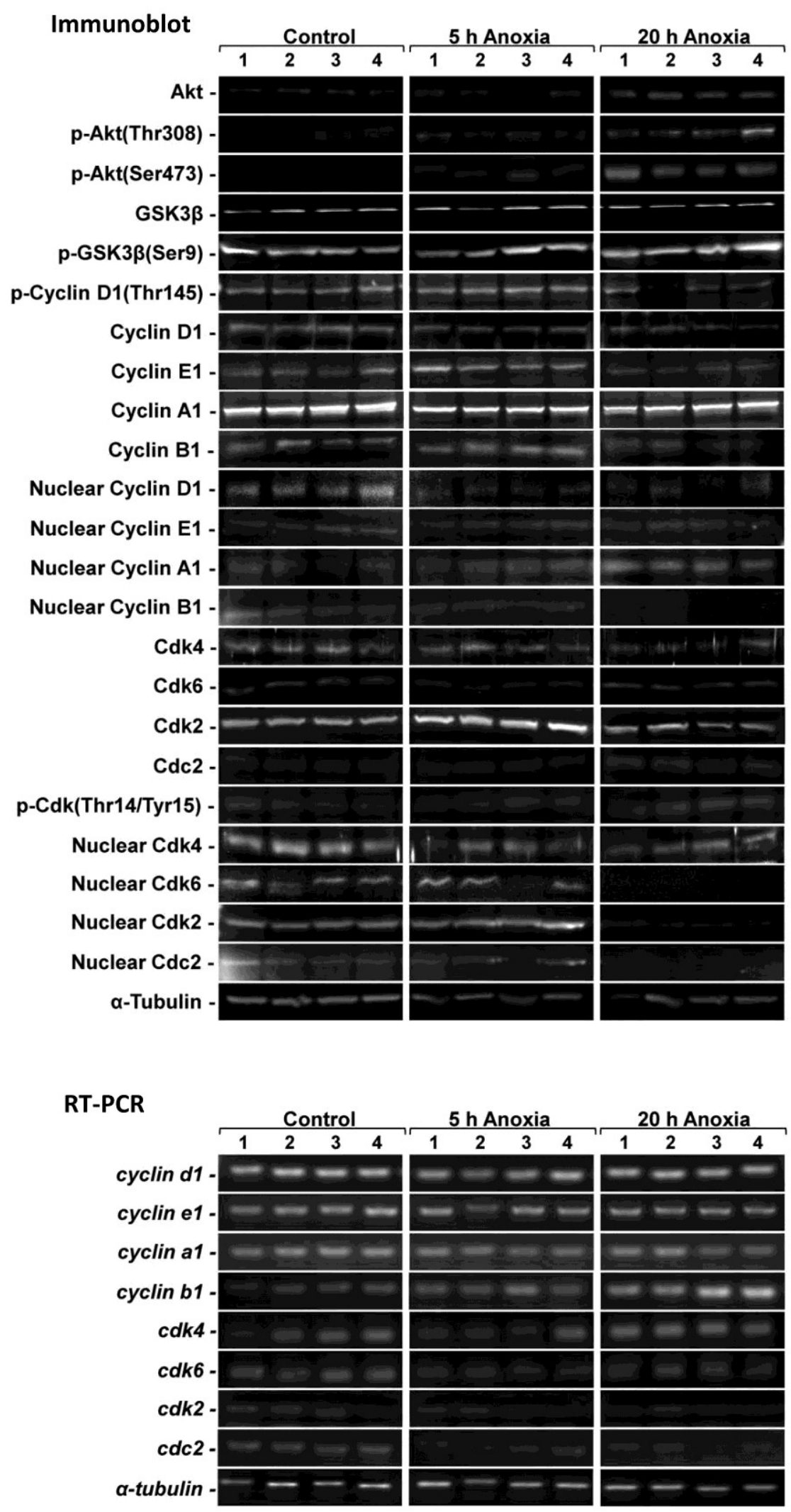

Figure 2.4B. Image files for immunoblotting and RT-PCR targets in control and anoxic (5 and 20 h) kidney tissue from T. scripta elegans. 

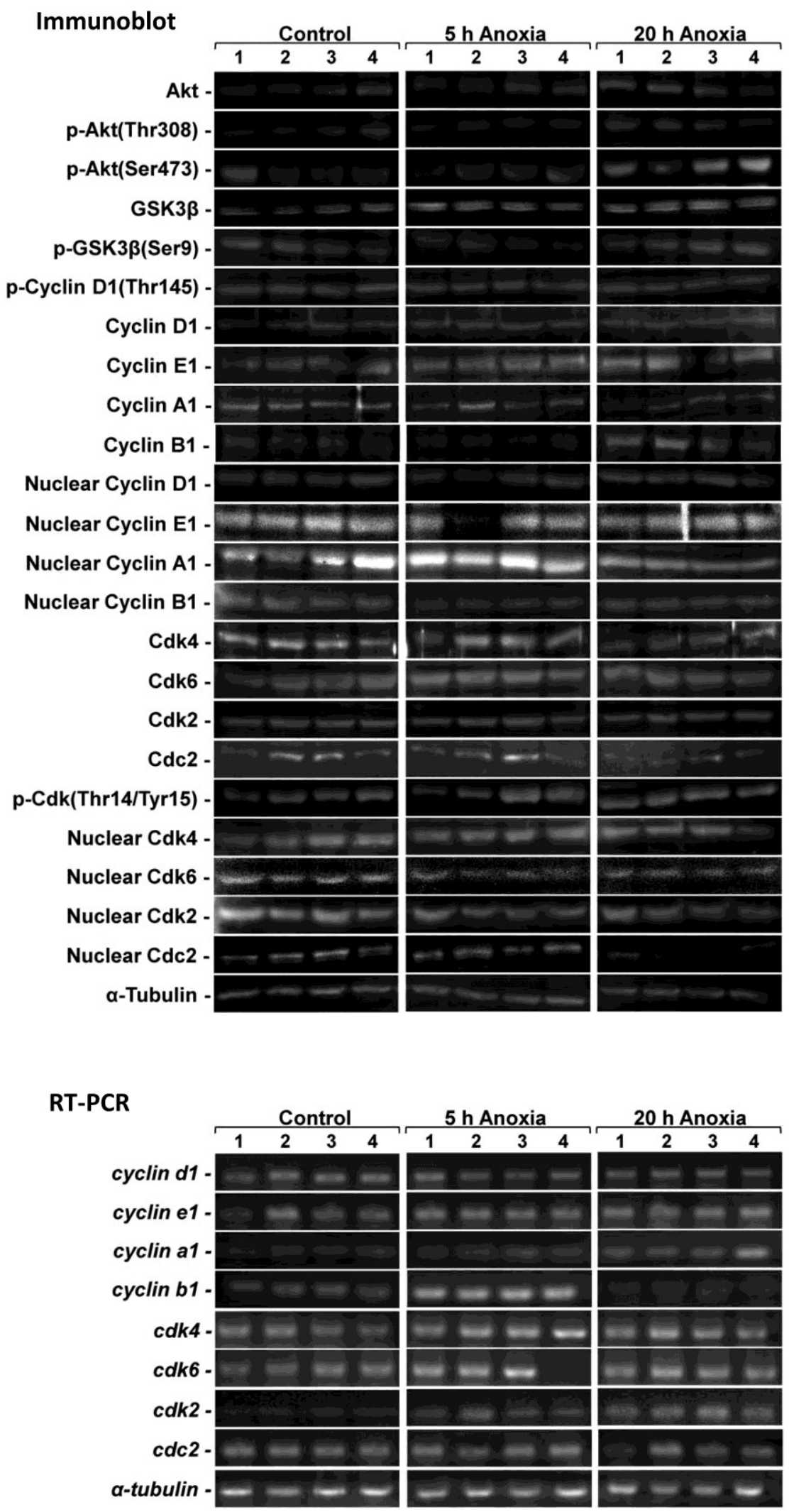

Figure 2.4C. Image files for immunoblotting and RT-PCR targets in control and anoxic (5 and $20 \mathrm{~h}$ ) white skeletal muscle tissue from T. scripta elegans. 

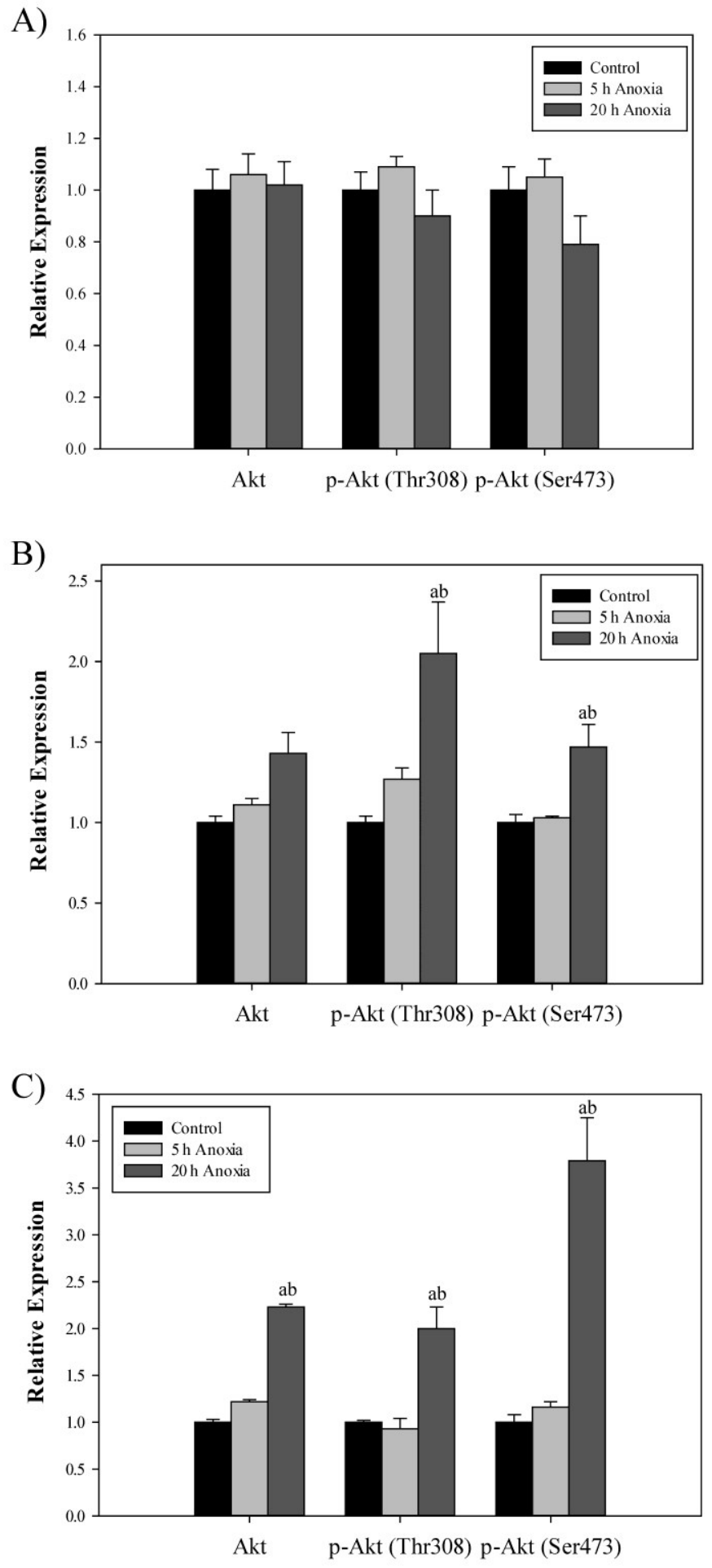

Figure 2.5. Effect of 5 and $20 \mathrm{~h}$ of anoxic submergence on relative protein and phosphorylation levels of Akt in T. s. elegans. Histograms show normalized expression levels under control and anoxic conditions in (A) liver, (B) kidney and (C) white skeletal muscle tissues. Data are means \pm S.E.M., $n=3-4$ independent trials. a: Significantly different from the corresponding control $(\mathrm{P}<0.05)$; b: significantly different from the $5 \mathrm{~h}$ anoxic value $(\mathrm{P}<0.05)$. 

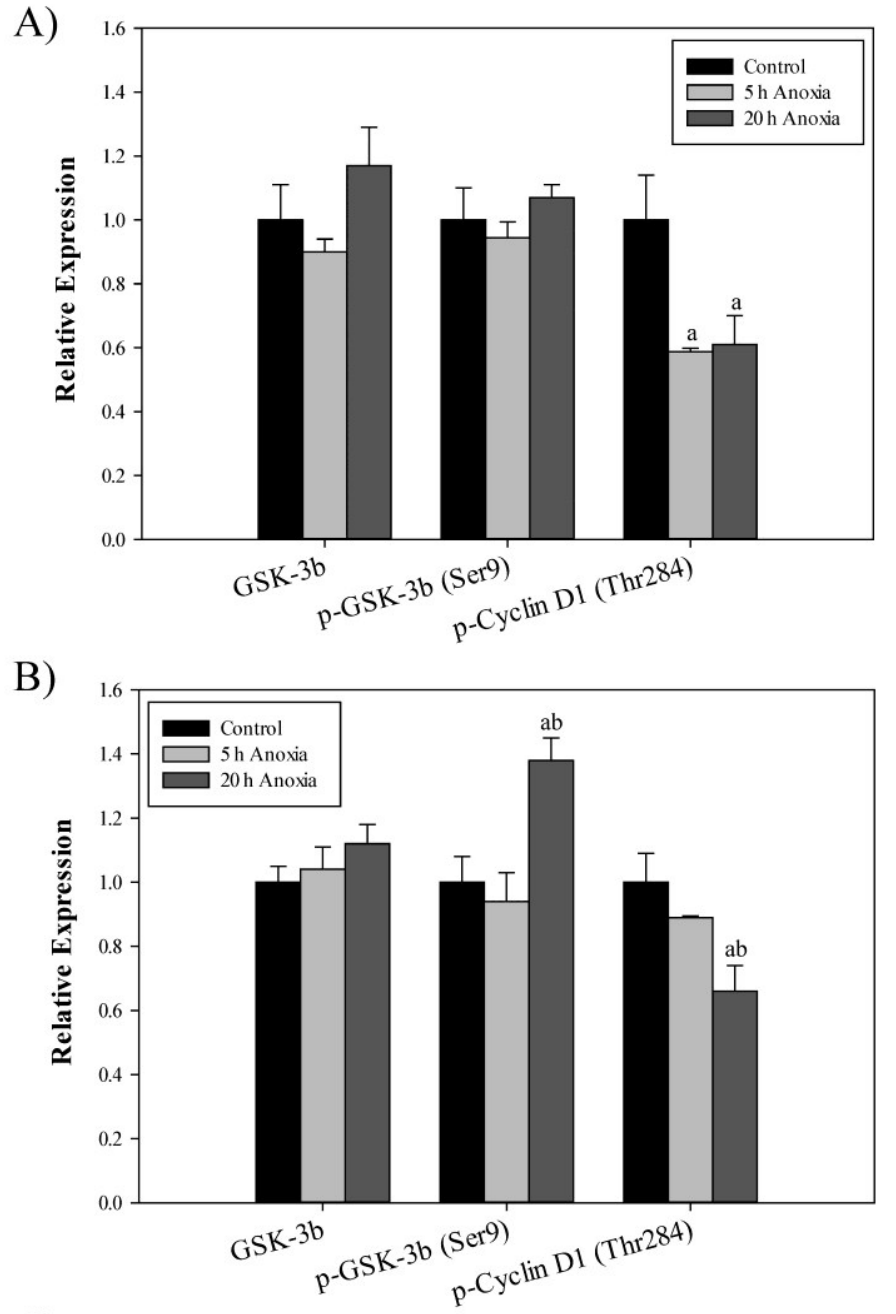

C)

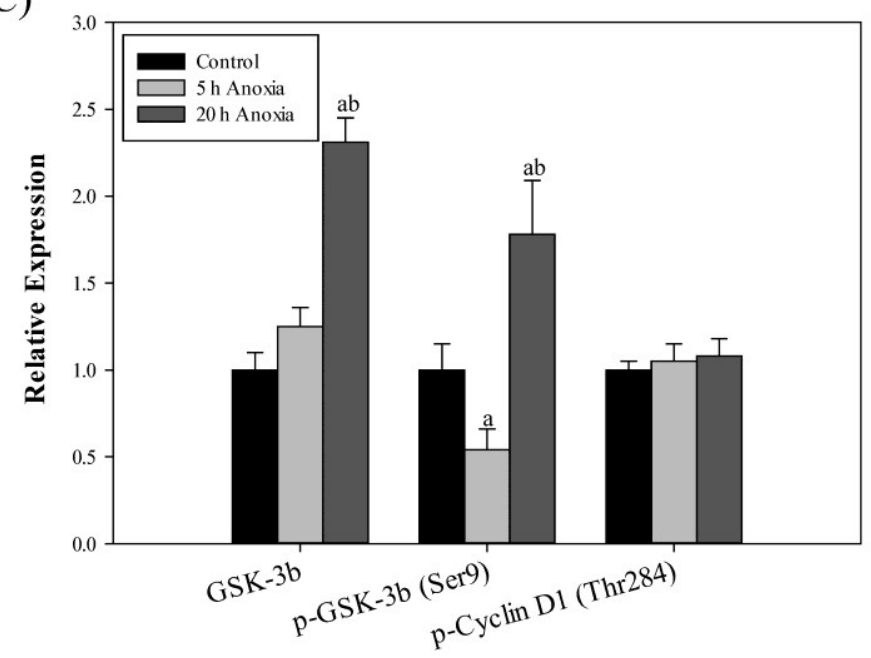

Figure 2.6. Effect of 5 and $20 \mathrm{~h}$ of anoxic submergence on relative protein and phosphorylation levels of GSK-3b and phosphorylation of its downstream target, Cyclin D1 in T. s. elegans. Histograms show normalized expression levels under control and anoxic conditions in (A) liver, (B) kidney and (C) white skeletal muscle tissues. Data are means \pm S.E.M., $n=3-4$ independent trials. a: Significantly different from the corresponding control $(\mathrm{P}<0.05)$; b: significantly different from the $5 \mathrm{~h}$ anoxic value $(\mathrm{P}<0.05)$. 
A)

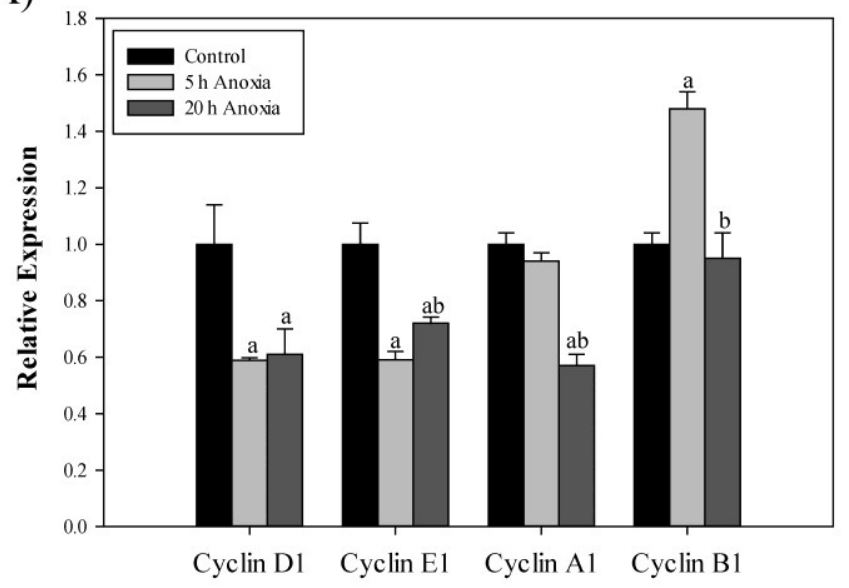

B)

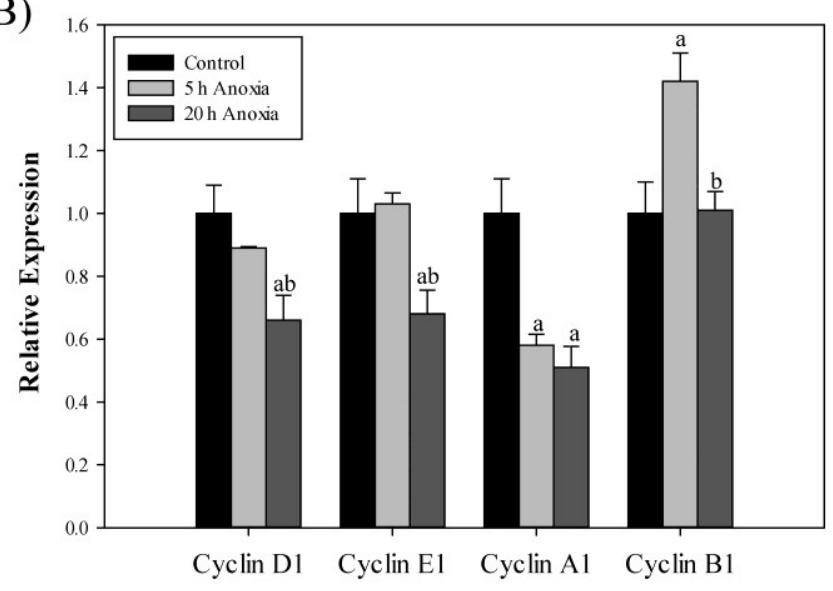

C)

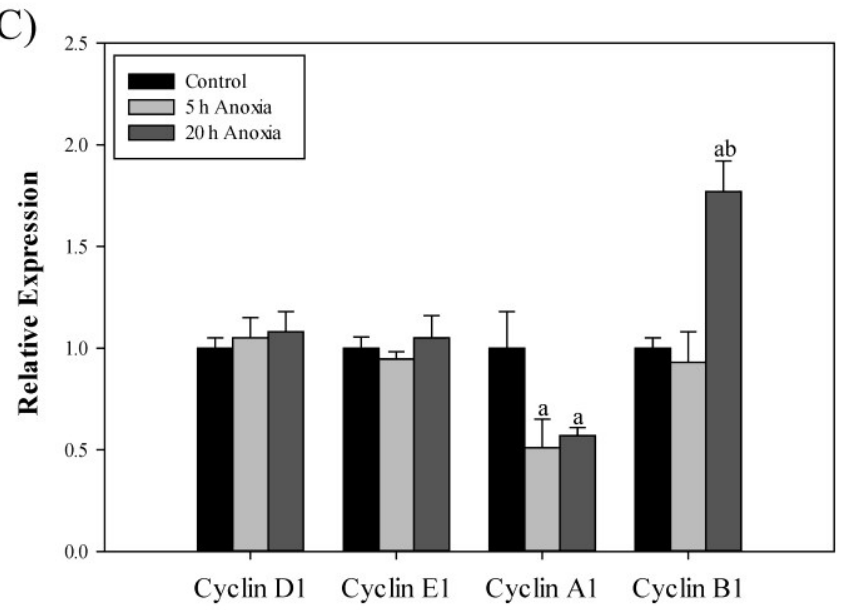

Figure 2.7. Effect of 5 and $20 \mathrm{~h}$ of anoxic submergence on relative protein levels of Cyclins (types D1, E1, A1 and B1) in T. s. elegans. Histograms show normalized expression levels under control and anoxic conditions in (A) liver, (B) kidney and (C) white skeletal muscle tissues. Data are means \pm S.E.M., $\mathrm{n}=3$ 4 independent trials. a: Significantly different from the corresponding control $(\mathrm{P}<0.05)$; b: significantly different from the $5 \mathrm{~h}$ anoxic value $(\mathrm{P}<0.05)$. 


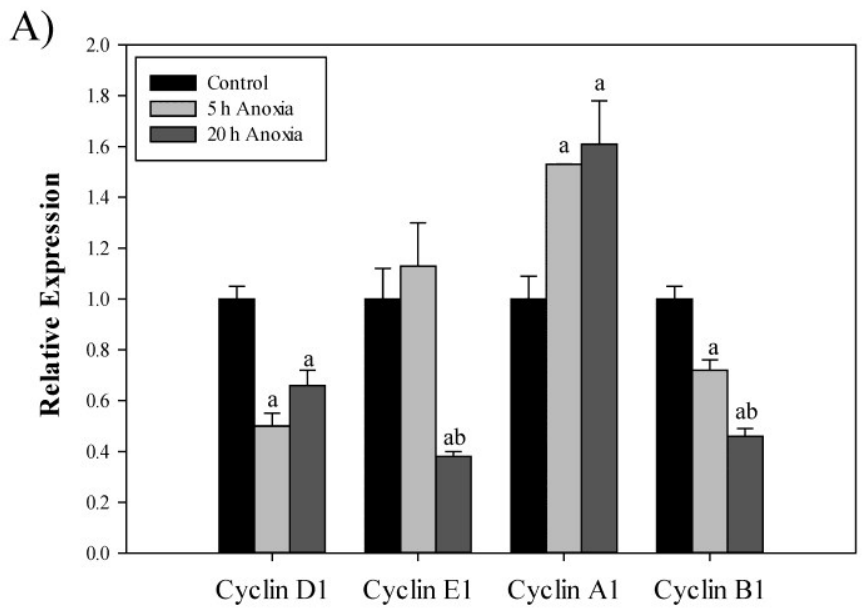

B)
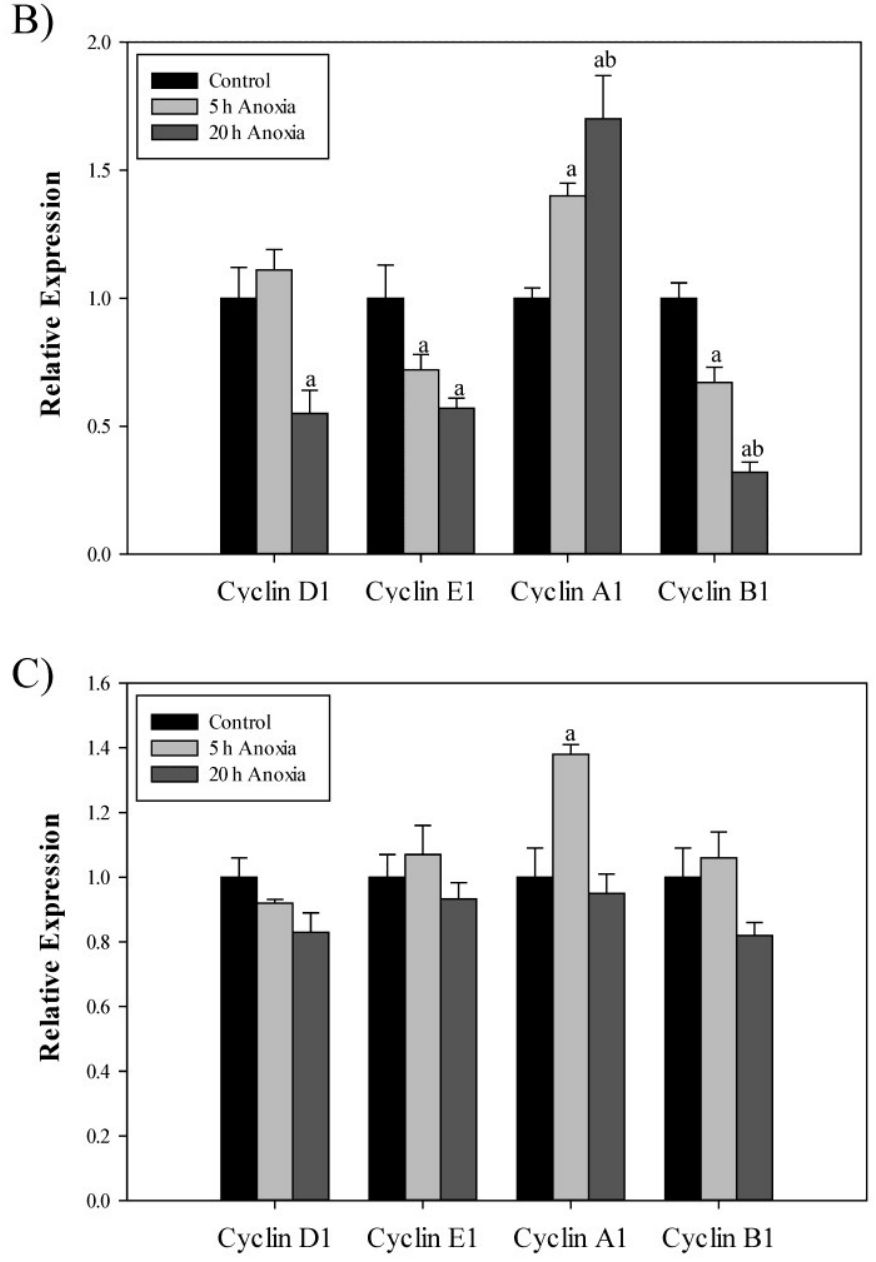

Figure 2.8. Effect of 5 and $20 \mathrm{~h}$ of anoxic submergence on relative nuclear protein levels of Cyclins (types D1, E1, A1 and B1) in T. s. elegans. Histograms show normalized expression levels under control and anoxic conditions in nuclear fractions from (A) liver, (B) kidney and (C) white skeletal muscle tissues. Data are means \pm S.E.M., n= 3-4 independent trials. a: Significantly different from the corresponding control $(\mathrm{P}<0.05)$; b: significantly different from the $5 \mathrm{~h}$ anoxic value $(\mathrm{P}<0.05)$. 
A)

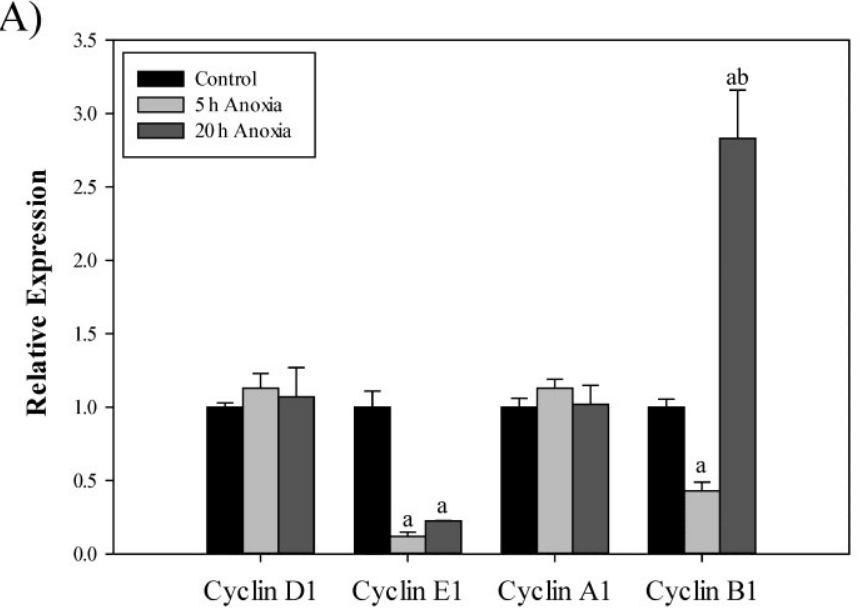

B)

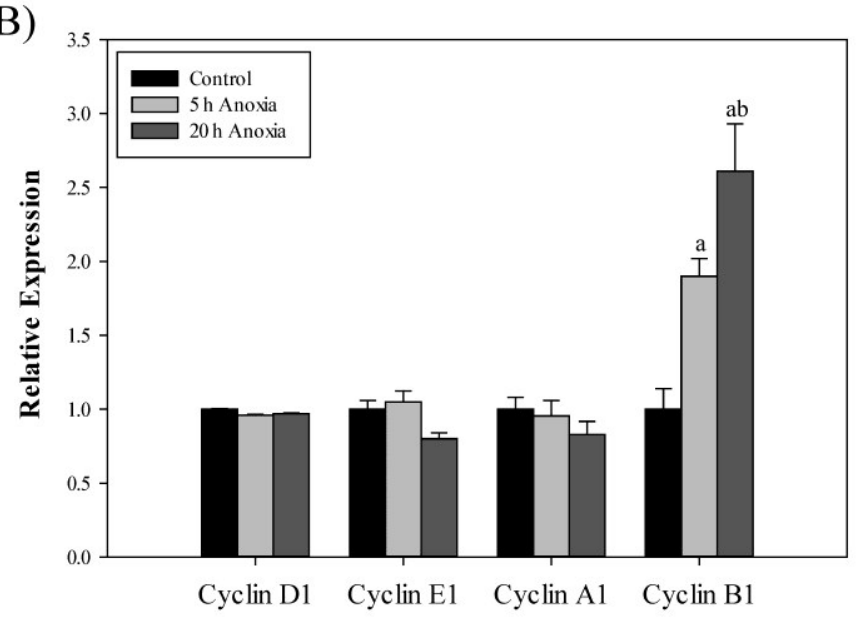

C)

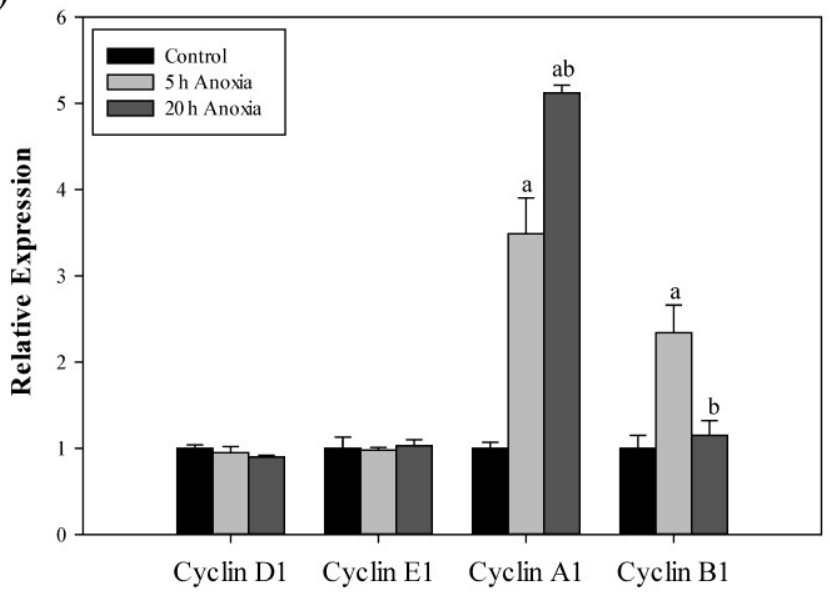

Figure 2.9. Effect of 5 and $20 \mathrm{~h}$ of anoxic submergence on transcript levels of cyclins (types $d 1, e 1, a 1$ and $b l$ ) in T. s. elegans. Histograms show normalized expression levels under control and anoxic conditions in (A) liver, (B) kidney and (C) white skeletal muscle tissues. Data are means \pm S.E.M., $n=3-$ 4 independent trials. a: Significantly different from the corresponding control $(\mathrm{P}<0.05)$; b: significantly different from the $5 \mathrm{~h}$ anoxic value $(\mathrm{P}<0.05)$. 
A)

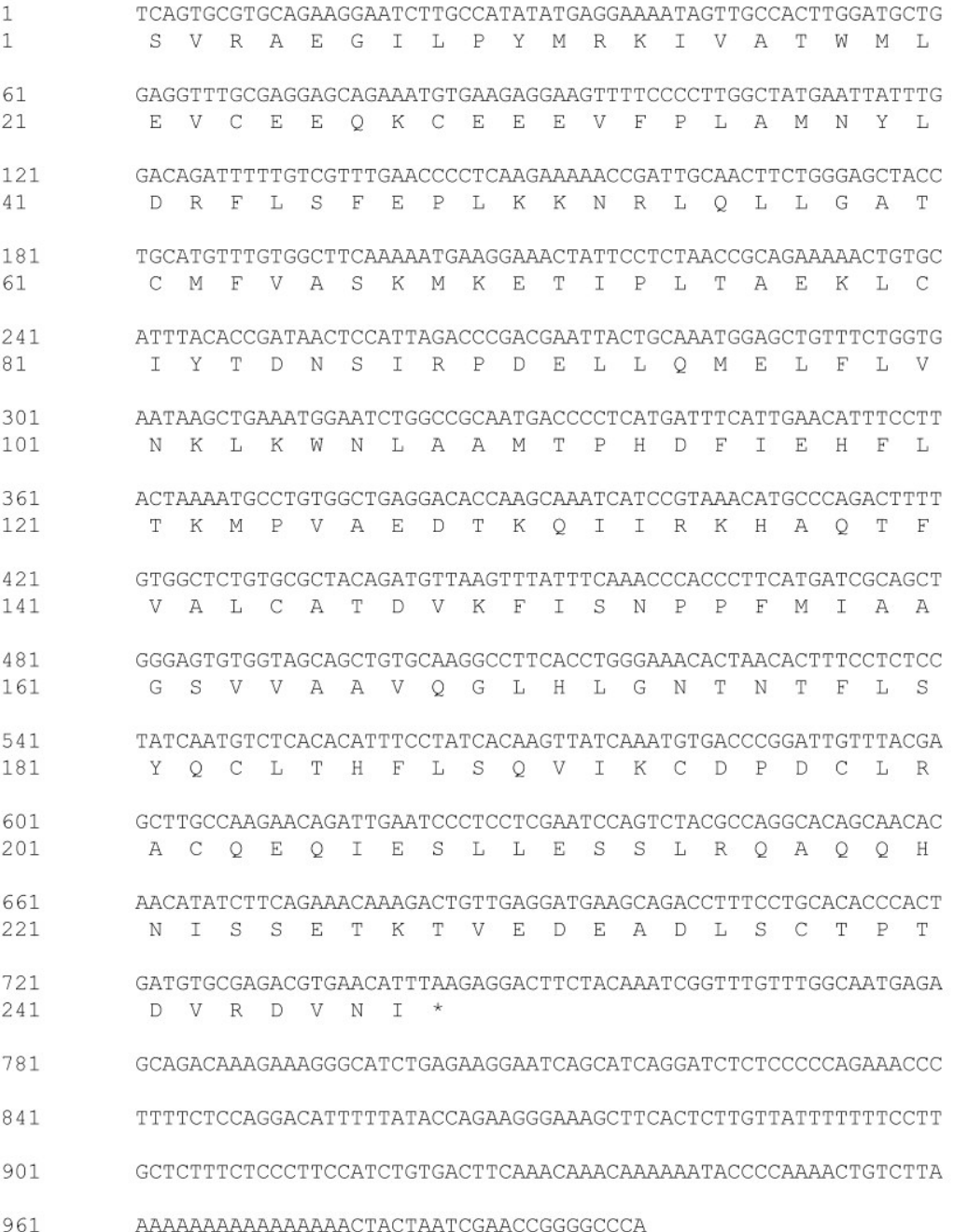

B)

C)

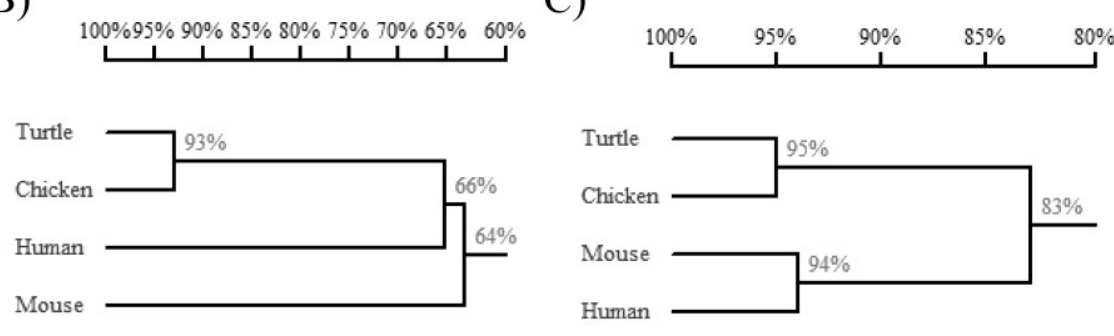

Figure 2.10. Nucleotide and deduced amino acid sequence for T. s. elegans partial cyclin $d l$ sequence. (A) Nucleotides and amino acids are numbered on the left. The nucleotide sequence was 998 nucleotides in length, while the amino acid sequence was 247 amino acids in length. Homology of (B) nucleotide and (C) protein sequences of the turtle to that of chicken (G. gallus), Mouse ( $M$. musculus) and human (H. sapiens). 
A)

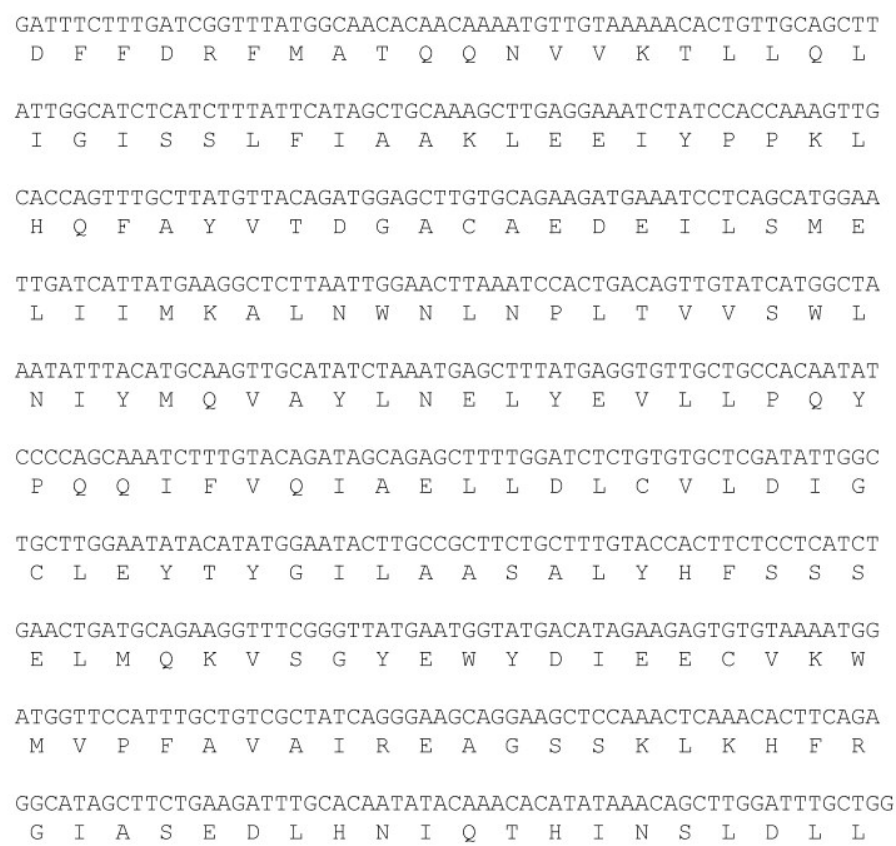

B)
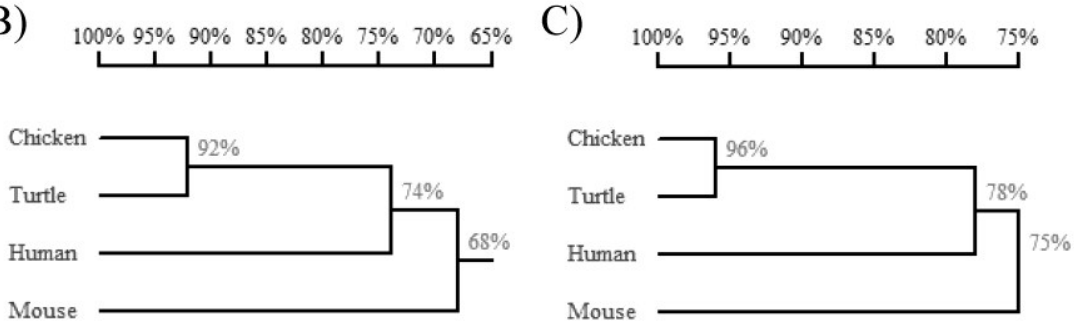

Figure 2.11. Nucleotide and deduced amino acid sequence for T. s. elegans partial cyclin e1 sequence. (A) Nucleotides and amino acids are numbered on the left. The nucleotide sequence was 601 nucleotides in length, while the amino acid sequence was 190 amino acids in length. Homology of (B) nucleotide and (C) protein sequences of the turtle to that of chicken (G. gallus), Mouse (M. musculus) and human (H. sapiens). 
A)

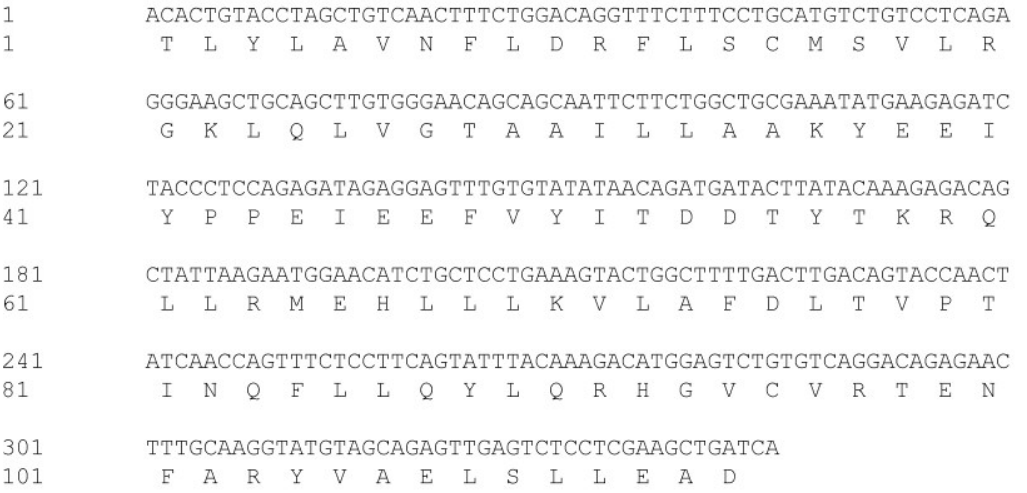

B)
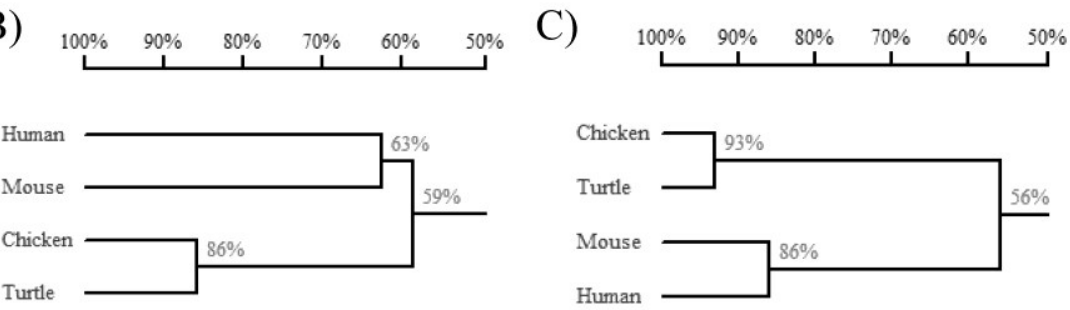

Figure 2.12. Nucleotide and deduced amino acid sequence for $T$. s. elegans partial cyclin al sequence. (A) Nucleotides and amino acids are numbered on the left. The nucleotide sequence was 345 nucleotides in length, while the amino acid sequence was 114 amino acids in length. Homology of (B) nucleotide and (C) protein sequences of the turtle to that of chicken (G. gallus), Mouse ( $M$. musculus) and human (H. sapiens). 
A)

GTGTGCCCAAGAAGATGTTGCAGCTGGTTGGCGTCACGGCCATGTTTATTGCCAGCAAGT

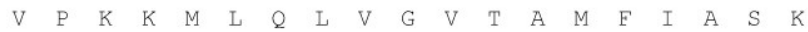

ATGAGGAAATGTATCCTCCTGAAATTGGGGACTTTGCCTTTGTAACAGACCACACTTACA

$\begin{array}{llllllllllllllllllll}Y & E & E & M & Y & P & P & E & I & G & D & F & A & F & V & T & D & H & T & Y\end{array}$

CCAAGTTCCAGATCAAACAAATGGAAAGGAAGATTCTACAAGCTCTAGACTTTGCCTTGG

$\begin{array}{lllllllllllllllllllll}T & K & F & Q & I & K & Q & M & E & R & K & I & L & Q & A & L & D & F & A & L\end{array}$

GCCGCCCTCTACCACTGCACTTTCTAAGGAGGGCATCAAAGATTGGAGAGGTGGACCTAG

$\begin{array}{llllllllllllllllllll}G & R & P & L & P & L & H & F & L & R & R & A & S & K & I & G & E & V & D & L\end{array}$

AACAGCACACTCTAGCCAAATATCTGATGGAATTATCCCTGGTGGATTATGAAATGGTAC

$\begin{array}{llllllllllllllllllll}E & Q & H & T & L & A & K & Y & L & M & E & L & S & L & V & D & Y & E & M & V\end{array}$

ACTACCCTCCATCCCAGATTGCTGCAGCTGCTTTTTGCTTGGCTCTAAAGGTTCTTGATG

$\begin{array}{llllllllllllllllllll}H & Y & P & P & S & Q & I & A & A & A & A & F & C & \text { L } & \text { A } & \text { L } & \text { K } & \text { V } & \text { L } & \text { D }\end{array}$

GTGGAGAGTGGACACCAACTCTACAACACTACATGTCTTACACTGAGAGTGCTCTTCACC

$\begin{array}{llllllllllllllllllll}G & G & E & W & T & P & T & L & Q & H & Y & M & S & Y & T & E & S & A & L & H\end{array}$

CTGTCATGCAACATATGGCAAAGAATGTGATCCTAGTGAACCGGGGCCTTACAAAGCACA

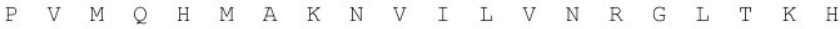

$481 \quad$ TG

$160 \quad M$

B)

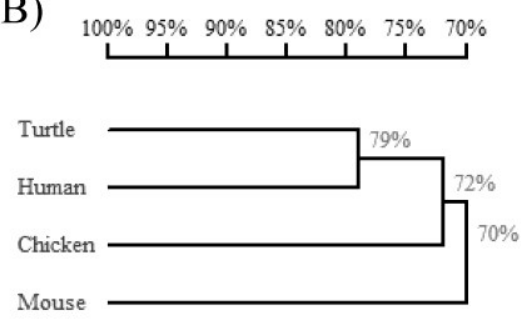

C)

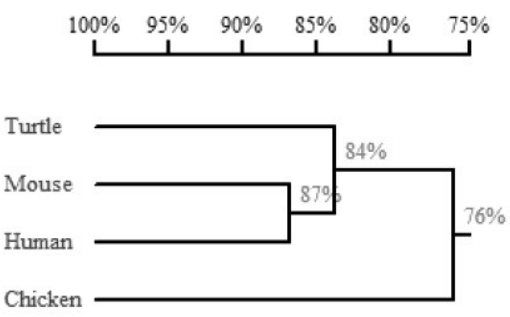

Figure 2.13. Nucleotide and deduced amino acid sequence for T. s. elegans partial cyclin b1 sequence. Nucleotides and amino acids are numbered on the left. (A) Nucleotides and amino acids are numbered on the left. The nucleotide sequence was 482 nucleotides in length, while the amino acid sequence was 160 amino acids in length. Homology of (B) nucleotide and (C) protein sequences of the turtle to that of chicken (G. gallus), Mouse (M. musculus) and human (H. sapiens). 
A)

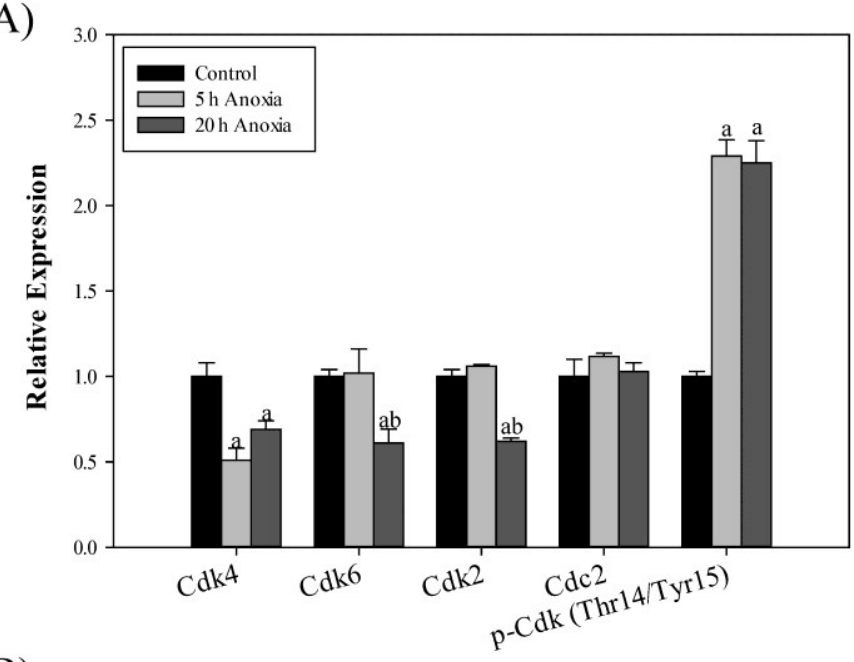

B)

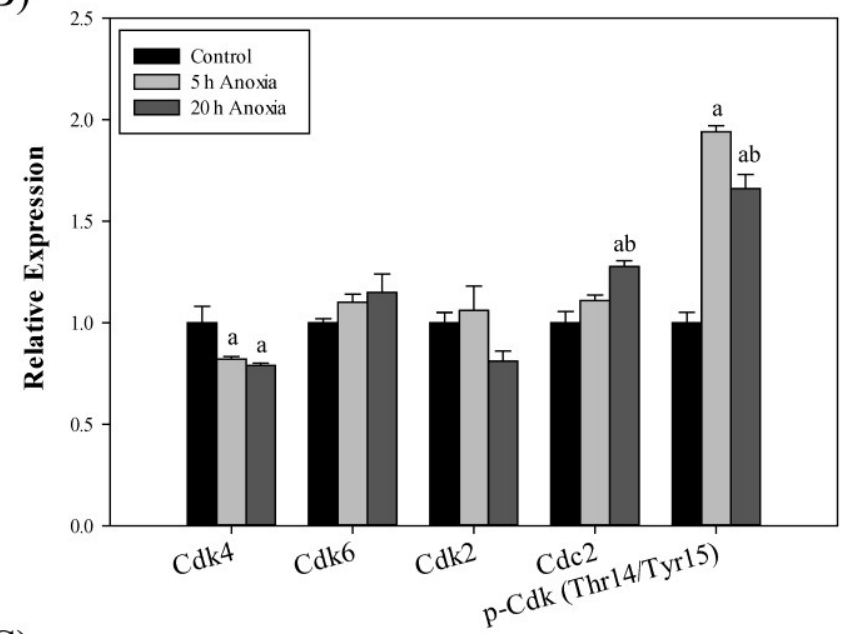

C)

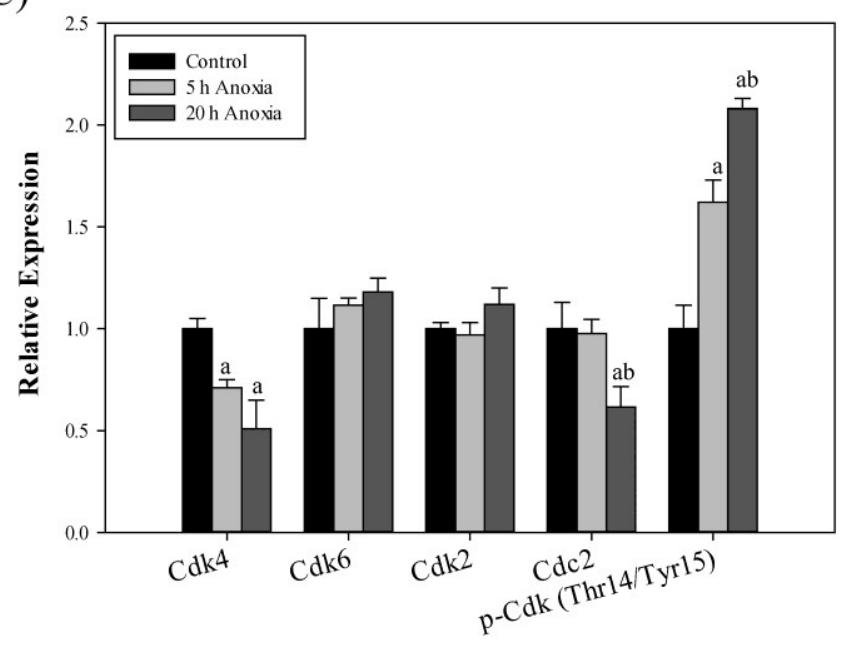

Figure 2.14. Effect of 5 and $20 \mathrm{~h}$ of anoxic submergence on relative protein and phosphorylation levels of Cdks (types 4, 6,2) and Cdc2 in T. s. elegans. Histograms show normalized expression levels under control and anoxic conditions in (A) liver, (B) kidney and (C) white skeletal muscle tissues. Data are means \pm S.E.M., $n=3-4$ independent trials. a: Significantly different from the corresponding control $(\mathrm{P}<$ $0.05)$; b: significantly different from the $5 \mathrm{~h}$ anoxic value $(\mathrm{P}<0.05)$. 

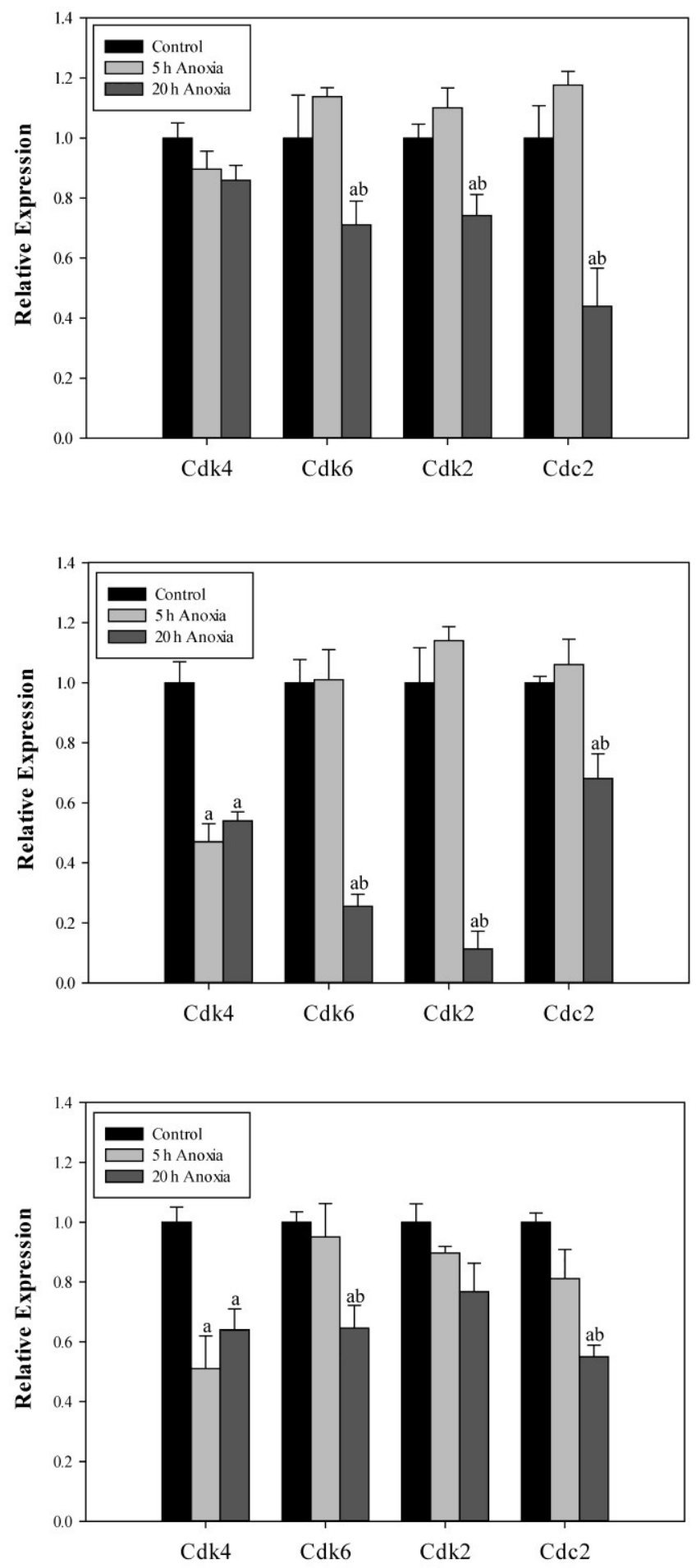

Figure 2.15. Effect of 5 and $20 \mathrm{~h}$ of anoxic submergence on relative nuclear levels of Cdks (types 4, 6, 2 ) and $\mathrm{Cdc} 2$ in T. s. elegans. Histograms show normalized expression levels under control and anoxic conditions in nuclear fractions from (A) liver, (B) kidney and (C) white skeletal muscle tissues. Data are means \pm S.E.M., $\mathrm{n}=3-4$ independent trials. a: Significantly different from the corresponding control $(\mathrm{P}<$ $0.05)$; b: significantly different from the $5 \mathrm{~h}$ anoxic value $(\mathrm{P}<0.05)$. 

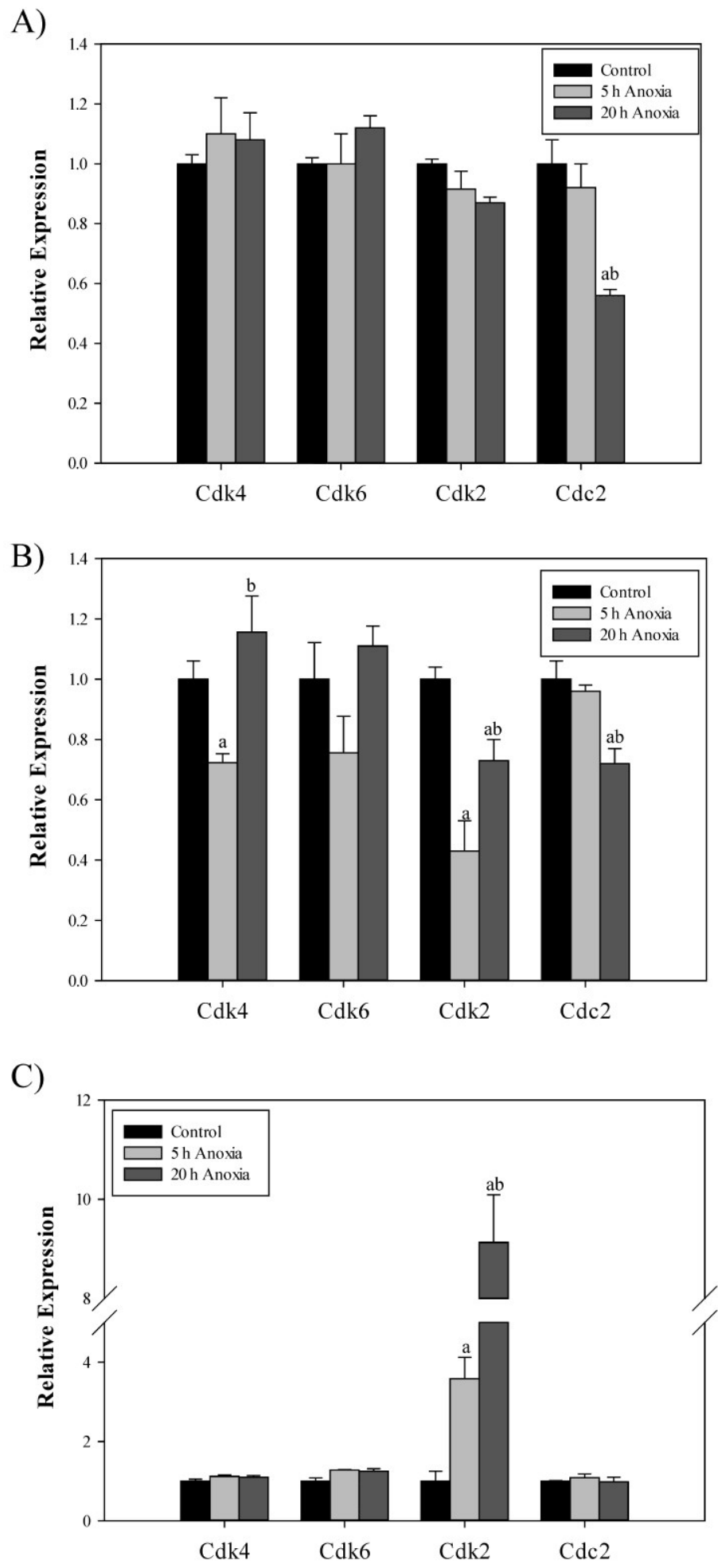

Figure 2.16. Effect of 5 and $20 \mathrm{~h}$ of anoxic submergence on transcript levels of $c d k s$ (types 4,6,2) and $c d c 2$ in T. s. elegans. Histograms show normalized expression levels under control and anoxic conditions in (A) liver, (B) kidney and (C) white skeletal muscle tissues. Data are means \pm S.E.M., $n=3-4$ independent trials. a: Significantly different from the corresponding control $(\mathrm{P}<0.05)$; b: significantly different from the $5 \mathrm{~h}$ anoxic value $(\mathrm{P}<0.05)$. 
A)

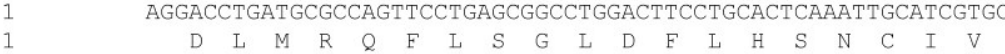

61 ACCGCGACCTGAAGCCGGAGAACATCCTCGTGACCAGCAGTGGGCAAGTGAAGCTGGCGG

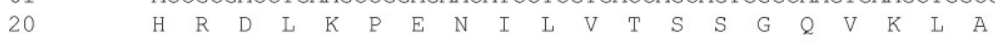

121 ACTTCGGCCTGGCCCGGATCTACAGCTGCCAGATGGCCCTGACCCCTGTGGT

$\begin{array}{llllllllllllllllllllllllll}40 & D & F & G & L & A & R & I & Y & S & C & Q & M & A & L & T & P & V\end{array}$

B)

C)
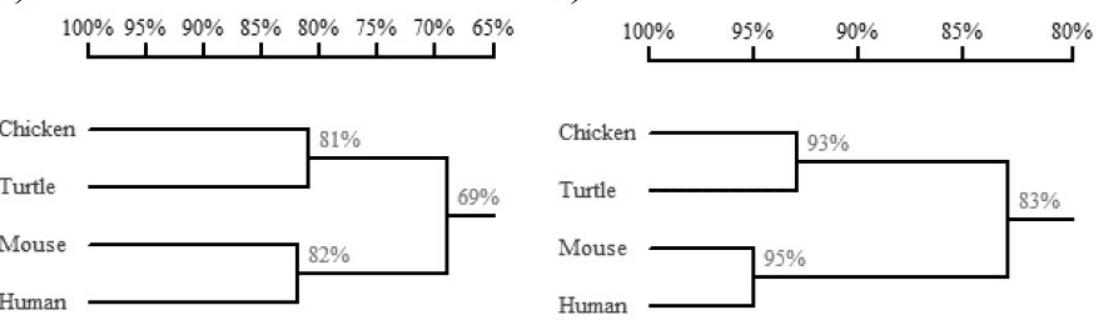

Figure 2.17. Nucleotide and deduced amino acid sequence for T. s. elegans partial $c d k 4$ sequence.

Nucleotides and amino acids are numbered on the left. (A) Nucleotides and amino acids are numbered on the left. The nucleotide sequence was 172 nucleotides in length, while the amino acid sequence was 57 amino acids in length. Homology of (B) nucleotide and (C) protein sequences of the turtle to that of chicken (G. gallus), Mouse (M. musculus) and human (H. sapiens). 
A)

GCGCAGCAGTCGATTGTAGCCTTGAGCGCGTACGGGTGCAGACAAGCGAGGAGGGAATGC

$\begin{array}{lllllllllllllllllll}A & A & V & D & C & S & L & E & R & V & R & V & Q & T & S & E & E & G & M\end{array}$

CACTCTCCACCATCCGTGAGGTGGCAGTATTGAGGCACCTGGAGACCTTTGAGCACCCCA

$\begin{array}{lllllllllllllllllllll}P & L & S & T & I & R & E & V & A & V & L & R & H & L & E & T & F & E & H & P\end{array}$

ATGTGGTCAGGTTATTTGATGTATGCACAGTGTCACGAACAGACAGAGAGACCAAACTAA

$\begin{array}{lllllllllllllllllllll}N & V & V & R & L & F & D & V & C & T & V & S & R & T & D & R & E & T & K & L\end{array}$

CACTAGTGTTTGAACATGTCGATCAAGACTTGACCACTTACTTGGATAAAGTTCCGGAGC

$\begin{array}{llllllllllllllllllll}T & \mathrm{~L} & \mathrm{~V} & \mathrm{~F} & \mathrm{E} & \mathrm{H} & \mathrm{V} & \mathrm{D} & \mathrm{Q} & \mathrm{D} & \mathrm{L} & \mathrm{T} & \mathrm{T} & \mathrm{Y} & \mathrm{L} & \mathrm{D} & \mathrm{K} & \mathrm{V} & \mathrm{P} & \mathrm{E}\end{array}$

CTGGAGTGCCTACGGAAACTATAAAGGACATGATGCTTCAGCTGTTGCGAGGGCTGGATT

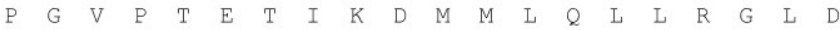

TTCTGCATTCACACCGAGTGGTGCACCGTGATCTGAAACCACAAAATATCCTCGTGACGA

$\begin{array}{llllllllllllllllllll}\mathrm{F} & \mathrm{L} & \mathrm{H} & \mathrm{S} & \mathrm{H} & \mathrm{R} & \mathrm{V} & \mathrm{V} & \mathrm{H} & \mathrm{R} & \mathrm{D} & \mathrm{L} & \mathrm{K} & \mathrm{P} & \mathrm{Q} & \mathrm{N} & \mathrm{I} & \mathrm{L} & \mathrm{V} & \mathrm{T}\end{array}$

GCAGTGGGCAGATAAAATTAGCTGACTTTGGCCTTGCACGAATCTACAGTTTTCAGATGG

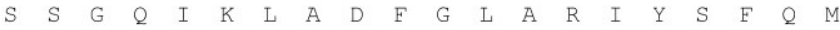

CTCTTACCTCAGTGGTTGTCACTCTGTGGTACAGAGCTCCTGAAGTTTTGCTTCAATCCA

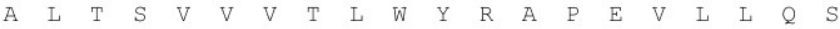

GTTATGCAACACCAGTTGATCTCTGGAGTGTTGGCTGCATCTTTGAACAAATGTTAACCC

$\begin{array}{llllllllllllllllllll}S & Y & A & T & P & V & D & L & W & S & V & G & C & I & E & E & \& & M & L & T\end{array}$

СCСТAAAC

P L $\quad \mathrm{N} O$

B)

C)
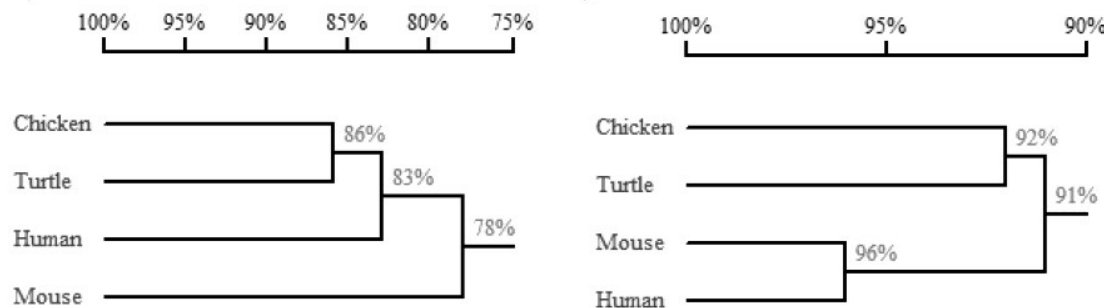

Figure 2.18. Nucleotide and deduced amino acid sequence for T. s. elegans partial $c d k 6$ sequence. Nucleotides and amino acids are numbered on the left. (A) Nucleotides and amino acids are numbered on the left. The nucleotide sequence was 548 nucleotides in length, while the amino acid sequence was 182 amino acids in length. Homology of (B) nucleotide and (C) protein sequences of the turtle to that of chicken (G. gallus), Mouse (M. musculus) and human (H. sapiens). 
A)

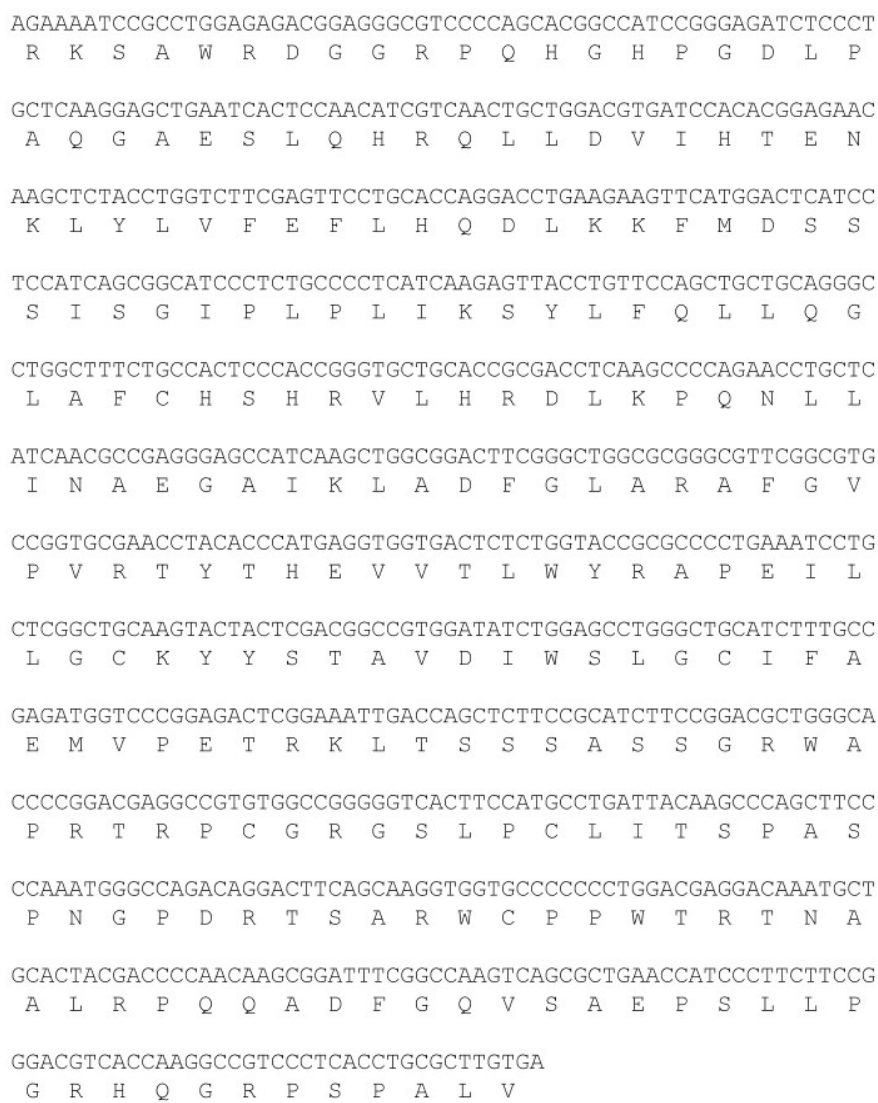

B)
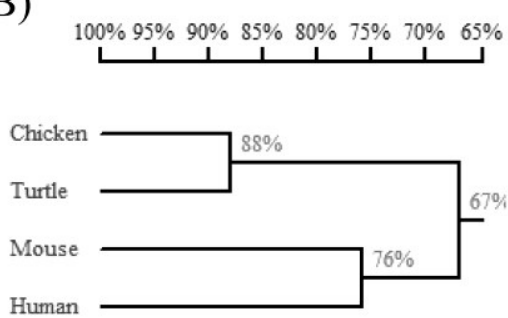

C)

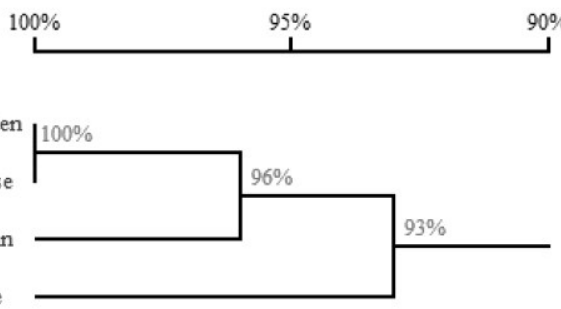

Figure 2.19. Nucleotide and deduced amino acid sequence for T. s. elegans partial $c d k 2$ sequence. Nucleotides and amino acids are numbered on the left. (A) Nucleotides and amino acids are numbered on the left. The nucleotide sequence was 757 nucleotides in length, while the amino acid sequence was 252 amino acids in length. Homology of (B) nucleotide and (C) protein sequences of the turtle to that of chicken (G. gallus), Mouse (M. musculus) and human (H. sapiens). 
A)

AGGTACCTATGGTGTTGTATATAAGGGTCGACACAAATCCACAGGCCAGGTGGTTGCCAT

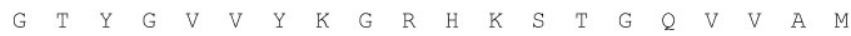

GAAGAAAATTCGACTAGAAAGTGAGGAAGAAGGTGTTCCCAGTACAGCAATCAGGGAAAT $\begin{array}{llllllllllllllllllll}K & K & I & R & L & E & S & E & E & E & G & V & P & S & T & A & I & R & E & I\end{array}$

TTCTTTACTAAAAGAGCTACACCATCCCAATATAGTCTGTCTTCAGGATGTGCTTATGCA

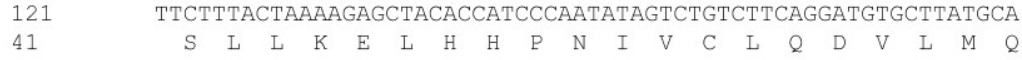

181 AgATTCAAGACTGTACCTCATCTTTGAATTCCTTTCTATGGATCTCAAAAAATATTTGGA $\begin{array}{lllllllllllllllllllll}61 & D & S & R & L & Y & L & I & F & E & F & L & S & M & D & L & K & K & Y & L & D\end{array}$ 241 CTCTATTCCATCTGGCCAAGAGTTACCTGTATCAAATCTTGCAAGGTATTGTATTCTGTC

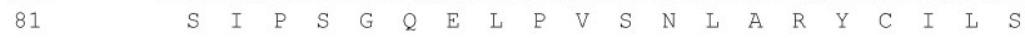
301 ATTCAAGAAGGGTTCTGCACAGAGATTAAAGCCTCAAAACTTGTTAATAGATGATAAAG

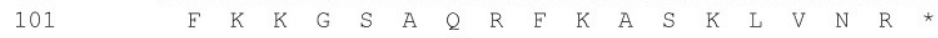

361 GAGTAATTAAATTAGCAGATTTTGGACTGGCCCGAGCCTTTGGGATCCCTGTCCGGGTAT

421 ATACACATGAgTAgTGACACTGTGGTATAGATCTCCAGAGGTGTTGCTAGGATCTGCTCG

481 TTACTCAACTCCTGTAGATATCTGGAGCATTGGTACCATATTTGCTGAAATTGCAACTAA

541 GAAACCACTCTTTCATGGAGACTCTGAAATTGATCAACTCTTCAGAATCTTCAgAGCTTT

601 AGGGACACCCAACAATGAGGTGTGGCCCGAAGTGGAATCCTTGCAAGACTATAAGAACAC

661 ATTCCCCAAGTGGAAACCTGGCAGTCTGGCGTCTCATGTCAAAAACTTAGATGAAGATGG

721 ACTAAAATGTTAATTTATGATCCTGCAAAAAGAATCTCTGGCAAAATGGCCTTGAACCAT

781 CCATACTTTGATGACTTGGACAAATCCAATCTTCCAGCCAATCAGATTAAGAAATTCTAA

B)

C)
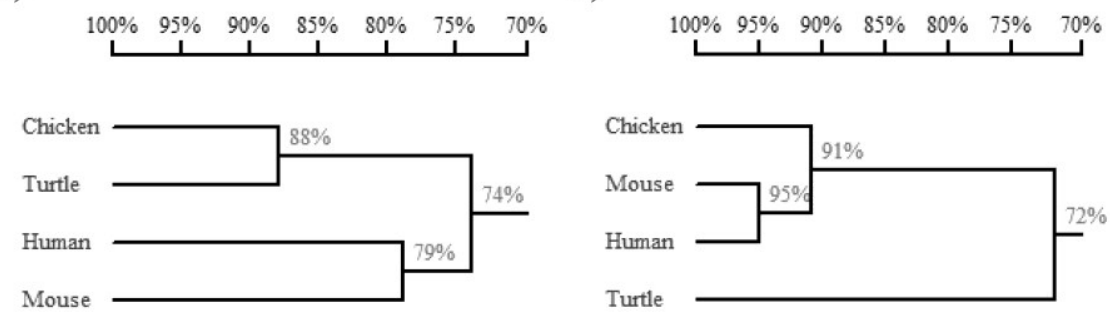

Figure 2.20. Nucleotide and deduced amino acid sequence for $T$. s. elegans partial $c d c 2$ sequence. Nucleotides and amino acids are numbered on the left. (A) Nucleotides and amino acids are numbered on the left. The nucleotide sequence was 840 nucleotides in length, while the amino acid sequence was 117 amino acids in length. Homology of (B) nucleotide and $(\mathrm{C})$ protein sequences of the turtle to that of chicken (G. gallus), Mouse (M. musculus) and human (H. sapiens). 


\section{CHAPTER 3}

\section{REGULATION OF THE $G_{1}$ PHASE OF THE CELL CYCLE}




\subsection{Introduction}

What are the mechanisms for cell cycle arrest induced by oxygen restriction? Researchers have generalized numerous hypoxia-sensitive pathways and checkpoints in cell cycle regulation in several organisms, highlighting the multiple interconnected pathways at play and further emphasizing the importance of an appropriate model system. Recent studies that have examined the state of the cell cycle under hypoxic stress have largely been limited to the early developmental stages of brine shrimp (Artemia franciscana) embryos, zebrafish (Danio rerio) embryos, nematodes (Caenorhabditis elegans) and fruit flies (Drosophila melanogaster) (Douglas and Haddad, 2003). As such, research focusing on the developing nematode has found that larval stages can enter a reversible suspended animation at all stages of the cell cycle when presented with an anoxic stress (Padilla et al., 2002; Douglas and Haddad, 2003). Similar to the research carried out with nematodes, studies utilizing GFP-kinesin in the early embryonic stages of fruit fly development have shown that hypoxia (less than $2 \%$ oxygen) induces a prolongation of all cell cycle stages. Furthermore, when exposed to anoxia these embryos enter reversible arrest at one of two phases: mitosis (aligned, non-segregated chromatids) and a $\mathrm{G}_{1}$-like phase (early embryos do not possess a true $\mathrm{G}_{1}$ phase) (Douglas and Haddad, 2003). Although complete analysis of the cell cycle has not yet been preformed, it is known that during the embryonic stages, brine shrimp can enter a quiescent state $\left(\mathrm{G}_{0}\right)$ for several years; a state facilitating the long-term arrest of the cell cycle and allowing for development to resume when environmental conditions become favorable (Clegg, 1997; Hand, 1998; Douglas and Haddad, 2003). Additionally, zebrafish in their early embryonic stages have been found to enter a state of reversible arrest when presented 
with an anoxic environment (Padilla and Roth, 2001). Flow cytometric studies have indicated that these embryos enter an arrest in the $G_{1}$ and $G_{2}$ phases of the cell cycle. It has been postulated that these phases of the cell cycle may have a higher oxygen demand than other phases (Padilla and Roth, 2001). Although there have been several studies characterizing the anoxic response in developing organisms, cell cycle arrest has not yet been characterized for developmentally mature vertebrates tolerant of severe hypoxia and/or full anoxia.

Hypoxia research has received much research attention since it is physiologically relevant, occurring during normal embryogenesis, ischemic injuries, and tumor progression (Hammond and Giaccia, 2004). Given the clinically important implications of hypoxia, the proper selection of a model system becomes critical. In order to address issues in hypoxic cell cycle regulation there needs to be more focused research carried out in non-model organisms that better facilitate the oxygen stress response pathway. Proliferation and/or replacement of cells are clearly energy-expensive biosynthetic processes, so it would make sense that these activities are suppressed to a minimum under conditions of severe oxygen restriction. Taking into account the findings presented in Chapter 2, along with reports by Mazia (1962) who suggested that withdrawal of energy yielding substances will induce $\mathrm{G}_{1}$ arrest if applied before S-phase, an arrest of the cell cycle during anoxia exposure in turtles seems highly probable. The demanding energetics of mitosis provides an intriguing suggestion that the cell cycle may arrest in proliferating tissues in order to facilitate metabolic rate depression and play an important role in cellular responses to environmental stresses. 
Indeed, studies in other systems of facultative metabolic arrest, such as mammalian hibernation, do show cell cycle arrest when organisms enter hypometabolic states. For example, studies with hibernating ground squirrels (Citellus undulatus) indicated that highly regenerative tissues such as intestinal epithelium arrest in the $2 \mathrm{~N}$ DNA range $\left(\mathrm{G}_{1} / \mathrm{G}_{0}\right)$ (90\% of the total cell population) during hibernation as compared to active states ( $79 \%$ of the total cell population), whereas both $\mathrm{S}$ and $\mathrm{G}_{2} / \mathrm{M}$ phases are decreased during hibernation (Kruman et al. 1986; Kolaeva et al., 1980). These results suggest that DNA synthesis is markedly reduced during the hibernation period. Similarly, Douglas et al. (2001) have shown that in the hypoxia tolerant fruit fly, D. melanogaster, embryos can arrest development and remain suspended in $\mathrm{G}_{1} / \mathrm{G}_{0}$ phase when they are deprived of oxygen. Their study went further to describe a hypoxia-specific checkpoint in D. melanogaster where mitogenic factors contribute to $\mathrm{Cdk}$ inactivation and lead to $\mathrm{G}_{1}$ arrest. In other species with varying degrees of hypoxia tolerance, sites of cell cycle arrest appear to differ. Research presented in Chapter $\mathbf{2}$ suggested that cells of the anoxic turtle may arrest the cell cycle, but did not explore specific states (such as a pause of the cell cycle, $\mathrm{G}_{1}$ arrest or reversible escape into $\mathrm{G}_{0}$ ) or suggest any possible mechanisms of arrest. It is the goal of this Chapter to further elucidate the possible arrest mechanisms utilized by the turtle and further define cell cycle regulation of Cdk proteins during periods of anoxic stress.

The previous chapter presented a summary of the critical Cyclins and Cdks involved in driving the cell cycle, as well as the activation of a signaling pathway that controls cell cycle initiation. Overall, there was a clear decrease in the relative expression of both total and nuclear Cyclin and Cdk proteins in response to anoxia. This may be 
controlled, in part, by an initial decrease in cell cycle signaling via the PI3K/Akt pathway and Cyclin D1 regulation. Although it is clear from these results that the cell cycle is suppressed, it is unclear whether these data are a result of phase-specific arrest mechanisms. These phase-specific mechanisms should contribute greatly to determining overall cell cycle regulation. Mechanisms of $\mathrm{G}_{1}$ and $\mathrm{G}_{2}$ specific cell cycle arrest are investigated in this chapter.

\subsubsection{Regulation of $G_{1}$ phase of the cell cycle}

In addition to cyclin-dependent regulation of Cdk activity, Cyclin/Cdk complexes are controlled via various inhibitory proteins, the CKIs. These inhibitors have been classified into two families, INK4 and Cip/Kip, based on their mechanisms of inhibition (Sherr and Roberts, 1995a; Sherr and Roberts, 1995b). The INK4 family of Cdk inhibitors (primarily p16, but also p15, p18 and p19) has been found to regulate monomeric $\mathrm{Cdk} 4$ and $\mathrm{Cdk} 6$. P16 binding to a Cdk overlaps the $\mathrm{Cdk}$ region responsible for Cyclin binding, thus blocking the formation of the Cyclin/Cdk complex and inhibiting Cdk function (Obaya and Sedivy, 2002; Coleman et al., 1997). The Cip/Kip family of inhibitors (p21 and p27) inhibits both monomeric Cdks and Cyclin/Cdk complexes (Toyoshima and Hunter, 1994; Harper et al., 1993). Binding of the Cip/Kip family to Cdks can completely shut down the active Cyclin/Cdk complex by antagonizing the interactions of ATP substrate, into the Cdk catalytic cleft (Pavletich, 1999) (Figure 3.1).

Both the INK4 and Cip/Kip CKIs are expressed during gap phases of the cell cycle (Guardavaccaro and Pagano, 2006). A decrease in p27 and p21 allows for increased formation of active Cyclin/Cdk complexes, leading to the subsequent phosphorylation of 
$\mathrm{Rb}$, activation of E2F, and re-entry into the cell cycle (Figure 2.2). The combinatory effects of CKIs allows for self-maintenance and entry into quiescence $\left(\mathrm{G}_{0}\right)$. It has been suggested that it is primarily the decreased expression of CKIs that allows re-entry into the cell cycle (Kwon et al., 2002). Similar to Cyclins, the expression profile of CKIs are specific to the arrest mechanism. The increased expression of p21 is typically associated with $\mathrm{G}_{1}$ and $\mathrm{G}_{2}$ arrested cells, while p27 and p16 are specific to $\mathrm{G}_{1}$ and $\mathrm{G}_{0}$ arrested cells. An expression profile of CKIs could provide an indication of the specific type of cell cycle arrest utilized by the anoxic turtle.

\subsubsection{Regulation of the cell cycle through DNA damage checkpoints}

As mentioned in Chapter 2, Cdks may be phosphorylated on both Thr14 and Tyr15, inhibiting kinase activity and aiding arrest of cellular proliferation. Also, phosphorylation at these residues was found to be anoxia responsive since relative phosphorylation increased in all tissue during anoxia. Counteracting this inhibitory phosphorylation is dephosphorylation mediated by the Cdc25a and Cdc25c phosphatases. These Cdk activators are able to dephosphorylate both Thr14 and Tyr15 residues, and are commonly over-expressed in human cancers.

DNA checkpoints in the cell cycle preserve accurate replication and report DNA damage. These checkpoint pathways ensure error-free DNA replication and chromosome segregation, thereby tightly regulating cell cycle transitions and ensuring the maintenance of genomic integrity. The signaling of these checkpoints are transduced by apical signal kinases such as PI3K-like family members, ataxia telangiectasia mutated (ATM) and ataxia telangiectasia and rad3 related (ATR) kinases. These kinases regulate downstream 
distal serine/threonine signal transducing kinases, checkpoints 1 and 2 (Chk1 and Chk2, respectively). Finally, these distal kinases regulate the diverse group of effector proteins encompassing cell cycle regulators such as $\mathrm{Cdc} 25 \mathrm{a}$ and $\mathrm{Cdc} 25 \mathrm{c}$ phosphatases, p53 and Cyclin/Cdk complexes (Figure 3.2).

The kinase activity of ATM is activated in response to double-stranded DNA breaks, and ATM targets several effectors of checkpoint control, including Chk2 and p53 (Powers et al., 2004). Cells lacking ATM are hypersensitive to ionizing radiation (IR) but not to ultraviolet (UV), whereas cells overexpressing a kinase-inactive form of ATR are sensitive to UV and IR (Piret et al., 1999). This suggests that ATR plays a more prominent role than ATM during the cellular response to stress or to certain types of DNA damage. To prevent inappropriate entry into both $\mathrm{S}$ and $\mathrm{M}$ phases, cells progressing though the $\mathrm{G}_{1}$ and $\mathrm{G}_{2}$ phases, respectively, activate the checkpoint transducing kinases ATM/ATR and Chk1/Chk2. Both ATR and ATM are able to activate Chk2 through initial phosphorylation of Ser19, followed by an auto-phosphorylation event at Thr387 and complete activation. The primary targets of activated Chk2 include two critical effectors mediating the $\mathrm{G}_{1}$ checkpoint, Cdc25a and p53 (Figure 3.2) (Choudhury et al., 2007). Targeting of Cdc25a, the protein primarily responsible for Cdk2 activation, by Chk2 leads to enhanced ubiquitination and proteasome-mediated degradation (Falck et al., 2001). In contrast to Cdc25a, p53 is stabilized through its interactions with Chk2, leading to enhanced transcriptional activity (Choudhury et al., 2007). The key cell cycle regulator that is upregulated by p53-mediated transcription is the Cdk inhibitor, p21 (see previous). Accumulation of $\mathrm{p} 21$ is capable of inducing cell cycle arrest by blocking Cdk2 activity. 
The $\mathrm{G}_{2}$ checkpoint prevents cells from entering mitosis when they experience DNA damage and is primarily regulated through Chk1 activity (Shibat et al., 2009). Similar to Chk2, Chk1 can be activated through phosphorylation by either ATM or ATR and can act to inhibit Cdc25a and Cdc25c function. Activation of Chk1 involves phosphorylation at Ser317 and Ser345. The key regulatory complex of the $\mathrm{G}_{2}$ checkpoint is the activity of the Cyclin B1/Cdc2 complex. Activation of Cyclin B1/Cdc2 is prevented primarily through Chk1-mediated inhibition of $\mathrm{Cdc} 25 \mathrm{c}$, responsible for Cdc2 activation at the $\mathrm{G}_{2} / \mathrm{M}$ boundary (Shibat et al., 2009). Ultimately, the ATM/ATR checkpoint pathway mediates its effects by inhibiting Cdks, the primary motors of the cell cycle (Figure 3.2).

Although hypoxia does not itself induce DNA damage or a typical DNA damage response, several studies have indicated that the phosphorylation and activation of Chk2 occurs in an ATM-dependent manner during hypoxia (Bencokova et al., 2009; Hammond and Giaccia, 2004). Although the mechanisms for this arrest have not been clearly defined, hypoxia induced a rapid $\mathrm{G}_{1}$ arrest similar to that induced through DNA damage (Bencokova et al., 2009). In addition, hypoxia-activated Chk2 induced cell cycle arrest through similar mechanisms as seen for DNA damage; this included the activation and stabilization of $\mathrm{p} 53$ and targeting Cdc25a for degradation. Activation of Chk2 may prove to be a crucial mechanism initiating hypoxic cell cycle arrest.

The present chapter focuses on the regulation of Cdk proteins characterizing the expression of both Cdk activators (Cdc25a and Cdc25c) and inhibitors (p16, p27 and p21) in response to anoxia in turtle organs. Additionally, the chapter characterizes the 
potential activation of a stress-responsive cell cycle arrest pathway (ATM/ATR), known to regulate $\mathrm{Cdc} 25 \mathrm{a}$ and $\mathrm{Cdc} 25 \mathrm{c}$ activity. 


\subsection{Materials and Methods}

\subsubsection{Animal Care and Treatment}

All animal experiments were conducted as described in Chapter 2.

\subsubsection{Total protein and nuclear isolation}

All protein isolations were conducted as described in Chapter $\mathbf{2}$.

\subsubsection{Immunoblotting}

Preparation of tissue extracts and immunoblotting were performed as described in Chapter 2 with minor modifications. For immunoblotting, aliquots containing $20 \mu \mathrm{g}$ protein from control and anoxic ( 5 and $20 \mathrm{~h}$ ) conditions were loaded into lanes of polyacrylamide gels (8-15\% gels depending on the MW of the protein being analyzed). Gels were run in a Mini Protean III apparatus (BioRad) at $180 \mathrm{~V}$ for either $45 \mathrm{~min}$ at RT or 60 min at $4{ }^{\circ} \mathrm{C}$ (for ATM and ATR immunoblots). The resolved proteins were transferred onto PVDF membranes $(0.2 \mu \mathrm{m}$ pore size for $\mathrm{p} 16, \mathrm{p} 21$ and $\mathrm{p} 27$ immunoblots; $0.45 \mu \mathrm{m}$ for all other proteins) at $70 \mathrm{~V}$ for $90 \mathrm{~min}$ at $4{ }^{\circ} \mathrm{C}$. The transfer was carried out at $160 \mathrm{~mA}$ constant amperage for $1.5 \mathrm{~h}$. The membranes were blocked using $1 \mathrm{mg} / \mathrm{ml} \mathrm{PVA}$ (70-100 kDa) in TBST for 30-45 s. After blocking, the membranes were probed with an antigen-specific antibody (see Appendix C). Experimental conditions that are specific to each antibody are listed in Appendix D. Immunoblots were developed using enhanced chemiluminescence reagents. 


\subsubsection{Total RNA isolation}

RNA extraction was performed as described in Chapter 2.

\subsection{5 cDNA synthesis}

cDNA synthesis were performed as described in Chapter 2.

\subsubsection{Polymerase chain reaction}

PCR amplification was performed as described in Chapter 2. Polymerase Chain Reaction (PCR) was used to amplify the sequences under study from the cDNA samples. Each PCR reaction consisted of $13.25 \mu \mathrm{L}$ of sterile water, $5 \mu \mathrm{L}$ of diluted cDNA, $1.25 \mu \mathrm{L}$ of $1.5 \mu \mathrm{M}$ primer mixture (see Appendix F), $2.5 \mu \mathrm{L}$ of $10 \times$ PCR buffer (Invitrogen; Cat\# N8080006), $1.5 \mu \mathrm{L}$ of $50 \mathrm{mM} \mathrm{MgCl} 2,0.5 \mu \mathrm{L}$ of $25 \mathrm{mM}$ dNTPs and $1 \mu \mathrm{L}$ of Taq polymerase, for a total volume of $25 \mu \mathrm{L}$. All PCR amplification cycles were as follows; an initial denaturation at $94{ }^{\circ} \mathrm{C}$ for $7 \mathrm{~min}$, followed by an experimentally determined number of cycles of $94^{\circ} \mathrm{C}$ for 1 min (see Appendix G), primer annealing $50-60{ }^{\circ} \mathrm{C}$ for 1 min, and elongation at $72{ }^{\circ} \mathrm{C}$ for $1 \mathrm{~min}$. The final elongation was at $72{ }^{\circ} \mathrm{C}$ for $10 \mathrm{~min}$.

\subsubsection{Sequencing}

All PCR products were sequenced by BioBasics (Markham, ON) as described in

\section{Chapter 2.}

\subsubsection{Statistics}

Data analysis was performed as described in Chapter 2. 


\subsection{Results}

\subsubsection{Regulation of ATM/ATR protein signaling}

Changes in the relative expression of total ATM and ATR proteins in response to anoxia were assessed using immunoblotting. All antibodies cross-reacted with a protein band of either $\sim 350 \mathrm{kDa}$ or $\sim 250 \mathrm{kDa}$, the expected sizes of ATM and ATR, respectively (Figure 3.3). Relative protein expression of ATM significantly increased in response to 5 and $20 \mathrm{~h}$ anoxia in liver by $1.66 \pm 0.10$ and $1.93 \pm 0.06$-fold over control values, respectively (Figure 3.4) $(P<0.05)$. No significant changes in the relative protein expression of ATM were detected in kidney. However, in white skeletal muscle, ATM levels decreased to $78 \pm 3 \%$ of control values in response to $5 \mathrm{~h}$ anoxia $(P<0.05)$ but returned to control values after $20 \mathrm{~h}$ anoxia. No significant changes in total ATR expression were detected in any of the three tissues in response to $5 \mathrm{~h}$ anoxia or after $20 \mathrm{~h}$ in kidney and muscle (Figure 3.4). However, ATR protein levels decreased in liver to $69 \pm 5 \%$ of control values after $20 \mathrm{~h}$ anoxia.

\subsubsection{Total protein and phosphorylation regulation of Chk1 and Chk2}

Expression levels of checkpoint (Chk) proteins (types 1 and 2) revealed differential regulation in both protein expression and phosphorylation in liver, kidney and white skeletal muscle from turtles in response to 5 and $20 \mathrm{~h}$ anoxia exposure (Figure 3.5). The checkpoint protein, Chk1 (immunoreactive band of $\sim 56 \mathrm{kDa}$; Figure 3.3), was found to be differentially regulated in all tissues in both protein expression and phosphorylation level. In liver tissue, total Chk1 protein content significantly decreased to $65 \pm 8$ and $66 \pm 4 \%$ of control values in response to 5 and $20 \mathrm{~h}$ anoxia, respectively $(P<0.05)$. In 
kidney, the relative expression of Chk1 increased by $1.37 \pm 0.04$-fold over control values after $5 \mathrm{~h}$ anoxia $(P<0.05)$ but returned to control levels after $20 \mathrm{~h}$ anoxia. In white skeletal muscle, relative expression of Chk1 protein significantly increased by $1.43 \pm 0.08$ fold from control values after $5 \mathrm{~h}$ anoxia $(\mathrm{P}<0.05)$ but, as in kidney, returned back to control values after $20 \mathrm{~h}$ anoxia exposure. In response to either 5 or $20 \mathrm{~h}$ anoxia, no significant changes in the relative expression of p-Chk1 (Ser317) were detected in either kidney or liver tissue. However, in white skeletal muscle, the relative expression of $\mathrm{p}$ Chk1 (Ser317) increased by $1.23 \pm 0.05$-fold in response to $5 \mathrm{~h}$ anoxia; expression levels significantly decreased back to control values after $20 \mathrm{~h}$ anoxia $(P<0.05)$. No significant change in the phosphorylation state of p-Chk1 (Ser345) was detected in liver in response to $5 \mathrm{~h}$ anoxia and values significantly decreased to $82 \pm 3 \%$ of control levels after $20 \mathrm{~h}$ of anoxia $(P<0.05)$. A significant increase in $\mathrm{p}-\mathrm{Chk} 1$ (Ser345) expression after $20 \mathrm{~h}$ anoxia of $1.48 \pm 0.04$-fold over control values was seen in kidney tissue $(P<0.05)$. In white skeletal muscle a significant increase in p-Chk1 (Ser345) was detected in response to 5 and $20 \mathrm{~h}$ anoxia, levels were $1.55 \pm 0.09$ and $1.46 \pm 0.15$-fold higher than control values, respectively $(P<0.05)$.

The checkpoint protein, Chk2 (immunoreactive band of $\sim 62 \mathrm{kDa}$; Figure 3.3), was regulated in a similar fashion in all three tissues studied (Figure 3.5). In liver, the expression of total Chk2 protein significantly increased by $1.29 \pm 0.07$ and $1.59 \pm 0.09$-fold from control values in response to 5 and $20 \mathrm{~h}$ anoxia, respectively $(P<0.05)$. Similarly total Chk2 protein reached $1.85 \pm 0.16$-fold over control values after $20 \mathrm{~h}$ anoxia $(P<$ 0.05). In white skeletal muscle the expression of total Chk2 protein significantly increased by $1.33 \pm 0.06$ and $1.96 \pm 0.16$-fold from control values in response after 5 and 20 
$\mathrm{h}$ anoxia, respectively $(P<0.05)$. In response to either 5 or $20 \mathrm{~h}$ anoxia, no significant changes in the relative amount of p-Chk2 (Ser19) were detected in either liver or white skeletal muscle. However, in kidney the relative expression of p-Chk2 (Ser19) decreased to $59 \pm 8$ and $65 \pm 2 \%$ of control values in response to 5 or $20 \mathrm{~h}$ anoxia, respectively $(P<$ 0.05). Similar expression patterns for $\mathrm{p}-\mathrm{Chk} 2$ (Ser387) were detected in response to anoxia for liver and kidney. In liver, the relative expression of p-Chk2 (Ser387) significantly increased by $1.26 \pm 0.1$ and $1.62 \pm 0.1$-fold from control values in response to 5 and $20 \mathrm{~h}$ anoxia, respectively $(P<0.05)$ whereas in kidney p-Chk2 (Ser387) content rose by $1.24 \pm 0.02$ and $1.62 \pm 0.11$-fold, respectively $(P<0.05)$. However, in white skeletal muscle the relative expression of p-Chk2 (Ser387) decreased to $71 \pm 6$ and $65 \pm 7 \%$ of control values in response to 5 and $20 \mathrm{~h}$ anoxia, respectively $(P<0.05)$.

\subsubsection{Total cellular protein expression of Cdk regulators, $\mathrm{Cdc25a}$ and $\mathrm{Cdc25c}$}

Changes in the relative expression of total $\mathrm{Cdc} 25 \mathrm{a}$ and $\mathrm{Cdc} 25 \mathrm{c}$ protein in response to anoxia were assessed using immunoblotting (Figure 3.6). All antibodies cross-reacted with a protein band of either $\sim 65 \mathrm{kDa}$ or $\sim 60 \mathrm{kDa}$, the expected size of Cdc25a and Cdc25c, respectively (Figure 3.3). The expression pattern of Cdc25a protein was similar in all three tissues studied. In liver, the expression of total Cdc25a protein significantly decreased to $64 \pm 7$ and $53 \pm 1 \%$ of control values in response to 5 and $20 \mathrm{~h}$ anoxia, respectively $(P<0.05)$. Kidney showed the same pattern with total Cdc25a protein significantly decreased to $47 \pm 2$ and $41 \pm 2 \%$ of control values after 5 and $20 \mathrm{~h}$ anoxia, respectively $(P<0.05)$ whereas total $\mathrm{Cdc} 25$ a protein in white skeletal muscle significantly decreased to $68 \pm 2$ and $75 \pm 8 \%$, respectively $(P<0.05)$. 
The relative expression profile of $\mathrm{Cdc} 25 \mathrm{c}$ was similar to that of $\mathrm{Cdc} 25 \mathrm{c}$ and also similar between the three tissues (Figure 3.6). In liver, expression of total Cdc $25 \mathrm{c}$ protein significantly decreased to $75 \pm 4$ and $55 \pm 8 \%$ of control values in response to 5 and $20 \mathrm{~h}$ anoxia, respectively $(P<0.05)$. Kidney Cdc25c protein levels significantly decreased to $62 \pm 3$ and $62 \pm 1 \%$ of control values after 5 and $20 \mathrm{~h}$ anoxia, respectively $(P<0.05)$ whereas white skeletal muscle showed significantly decreases to $76 \pm 8$ and $54 \pm 5 \%$, respectively $(P<0.05)$.

\subsubsection{Total cellular protein expression and phosphorylation status of Cdk inhibitors, p27, p16 and p21}

Expression levels of Cdk inhibitor proteins revealed differential trends in protein expression in liver, kidney and white skeletal muscle from turtles in response to 5 and 20 h anoxia exposure (Figure 3.7). The Cip/Kip family Cdk inhibitor protein, p27 (immunoreactive band of $\sim 27 \mathrm{kDa}$; Figure 3.3), was differentially regulated in all tissues. Expression of total p27 protein significantly increased by $3.03 \pm 0.19$-fold from control values after $20 \mathrm{~h}$ anoxia in liver tissue $(P<0.05)$ but no change was seen after $5 \mathrm{~h}$ anoxia. By contrast, protein expression of p 27 decreased strongly to $22 \pm 9$ and $29 \pm 7 \%$ of control values in kidney after 5 and $20 \mathrm{~h}$ anoxia, respectively $(P<0.05)$. No significant changes in $\mathrm{p} 27$ protein expression were detected in white skeletal muscle. The relative phosphorylation of p27 on residue Thr187 was assessed using antibodies specific to the epitope of the phosphorylation site. In liver tissue, the relative expression of p-p27 (Thr187) was reduced after both 5 and $20 \mathrm{~h}$ anoxia to $54 \pm 5$ and $67 \pm 5 \%$ of control values, respectively $(P<0.05)$. Relative expression of p-p27 (Thr187) in kidney was also reduced after both 5 and $20 \mathrm{~h}$ anoxia, falling to $27 \pm 5$ and $23 \pm 4 \%$ of control values, 
respectively $(P<0.05)$. No significant changes in phosphorylated $\mathrm{p} 27$ content were detected in white skeletal muscle.

The INK4 family Cdk inhibitor protein, p16 (immunoreactive band of $\sim 16 \mathrm{kDa}$; Figure 3.3), was also differentially regulated in all tissues (Figure 3.7). Expression of total p16 protein significantly increased in both 5 and $20 \mathrm{~h}$ anoxic liver by $1.83 \pm 0.16$ and $1.34 \pm 0.4$-fold over control values, respectively $(P<0.05)$. In kidney, the protein expression of $\mathrm{p} 16$ increased by $1.50 \pm 0.08$-fold compared with controls after $20 \mathrm{~h}$ anoxia $(P<0.05)$. No significant changes in $\mathrm{p} 16$ protein expression were detected in white skeletal muscle. The relative phosphorylation of p16 on residue Thr152 was assessed using antibodies specific to the epitope of the phosphorylation site. Relative expression of p-p16 (Thr152) rose dramatically in 5 and $20 \mathrm{~h}$ anoxic liver by $7.72 \pm 0.46$ and $4.38 \pm 0.94$ fold over control values, respectively $(P<0.05)$. In kidney tissue, p-p16 (Thr152) levels increased by $1.33 \pm 0.13$-fold over control values after $20 \mathrm{~h}$ anoxia $(P<0.05)$ but no significant changes were detected in white skeletal muscle.

The Cip/Kip family Cdk inhibitor protein, p21 (immunoreactive band of $\sim 21 \mathrm{kDa}$; Figure 3.3), did not change significantly relative to control in response anoxia in any studied tissue (Figure 3.7). However, relative phosphorylation of p21 on residue Thr145 did respond to anoxia and displayed similar expression patterns in anoxic liver and kidney. Relative expression of p-p21 (Thr145) significantly increased in 5 and $20 \mathrm{~h}$ anoxic liver by $1.55 \pm 0.17$ and $2.98 \pm 0.11$-fold from control values, respectively $(P<0.05)$ whereas in kidney, comparable values were $1.72 \pm 0.07$ and $2.90 \pm 0.02$-fold, respectively $(P<0.05)$. However, the relative expression of p-p21 (Thr145) decreased after $20 \mathrm{~h}$ 
anoxia to $53 \pm 4 \%$ of control values in white skeletal muscle $(P<0.05)$; no changes occurred after $5 \mathrm{~h}$ anoxia.

\subsubsection{Transcript expression of Cdk inhibitor, p27}

Using RT-PCR and primers derived from the consensus sequence of $p 27$ from several other vertebrates, the relative expression of $p 27$ transcripts was assessed in response to anoxia in the turtle (Figure 3.8). In liver tissue, relative transcript expression of $p 27$ decreased significantly after $5 \mathrm{~h}$ anoxia to $46 \pm 3 \%$ of control values $(\mathrm{P}<0.05)$ but after $20 \mathrm{~h}$ anoxia, p27 transcript levels had increased significantly as compared with both control and $5 \mathrm{~h}$ anoxia levels, rising to $1.92 \pm 0.21$-fold over control values $(P<0.05)$. Relative transcript expression of $p 27$ decreased significantly after $20 \mathrm{~h}$ anoxia to $64 \pm 4 \%$ of control values in kidney tissue $(P<0.05)$ but white skeletal muscle showed no significant change in $p 27$ transcript expression in response to anoxia. The amplified transcript and associated translated protein sequence for the $p 27$ amplicon is presented in Figure 3.9A. The amplified turtle sequence represented 37\% mRNA coverage and displayed 69\% homology to the corresponding human sequence (Figure 3.9B). The translated turtle protein sequence represented 59\% coverage and displayed $63 \%$ homology to the corresponding human protein sequence (Figure 3.9C). 


\subsection{Discussion}

The cell cycle is comprised four distinct phases, $G_{1}, S, G_{2}$, and $M$ phase that control and regulate the progression of cell division (see Chapter 2, Figure 2.1). The progression through each phase of the cell cycle is tightly regulated by two classes of proteins: Cyclins and Cyclin dependent kinases (Cdks) (Schafer, 1998). The binding of the regulatory Cyclin proteins to Cdks is essential in the formation of the kinase complex, which upon dephosphorylation by $\mathrm{Cdc} 25$ phosphatases, fully activates the kinase activity of the Cyclin/Cdk complex (Bloom and Cross, 2007; Ohtani and Nevins, 1994). The activated Cdk functions to phosphorylate a number of different substrates, catalyzing the progression of cell division (Schafer, 1998). The complexity of eukaryotic cell cycle regulation derives from the multiple Cyclins that interact with multiple Cdks, with each distinct combination acting to regulate a specific phase of the cell cycle (Bloom and Cross, 2007).

Although it is clear from the results presented in Chapter 2 that the cell cycle is regulated during anoxia in turtle organs, the mechanisms of this regulation still remained unclear. The goal of this chapter was to determine possible modes of Cdk regulation, characterizing the expression of Cdk activators (Cdc25a and $\mathrm{Cdc} 25 \mathrm{c})$ and inhibitors $(\mathrm{p} 16$, p27 and p21) as well as the activation of DNA damage response pathways (ATM and ATR) in response to anoxia.

\subsubsection{Cell cycle regulation by ATM/ATR DNA damage response pathways}

Although the oscillating patterns of cyclin proteins are often good indicators of cell cycle progression, complex regulatory systems also exist that control the activity of 
Cyclin/Cdk complexes. Most notably, the Cyclin/Cdk complexes are tightly regulated by check point kinases (Chk), Cdc25 phosphatases and several CKIs. Both Chk1 and Chk2, are regulated and activated via phosphorylation by ataxia telangiectasia and rad3 related (ATR) and ataxia telangiectasia mutated (ATM) proteins, two stress response protein kinases that are known to be triggered by UV or IR induced DNA damage or hypoxia (Abraham, 2001). As seen in Figure 3.4, only the relative expression of ATM (and not ATR) increased in response to 5 and $20 \mathrm{~h}$ anoxia in liver tissue. The relative expression of both ATM and ATR were not found to significantly decrease in response to anoxia in kidney tissue; no changes were found after $20 \mathrm{~h}$ anoxia in white skeletal muscle. The protein expression levels of ATM or ATR are not indicative of pathway activation themselves but may suggest an ability to elicit a stronger stress response to activate downstream checkpoint proteins in anoxic liver tissue via ATM. To determine the state of ATM or ATR activation, the relative phosphorylation of downstream targets of ATM and ATR were assessed: both Chk1 (residues Ser317 and Ser345) and Chk2 (residue Ser19). As seen in Figure 3.5, phosphorylation-mediated activation of Chk1 was only found in kidney and white skeletal muscle, as determined by relative increases in $\mathrm{p}-\mathrm{Chk}$ (Ser345); there was no indication of Chk1 activation in liver. By contrast, in response to anoxia, Chk2 was activated in liver and kidney tissues, as determined by relative changes in Chk2 auto-phosphorylation at residue Thr387. The increase in Chk1 phosphorylation during anoxia in both kidney and white skeletal muscle is interesting as active Chk1 functions as a regulator of both $\mathrm{G}_{1}$ and $\mathrm{G}_{2} / \mathrm{M}$ checkpoints by inactivating both Cdc25a $\left(\mathrm{G}_{1}\right.$ and $\mathrm{G}_{2}$ checkpoints) and $\mathrm{Cdc} 25 \mathrm{c}\left(\mathrm{G}_{2} / \mathrm{M}\right.$ checkpoints) (Okita et al., 2012; Kasahara et al., 2010). Since Chk1 is able to regulate pathways involved in both $\mathrm{G}_{1}$ and $\mathrm{G}_{2}$ arrest, it is 
not a great indicator of phase-specific arrest mechanisms. However, Chk2 activity provides a better discrimination of phase-specific arrest mechanisms as it is centrally focused on regulating $G_{1}$ phase cell cycle arrest. The activation of Chk2 can function to phosphorylate both Cdc25a and other downstream targets, such as tumor protein 53 (p53), which functions to activate stress responsive pathways and induce $G_{1}$ arrest (Levine and Oren, 2009; Chehab et al., 1999). Indeed previous research on anoxia effects on red-eared slider turtles indicated an activation of p53 in response to anoxia (Zhang et al., 2013). Interestingly, that study detailed the relative phosphorylation of p53 at Ser20 by Chk 2 which increased 2 to 3 -fold in response to $20 \mathrm{~h}$ anoxia. This suggests a possible mechanism for Chk2 to regulate both Cdc25a and p53 function during anoxia in the turtle.

\subsubsection{Regulation of Cdc25a and Cdc25c}

The binding of the regulatory Cyclin proteins to Cdks is essential in the formation of the kinase complex, which upon dephosphorylation by Cdc25a and Cdc25c phosphatases, fully activates the kinase activity of the Cyclin/Cdk complex (Bloom and Cross, 2007; Ohtani and Nevins, 1994). Members of the Cdc25 family play pivotal roles in cell cycle progression. Cdc25a has been shown to be a critical regulator of the $\mathrm{G}_{1} / \mathrm{S}$ transition of mammalian cells by regulating the activation of Cdk4, Cdk6 and Cdk2. Similarly, $\mathrm{Cdc} 25 \mathrm{c}$ is able to regulate the $\mathrm{G}_{2} / \mathrm{M}$ transition by activating the kinase activity of Cdc2. In response to both 5 and $20 \mathrm{~h}$ anoxia, both Cdc25a and Cdc25c significantly decreased in total protein expression in all tissues studied (Figure 3.6). Although the analysis of total protein expression does not necessary indicate changes in activity, it does 
suggest that $\mathrm{Cdc} 25$ function (and downstream activation of $\mathrm{Cdks}$ ) is not critical to anoxia survival.

\subsubsection{Regulation of cyclin dependent kinase inhibitors}

CKIs are typically considered to be the main components involved in arresting the cell cycle. These inhibitors are divided based upon the mechanisms of Cdk inhibition into two distinct groups, the INK4 family and the Cip/Kip family (Nakayama and Nakayama, 1998; Sherr and Roberts, 1999). I examined the protein expression of three well-studied CKIs including the INK4 inhibitor p16 and Cip/Kip inhibitors, p27 and p21, along with the mRNA expression of $\mathrm{p} 27$. The $\mathrm{p} 16$ protein binds specifically to the $\mathrm{G}_{1} \mathrm{Cdks}$ (Cdk4 and Cdk6) and inhibits Cdk association with D-type cyclins, promoting a $G_{1}$ arrest (Figure 3.1) (Cánepa et al., 2007). To assess the relative expression and activity of p16, total protein levels were assessed in response to anoxia, as well as the relative levels of a phosphorylation site specific to active p16 protein (Thr145). Total protein levels of p16 increased significantly during anoxia in both liver (after $5 \mathrm{~h}$ anoxia) and kidney (after 20 h anoxia); no changes were detected in white skeletal muscle (Figure 3.7). In kidney tissue, the relative change in active p-p16 (Thr145) increased similar to that of total p16 expression in response to anoxia. However, in liver, p-p16 (Thr145) phosphorylation levels increased dramatically by $\sim 8$-fold after $5 \mathrm{~h}$ of anoxia exposure and remained high at $\sim 4$-fold over control values after $20 \mathrm{~h}$ anoxia (Figure 3.7). This indicates that p16 may be highly active in response to anoxia in turtle liver and suggests that it can play a major role in regulating a possible $\mathrm{G}_{1}$ arrest mechanism. As p16 is known to target $\mathrm{Cdk} 4$ and Cdk6 exclusively, its activation during anoxia coupled with the decreased expression 
Cyclin D1 in the liver and kidney (see Chapter 2, Figure 2.7), provides good evidence that the $\mathrm{G}_{1}$ stage of the cell cycle is strongly targeted for suppression during anoxia in these turtle tissues. No significant changes in either Cyclin D1 or p16 protein levels were detected in white skeletal muscle; this is likely a reflection of the senescent nature of skeletal myocytes (after differentiation) and not of alternative mechanisms of cell cycle regulation (Walsh and Perlman, 1997).

The Cip/Kip proteins inhibit the enzymatic complex formed by the Cyclin and Cdk proteins via interactions with the catalytic subunit of Cdk (Figure 3.1) (Cayrol et al., 1998). The present results show that $\mathrm{p} 27$ protein and transcript expression displays tissue specific regulation; a significant increase in both parameters occurred after $20 \mathrm{~h}$ anoxia in liver tissue, a significant decrease in protein expression after $5 \mathrm{~h}$ and decrease in both protein and transcripts occurred after $20 \mathrm{~h}$ anoxia in kidney (Figure 3.7). The relative level of inhibitory phosphorylation of p27 (Thr187) decreased strongly in both liver and kidney tissue in response to 5 and $20 \mathrm{~h}$ anoxia. Interestingly, p27 is actively phosphorylated (at Thr187) and inhibited by the kinase action of Cyclin/Cdk complexes, such as Cyclin E1/Cdk2 and Cyclin A/Cdk2. A decrease in the relative phosphorylation level of p-p27 (Thr187) may be a result of a reduction in Cyclin/Cdk activity in response to anoxia or may be a result of the inactivation of Cdk proteins (Tyr14/Thr15; see

Chapter 2, Figure 2.14). Indeed multiple studies have found that a decrease in Thr187 phosphorylation of p27 is indicative of a strong cell cycle arrest (Roy et al., 2007; Besson et al., 2006). Taken together, these results suggest a role for $\mathrm{p} 27$-induced cell cycle regulation in during anoxia.

Several studies have indicated that the hypoxia-inducible factor 1a (HIF-1 $\alpha)$ plays 
an essential role in the adaptive response of cells to hypoxia (Aragones et al., 2009). Under hypoxic conditions, HIF-1 $\alpha$ has a well-known role in the reorganization of glycolysis and has recently been indicated in the cessation of cellular proliferation through the induction of CKIs. Studies have suggested that increases in HIF-1 $\alpha$ transactivation activity increase expression of p27 (Horree et al., 2008). Additional studies using HIF-1 $\alpha$ null murine embryonic fibroblasts identified p27 as having a role in hypoxia-induced cell-cycle arrest and that this expression is indeed HIF-1 $\alpha$ dependent (Dang et al., 2008). Although there are currently no studies documenting the role of HIF$1 \alpha$ in the liver of anoxic turtles, it could be hypothesized that a HIF- $1 \alpha / \mathrm{p} 27$ interaction may play a role in connecting the typical hypoxia response to the induction of cell cycle arrest and the reorganization of glycolysis.

In contrast to $\mathrm{p} 27$, total expression levels of $\mathrm{p} 21$ protein did not significantly increase in response to anoxia in either liver or kidney tissues; an increase was detected after $5 \mathrm{~h}$ anoxia in white skeletal muscle by decreased after $20 \mathrm{~h}$ of anoxia back to normoxic levels (Figure 3.7). Several kinases can phosphorylate p21at residue Thr145, preventing its inhibitory functions in cell cycle regulation. Such kinases include Akt (studied in Chapter 2) and Pim-1 (not studied in this thesis). The relative levels of p-p21 (Thr145) were found to significantly increase in response to both 5 and $20 \mathrm{~h}$ anoxia in liver and kidney tissue; a significant decrease after $20 \mathrm{~h}$ anoxia was found in white skeletal muscle. An increase in p-p21 (Thr145) phosphorylation levels prevents p21 protein from inhibiting the cell cycle by multiple mechanisms. This includes preventing the complex formation of $\mathrm{p} 21$ with proliferating cell nuclear antigen (PCNA), thereby inhibiting DNA replication. In addition, phosphorylation of $\mathrm{p} 21$ at Thr145 decreases the 
binding of both Cdk2 and Cdk4 to p21 and attenuates the Cdk inhibitory activity of $\mathrm{p} 21$. Taken together, these results suggest that $\mathrm{p} 21$ does not play a significant role in regulating the cell cycle in response to anoxia in the studied tissues of the turtle.

\subsubsection{Conclusion}

In conclusion the present study builds upon the possible regulatory mechanisms of the research findings previously presented in Chapter 2. Previous results from Chapter 2 indicated a general regulation of critical cell cycle components, namely Cyclins and Cdks, in addition to a possible regulation of a signaling cascade known to drive cell cycle progression. Although indicating a slowing or general arrest of the cell cycle, the study did not provide any information regarding phase-specific regulation. The goal of this chapter was to develop the findings of the previous study and detail possible cell cycle arrest mechanisms (those specific to either $\mathrm{G}_{0} / \mathrm{G}_{1}$ or $\mathrm{G}_{2}$ ) that might be involved in regulating the cell cycle during periods of anoxia exposure in the turtle. Overall, the results show a clear activation of proteins involved in establishing and maintaining $G_{0} / G_{1}$ arrest, including CKIs (p16 and p27) and Chk2 proteins. Mechanisms involved in establishing both $\mathrm{G}_{0} / \mathrm{G}_{1}$ and $\mathrm{G}_{2}$ arrest (Chk1 and $\left.\mathrm{p} 21\right)$ were not found to be activated in response to anoxia, further indicating a $\mathrm{G}_{0} / \mathrm{G}_{1}$ arrest mechanism. Although this study established that (1) cell cycle arrest mechanisms are upregulated in response to anoxia and (2) these arrest mechanisms are specific to $G_{0} / G_{1}$ cell cycle arrest, it is still unclear how the downstream regulators of $\mathrm{G}_{0} / \mathrm{G}_{1}$ arrest (Rb and $\mathrm{E} 2 \mathrm{~F}$ ) are regulated and whether arrest is specific to the $\mathrm{G}_{1}$ or $\mathrm{G}_{0}$ phases of the cell cycle. Studies next need to analyze the specific mechanisms of $\mathrm{G}_{0} / \mathrm{G}_{1}$ phase arrest of the cell cycle, exploring both the differential regulation and protein interactions that are markers of either type of cell cycle 
arrest. This is the topic of Chapter 4. In particular, these studies will examine the protein make-up of inhibitory transcriptional complexes built upon E2F family members since it is this transcriptional complex that directs the cell cycle to remain arrested in the $\mathrm{G}_{1}$ phase, or enter reversible quiescence $\left(\mathrm{G}_{0}\right)$. These studies in particular, will be needed to fully obtain a complete understanding of the regulatory mechanisms at play in anoxic $T$. scripta elegans. 


\section{References}

Abraham, R.T. 2001. Cell cycle checkpoint signaling through the ATM and ATR kinases. Gene Dev., 15(17), 2177-2196.

Aragones, J., Fraisl, P., Baes, M., and Carmeliet, P. 2009. Oxygen sensors at the crossroad of metabolism. C. Metabolism, 9, 11-22.

Bencokova, Z., Kaufmann, M., Pires, I., Lecane, P., Giaccia, A., and Hammond, E. 2009. ATM activation and signaling under hypoxia conditions. Mol. Cell. Biol., 29, 526-537.

Besson, A., Gurian-West, M., Chen, X., Kelly-Spratt, K.S., Kemp, C.J., and Roberts, J.M. 2006. A pathway in quiescent cells that controls p27KIP1 stabililty, subcellular localization, and tumor suppression. Genes \& Dev., 20, 47-64.

Bloom, J., and Cross, FR. 2007. Multiple levels of cyclin specificity in cell-cycle control. Nat. Rev. Mol. Cell Biol., 8(2), 149-160.

Cánepa, E.T., Scassa, M.E., Ceruti, J.M., Marazita, M.C., Carcagno, A.L., Sirkin, P.F., and Ogara, M.F. 2007. INK4 proteins, a family of mammalian CDK inhibitors with novel biological functions. IUBMB Life, 59(7), 419-426.

Cayrol, C., Knibiehler, M., and Ducommun, B. 1998. p21 binding to PCNA causes G1 and G2 cell cycle arrest in p53-deficient cells. Oncogene, 12(3), 311-320.

Chehab, N.H., Malikzay, A., Stravridi, E.S., and Halazonetis, T.D. 1999. Phosphorylation of Ser-20 mediates stabilization of human p53 in response to DNA damage. Proc. Natl. Acad. Sci. USA, 96, 13777-13782.

Choudhury, A., Cuddihy, A., and Bristow, R. 2007. Radiation and new molecular agents Part I: Target ATM-ATR checkpoints, DNA repair, and the proteasome. Seminars in Radiation Oncology, 16, 51-58.

Clegg, J.S. 1997. Embryos of Artemia franciscana survive four years of continuous anoxia: the case for complete metabolic rate depression. J. Exp. Biol., 200, 467475.

Coleman, K.G., Waulet, B.S., Morrissey, D., Mulheron, J., Sedman, S.A., Brinkley, P., Price, S., and Webster, K.R. 1997. Identification of CDK4 sequences involved in Cyclin D1 and p16 binding. J. Biol. Chem., 272, 18869-18874.

Dang, C., Kim, J., Gao, P., and Yustein, J. 2008. The interplay between MYC and HIF in cancer. Nat. Rev. Cancer, 8, 51-56.

Douglas, R., and Haddad, G. 2001. Cell cycle progression and cell division are sensitive to hypoxia in Drosophila melanogaster embryos. Am. J. Physiol. Regul. Integr. Comp. Physiol., 280(5), R1555-R1563.

Douglas, R., and Haddad, G. 2003. Effect of oxygen deprivation on cell cycle activity: a profile of delay and arrest. J. Appl. Physiol., 94, 2068-2083. 
Falck, J., Mailand, N., Syljuasen, R.G., Bartek, J., and Lukas, J. 2001. The ATM-Chk2Cdc25a checkpoint pathway guards against radioresistant DNA synthesis. Nature, $410,842-847$.

Guardavaccaro, D., and Pagano, M. 2006. Stabilizers and destabilizers controlling cell cycle oscillators. Mol. Cell, 22, 1-4.

Hammond, E.M., and Giaccia, A.J. 2004. The role of ATM and ATR in the cellular response to hypoxia and re-oxygenation. DNA Repair, 3, 1117-1122.

Hand, S.C. 1998. Quiescence in Artemia franciscana embryos: reversible arrest of metabolism and gene expression at low oxygen levels. J. Exp. Biol., 201, 12331242 .

Harper, J., Adami, G., Wei, N., Keyomarsi, K., and Elledge, S. 1993. The p21 cdkinteracting protein $\mathrm{Cip} 1$ is a potent inhibitor of $\mathrm{G}_{1}$ cyclin-dependent kinases. Cell, $75,805-816$.

Horree, N., Gort, E., Van der Groep, P., Heintz, A., Vooijs, M., and van Diest, P. 2008. Hypoxia-inducible factor 1 alpha is essential for hypoxic p27 induction in endometrioid endometrical carcinoma. J. Pathol., 214, 38-45.

Kasahara, K., Goto, H., Enomoto, M., Tomono, Y., Kiyono, T., and Inagaki, M. 2010. 14-3-3gamma mediates Cdc25A proteolysis to block premature mitotic entry after DNA damage. EMBO J., 29(16), 2802-12.

Kolaeva, S., Kramarova, L., Ilyasova, E., and Ilyasov, E. 1980. The kinetics and metabolism of the cells of hibernating animals during hibernation. Int. Rev. Cytol., 66, 147-170.

Kruman, I., Kolaeva, E., Iljasova, E., Zubrikhina, G., and Khachko, V. 1986. Seasonal variations of DNA synthesis in intestinal epithelial cells of hibernating animals I. DNA synthesis in intestinal epithelial cells of ground squirrel (Citellus undulatus) during deep hibernation. Comp. Biochem. Physiol., 83B, 173-177.

Kwon, Y.H., Jovanovic, A., Serfas, M.S., Kiyokawa, H., and Tyner, A.L. 2002. p21 functions to maintain quiescence of p27-deficient hepatocytes. J. Biol. Chem., 277, 41417-41422.

Levine, A.J., and Oren, M. 2009. The first 30 years of p53: growing ever more complex. Nat Rev Cancer, 9(10), 749-58.

Mazia, D. 1962. Biochemistry of the dividing cell. Annu. Rev. Biochem., 30, 669-688.

Muda, M., and McKenna, S. 2004. Model organisms and target discovery. Drug Discov. Today Tech., 1, 55-59.

Nakayama, K., and Nakayama, K. 1998. Cip/Kip cyclin-dependent kinase inhibitors: brakes of the cell cycle engine during development. Bioessays, 20(12), 10201029 .

Obaya, A., and Sedivy, J. 2002. Regulation of cyclin-cdk activity in mammalian cells. Cell Mol. Life Sci., 59, 126-142. 
Ohtani, K., and Nevins, J.R. 1994. Functional properties of a Drosophila homolog of the E2F1 gene. Mol. Cell. Biol., 14(3), 1603-1612.

Okita, N., Minato, S., Ohmi, E., Tanuma, S., and Higami, Y. 2012. DNA damageinduced CHK1 autophosphorylation at Ser296 is regulated by an intramolecular mechanism. FEBS Lett., 586(22), 3974-3979.

Padilla, P.A., and Roth, M.B. 2001. Oxygen deprivation causes suspended animation in the zebrafish embryo. Proc. Natl. Acad. Sci. USA, 98, 7331-7335.

Padilla, P.A., Nystul, T.G., Zager, R.A., Johnson, A.C., and Roth, M.B. 2002.

Dephosphorylation of cell cycle-reulated proteins correlates with anoxia-induced suspended animation in Caenorhabditis elegans. Mol. Biol. Cell, 13, 1473-1483.

Pavletich, N.P. 1999. Mechanisms of cyclin-dependent kinase regulation: Structures of Cdks, their Cyclin activators, and Cip and INK4 inhibitors. J. Mol. Biol., 287, 821-828.

Piret, B., Schoonbroodt, S., and Piette, J. 1999. The ATM protein is required for sustained activation of NF-kappaB following DNA damage. Oncogene, 18(13), 2261-2271.

Powers, J.T., Hong, S.K., Mayhew, C.N., Rogers, P.M., Knudsen, E.S., and Johnson, D.G. 2004. E2F1 uses the ATM signaling pathway to induce p53 and Chk2 phosphorylation and apoptosis. Mol. Cancer Res., 2, 203-214.

Roy, S., Kaur, M., Agarwal, C., Tecklenburg, M., Sclafani, R.A., and Agarwal, R. 2007. P21 and p27 induction by silibinin is essential for its cell cycle arrest effect in prostate carcinoma cells. Mol. Cancer Ther., 6, 2696-2707.

Schafer, K.A. 1998. The cell cycle: a review. Vet. Pathol., 35(6), 461-478.

Shibat, A., Barton, O., Noon, A., Dahm, K., Deckbar, D., Goodarz, A.A., Lobrich, M., and Jeggo, P.A. 2009. The maintenance of ATM dependent G2/M checkpoint arrest following exposure to ionizing radiation. Acta. Med. Nagasaki., 53, 19-21.

Sherr, C.J., and Roberts, J. 1995a. Inhibitors of mammalian $\mathrm{G}_{1}$ cyclin dependent kinase. Genes Dev., 9, 1149-1163.

Sherr, C.J., and Roberts, J. 1995b. CDK inhibitors: positive and negative regulators of $\mathrm{G}_{1}$-phase progression. Genes Dev., 13, 1504-1512.

Sherr, C.J., and Roberts, J.M. 1999. CDK inhibitors: positive and negative regulators of G1-phase progression. Genes Dev., 13(12), 1501-12.

Toyoshima, H., and Hunter, T. 1994. p27, a novel inhibitor of $\mathrm{G}_{1}$ cyclin-cdk protein kinase activity, is related to p21. Cell, 78, 67-74.

Walsh, K., and Perlman, H. 1997. Cell cycle exit upon myogenic differentiation. Curr. Opin. Genet. Dev. 7(5), 597-602.

Zhang, J., Biggar, K.K., and Storey, K.B. 2013. Regulation of p53 by reversible posttranscriptional and post-translational mechanisms in liver and skeletal muscle of an anoxia tolerant turtle, Trachemys scripta elegans. Gene, 513(1), 147-155. 


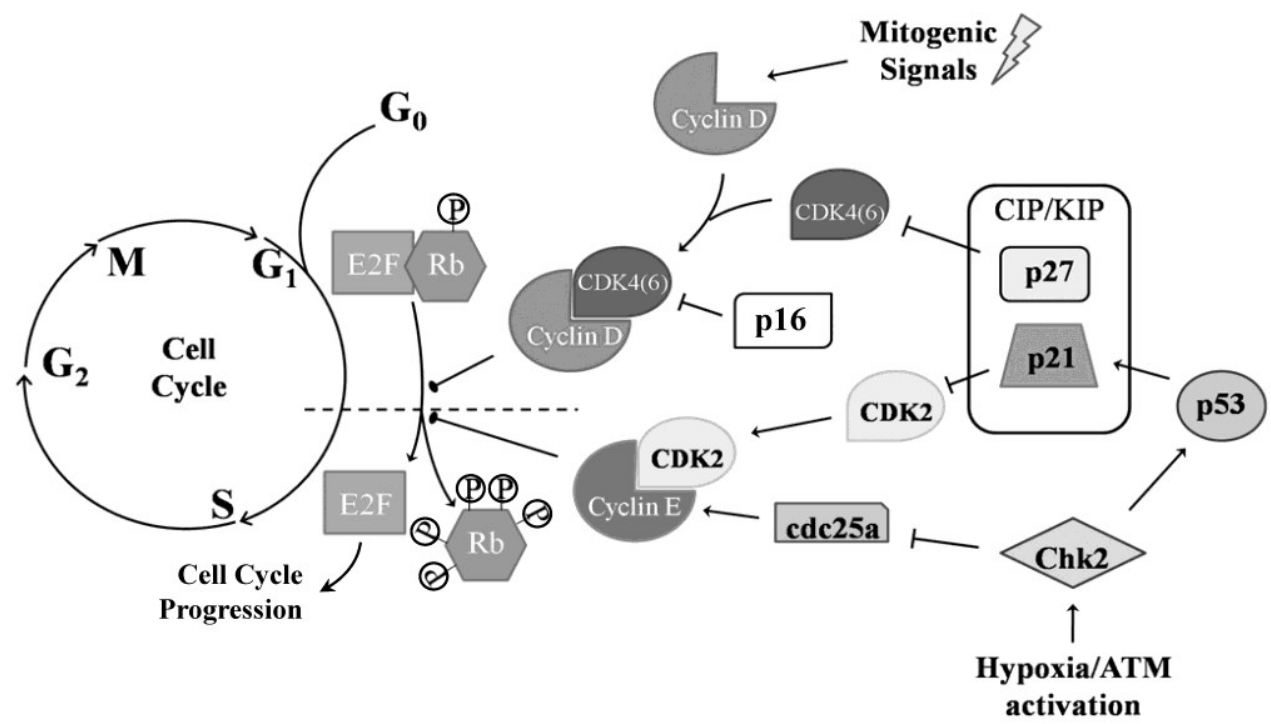

Figure 3.1. Proposed hypoxia induced regulation of the cell cycle. 


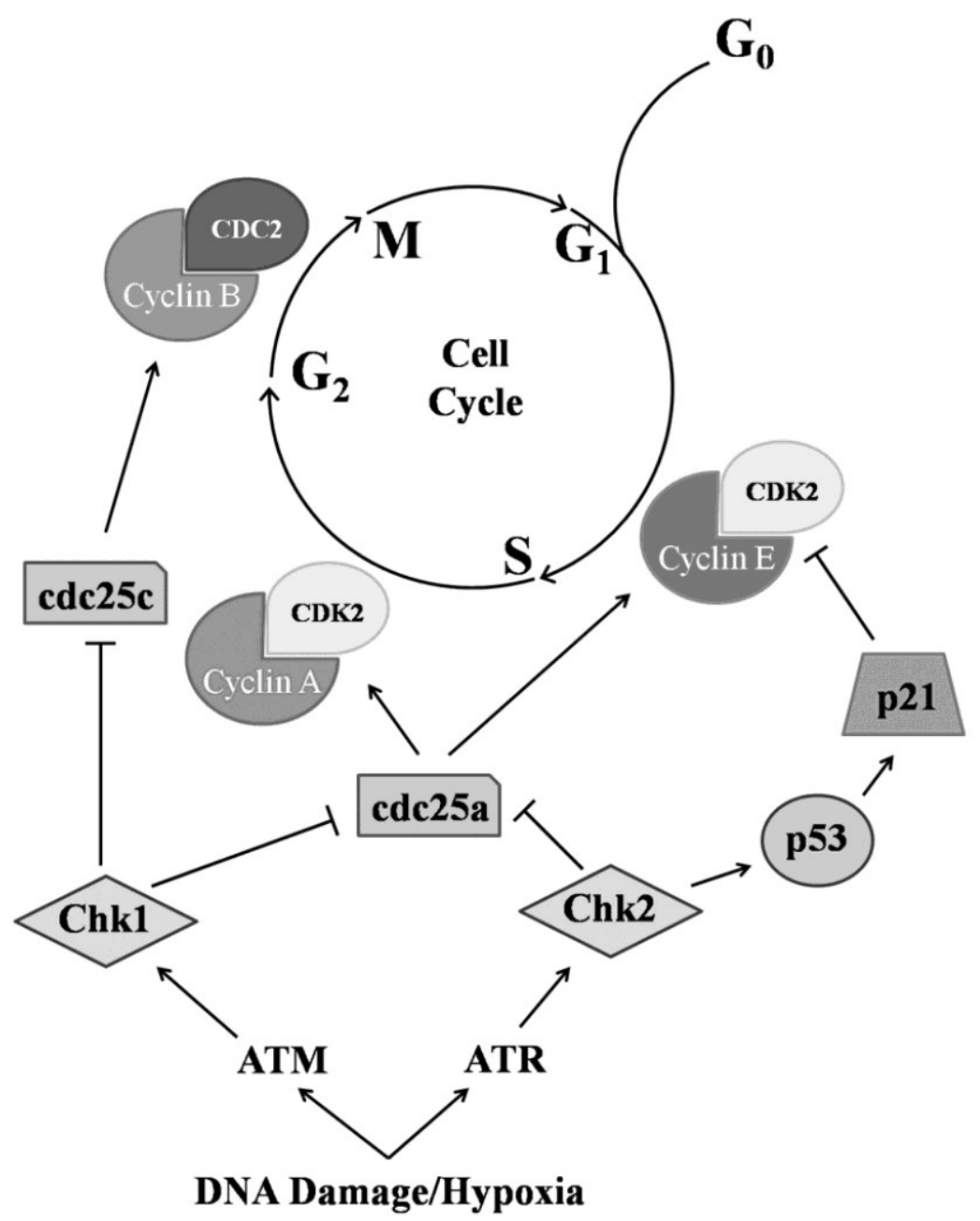

Figure 3.2. The ATM/ATR pathway. Stress activation of either ATM and ATR results in downstream phosphorylation of checkpoint kinases $(\mathrm{Chk} 1 / 2)$ and regulation of cell cycle effectors. 

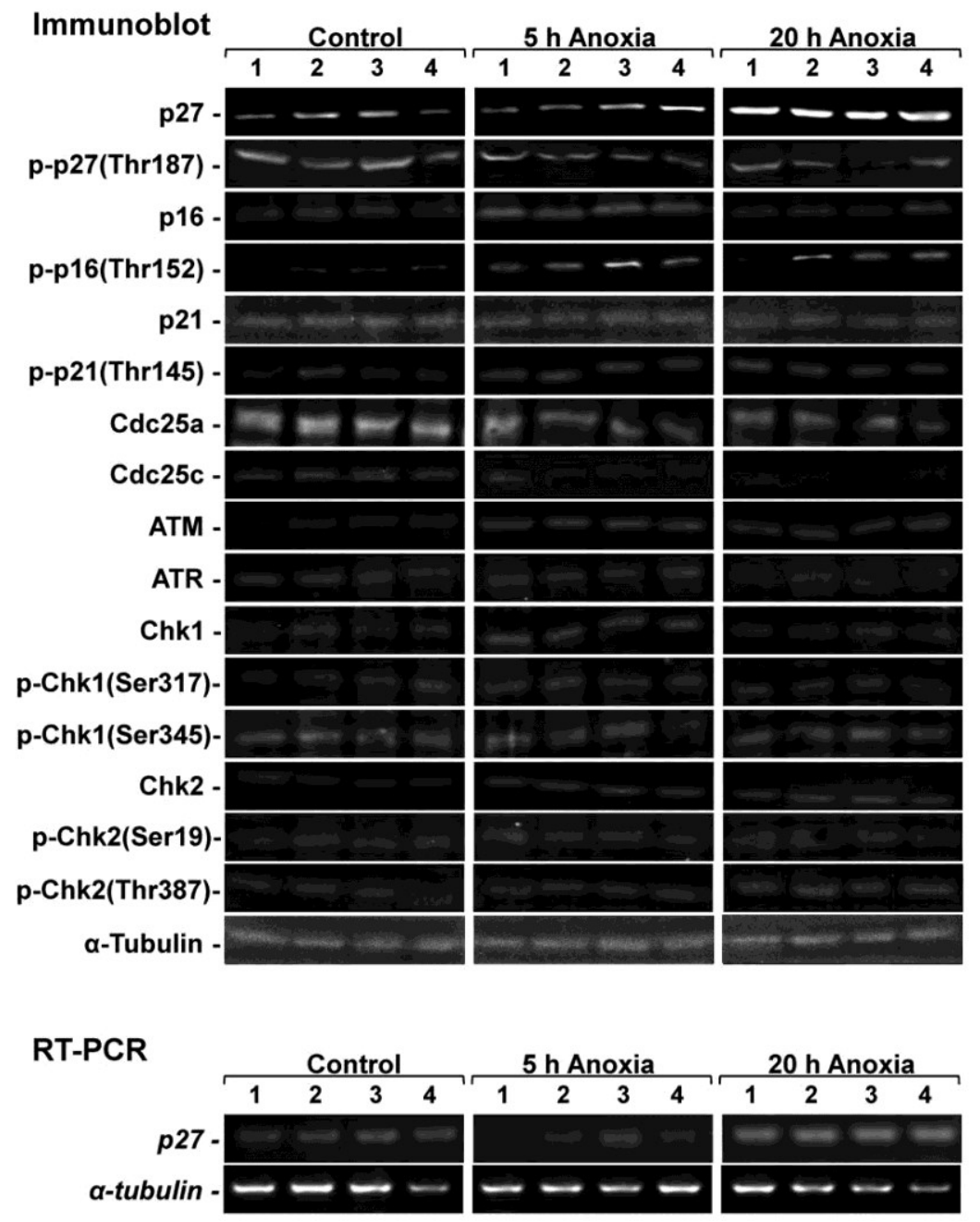

Figure 3.3A Image files for immunoblotting and RT-PCR targets in control and anoxic (5 and $20 \mathrm{~h}$ ) liver tissue from T. scripta elegans. 

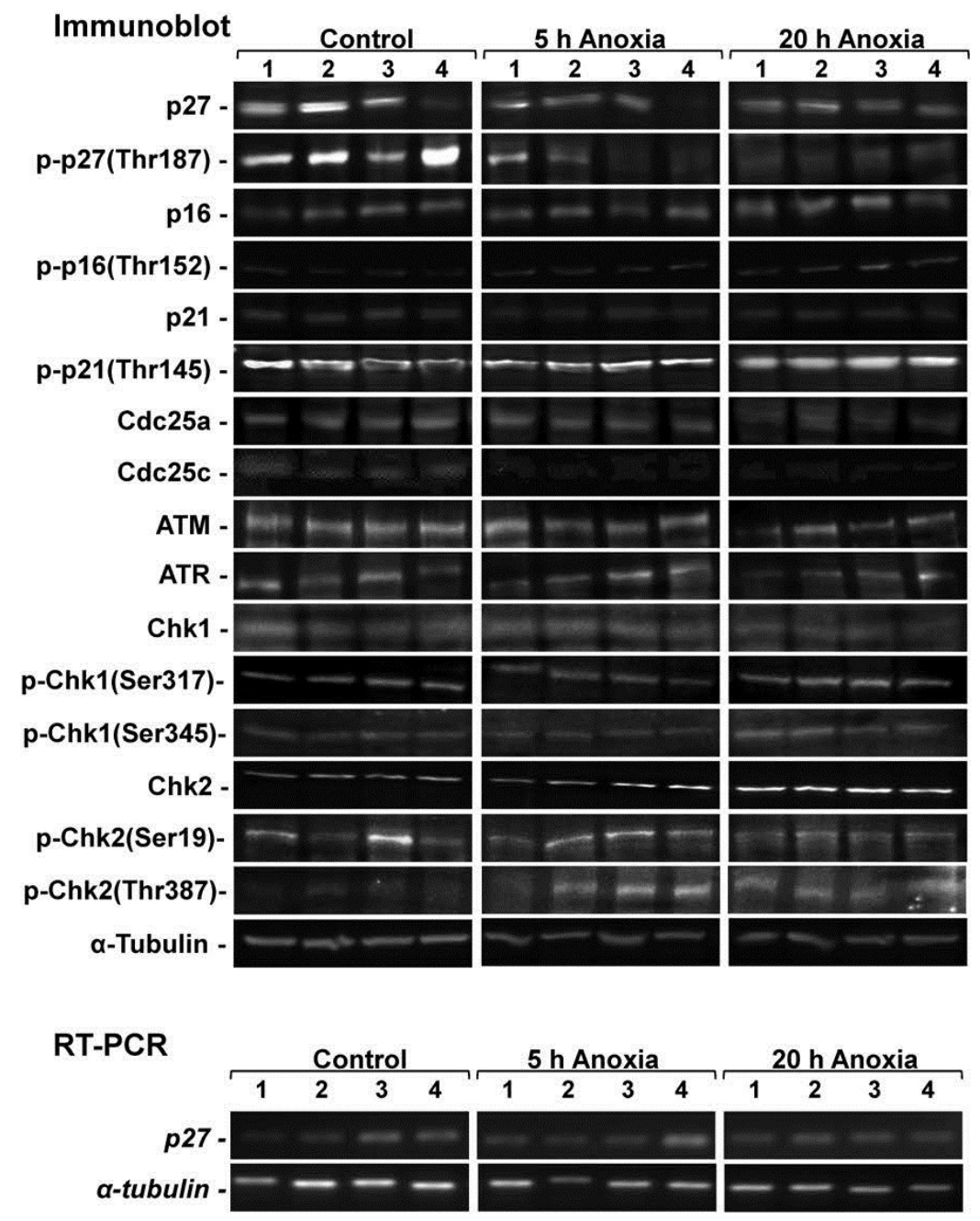

Figure 3.3B Image files for immunoblotting and RT-PCR targets in control and anoxic (5 and $20 \mathrm{~h}$ ) kidney tissue from $T$. scripta elegans. 

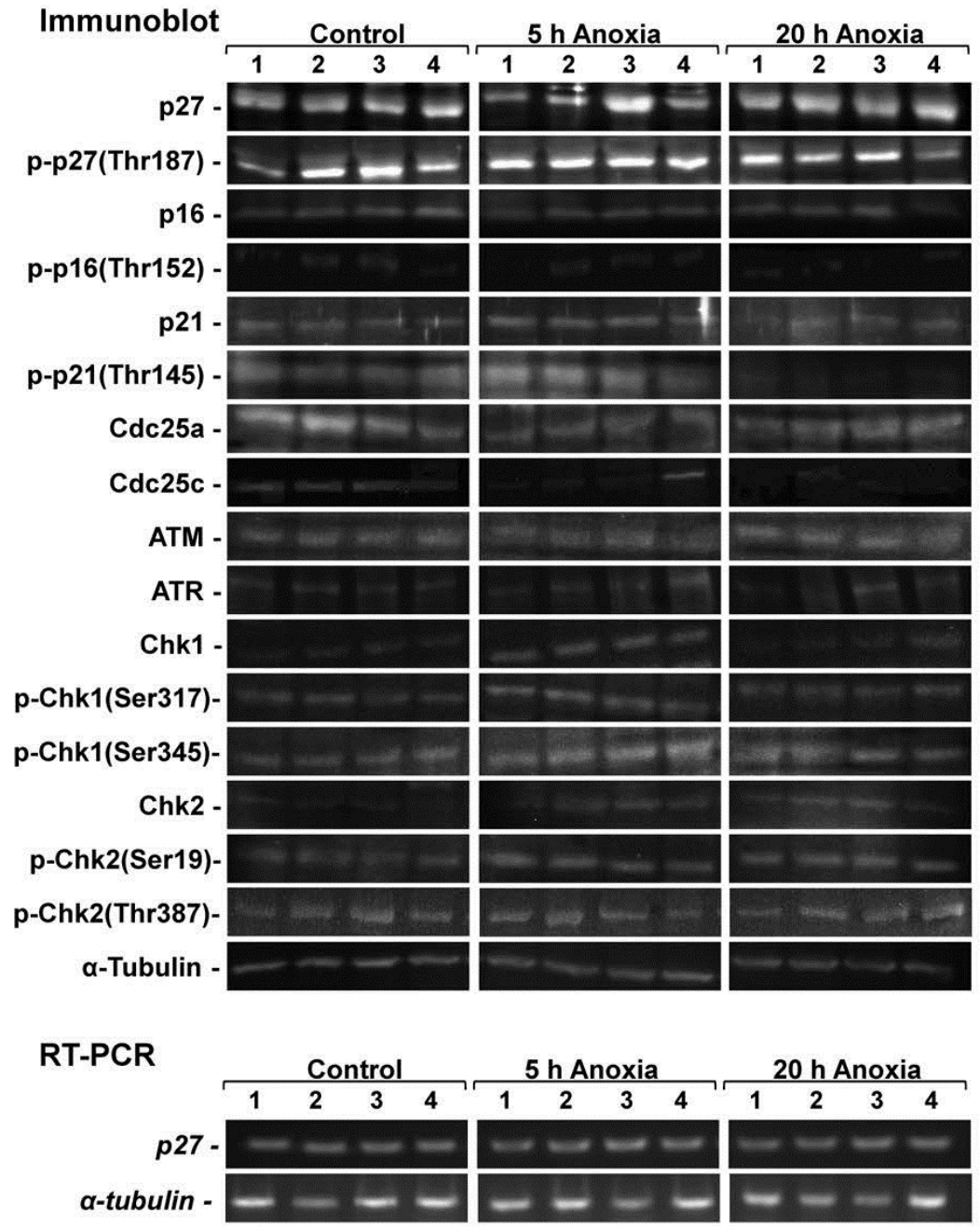

Figure 3.3C Image files for immunoblotting and RT-PCR targets in control and anoxic (5 and $20 \mathrm{~h}$ ) white skeletal muscle tissue from T. scripta elegans. 
A)

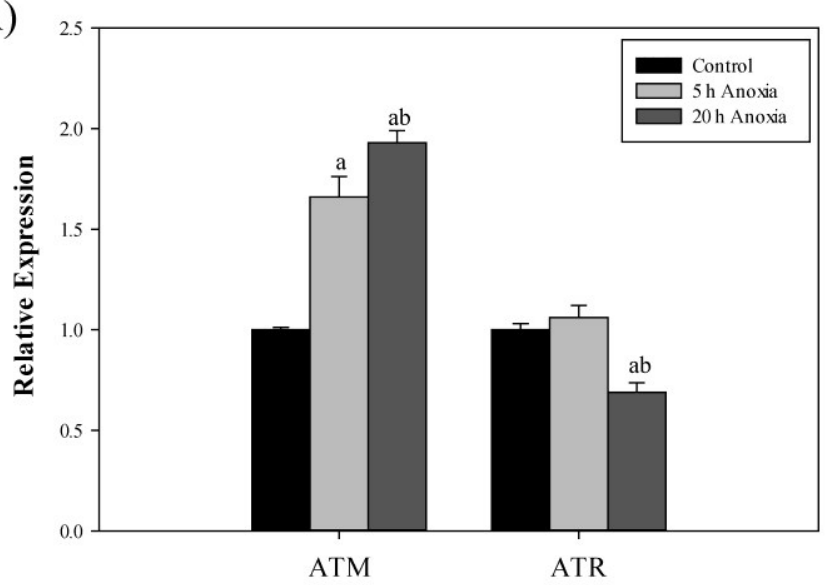

B)

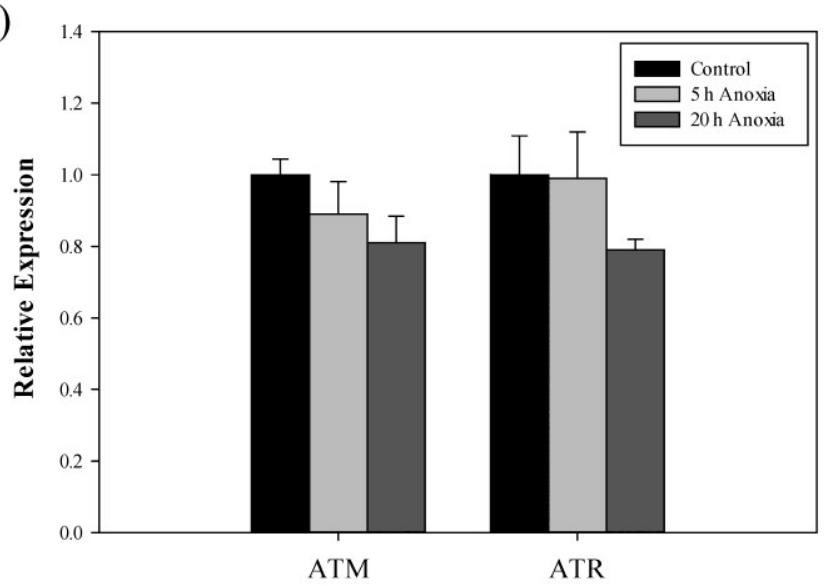

C)

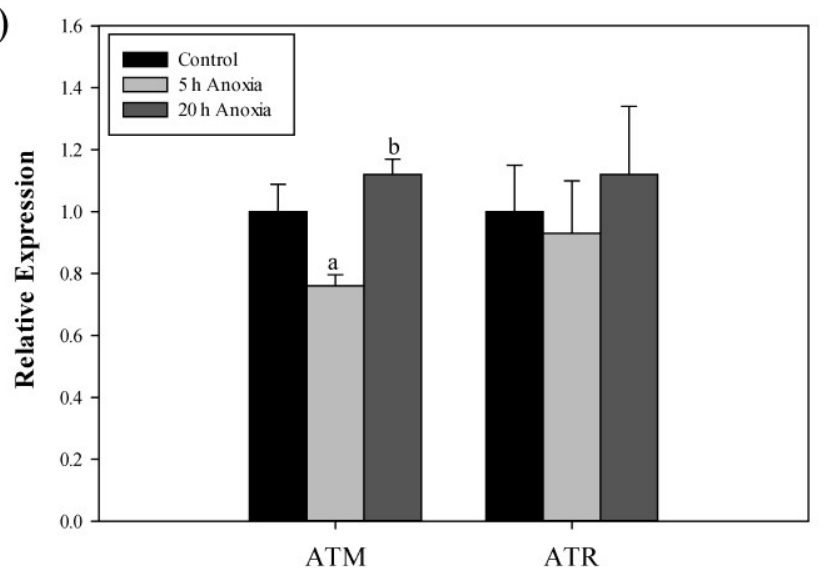

Figure 3.4. Effect of 5 and $20 \mathrm{~h}$ of anoxic submergence on relative protein expression of ATM and ATR in T. s. elegans. Histograms show normalized expression levels under control and anoxic conditions in (A) liver, (B) kidney and (C) white skeletal muscle tissues. Data are means \pm S.E.M., $n=3-4$ independent trials. a: Significantly different from the corresponding control $(\mathrm{P}<0.05)$; b: significantly different from the $5 \mathrm{~h}$ anoxic value $(\mathrm{P}<0.05)$ 
A)

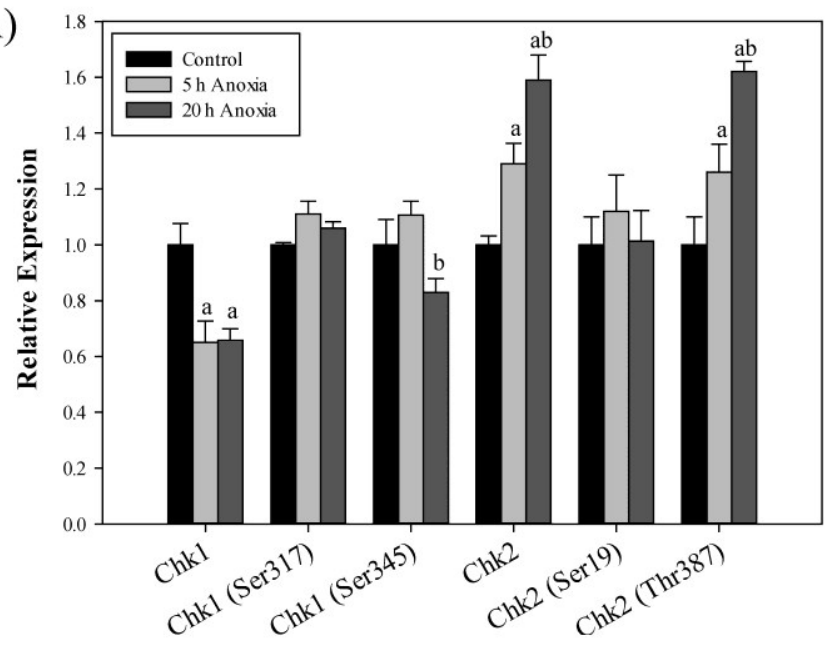

B)
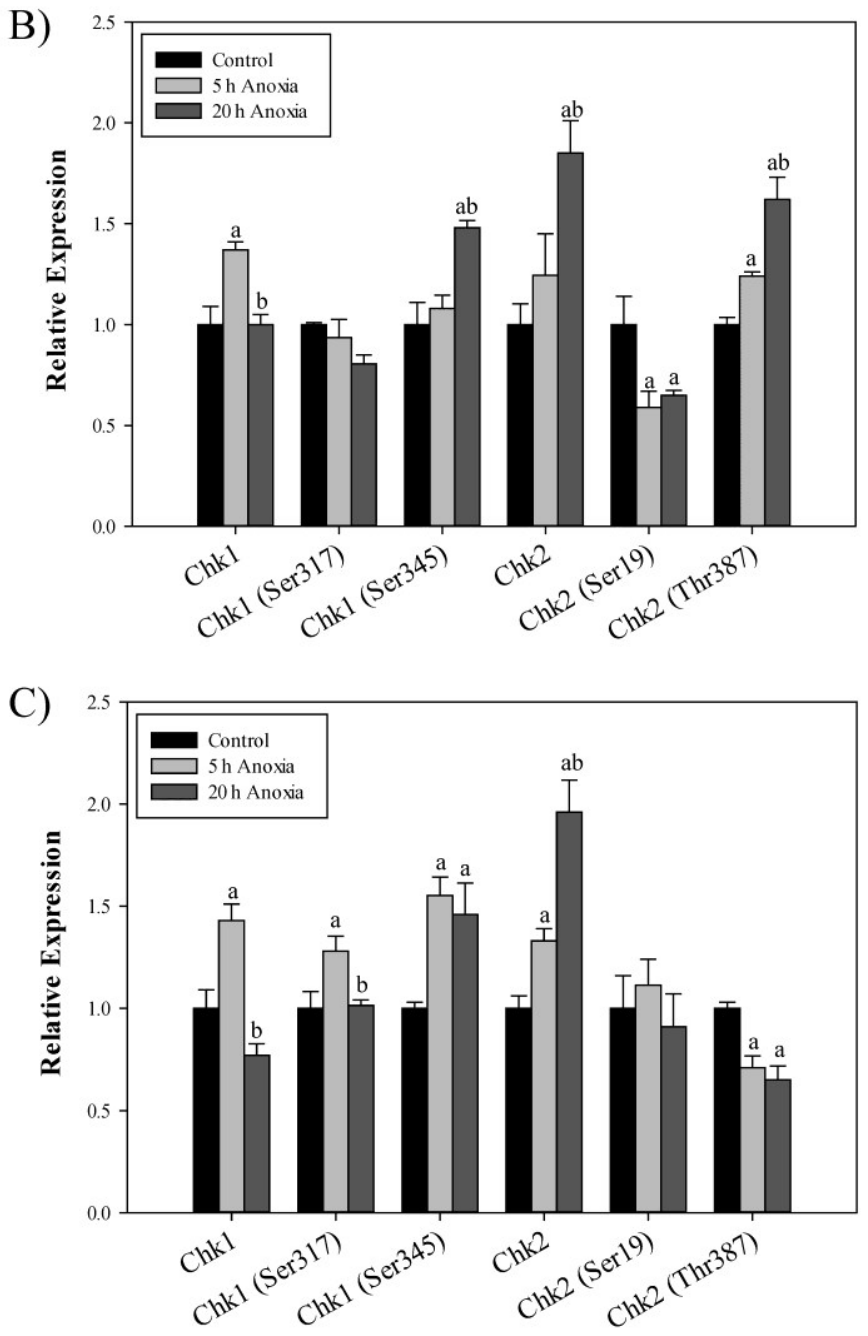

Figure 3.5. Effect of 5 and $20 \mathrm{~h}$ of anoxic submergence on relative protein and phosphorylation levels of Chk1 and Chk2 in T. s. elegans. Histograms show normalized expression levels under control and anoxic conditions in (A) liver, (B) kidney and (C) white skeletal muscle tissues. Data are means \pm S.E.M., $\mathrm{n}=3$ 4 independent trials. a: Significantly different from the corresponding control $(\mathrm{P}<0.05)$; b: significantly different from the $5 \mathrm{~h}$ anoxic value $(\mathrm{P}<0.05)$. 


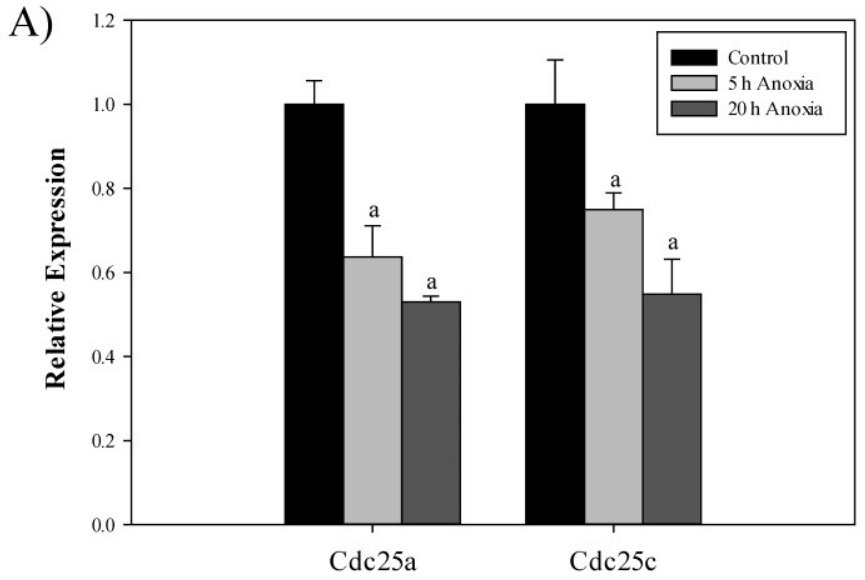

B)
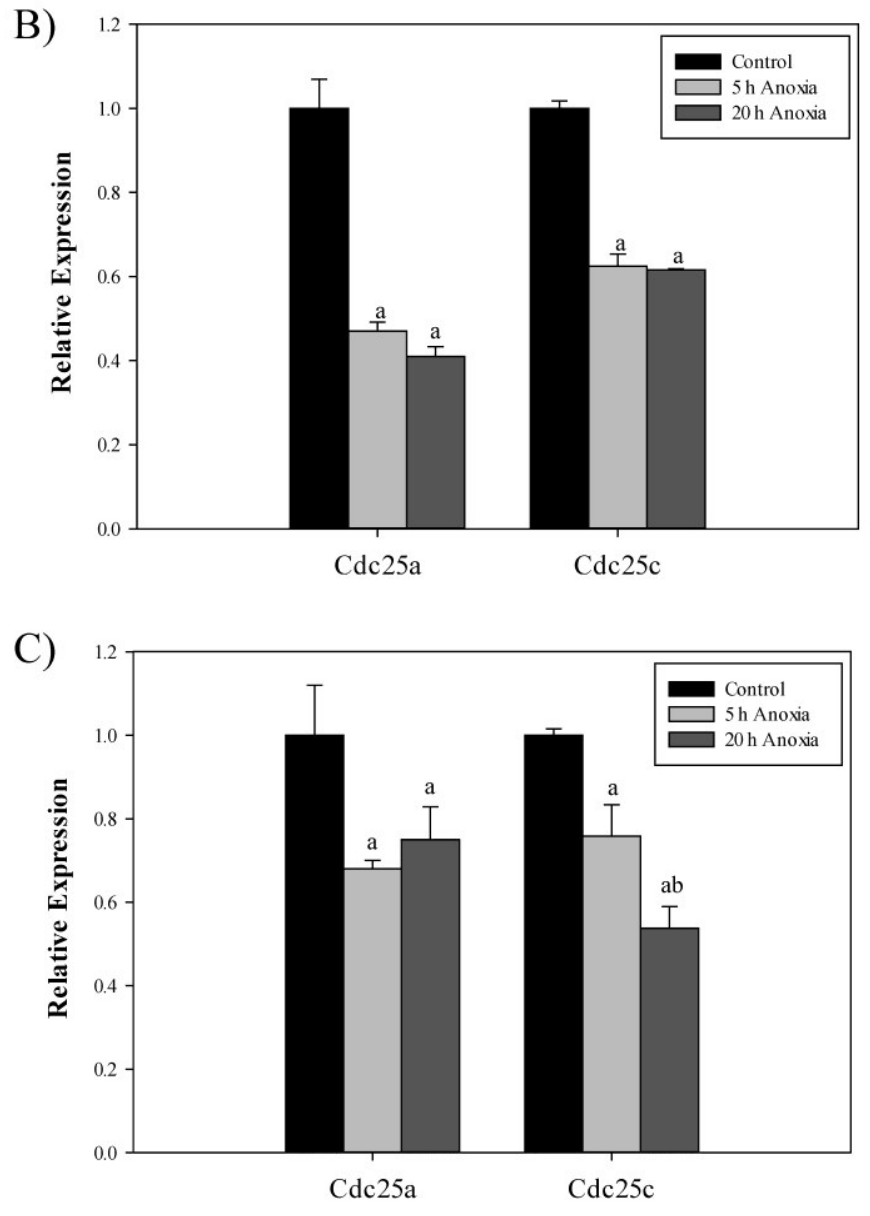

Figure 3.6. Effect of 5 and $20 \mathrm{~h}$ of anoxic submergence on relative protein expression of Cdc $25 \mathrm{a}$ and $\mathrm{Cdc} 25 \mathrm{c}$ in T. s. elegans. Histograms show normalized expression levels under control and anoxic conditions in (A) liver, (B) kidney and (C) white skeletal muscle tissues. Data are means \pm S.E.M., $\mathrm{n}=3$ 4 independent trials. a: Significantly different from the corresponding control $(\mathrm{P}<0.05)$; b: significantly different from the $5 \mathrm{~h}$ anoxic value $(\mathrm{P}<0.05)$. 

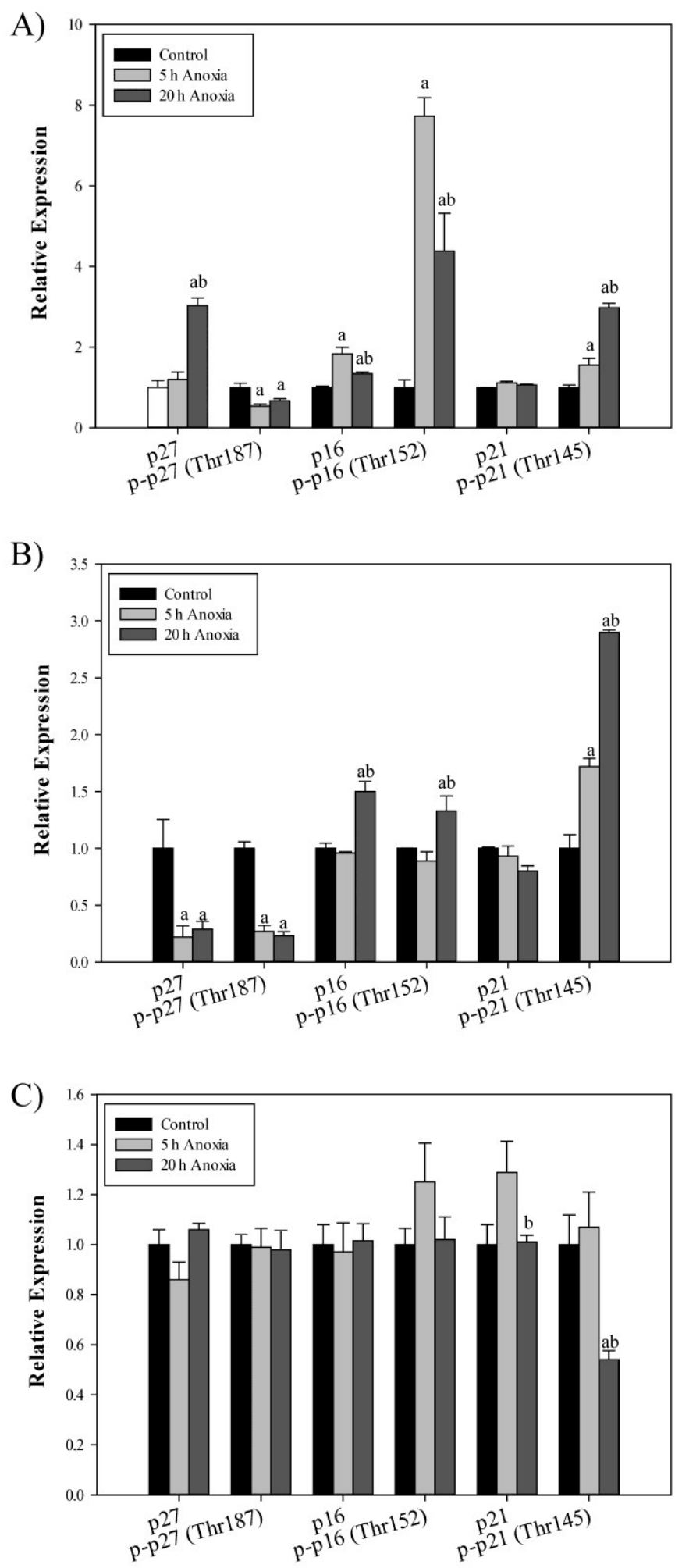

Figure 3.7. Effect of 5 and $20 \mathrm{~h}$ of anoxic submergence on relative protein and phosphorylation levels of CKIs (types p27, p16 and p21) in T. s. elegans. Histograms show normalized expression levels under control and anoxic conditions in (A) liver, (B) kidney and (C) white skeletal muscle tissues. Data are means \pm S.E.M., $n=3-4$ independent trials. a: Significantly different from the corresponding control $(\mathrm{P}<$ $0.05)$; b: significantly different from the $5 \mathrm{~h}$ anoxic value $(\mathrm{P}<0.05)$. 


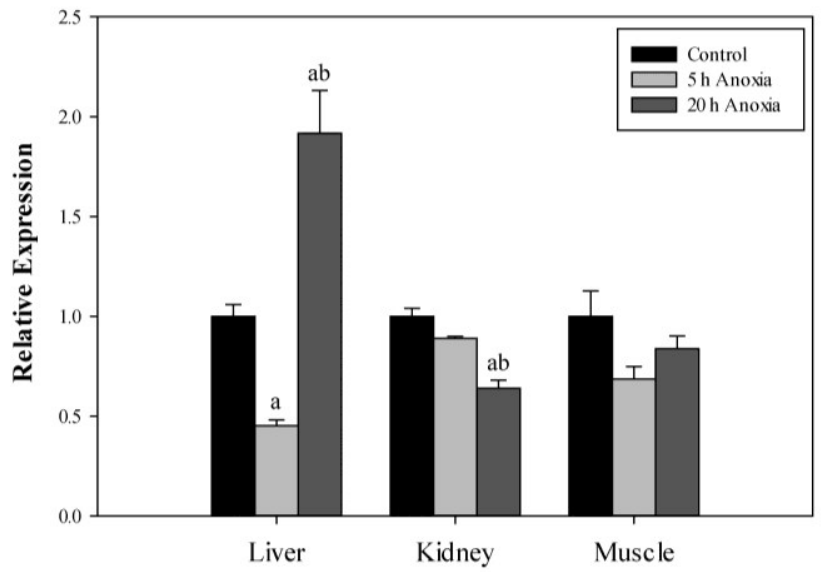

Figure 3.8. Effect of 5 and $20 \mathrm{~h}$ of anoxic submergence on transcript levels of $\mathrm{p} 27$ in T. s. elegans. Histograms show normalized expression levels under control and anoxic conditions in (A) liver, (B) kidney and $(C)$ white skeletal muscle tissues. Data are means \pm S.E.M., $n=3-4$ independent trials. a: Significantly different from the corresponding control $(\mathrm{P}<0.05)$; b: significantly different from the $5 \mathrm{~h}$ anoxic value $(\mathrm{P}<0.05)$. 
A)

1 TCTATCTAGCTGTCACGATGGATTTGATTTCCAGAATCACAAGCCCCTGGCTGGCAAGT

61 TCGAGTGGCAAGCCGTGGAGAAAGGGAGCTCGCCCGACTTCTACTTCAGACTCCCGAGGC

$\begin{array}{llllllllllllllllllllll}20 & F & E & W & Q & A & V & E & K & G & S & S & P & D & F & Y & F & R & L & P & R\end{array}$

121 CCCCCAAAGTCGCTTGCAAATCTGCCATCCATGAGAGTTTGGATGTAAATGGAAACTGCC

$\begin{array}{llllllllllllllllllllll}40 & \text { P } & \text { P } & \text { K } & \text { V } & \text { A } & \text { C } & \text { K } & \text { S } & \text { A } & \text { I } & \text { H } & \text { E } & \text { S } & \text { L } & \text { D } & \text { V } & \text { N } & \text { G } & \text { N } & \text { C }\end{array}$

181 CAACGGTGATTGTAGTCGGTTCTCAGGGAATCTCAGAGGACACTCACTTTGTAGATCAAA

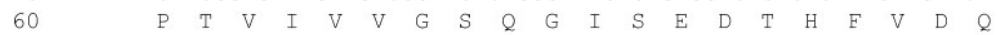

241 AAACTGATGTATCTGAAAATCAAACGGACTTAGCAGACCAGTGCAGTGGGCAAAGAAAAC

$\begin{array}{llllllllllllllllllllll}80 & K & T & D & V & S & E & N & \& & T & D & L & A & D & \& & C & S & G & \& & R & K\end{array}$

301 GACCAGCGACGGATGATTCTTCTCCTCAACCTAAAAGAGCCAACACAACAG

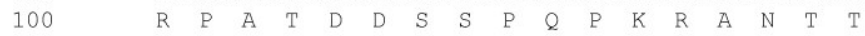

B)

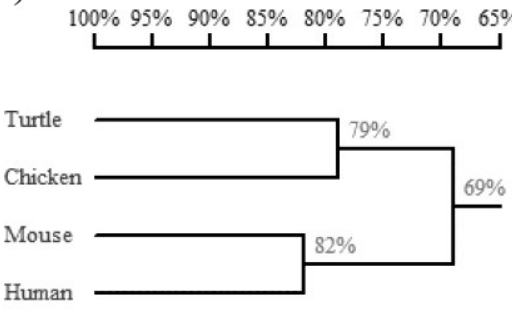

C)
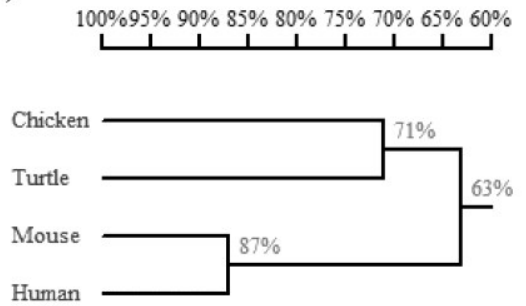

Figure 3.9. Nucleotide and deduced amino acid sequence for $T$. s. elegans partial $p 27$ sequence. Nucleotides and amino acids are numbered on the left. (A) Nucleotides and amino acids are numbered on the left. The nucleotide sequence was 332 nucleotides in length, while the amino acid sequence was 117 amino acids in length. Homology of (B) nucleotide and (C) protein sequences of the turtle to that of chicken (G. gallus), Mouse (M. musculus) and human (H. sapiens). 


\section{CHAPTER 4}

\section{REGULATION OF THE Rb/E2F TRANSCRIPTION FACTOR COMPLEX}




\subsection{Introduction}

The retinoblastoma $(\mathrm{Rb})$ gene was the first tumor suppressor to be discovered and since then has become a well-established member of cell cycle control (Delston and Harbour, 2006; Lundberg and Weinberg, 1999). First identified in its mutant form in the rare eye tumor, retinoblastoma, the $\mathrm{Rb}$ gene is known to be inactivated in nearly all human cancers, signifying its importance in the cell cycle and the regulation of proliferation (Delston and Harbour, 2006; Lundberg and Weinberg, 1999; Du and Pogoriler, 2006). The Rb family members (mainly Rb, and to a lesser extent p130 and p107) were initially envisioned as simple “on-off" switches that regulated the progression of the cell cycle. However, recent studies have revealed a complex set of Rb-protein interactions that add to the importance of these proteins in cell cycle regulation (Delston and Harbour, 2006). As a result, the Rb family has been shown to have a specialized role in cell cycle exit leading to senescence and quiescence, as well as the ability to block apoptosis. These characteristics make the $\mathrm{Rb}$ family of proteins an intriguing target for

analysis in cell cycle regulation of facultative anaerobiosis in which cells must reversibly inhibit mitosis to conserve energy stores.

\subsubsection{Retinoblastoma and cell cycle arrest}

Cellular proliferation involves the inactivation of at least one member of the $\mathrm{Rb}$ family, thereby leading to differential binding properties as well as the creation of specific phosphorylation markers that characterize the $\mathrm{G}_{1} / \mathrm{S}$ transition (Kops et al., 2002). The general mechanism by which the $\mathrm{Rb}$ protein family exerts its effects is through regulatory binding of the E2F protein family, inhibiting E2F-mediated transcription of 
cell cycle dependent genes (Bracken et al., 2004; Grana et al., 1998). Phosphorylated Rb is present at relatively constant levels throughout the cell cycle; however, at the $G_{1} / S$ transition point it is sequentially phosphorylated by Cyclin D1/Cdk4, Cyclin D1/Cdk6 and Cyclin E1/Cdk2 complexes, respectively, leading to a release of E2F and allowing cell cycle progression (Delston and Harbour, 2006; Schmitz et al., 2006; DeGregori, 2004).

Unlike $\mathrm{Rb}$, which remains at relatively constant protein levels, other $\mathrm{Rb}$ family members, $\mathrm{p} 107$ and $\mathrm{p} 130$, are dynamically regulated at both the protein and phosphorylation levels (Kops et al., 2002; Guardavaccaro and Pagano, 2006). Protein levels of p107 are maintained at low levels during quiescence and are expressed at higher levels during $\mathrm{G}_{1}$ phase, parallel to that of $\mathrm{Rb}$ expression (Guardavaccaro and Pagano, 2006; Grana et al., 1998). In an expression pattern opposing that of $\mathrm{p} 107, \mathrm{p} 130$ is maintained at lower levels in cycling cells and increases dramatically only during quiescence (Guardavaccaro and Pagano, 2006; Grana et al., 1998). The rise in p130 protein and mRNA levels specific to the quiescence stage of the cell cycle, is accompanied by an increase in the DNA-binding activity of E2F4 and repression of genes required for re-entry into the early $\mathrm{G}_{1}$ phase.

Phosphorylation of $\mathrm{Rb}$ in the late $\mathrm{G}_{1}$ phase regulates passage through the R-point transition, committing cells to mitosis (Guardavaccaro and Pagano, 2006). By contrast, hypophosphorylated $\mathrm{Rb}$ represses $\mathrm{E} 2 \mathrm{~F}$ family members 1 through 5 by binding the E2F transactivation domain and through the recruitment of chromatin remodeling complexes (Schmitz et al., 2006). The multiple protein interactions of $\mathrm{Rb}$ are largely controlled by serine and threonine phosphorylation (Leng et al., 2002; Hansen et al., 2001). Cycles of 
phosphorylation and dephosphorylation of Rb dominate cycling cells, however, cells undergoing quiescence harbor p130/E2F4 as the main pocket protein complex (Du and Pogoriler, 2006; Hansen et al., 2001). This complex mediates the gene repression of cell cycle regulators including E2F1 though E2F3, Cdc2 as well as type E1 and A1 Cyclins (Hansen et al., 2001). In addition to phospho-regulation of the Rb family, control through p300-dependant acetylation has been reported and is thought to regulate the $G_{1} / S$ transition by inhibiting kinase binding and Rb phosphorylation (Wong and Weber, 2007). Thus, $\mathrm{Rb}$ acetylation (Lys873) may also act as an important regulatory control in addition to phosphorylation, however, there has been limited research on the subject.

Interest in the E2F family of transcription factors increased dramatically when it was discovered that E2F proteins complexed with $\mathrm{Rb}$ (Trimarchi and Lees, 2002). Knowledge that E2F regulated the cell cycle provided a mechanism by which Rb could act to repress cellular proliferation (Bernards, 1997). The E2F family is comprised of five primary members, E2F1, E2F2 and E2F3 that interact exclusively with Rb and p107, as well as E2F4 and E2F5 which interact with Rb and p130 (Bernards, 1997). On the basis of sequence homology and functional properties, E2F1 through E2F3 are potent transcriptional activators and will be referred to as 'activating' E2F. By contrast, E2F4 and E2F5 are primarily involved in active repression of E2F mediated genes by recruiting p130 and associated chromatin remodeling complexes; these E2Fs will appropriately be referred to as 'repressive' E2F throughout the remainder of this thesis (Trimarchi and Lees, 2002).

Cells rely on several main pathways to regulate and maintain a quiescent state. These pathways converge on the regulation of p130 and CKIs. The Rb family member, 
p130, recruits the repressive E2F4 to appropriate promoter regions and facilitates binding to chromatin-remodeling complexes (Alvaro-Blanco et al., 2009). This chromatin remodeling blocks the transcription of many positive regulators of the cell cycle. The maintenance of quiescence is self-sustaining in the absence of mitogenic stimuli. These stimuli eventually lead to the active phosphorylation of Cdks and the decrease of CKIs (Guardavaccaro and Pagano, 2006; Litovchick et al., 2004).

\subsubsection{Heterochromatin regulation in response to cell cycle arrest}

Heterochromatin is composed of genomic DNA tightly packed by histones and non-histone proteins (Lu and Gilbert, 2007; Ferreira et al., 2001). Dynamic changes to chromatin structure prevent the access of transcription factors, such as E2F, to nucleosomal DNA. At least two primary mechanisms can be used to remodel chromatin structure. One mechanism involves changing the location and conformation of the nucleosomes through the use of ATP-dependent protein complexes such as the SWItch/Sucrose Non Fermentable complex (SWI/SNF) (Zhang and Dean, 2001; Kingston and Narlikar, 1999; Muchardt and Yaniv, 1999; Tyler and Kadonaga, 1999; van den Heuvel and Dyson, 2008). The second mechanism involves covalent modifications of histone N-terminal tails that protrude from the chromatin structure (Ferreira et al., 2001; Trojer et al., 2007). Studies examining the role of Rb/E2F mediated cell cycle arrest have identified key $\mathrm{Rb}$ interactions with chromatin remodeling factors (Giacinti and Giordano, 2006). The result of chromatin remodeling includes the effective repression of $\mathrm{E} 2 \mathrm{~F}$ transcriptional activity. Importantly, the particular associations between $\mathrm{Rb}$ and chromatin remodeling factors have been found to be dependent on the type of cell cycle 
exit (Lu and Gilbert, 2007).

General mechanisms of $\mathrm{Rb}$-mediated chromatin remodeling include the recruitment of ATP-dependent chromatin remodeling complexes. One particular complex member associated with $\mathrm{Rb}$ during cell cycle arrest is the SWI/SNF complex and its central subunit, Brahma (BRM) (Trotter and Archer, 2008; Shen, 2002). These chromatin remodeling complexes use the energy derived from ATP hydrolysis to alter the chromatin structure. The presence of the conserved Rb-binding motif, LXCXE, in BRM suggests that this may be the initial chromatin remodeling factor which binds to the Rb pocket domain, and prepares the nucleosomes for heterochromatin formation (Trotter and Archer, 2008).

Following BRG binding and nucleosome sliding, stable repression of $\mathrm{Rb} / \mathrm{E} 2 \mathrm{~F}$ is achieved through covalent modifications of protruding histone N-terminal tails (Shen, 2002). Deacetylation of histones is mediated through the recruitment of histone deacetylases (such as HDAC4) via the Rb associated protein RbAp48. The primary site of deacetylation during cell cycle exit is the acetylated lysine 9 on histone 3 (H3K9Ac), which later becomes methylated as well as a site of HP1 binding (Narita et al., 2003). Following H3K9Ac deacetylation, the next set of covalent histone modifications is methylation of lysine residues by histone methyltransferase (HMTase). The HMTase, Suv39H1, binds to $\mathrm{Rb}$ and more specifically, methylates lysine 9 of histone $\mathrm{H} 3$ (H3K9me) (van den Heuval and Dyson, 2008; Shen, 2002; Narita et al., 2003). Transcriptional repression of $\mathrm{E} 2 \mathrm{~F}$ through heterochromatin formation is also mediated through the binding of HP1 to multiple methylated H3K9 sites (Shen, 2002). Binding of HP1 mediates higher levels nucleosome structure through HP1 dimerization leading to 
multiple histone recruitment and tight chromatin packing (Fischle et al., 2005). Although these chromatin modifications are well cited in the literature during general exit and pausing of the cell cycle, there are slight modifications to these complexes in terminally differentiated cells (senescent) and those entering a prolonged reversible exit (quiescent) (Trojer and Kadonaga, 1999).

Quiescent transitions are controlled in a reversible manner, mediated in part by the competing actions of histone acetyltransferases (HATs) and HDACs on the histones associated with the $\mathrm{Rb} / \mathrm{E} 2 \mathrm{~F}$ complex. Although the general model of chromatin remodeling complexes have been characterized in cell cycle arrest, specific complexes characterizing prolonged and reversible cell cycle exit are only beginning to be explored. Chromatin modifying factors that have been found to interact with p130 during quiescence include components of the Drosophila Rb/E2F and Myb (LINC) complex, initially discovered in arrested Drosophila embryo extracts, and the histone lock (L3MBTL1) (Trojer and Kadonaga, 1999; Lewis et al., 2004). These complexes contain additional quiescent-specific factors which are thought to regulate quiescence by compacting nucleosome structures in a manner that is dependent on mono- and dimethylation of histone H3K9 (van den Heuval and Dyson, 2008). Recruitment of histone modifying proteins mediated by the LINC complex allows the binding of at least two nucleosomes simultaneously through the recognition of $\mathrm{N}$-terminal histone modifications (van den Heuval and Dyson, 2008). This raises the possibility that the LINC complex may promote specific and reversible heterochromatin structure through the recruitment of histone modifying proteins, thereby locking DNA in a condensed state (Trojer and Kadonaga, 1999). 
Although not all the proteins within the invertebrate LINC complex have vertebrate homologues, in accordance with quiescence-dependent $\mathrm{Rb}$ expression, p130/E2F4 interacts with similar histone modifying proteins as the LINC complex and has been found to be specific to quiescence (Litovchick et al., 2007). Protein analogues of the invertebrate LINC complex include vertebrate proteins F25965 and LOC91750. During quiescence, the $\mathrm{p} 130 / \mathrm{E} 2 \mathrm{~F} 4$ complex binds to more than 800 promoters and is found in association with E2F target gene promoters (Litovchick et al., 2007; Pilkinton et al., 2007; Andersen et al., 2007). It is the goal of this Chapter to elucidate how the downstream regulators of $\mathrm{G}_{0} / \mathrm{G}_{1}$ arrest ( $\mathrm{Rb}$ and $\mathrm{E} 2 \mathrm{~F}$ family members) are regulated and whether anoxia-induced cell cycle arrest in the turtle is specific to the $G_{1}$ or $G_{0}$ phases of the cell cycle. This chapter will explore the specific mechanisms of $\mathrm{G}_{0} / \mathrm{G}_{1}$ phase arrest of the cell cycle, exploring both the differential regulation and protein interactions that are markers of either type of cell cycle arrest. Specially, this study will determine the protein make-up of inhibitory transcriptional complexes built upon E2F family members as it is this transcriptional complex that directs cell cycle arrest to remain arrested in the $\mathrm{G}_{1}$ phase, or end reversible quiescence $\left(\mathrm{G}_{0}\right)$. 


\subsection{Materials and Methods}

\subsubsection{Animal Care and Treatment}

All animal experiments were conducted as described in Chapter 2.

\subsubsection{Total protein and nuclear isolation}

All protein isolations were conducted as described in Chapter 2.

\subsubsection{Immunoblotting}

Preparation of tissue extracts and western blotting were performed as described in Chapter 2 with minor modification. For western blotting, aliquots containing 30-40 $\mu \mathrm{g}$ protein from control and stress conditions were loaded into lanes of polyacrylamide gels (8-12\% gels depending on the MW of the protein being analyzed). Gels were run in a Mini Protean III apparatus (BioRad) at $180 \mathrm{~V}$ for $45 \mathrm{~min}$ at RT. The transfer was carried out at $160 \mathrm{~mA}$ constant amperage for $1.5 \mathrm{~h}$. The membranes were blocked using $1 \mathrm{mg} / \mathrm{ml}$ PVA (70-100 kDa) in TBST for 30-45 sec. After blocking, the membranes were probed with antigen-specific antibodies (see Appendix C). Experimental conditions that are specific to each antibody are listed in Appendix D. Immunoblots were developed using enhanced chemiluminescence reagents. 


\subsubsection{Electrophoretic mobility shift assay}

Nuclear extracts were prepared as in Chapter $\mathbf{2}$ with minor modification. Following protein normalization, to keep proteins in the native state, prepared nuclear extracts did not undergo the addition of 2X SDS buffer or boiling. Electrophoretic mobility shift assays were performed by using the LightShift ${ }^{\circledR}$ Chemiluminescent EMSA Kit (Pierce, Rockford, IL) with the following double-stranded 5' biotin-labeled oligonucleotide probes: 5'-Biotin-ATTTAAG the complementary sequence was 5'-TTGAGAAAGGGCGCGAAACTTAAAT-3'. The E2F DNA binding sequence is bold and underlined. Both oligonucleotides were purchased from Sigma Genosys, diluted to $500 \mu \mathrm{M}$ using sterile $\mathrm{H}_{2} \mathrm{O}$ and subsequently mixed 1:1 (v/v) for a total volume of $20 \mu \mathrm{l}$. Probes were then placed in a thermocycler for $10 \mathrm{~min}$ at $95^{\circ} \mathrm{C}$ and the temperature of the block was slowly cooled to RT. For each binding reaction $(10 \mu \mathrm{l})$, a total of $10 \mathrm{ng}$ biotin-labeled probe was combined with $5 \mu \mathrm{g}$ native protein nuclear extract (2X SDS buffer not added and samples not boiled), $1 \mu \mathrm{g}$ poly $(\mathrm{dI}-\mathrm{dC})$, and $1 \mathrm{X}$ Binding buffer (10 mM Tris, $\mathrm{pH}$ 7.8, $50 \mathrm{mM} \mathrm{NaCl,} 1 \mathrm{mM}$ EDTA, $5 \%$ glycerol). A commercially available positive control kit containing biotin-labeled probe for E2F and HeLa cell nuclear lysate was utilized to determine the success of cross reactivity (Panomics; Cat\#AY1010P). 'No protein' and 'no probe' controls were created by replacing the controlled component with the same volume of $\mathrm{ddH}_{2} \mathrm{O}$. To validate ability of the custom designed E2F DNA probe, competition assays were utilized with

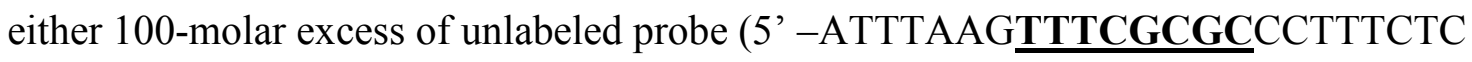
AA-3') or 100-molar excess of mutated ( $\left.{ }^{\sim}\right)$ unlabeled probe (5'-ATTTAAG $\underline{\text { TTGCGA }}$ GGCCTTTCTCAA-3'). 
A $6.0 \%$ non-denaturing polyacrylamide gel was prepared to separate out the complex. The gel was made up of $0.5 \mathrm{~mL}$ of chilled 10X TBE (0.89 M Tris Base, $0.89 \mathrm{M}$ boric acid, $4 \%$ of $0.5 \mathrm{M}$ EDTA, $\mathrm{pH} 8.0$ ), $2 \mathrm{~mL}$ of $30 \%$ Bis-acrylamide, $312 \mu 1$ of $80 \%$ glycerol, $7 \mathrm{~mL}$ of deionized, sterile water, $150 \mu 1$ of $10 \%$ APS and $10 \mu 1$ of TEMED. The gel was allowed to polymerize and then run with $0.5 \mathrm{X}$ TBE at $120 \mathrm{~V}$ for $10 \mathrm{~min}$ in the electrophoresis apparatus at $4{ }^{\circ} \mathrm{C}$. The prepared samples were mixed with $2 \mu 1$ of $6 \mathrm{X}$ DNA loading dye and loaded into the gel. The gel was run at $120 \mathrm{~V}$ for $40 \mathrm{~min}$ at $4{ }^{\circ} \mathrm{C}$. The complex was then transferred onto a Biodyne B nylon membrane (Pall Corporation; Cat\#60120) in a Mini Protean transfer apparatus (BioRad) with 0.5X TBE buffer at a constant $300 \mathrm{~mA}$ for $30 \mathrm{~min}$ at $4{ }^{\circ} \mathrm{C}$.

Following the transfer, the membrane was placed between two dry sheets of filter paper and placed in a dry oven (at $80^{\circ} \mathrm{C}$ for $1.5 \mathrm{~h}$ ) to allow the oligonucleotides to fix on the membrane. The membranes were transferred to a clean membrane box and incubated for $15 \mathrm{~min}$ in $5 \mathrm{~mL}$ of $1 \mathrm{X}$ blocking buffer (Panomics; Cat\#CS7535) at RT. Following this step, $1 \mathrm{~mL}$ of the $1 \mathrm{X}$ blocking buffer was removed and combined with $5 \mu 1$ of streptavidin-HRP (BioShop; Cat\#APA012P) and then added back to the membrane box. The membrane was allowed to rock for an additional $15 \mathrm{~min}$ at room temperature. The blocking solution was removed and the membrane was washed for 30 min with $1 \mathrm{X}$ washing buffer (Panomics; Cat\#CS7537).

The membrane was developed using enhanced chemiluminescence reagents and imaged using a Chemi-Genius BioImaging system (Syngene, Frederick, MD). Bands were visualized to qualitatively confirm the presence/absence of DNA binding by E2F. 


\subsubsection{Enzyme-linked immunosorbent assay}

Nuclear extracts were prepared as above. Aliquots containing equal amounts of protein from each sample (10-16 $\mu \mathrm{g} /$ well, depending on tissue) were then used to assess the amount of binding by E2F1 and E2F4 to their response element. This was done using an ELISA-type assay that uses 96-well microplates that were previously coated with an oligonucleotide corresponding to the DNA binding element that is recognized by E2F family of transcription factors.

The sequence of the biotin-conjugated probe was 5'-Biotin-ATTTAAG $\underline{\text { TTCG }}$ CGCCCTTTCTCAA-3', whereas the complementary sequence was 5'-TTGAGAAAG GGCGCGAAACTTAAAT-3'. A 40 pmol aliquot of double-stranded, biotinylated probe in $50 \mu \mathrm{L}$ of phosphate buffered saline (PBS; $137 \mathrm{mM} \mathrm{NaCl}, 2.7 \mathrm{mM} \mathrm{KCl}, 10 \mathrm{mM}$ $\mathrm{Na}_{2} \mathrm{HPO}_{4}, 2 \mathrm{mM} \mathrm{KH}{ }_{2} \mathrm{PO}_{4}, \mathrm{pH}$ 7.4) was then added to each well of a streptavidin-coated microplate (VWR; Cat\# 15520). The plate was then incubated at RT for $1 \mathrm{~h}$ and washed twice briefly with $1 \mathrm{X}$ wash buffer $(0.1 \%$ Tween-20 in PBS) and once with PBS. Aliquots of $10-16 \mu \mathrm{g}$ protein were combined with $50 \mu \mathrm{L}$ of $1 \mathrm{X}$ protein binding buffer $(10 \mathrm{mM}$ Hepes, pH 7.9, $50 \mathrm{mM} \mathrm{KCl,} 0.5 \mathrm{mM}$ EDTA, 3 mM MgCl $2,10 \%$ glycerol, $0.5 \mathrm{mg} / \mathrm{mL}$ BSA, 0.05\% NP-40, 1-2 $\mu$ g salmon sperm DNA (BioShop; Cat\# DNA999), 0.5 mM DTT, and $\mathrm{NaCl}$ (a variable amount was used based on the volume of nuclear extract added, final concentration was always $40 \mathrm{mM})$ ) and then added to the wells. Equal amounts of protein for all experimental conditions were used in each assay, and all experimental samples were always run together. Only binding buffer, without protein samples, was added to the negative control wells. The plate was incubated at room temperature with mild agitation for 75 min followed by four brief washes with $1 \mathrm{X}$ wash 
buffer. A $60 \mu \mathrm{L}$ aliquot of the E2F1 or E2F4 antibody (the same antibodies used for western blotting, see Appendix C), diluted 1:1000 v:v in PBS was added to the wells and the plate was incubated for $1 \mathrm{~h}$ at RT. The plate was washed as above and then $60 \mu \mathrm{L}$ of anti-rabbit IgG-HRP (BioShop; Cat\# APA007P) diluted 1:2000 v:v in PBS was added and the plate was incubated for $1 \mathrm{~h}$ at RT. Following this, the plate was washed as above and $60 \mu \mathrm{L}$ of TMB (tetramethylbenzidine) was added (Bioshop; Cat\#TMB333.100). Once color had developed, the reaction was stopped by the addition of $1 \mathrm{M} \mathrm{HCl}$ and optical density was read at $450 \mathrm{~nm}$ with a reference wavelength of $655 \mathrm{~nm}$ using a Multiskan Spectrum (Thermo Labsystems).

\subsubsection{DNA-bound protein complex enzyme-linked immunosorbent assay}

Nuclear extracts for DNA-binding assay were prepared as previously described for both EMSA and ELISA protocols. Aliquots containing equal amounts of protein from each sample (16-24 $\mu \mathrm{g} /$ well) were then used to assess the amount of binding by E2F1 and E2F4 to the E2F DNA binding sequence. The sequence of the biotin-conjugated probe was the same as that used for both E2F EMSA and ELISA protocols. A 40 pmol aliquot of double-stranded, biotinylated probe in $50 \mu \mathrm{L}$ of phosphate buffered saline (PBS; 137 $\mathrm{mM} \mathrm{NaCl}, 2.7 \mathrm{mM} \mathrm{KCl}, 10 \mathrm{mM} \mathrm{Na}_{2} \mathrm{HPO}_{4}, 2 \mathrm{mM} \mathrm{KH}_{2} \mathrm{PO}_{4}, \mathrm{pH}$ 7.4) was then added to each well of a streptavidin-coated microplate. The plate was then incubated at RT for $1 \mathrm{~h}$ and washed twice with wash buffer (0.1\% Tween-20 in PBS) and once with PBS.

Aliquots of 16-24 $\mu \mathrm{g}$ protein were combined with $50 \mu \mathrm{L}$ of $1 \mathrm{X}$ protein binding buffer (10 mM Hepes, pH 7.9, 50 mM KCl, 0.5 mM EDTA, 3 mM $\mathrm{MgCl}_{2}, 10 \%$ glycerol, 0.5 $\mathrm{mg} / \mathrm{mL}$ BSA, $0.05 \%$ NP-40, 1-2 $\mu \mathrm{g}$ salmon sperm DNA, $0.5 \mathrm{mM}$ DTT, and $80 \mathrm{mM}$ 
$\mathrm{NaCl}$ ) and then added to the wells. Equal amounts of protein for all experimental conditions were used in each assay, and all experimental samples were always run together. Only binding buffer, without protein samples, was added to the negative control wells. The plate was incubated at room temperature with mild agitation for 60-90 min followed by four washes with $1 \mathrm{X}$ wash buffer. A $60 \mu \mathrm{L}$ aliquot of the appropriate antibody, diluted in PBS, was added to the wells and the plate was incubated for 1-1.5 $\mathrm{h}$ at RT. The plate was washed as above and then $60 \mu \mathrm{L}$ of appropriate IgG-HRP secondary antibody diluted 1:2000 v:v in PBS was added and the plate was incubated for $1 \mathrm{~h}$ at RT. Following this, the plate was washed as above and $60 \mu \mathrm{L}$ of TMB (tetramethylbenzidine) was added. Once colour had developed, the reaction was stopped by the addition of $1 \mathrm{M}$ $\mathrm{HCl}$ and optical density was read at $450 \mathrm{~nm}$ with a reference wavelength of $655 \mathrm{~nm}$ using a Multiskan Spectrum. Since this was a newly optimized technique developed for this thesis, buffer optimization figures can be seen in Appendix J.

For both E2F1 and E2F4, $16 \mu \mathrm{g}$ of protein was combined with $50 \mu \mathrm{L}$ of $1 \mathrm{X}$ protein binding buffer. The plate was incubated at RT with mild agitation for $60 \mathrm{~min}$ and then washed 4 times with $1 \mathrm{X}$ wash buffer. Aliquots of $60 \mu \mathrm{L}$ of the respective antibody, diluted 1:1000 in PBS, were then added to the wells and the plate was incubated for $1 \mathrm{~h}$. The plate was washed as above and then $60 \mu \mathrm{L}$ of appropriate anti-rabbit IgG-HRP diluted 1:2000 in PBS was added and the plate was incubated for $1 \mathrm{~h}$. Data acquisition and analysis was the same as described earlier in section 4.2.5.

For $\mathrm{Rb}$ and $\mathrm{HDAC} 4,16 \mu \mathrm{g}$ of protein was combined with $50 \mu \mathrm{L}$ of $1 \mathrm{X}$ protein binding buffer. The plate was incubated at RT with mild agitation for 90 min and then washed four times with $1 \mathrm{X}$ wash buffer. Aliquots of $60 \mu \mathrm{L}$ of the respective antibody, 
diluted 1:1000 in PBS, were then added to the wells and the plate was incubated for $1.5 \mathrm{~h}$ at RT. The plate was washed as above and then $60 \mu \mathrm{L}$ of 1:500 anti-mouse IgG-HRP (for $\mathrm{Rb}$ ) or 1:1000 anti-rabbit IgG-HRP (for HDAC4) in PBS was added and the plate was incubated for $1 \mathrm{~h}$ at RT. The remainder of the procedure was as described earlier.

For $\mathrm{p} 130,24 \mu \mathrm{g}$ of protein was combined with $50 \mu \mathrm{L}$ of $1 \mathrm{X}$ protein binding buffer. The plate was incubated at RT with mild agitation for $90 \mathrm{~min}$ and then washed four times with $1 \mathrm{X}$ wash buffer. Aliquots of $60 \mu \mathrm{L}$ of the p130 antibody, diluted 1:500 $\mathrm{v}: \mathrm{v}$ in PBS, were then added to the wells and the plate was incubated for $1.5 \mathrm{~h}$ at RT. The plate was washed as above and then $60 \mu \mathrm{L}$ of appropriate anti-goat IgG-HRP diluted 1:4000 v:v in PBS was added and the plate was incubated for $1 \mathrm{~h}$ at RT. The remainder of the procedure was as described previously.

For Suv39H1, $16 \mu \mathrm{g}$ of protein was combined with $50 \mu \mathrm{L}$ of $1 \mathrm{X}$ protein binding buffer. The plate was incubated at RT with mild agitation for $90 \mathrm{~min}$ and then washed four times with $1 \mathrm{X}$ wash buffer. Aliquots of $60 \mu \mathrm{L}$ of the Suv39H1 antibody, diluted $1: 1000 \mathrm{v}: \mathrm{v}$ in PBS, were then added to the wells and the plate was incubated for $1.5 \mathrm{~h}$ at RT. The plate was washed as above and then $60 \mu \mathrm{L}$ of appropriate anti-goat IgG-HRP diluted 1:4000 v:v in PBS was added and the plate was incubated for $1 \mathrm{~h}$ at RT. The remainder of the procedure was as described previously.

\subsubsection{Total RNA isolation}

RNA extraction was performed as described in Chapter 2. 


\subsection{8 cDNA synthesis}

cDNA synthesis was performed as described in Chapter 2.

\subsubsection{Polymerase chain reaction}

PCR amplification was performed as described in Chapter 2. Polymerase Chain Reaction (PCR) was used to amplify the sequences under study from the cDNA samples. Each PCR reaction consisted of $13.25 \mu \mathrm{L}$ of sterile water, $5 \mu \mathrm{L}$ of diluted cDNA, $1.25 \mu \mathrm{L}$ of $1.5 \mu \mathrm{M}$ primer mixture (see Appendix F), $2.5 \mu \mathrm{L}$ of 10X PCR buffer (Invitrogen; Cat\# N8080006), $1.5 \mu \mathrm{L}$ of $50 \mathrm{mM} \mathrm{MgCl} 2,0.5 \mu \mathrm{L}$ of $25 \mathrm{mM}$ dNTPs and $1 \mu \mathrm{L}$ of Taq polymerase, for a total volume of $25 \mu \mathrm{L}$. All PCR amplification cycles were as follows; an initial denaturation at $94{ }^{\circ} \mathrm{C}$ for $7 \mathrm{~min}$, followed by an experimentally determined number of cycles of $94{ }^{\circ} \mathrm{C}$ for 1 min, primer annealing $50-60{ }^{\circ} \mathrm{C}$ (experimentally determined; see Appendix G) for $1 \mathrm{~min}$, and elongation at $72{ }^{\circ} \mathrm{C}$ for $1 \mathrm{~min}$. The final elongation was at $72{ }^{\circ} \mathrm{C}$ for $10 \mathrm{~min}$.

\subsubsection{Sequencing}

All PCR products were sequenced by BioBasics (Markham, ON) as described in

\section{Chapter 2.}

\subsubsection{Statistics}

Data analysis was performed as described in Chapter 2. 


\subsection{Results}

\subsubsection{Regulation of E2F expression and intra-cellular localization}

Both E2F1 and E2F4 antibodies cross-reacted with a protein band of the molecular weight of either 72 or $50 \mathrm{kDa}$, respectively (Figure 4.2). Changes in the relative expression of E2F1 (active) and E2F4 (repressive) protein in response to anoxia were assessed using immunoblotting with analysis of their expression in both whole-cell (Figure 4.3) and nuclear protein lysates (Figure 4.4). In liver tissue, the total protein expression of E2F1 decreased to $61 \pm 10$ after $5 \mathrm{~h}$ and to $75 \pm 2 \%$ after $20 \mathrm{~h}$ anoxia of control values $(P<0.05)$. Nuclear expression of E2F1 in liver displayed a decrease in protein expression only after $20 \mathrm{~h}$ anoxia, decreasing to $24 \pm 7 \%$ of control values $(P<$ $0.05)$. In kidney, the total protein expression of E2F1 decreased to $73 \pm 1 \%$ of control values after $20 \mathrm{~h}$ anoxia $(P<0.05)$ and nuclear expression of E2F1 followed the same pattern, decreasing to $74 \pm 6 \%$ of control values after $20 \mathrm{~h}$ anoxia $(P<0.05)$. In white skeletal muscle, the total protein expression of E2F1 significantly changed only in response to $20 \mathrm{~h}$ anoxia, decreasing to $50 \pm 12 \%$ of control values $(P<0.05)$. The nuclear expression of E2F1 from white skeletal muscle displayed a decrease in protein expression to $44 \pm 6$ after $5 \mathrm{~h}$ and to $60 \pm 8 \%$ after $20 \mathrm{~h}$ anoxia of control values $(P<0.05)$.

The protein expression of E2F4 displayed a different pattern of response to anoxia when compared to E2F1. In liver tissue, the total protein expression of E2F4 significantly increased by $1.26 \pm 0.05$-fold after $5 \mathrm{~h}$ and by $1.35 \pm 0.04$-fold after $20 \mathrm{~h}$ anoxia as compared to control values $(P<0.05)$. Nuclear expression of E2F4 in liver extracts displayed a significant increase in protein expression only after $20 \mathrm{~h}$ anoxia, increasing by $1.44 \pm 0.03$-fold compared to control values $(P<0.05)$. Similar to the pattern for liver, 
the total protein expression of E2F4 from kidney tissue increased by $1.57 \pm 0.10$-fold after $20 \mathrm{~h}$ anoxia as compared to control values $(P<0.05)$ and also rose significantly in nuclear extracts of kidney by $1.76 \pm 0.11$-fold compared to control values after $20 \mathrm{~h}$ anoxia $(P<0.05)$. In white skeletal muscle, the total protein expression of E2F4 increased by $2.09 \pm 0.26$-fold after $20 \mathrm{~h}$ anoxia and by $2.97 \pm 0.03$-fold in nuclear extracts, when compared to control values $(P<0.05)$.

\subsubsection{Regulation of Retinoblastoma expression and relative phosphorylation}

Changes in the relative expression of $\mathrm{Rb}$ protein and the associated phosphorylation and acetylation states of $\mathrm{Rb}$ in response to anoxia were assessed using immunoblotting with whole-cell protein lysates (Figure 4.5). All Rb antibodies used in this study cross-reacted with a protein band of $\sim 110 \mathrm{kDa}$ (Figure 4.2). No significant changes in total $\mathrm{Rb}$ expression were detected in liver or white skeletal muscle tissues in response to either 5 or $20 \mathrm{~h}$ anoxia. However, kidney tissue showed a significant increase in total $\mathrm{Rb}$ expression of $1.54 \pm 0.07$ and $2.08 \pm 0.21$-fold after 5 and $20 \mathrm{~h}$ anoxia, respectively, when compared to control values $(P<0.05)$. Levels of $\mathrm{p}-\mathrm{Rb}$ (Ser780) decreased in response to anoxia in both kidney and white skeletal muscle tissues. In kidney, the relative expression of $\mathrm{p}-\mathrm{Rb}$ (Ser780) decreased to $60 \pm 6$ and $71 \pm 9 \%$ of control values in response to 5 and $20 \mathrm{~h}$ anoxia, respectively $(P<0.05)$. In white skeletal muscle, the relative expression of $\mathrm{p}-\mathrm{Rb}$ (Ser780) decreased to $70 \pm 3 \%$ of control values in response to $20 \mathrm{~h}$ anoxia $(P<0.05)$. Conversely, the relative levels of $\mathrm{p}-\mathrm{Rb}$ (Ser780) in liver significantly increased by $2.20 \pm 0.07$-fold in response to $5 \mathrm{~h}$ anoxia but then significantly decreased back to control values after $20 \mathrm{~h}$ anoxia $(P<0.05)$. The critical 
phosphorylation site for inactivation of Rb is Ser608. Phosphorylation of this residue induces a conformation change of the protein and release from its inhibitory action on E2F proteins. The relative levels of $\mathrm{p}-\mathrm{Rb}$ (Ser608) decreased in both liver and kidney tissues in response to anoxia but were unchanged in muscle. In liver tissue, the relative

expression of $\mathrm{p}-\mathrm{Rb}$ (Ser608) decreased to $58 \pm 1$ and $74 \pm 4 \%$ of control values in response to 5 or $20 \mathrm{~h}$ anoxia, respectively $(P<0.05)$. Similar to liver tissue, the relative expression of $\mathrm{p}-\mathrm{Rb}$ (Ser608) in kidney tissue decreased to $66 \pm 15$ and $65 \pm 11 \%$ of control values in response to 5 or $20 \mathrm{~h}$ anoxia, respectively $(P<0.05)$. Apart from the mainly inhibitory roles phosphorylation on $\mathrm{Rb}$ function, acetylation of $\mathrm{Rb}$ protein has been shown to increase function through the inhibition of phosphorylation. The relative levels of acetyl$\mathrm{Rb}$ (Lys873) increased in both liver and kidney tissues in response to anoxia. In liver tissue, the relative levels of acetyl- $\mathrm{Rb}$ (Lys873) increased by $2.45 \pm 0.38$ and $2.92 \pm 0.26$ fold compared to control values in response to 5 or $20 \mathrm{~h}$ anoxia, respectively $(P<0.05)$. Similar to liver, the relative expression of acetyl-Rb (Lys873) in kidney tissue increased by $1.30 \pm 0.08$ and $1.55 \pm 0.02$-fold compared to control values in response to either 5 or 20 $\mathrm{h}$ anoxia, respectively $(P<0.05)$. Acetyl-Rb (Lys873) levels in white skeletal muscle were unaffected by anoxia.

\subsubsection{Regulation of Retinoblastoma intra-cellular localization}

The nuclear expression of $\mathrm{Rb}$ targets was also assessed using immunoblotting (Figure 4.6). In liver tissue, the nuclear expression of Rb protein did not significantly increase from control values in response to either 5 or $20 \mathrm{~h}$ anoxia; however, after $20 \mathrm{~h}$ anoxia, the nuclear protein expression significantly increased from $5 \mathrm{~h}$ levels $(P<0.05)$. 
The nuclear expression of total $\mathrm{Rb}$ protein increased in response to anoxia in both kidney and white skeletal muscle tissues. The nuclear expression of Rb protein in kidney tissue increased $2.65 \pm 0.16$ and $2.83 \pm 0.22$-fold compared to control values in response to 5 and $20 \mathrm{~h}$ anoxia, respectively $(P<0.05)$ whereas in white skeletal muscle, the nuclear $\mathrm{Rb}$ protein increased by $1.54 \pm 0.18$-fold compared to control values after $20 \mathrm{~h}$ of anoxia $(P<$ 0.05). Levels of $\mathrm{p}-\mathrm{Rb}$ (Ser780) did not significantly change in response to anoxia in liver. In kidney tissue, the relative expression of $\mathrm{p}-\mathrm{Rb}$ (Ser780) significantly decreased to $50 \pm 10 \%$ of control values after $20 \mathrm{~h}$ anoxia $(P<0.05)$ whereas in white skeletal muscle $\mathrm{p}-\mathrm{Rb}$ (Ser780) decreased to $43 \pm 3 \%$ of controls $(P<0.05)$. In liver and skeletal muscle, the relative nuclear levels of $\mathrm{p}-\mathrm{Rb}$ (Ser608) did not significantly change in response to either 5 or $20 \mathrm{~h}$ anoxia. However, the relative nuclear levels of $\mathrm{p}-\mathrm{Rb}$ (Ser608) in kidney tissue initially increased by $1.42 \pm 0.04$-fold in response to $5 \mathrm{~h}$ anoxia, but then decreased to $52 \pm 11 \%$ of control values in response to $20 \mathrm{~h}$ anoxia $(P<0.05)$.

The relative nuclear levels of acetyl-Rb (Lys873) increased in all tissues studied in response to either 5 or $20 \mathrm{~h}$ anoxia. In liver tissue, the relative nuclear levels of acetyl$\mathrm{Rb}$ (Lys 873 ) increased by $1.31 \pm 0.05$ and $1.40 \pm 0.06$-fold compared to control values in response to 5 and $20 \mathrm{~h}$ anoxia, respectively $(P<0.05)$. In kidney acetyl-Rb (Lys873) content increased by $2.63 \pm 0.25$-fold in response to $20 \mathrm{~h}$ anoxia $(P<0.05)$ and in white skeletal muscle levels increased by $1.61 \pm 0.18$ and $1.59 \pm 0.28$-fold compared to control values after 5 and $20 \mathrm{~h}$ anoxia, respectively $(P<0.05)$. 


\subsubsection{Regulation of p130 expression and intra-cellular localization}

Changes in the relative expression of p130 protein in response to anoxia were assessed using immunoblotting in both whole-cell (Figure 4.7) and nuclear protein lysates (Figure 4.8). The p130 antibodies used in this study cross-reacted with a protein band of $\sim 130 \mathrm{kDa}$ (Figure 4.2). In liver tissue, the total protein expression of p130 significantly decreased to $49 \pm 6 \%$ of control values after $5 \mathrm{~h}$ of anoxia but rose again to $1.32 \pm 0.06$-fold higher than control values after $20 \mathrm{~h}$ anoxia $(P<0.05)$. The same pattern was seen for the nuclear expression of p130 from liver which significantly decreased to $63 \pm 5 \%$ of control values after $5 \mathrm{~h}$ of anoxia and then significantly increased to $1.26 \pm 0.08$-fold over control values after $20 \mathrm{~h}$ anoxia $(P<0.05)$. The total protein expression of p130 from kidney tissue increased by $1.63 \pm 0.07$ after $5 \mathrm{~h}$ and by $2.8 \pm 0.10$-fold after $20 \mathrm{~h}$ anoxia when compared to control values $(P<0.05)$. Similar to total protein expression, the nuclear expression of p130 from kidney displayed an increase of $1.85 \pm 0.11$ after $5 \mathrm{~h}$ and of $2.43 \pm 0.17$-fold after $20 \mathrm{~h}$ anoxia as compared to control values $(P<0.05)$. No significant changes in either total or nuclear expression of p130 were detected in white skeletal muscle in response to either $5 \mathrm{~h}$ or $20 \mathrm{~h}$ anoxia.

\subsubsection{Regulation of E2F-associated epigenetic modifiers and their intracellular localization}

Changes in the relative expression of HDAC4 and Suv39H1 protein in response to anoxia were assessed using immunoblotting to examine both whole-cell (Figure 4.9) and nuclear protein (Figure 4.10) lysates. All antibodies cross-reacted with a protein band of either $\sim 140 \mathrm{kDa}$ or $\sim 48 \mathrm{kDa}$, the expected size of HDAC4 and Suv39H1, respectively 
(Figure 4.2). In liver tissue, the total protein expression of Suv39H1 significantly increased by $1.34 \pm 0.08$-fold after $20 \mathrm{~h}$ anoxia when compared to control values $(P<$ 0.05). However, nuclear expression of Suv39H1 in liver did not significantly change in response to anoxia relative to the control. Total protein expression of Suv39H1 in kidney increased by $1.15 \pm 0.06$ after $5 \mathrm{~h}$ and further increased to $1.36 \pm 0.04$-fold after $20 \mathrm{~h}$ anoxia compared to controls $(P<0.05)$ and the nuclear expression of Suv39H1 also displayed small, but significant, increases in protein expression of $1.12 \pm 0.06$ after $5 \mathrm{~h}$ and of $1.15 \pm 0.01$ after $20 \mathrm{~h}$ anoxia as compared to control values $(P<0.05)$. In white skeletal muscle, the total protein expression of Suv39H1 increased by $1.78 \pm 0.09$-fold after $20 \mathrm{~h}$ anoxia compared to controls $(P<0.05)$ whereas nuclear expression of the protein increased by $1.35 \pm 0.04$ after $5 \mathrm{~h}$ anoxia and $1.52 \pm 0.03$-fold after $20 \mathrm{~h}$ anoxia when compared to control values $(P<0.05)$.

In liver tissue, the total protein expression of HDAC4 increased by $2.52 \pm 0.14$ after $20 \mathrm{~h}$ anoxia as compared to controls $(P<0.05)$. Nuclear expression of HDAC4 in liver also increased by $1.44 \pm 0.04$ after $5 \mathrm{~h}$ and of $2.34 \pm 0.11$ after $20 \mathrm{~h}$ anoxia when compared to control values $(P<0.05)$. In kidney tissue, total HDAC4 protein expression increased by $1.50 \pm 0.03$-fold after $5 \mathrm{~h}$ and by $1.35 \pm 0.04$-fold after $20 \mathrm{~h}$ anoxia, as compared to controls $(P<0.05)$. Nuclear expression of HDAC4 from kidney showed comparable increases of $1.61 \pm 0.09$-fold after $5 \mathrm{~h}$ anoxia and $1.39 \pm 0.11$-fold after $20 \mathrm{~h}$ anoxia $(P<0.05)$. In white skeletal muscle, total protein expression of HDAC4 increased by $1.77 \pm 0.02$-fold over controls after $20 \mathrm{~h}$ anoxia $(P<0.05)$ and nuclear HDAC4 expression also increased by $1.58 \pm 0.01$-fold after $20 \mathrm{~h}(P<0.05)$. 


\subsubsection{Regulation of E2F DNA-binding activity during anoxia}

The transcription factor E2F regulates many genes involved in DNA replication, differentiation and pro-apoptosis. In order to determine whether E2F could bind the DNA probes utilized in the ELISA experiments, the relative qualitative level of E2F binding to DNA was visualized in extracts from control and anoxic liver of T. scripta elegans. The interaction between $\mathrm{E} 2 \mathrm{~F}$ and the promoter sequence to which it binds was investigated using an electrophoretic mobility shift assay with biotin-labeled oligonucleotides containing the E2F binding site (5'-TTTCGCGC-3'). Figure 4.11 shows that the biotinlabeled probes bound to the E2F protein in extracts from control, $5 \mathrm{~h}$ anoxic and $20 \mathrm{~h}$ anoxic liver; results also show a commercial E2F probe (positive control) bound to E2F protein from the supplied HeLa cell lysate. Negative controls included protein without biotin-probes (no probe) as well as biotin-probes without protein (no protein). The amount of E2F binding between stresses was not quantified since several E2F family members (E2F1-7), all of similar molecular weight, bind the same DNA sequence.

The DNA-binding activity of E2F1 and E2F4 in liver, kidney and white skeletal muscle from normoxic, $5 \mathrm{~h}$ and $20 \mathrm{~h}$ anoxic turtles was assessed using a transcription factor ELISA. To test the validity of the custom-designed DNA probe containing the E2F-DNA binding sequence, we carried out probe competition studies (Figure 4.12). Competition between the biotin-labeled (capture) probe and 100-molar excess of unlabeled probe (wild-type) resulted in a significant reduction to $12 \pm 2 \%$ of the noncompetition control signal $(\mathrm{P}<0.05)$. A 3 bp mutation of the 8 bp E2F binding site within the wild-type competing probe (100-molar excess) significantly restored detection to $42 \pm 3 \%$ of control levels, a $3.42 \pm 0.26$-fold increase from the wild-type competition $(P<$ 
0.05). Combined with the EMSA results, these findings validate that the E2F-DNA binding probe works specifically to bind E2F transcription factors.

A significant decrease in E2F1 DNA binding activity was evident in response to 5 and $20 \mathrm{~h}$ of anoxia in both liver and kidney tissues but no significant changes were seen in muscle (Figure 4.13). The DNA binding activity of E2F1 decreased to $42 \pm 7 \%$ of control values after $20 \mathrm{~h}$ anoxia in liver tissue $(P<0.05)$. In kidney, relative DNA binding activity of E2F1 significantly decreased to $75 \pm 2$ and $61 \pm 2 \%$ of control values after 5 and $20 \mathrm{~h}$ anoxia, respectively $(P<0.05)$. The opposite DNA binding pattern was seen when E2F4 was examined. Binding increased by $1.71 \pm 0.11$ and $2.57 \pm 0.26$-fold in liver and by $1.47 \pm 0.14$ and $2.08 \pm 0.29$-fold in kidney tissues in response to 5 and $20 \mathrm{~h}$ anoxia, respectively, when compared to control values $(P<0.05)$. Again, no significant change was seen in muscle..

\subsubsection{Analysis of active DNA-binding E2F-based protein complexes during anoxia}

Formation of primary DNA-binding complexes in response to anoxia was assessed by using polyclonal antibodies to detect the presence of proteins known to bind to E2F transcription factors (Figure 4.14). These complex proteins include Rb and p130, both of which bind directly to E2F transcription factors, allowing the assessment of primary complex formation. In the liver, the E2F-binding ability of Rb increased by $2.48 \pm 0.21$-fold after $20 \mathrm{~h}$ of anoxia, when compared to control values $(P<0.05)$. However, binding of p130 did not show any significant differences between control and anoxic conditions. In kidney, the E2F-binding ability of Rb increased by $1.57 \pm 0.06$-fold in response to $5 \mathrm{~h}$ and by $1.66 \pm 0.14$-fold in response to $20 \mathrm{~h}$ of anoxia, as compared to 
controls $(P<0.05)$. The relative E2F-binding of p130 also increased by $1.69 \pm 0.25$-fold over control values in response to $20 \mathrm{~h}$ anoxia in kidney tissue $(P<0.05)$. Both $\mathrm{Rb}$ and p130 did not change significantly in E2F-binding ability between control and anoxic conditions in white skeletal muscle.

The formation of secondary DNA-binding complexes in response to anoxia was assessed by detecting proteins known to bind Retinoblastoma family members ( $\mathrm{Rb}$ and p130) only when bound to E2F. These secondary complex proteins include HDAC4 and Suv39H1, both of which bind directly to Retinoblastoma family members ( $\mathrm{Rb}$ or $\mathrm{p} 130)$, allowing the assessment secondary complex formation (Figure 4.14). In the liver, the secondary-binding activity of HDAC4 increased by $3.88 \pm 0.44$-fold in response to $5 \mathrm{~h}$ and by $2.90 \pm 0.55$-fold in response to $20 \mathrm{~h}$ of anoxia when compared to control values $(P<$

0.05). Liver Suv39H1 showed no significant changes in binding after $5 \mathrm{~h}$ anoxia, but after $20 \mathrm{~h}$ anoxia displayed an increase of $1.50 \pm 0.16$-fold increase, compared to control values $(P<0.05)$. In kidney, the secondary-binding activity of HDAC4 increased by $2.15 \pm 0.38$ fold in response to $20 \mathrm{~h}$ of anoxia as compared to controls $(P<0.05)$. Suv39H1 from kidney also showed a significant increase in binding after $20 \mathrm{~h}$ anoxia by $1.76 \pm 0.31$-fold compared to controls $(P<0.05)$. Both HDAC4 and Suv39H1 did not show any significant differences in secondary-binding ability between control and anoxic conditions in white skeletal muscle.

\subsubsection{Regulation of PCNA expression}

The relative expression of PCNA protein and mRNA transcripts were used to assess the transcriptional activity of the E2F family of transcription factors (Figure 4.15). 
Using RT-PCR and consensus primers, the partial sequence of pcna was obtained and the relative expression of pcna transcripts was assessed in response to anoxia in turtle tissues (Figure 4.15A). In liver and white skeletal muscle, the relative expression of pcna transcripts did not change in response to anoxia $(P<0.05)$. However, the relative transcript levels of pcna significantly decreased after $20 \mathrm{~h}$ anoxia to $47 \pm 4 \%$ of control values in kidney $(P<0.05)$. The sequence of the pcna amplicon and the translated protein sequence are presented in Figure 4.16A. This amplicon represented 31\% of the comparable human nucleotide sequence and displayed a homology of $84 \%$ with the human nucleotide sequence and 91\% homology with chicken (Figure 4.16B). Similarly, the translated amino acid sequence represented $48 \%$ of the comparable human sequence and a homology of $95 \%$ and $99 \%$ as compared with the human and chicken sequences (Figure 4.16C).

Antibodies specific to PCNA protein were used to assess protein expression (Figure 4.15B). In liver and white skeletal muscle tissues, the relative expression of PCNA protein did not change in response to anoxia $(P<0.05)$. However, the relative expression of PCNA protein significantly decreased after $20 \mathrm{~h}$ anoxia to $70 \pm 1 \%$ of control values in kidney $(P<0.05)$.

\subsubsection{Regulation of mRNA transcripts levels of proteins involved in $G_{1}$ arrest}

RT-PCR was used to determine if the relative expression of transcripts that are associated with $\mathrm{G}_{1}$ arrest proteins showed altered expression correlating with increased protein expression in response to anoxia. The $\mathrm{G}_{1}$ arrest associated genes examined were $e 2 f 4, r b$ and $p 130$ (Figure 4.17). In liver tissue, the transcript levels of $e 2 f 4$ increased to 
$2.03 \pm 0.08$-fold higher than the control value in response to $5 \mathrm{~h}$ anoxia and further increased to $3.65 \pm 0.31$-fold of control after $20 \mathrm{~h}$ of anoxia $(P<0.05)$. In kidney, $e 2 f 4$ transcript levels increased to $1.89 \pm 0.11$-fold over control values after $20 \mathrm{~h}$ anoxia $(P<$ $0.05)$. In white skeletal muscle, no changes in the relative transcript expression of $e 2 f 4$ in response to anoxia were found. In liver, the transcript levels of $r b$ increased to $2.39 \pm 0.15$ fold over control values in response to $20 \mathrm{~h}$ anoxia $(P<0.05)$ but were unchanged after 5 $\mathrm{h}$ anoxia. In kidney, the transcript levels of $r b$ increased to $2.10 \pm 0.21$-fold higher than the control values in response to $5 \mathrm{~h}$ anoxia and further increased to $2.84 \pm 0.41$-fold of control after $20 \mathrm{~h}$ of anoxia $(P<0.05)$. In white skeletal muscle tissue, no changes in the relative transcript levels of $r b$ occurred in response to anoxia. Showing a similar trend in expression to that of $r b$ transcripts, the transcript levels of $p 130$ from liver tissue increased to $1.86 \pm 0.11$-fold over control values in response to $20 \mathrm{~h}$ anoxia $(P<0.05)$. In kidney tissue, the transcript levels of $p 130$ increased to $1.94 \pm 0.15$-fold higher than the control value in response to $5 \mathrm{~h}$ anoxia and remained high at $1.84 \pm 0.06$-fold over control after $20 \mathrm{~h}$ of anoxia $(P<0.05)$. In white skeletal muscle, no changes in the relative transcript expression of $p 130$ in response to anoxia were found.

The amplified transcripts and associated translated protein sequences for $e 2 f 4, r b$ and p130 amplicons are presented in Figures 4.18A, 4.19A and 4.20A, respectively. These amplicons represented 7, 13 and 16\% of the comparable human sequence and displayed homologies of 71,70 and $74 \%$ for $e 2 f 4, r b$ and $p 130$ amplicons, respectively, as compared with the human nucleotide sequence (Figure 4.18B, 4.19B and 4.20B, respectively). Similarly, the translated amino acid sequence represented 10, 22 and $22 \%$ of the associated human sequence and displayed homologies of 72,71 and $76 \%$ for E2F4, 
$\mathrm{Rb}$ and p130, respectively (Figure 4.18C, 4.19C and 4.20C, respectively). Interestingly, homologies with the chicken protein sequence were substantially higher at 75, 80 and 100 $\%$, respectively. 


\subsection{Discussion}

A typical $\mathrm{G}_{1} / \mathrm{G}_{0}$ arrest includes a switch from E2F1 (active) to E2F4 (repressive) DNA binding, in addition to the recruitment of proteins and enzymes such as $\mathrm{Rb}$, Suv39H1 and HDACs (Luo et al., 1998). The type and duration of cell cycle arrest is dictated by the particular proteins found complexed with E2F. Active, proliferating cells do not maintain this complex and only display active E2F1 DNA binding. The E2F complex during a reversible, short-term arrest $\left(\mathrm{G}_{1}\right.$ arrest $)$ has been shown to include: E2F4, Rb, HDAC4 and Suv39H1 (Habour and Dean, 2000). Complete exit from the cell cycle (reversible Quiescence or $\mathrm{G}_{0}$ ) is characterized by the same complex components exceptthat $\mathrm{Rb}$ is replaced with p130 and several auxiliary histone binding proteins are also added (Vairo et al., 1995). Studies examining interactions of Rb and p130 with E2F during cell growth and differentiation have shown that there are differences between these two proteins and their interactions with E2F. In general, Rb can bind E2F1 and E2F4, whereas p130 can only bind to E2F4 (Hijmans et al., 1995; Nevin, 1998). P130/E2F4 is specific to, and the most abundant complex in, quiescent cells and during differentiation in skeletal muscle. For example, $\mathrm{Rb} / \mathrm{E} 2 \mathrm{~F}$ complexes are replaced by p130/E2F4 complexes during quiescence, which is required to maintain inhibition of DNA synthesis. It is unclear why such as switch to p130/E2F4 is functionally important in quiescent cells, but it is an evolutionarily conserved mechanism.

\subsubsection{Specific mechanisms of cell cycle arrest in liver tissue}

Analysis of protein and mRNA expression, in conjunction with the analysis of the proteins actively bound to DNA-binding E2F proteins, provided insight into the 
regulatory mechanisms of anoxia induced cell cycle arrest. To test the DNA binding capacity of E2F during anoxia, custom DNA probes were designed, tested (Figure 4.11) and validated (Figure 4.12) specifically for use with T. scripta elegans. The ability of turtle specific E2F protein to bind this DNA probe (containing the E2F DNA binding sequence) was qualitatively visualized through the use of an EMSA and the banding patterns were similar between the turtle and mammalian controls (Figure 4.11). For additional validation of probe specificity, a competition assay displayed both reduced DNA binding with the addition of 100-molar excess of unlabeled probe, and partial restoration of detectable binding when the competitive probe was mutated within the binding sequence (Figure 4.12).

Upon entry into the anoxic state, a switch from E2F1 (active) to E2F4 (repressive) protein after $20 \mathrm{~h}$ of anoxia was evident in turtle liver. After $20 \mathrm{~h}$ of anoxia it was found that both the whole cell and nuclear levels of E2F1 significantly decreased (Figures 4.3 and 4.4). Interestingly, the decrease in E2F1 protein was correlated with an increase in repressive E2F4 protein in the same cellular fractions. Additionally, e2f4 transcript levels also increased after $5 \mathrm{~h}$ of anoxia. These findings suggest a mechanism where E2F4 may replace E2F1 on its DNA binding site during extended periods of anoxia $(20 \mathrm{~h})$ in liver. Indeed, the DNA binding activity of E2F1 was shown to significantly decrease after $20 \mathrm{~h}$ of anoxia whereas E2F4 binding increased at the same time-point. Although these findings, along with those presented in Chapter 2, further suggest $\mathrm{G}_{1} / \mathrm{G}_{0}$ arrest during periods of anoxia, they fail to distinguish between $\mathrm{G}_{1}$ arrest and quiescence $\left(\mathrm{G}_{0}\right)$. To distinguish between these two types of cell cycle arrest, the anoxic regulation of 
Retinoblastoma proteins ( $\mathrm{Rb}$ and $\mathrm{p} 130)$ and the arrest-specific DNA binding protein complexes were analyzed.

Overall analysis of Retinoblastoma proteins and arrest-specific DNA binding complexes identified a possible cell cycle arrest mechanism for anoxic turtle liver tissue. Results suggested that $\mathrm{Rb}$ is active after $20 \mathrm{~h}$ of anoxia. The expression of nuclear $\mathrm{Rb}$ protein increased after $20 \mathrm{~h}$ of anoxia, corresponding with increases in $r b$ mRNA expression. Additionally, the relative phosphorylation of Ser608, the main phosphorylation site responsible for $\mathrm{Rb}$ inactivation, significantly decreased in response to $20 \mathrm{~h}$ of anoxia in nuclear fractions. Interestingly, the relative acetylation of nuclear $\mathrm{Rb}$, thought to prevent phosphorylation, was correspondingly found to increase after $5 \mathrm{~h}$ anoxia. This could suggest a mechanism where $\mathrm{Rb}$ acetylation at $5 \mathrm{~h}$ of anoxia reduces overall phosphorylation of $\mathrm{Rb}$ at $20 \mathrm{~h}$ of anoxia, allowing $\mathrm{Rb}$ to remain in its active state. The overall expression of p130 increased in both total and nuclear fractions, correlating with an increase in $p 130 \mathrm{mRNA}$ expression. Although protein expression, posttranslation modification and cellular localization provides an indication of the possible role of each protein, a novel in vitro technique was developed for this thesis to distinguish between the types of DNA-binding complex that assembles during anoxia.

The development of the DNA-bound protein complex ELISA method outlined in this chapter was used to evaluate the composition and transcriptional activity of DNA binding complexes (see Appendix J for experimental validation and development). The development of this technique was crucial since the use of traditional co-I $P$ protocols for the evaluation of E2F complexes fail to properly assess the state of cellular proliferation since complexes exist in a free non-DNA bound state. The immunoprecipitation of these 
individual proteins, or those bound in complex, would yield irrelevant results as there is no indication to whether these complexes have any influence on transcriptional activity; positive results would also be diluted by co-precipitation of inactive, free/unbound, protein. Traditional analyses of transcriptionally active transcription factor complexes utilize co-immunoprecipitation and chromatin immunoprecipitation, the cross-linking of proteins to each other and to the surrounding DNA (Giovanni et al., 2001). This technique, although suitable for well-characterized animal models, is extremely difficult to validate for species that are not genome sequenced (the turtle was unsequenced at the time of this study). The inability to determine the DNA sequence to which its bound (no genomic information available) and the ability of this technique to generate a high degree of false positives (the crosslinking of near-neighbor proteins, not part of the target complex) makes chromatin immunoprecitation a costly alternative to the outlined method of DNA-bound protein complex ELISA.

Results from the DNA-bound protein complex ELISA experiments suggest that the liver is progressing through a state of $G_{1}$ arrest during anoxia (Figure 4.14). As mentioned earlier, ELISA experiments indicated that E2F1 DNA-binding decreased while E2F4 binding increased in response to $20 \mathrm{~h}$ of anoxia. This indicated what would seem to be a complete switch to repressive E2F bound to DNA. However, secondary binding proteins (proteins bound to the Rb:p130/E2F complex) displayed an increase in HDAC4 and Suv39H1 binding after $20 \mathrm{~h}$ anoxia. Due to the function of these proteins, these results could be indicative of local DNA condensation around the E2F DNA binding site. The condensation of DNA around the E2F promoter is highly likely as this promoter site is in close proximity to genes involved with DNA replication (pcna, cyclin 
A, geminin, $c d t 1$ ), a process known to be suppressed during hypometabolism (Bracken et al., 2004). Of particular interest is the binding of either $\mathrm{Rb}$ or $\mathrm{p} 130$ to the $\mathrm{E} 2 \mathrm{~F}$ transcription factor, as this is the mechanism widely understood to be the critical difference between $\mathrm{G}_{1}$ and $\mathrm{G}_{0}$ arrest. Similar to other models of anoxia-induced cell cycle arrest, the turtle displayed an increase in $\mathrm{Rb}$ complex formation after $20 \mathrm{~h}$ anoxia, while no significant complex formation was detected for p130. Given these results, it would seem likely that the liver of anoxic turtles may enter into a state of $\mathrm{G}_{1}$ arrest after $20 \mathrm{~h}$ of anoxia, a similar mechanism as seen in hypoxia tolerant organisms, such as C. elegans and D. melanogaster (Kops et al., 2002; Douglas and Haddad, 2003). Given the small, yet significant, increases in p130 mRNA and protein after $20 \mathrm{~h}$ anoxia, it could also be hypothesized that a state of $\mathrm{G}_{1}$ arrest is a transitional state as the cell progresses to a long term state of quiescence $\left(\mathrm{G}_{0}\right)$ if anoxia exposure is prolonged to days or weeks.

\subsubsection{Specific mechanisms of cell cycle arrest in kidney tissue}

Interestingly, the analysis of the same proteins and processes in kidney gave similar results to those detected in liver. However, different from liver, it appears that kidney may undergo a state of cell cycle arrest as early as $5 \mathrm{~h}$ of anoxia. Overall, the expression of E2F1 and E2F4 show similar expression patterns to that of liver tissue. After $20 \mathrm{~h}$ of anoxia, E2F1 significantly decreased in both total and nuclear fractions, whereas E2F4 increased in the same fractions (Figures 4.3 and 4.4). Additionally, transcript levels of $e 2 f 4$ increased with protein expression after $20 \mathrm{~h}$ anoxia (Figure 4.17). Interestingly, the DNA binding ability of E2F1 decreased after $5 \mathrm{~h}$ of anoxia, corresponding to an increase in E2F4 DNA binding (Figure 4.13). The reduction in E2F1 
transcriptional activity was further validated with the demonstration of a decrease in pcna expression after $20 \mathrm{~h}$ of anoxia (Figure 4.15). Similar to results from liver, the protein expression and post-translational modifications of $\mathrm{Rb}$ suggest that this protein is active during anoxia in kidney; increases in nuclear $\mathrm{Rb}$ protein and relative acetylation levels were seen after $5 \mathrm{~h}$ of anoxia with decreases in phosphorylation after $20 \mathrm{~h}$ anoxia (Figure 4.5 and 4.6). However, unlike liver, there was a stronger and earlier increase in p130 protein and mRNA expression (Figure 4.7, 4.8 and 4.17). The expression of p130 in total and nuclear fractions was found to significantly increase after $5 \mathrm{~h}$ of anoxia, correlating with an increase in mRNA expression.

Similar to liver, results from the DNA-bound protein complex ELISA experiments suggest that cells of the kidney enter into a state of overall $\mathrm{G}_{1}$ arrest during anoxia exposure. However unlike liver tissue, results also suggest that entry into $\mathrm{G}_{1}$ arrest is temporary and is beginning a transition into sustained quiescence $\left(\mathrm{G}_{0}\right)$ within $20 \mathrm{~h}$. Analysis of the DNA-binding complex during early anoxia $(5 \mathrm{~h})$ displayed an increase in proteins that comprise a $\mathrm{G}_{1}$ arrest complex; including Rb, E2F4, HDAC4 and Suv39H1 (Figure 4.14). Interestingly, later stages of anoxia showed an increased presence of p130 in the arrest complex. It can be hypothesized that the increased presence of both $\mathrm{Rb}$ and p130 during later stages of anoxia could be an indication that cells from kidney may either comprise of two populations of cell-cycle arrest mechanisms (either $\mathrm{G}_{1}$ or $\mathrm{G}_{0}$ ), or transition through $G_{1}$ arrest in early stages of anoxia towards a sustained quiescence $G_{0}$ for prolonged periods of arrest. This latter mechanism makes sense; it is well documented that cells exposed to various environmental stresses will respond by arresting cells at the R-point transition (also known as $\mathrm{G}_{1}$ arrest) (Chiu et al., 2011). Additionally, $\mathrm{G}_{1}$ arrest at 
the R-point transition is necessary for entry into quiescence (Liu et al., 2007). Therefore, cell populations within the kidney of anoxic $T$. scripta elegans may appear to establish $\mathrm{G}_{1}$ arrest during early anoxia, while undergoing a transition to reversible quiescence $\left(\mathrm{G}_{0}\right)$ for long term suppression of the cell cycle.

\subsubsection{Specific mechanisms of cell cycle arrest in white skeletal muscle}

Although both liver and kidney actively regulate cellular proliferation and utilize mechanisms of cell cycle arrest in response to anoxia exposure, white skeletal muscle appears to forego these specific regulatory mechanisms. Since skeletal muscle is primarily senescent, with irreversible mechanisms of cell cycle arrest already in place in myocytes, perhaps the small alterations in $\mathrm{Rb}$ and $\mathrm{E} 2 \mathrm{~F}$ regulation are needed to utilize specific pathways of the cell cycle without undergoing division. Several studies have documented that cellular hypertrophy, or growth without division, is an adaptive response to various physiological simuli in postmitotic muscle. These tissues typically display transient and selective transcriptional activity of E2F1, leading to the increased expression of a subset of genes necessary for muscle maintenance without cellular division. Such genes typically include those involved with DNA repair (including PCNA), protein synthesis, mitochondrial function and programmed cell death (Shin et al., 1995; Al-Musawi et al., 2011). Further study is needed to explore the functional noncell cycle related roles of $\mathrm{Rb} / \mathrm{E} 2 \mathrm{~F}$ in turtle skeletal muscle during anoxia. 


\subsubsection{Conclusion}

In conclusion, the present study builds upon the specific $\mathrm{G}_{1} / \mathrm{G}_{0}$ regulatory mechanisms previously presented in Chapter 3. Previous results from Chapter 2 and Chapter 3 indicated a general regulation of critical cell cycle components within the $\mathrm{G}_{1} / \mathrm{G}_{0}$ phases of the cell cycle, in addition to the regulation of signaling cascades (PI3K/Akt and ATM/ATR) known to regulate cell cycle progression. The data in this current chapter successfully identified the phase-specific cell cycle arrest mechanism concerning the Rb/E2F DNA-binding complex. Results indicate that both kidney and liver enter into a $G_{1}$ arrest during anoxia. Interestingly, kidney established a state of $G_{1}$ arrest with $5 \mathrm{~h}$ of anoxia exposure, and appeared to be transitioning to a sustainable $\mathrm{G}_{0}$ arrest by $20 \mathrm{~h}$ anoxia. However, it appears that liver $\mathrm{G}_{1}$ arrest was not established until 20 $\mathrm{h}$ anoxia. Since mRNA and protein levels of components associated with $\mathrm{G}_{\mathrm{o}}$ arrest had actually increased after $20 \mathrm{~h}$ of anoxia exposure, as in kidney, it is possible that liver tissue would also transition to $\mathrm{G}_{0}$ arrest upon prolonged exposure to anoxia. A postponed arrest of the cell cycle may be attributed to the lower proliferative potential of liver, compared to that of the kidney. Future studies will need to explore the regulation of the cell cycle after longer exposures to anoxia to definitively confirm whether hepatocytes are able to transition into $\mathrm{G}_{0}$ arrest. 


\section{References}

Al-Musawi, S.L., Lock, F., Simbi, B.H., Bayol, S.A., and Stickland, N.C. 2011. Muscle specific differences in the regulation of myogenic differentiation in chickens genetically selected for divergent growth rates. Differentiation, 82(3), 127-135.

Alvaro-Blanco, J.,Martinez-Gac, L., Calonge, E., Rodriguez-Martinez, M., MolinaPrivado, I., Redondo, J.M., Alcami, J., Flemington, E.K., Campanero, M.R. 2009. A novel factor distinct from E2F mediates C-MYC promoter activation through its E2F element during exit from quiescence. Carcinogenesis, 30, 440-448.

Andersen, S.U., Algreen-Petersen, R.G., Hoedl, M., Jurkiewicz, A., Cvitanich, C., Braunschweig, U., Schauser, L., Oh, S., Twell, T., Jensen, E.O. 2007. The conserved cysteine-rich domain of a tesmin/TSO1-like protein binds zinc in vitro and TSO1 is required for both male and female fertility in Arabidopsis thaliana. J. Exp. Bot., 58, 3657-3670.

Bernards, R. 1997. E2F: a nodal point in cell cycle regulation. Biochim. Biophys. Acta., $1333,33-40$.

Bracken, A., Ciro, M., Cocito, A., Helin, K. 2004. E2F target genes: unraveling the biology. Trends Biochem. Sci., 29, 409-417.

Chiu, J., Tactacan, C.M., Shi-Xiong, T., Lin, R.C.Y., Wouters, M.A., and Dawes, I.W. 2011. Cell cycle sensing of oxidative stress in Saccharomyces cerevisiae by oxidation of a specific cysteine residue in the transcription factor Swi6p. J. Biol. Chem. 286(7), 5204-5214.

DeGregori, J. 2004. The Rb network. J. Cell Sci., 117, 3411-3413.

Delston, R., Harbour, J. 2006. Rb at the interface between cell cycle and apoptotic decisions. Curr. Mol. Med., 6, 713-718.

Du, W., Pogoriler, J. 2006. Retinoblastoma family genes. Oncogene, 25, 5190-5200.

Ferreira, R., Naguibneva, I., Pritchard, L., Ait-Si-Ali, S., Harel-Bellan, A. 2001. The $\mathrm{Rb}$ /chromatin connection and epigenetic control: opinion. Oncogene, 20, 31283133.

Fischle, W., Tseng, B., Holger. L., Dorman. N., Beatrix, M., Ueberheide, B., Garcia, J., Shabanowitz, D., Hunt. F., Hironori, F., Allis, C. 2005. Regulation of HP1chromatin binding by histone $\mathrm{H} 3$ methylation and phosphorylation. Nature, 438, 1116-1122.

Giovanni, B., Du, W. and T.L. Orr-Weaver. 2001. DNA replication control through the interaction of E2F-RB and the origin recognition complex. Nature Cell Biol. 3: 289-295.

Giacinti, C., Giordano, A. 2006. RB and cell cycle progression. Oncogene, 25, 52205227.

Grana, X., Garriga, J., Mayol, X. 1998. Role of the retinoblastoma protein family, pRB, p107 and p130 in the negative control of cell growth. Oncogene, 17, 3365-3383. 
Guardavaccaro, D., Pagano, M. 2006. Stabilizers and destabilizers controlling cell cycle oscillators. Mol. Cell., 22, 1-4.

Hansen, K., Farkas, T., Lukas, J., Holm, K., Ronnstrand, L., Bartek, J. 2001. Phosphorylation-dependent and-independent functions of p130 cooperate to evoke a sustained $\mathrm{G}_{1}$ block. EMBO J., 20, 422-432.

Harbour, J.W., and Dean, D.C. 2000. The Rb/E2F pathway: expanding roles and emerging paradigms. Genes Dev., 14, 2393-2409.

Hijmans, P.W., Voorhoeve, P.M., Beijersbergen, R.L., van't Veer, L.J., and Bernards, R. 1995. E2F-5, a new E2F family member that interacts with p130 in vivo. Mol. Cell. Biol., 15, 3082-3089.

Leng, X., Noble, M., Adams, P., Qin, J., Harper, J. 2002. Reversal of growth suppression by p107 via direct phosphorylation by cyclin D1/cyclin-dependent kinase 4. Mol. Cell Biol., 22, 2242-2254.

Lewis, P.W., Beall, E.L., Fleischer, T.C., Georlette, D., Link, A.J., Botchan, M.R. 2004. Identification of a Drosophila Myb-E2F2/RBF transcriptional repressor complex. Genes Dev., 18, 2929-2940

Litovchick, L., Sadasivam, S., Florens, L., Zhu, X., Swanson, S., Velmurugan, S., Chen, R., Washburn, M., Liu, W., DeCaprio, J. 2007. Evolutionarily conserved multisubunit RBL2/p130 and E2F4 protein complex represses human cell cycledependent genes in quiescence. Mol. Cell., 26, 539-551.

Litovchick, L., Chestukhin, A., DeCaprio, J. 2004. Glycogen synthase kinase 3 phosphylates RbL2/p130 during quiescence. Mol. Cell. Biol., 24, 8970-8980

Liu, H., Adler, A.S., Segal, E., and Chang, H.Y. 2007. A transcriptional program mediating entry into cellular quiescence. PLoS Genet., 3(6), e91.

Lu, J., Gilbert, D. 2007. Proliferation-dependent and cell cycle-regulated transcription of mouse preicentric heterochromatin. J. Cell Biol., 179, 411-421.

Lundberg, A., Weinberg, R. 1999. Control of the cell cycle and apoptosis. Eur. J. Cancer., 35, 1886-1894.

Luo, R.X., Postigo, A.A. and D.C. Dean. 1998. Rb interacts with histone deacetylase to repress transcription. Cell, 92(4), 463-473.

Kingston, R., Narlikar, G. 1999. ATP-dependent remodeling and acetylation as regulators of chromatin fluidity. Genes Dev., 13, 2338-2352.

Kops, G., Medema, R., Glassford, J., Essers, M., Dijkers, P., Coffer, P., Lam, E., Burgering, B. 2002. Control of cell cycle exit and entry by protein kinase Bregulated forkhead transcription factors. Mol. Cell. Biol., 22, 2025-2036.

Muchardt, C., Yaniv, M. 1999. The mammalian SWI/SNF complex and the control of cell growth. J. Mol. Biol., 293, 187-198.

Narita, M., Nunez, S., Heard, E., Narita, M., Lin, A., Hearn, S., Spector, D., Hannon, G., Lowe, S. 2003. Rb-mediated heterochromatin formation and silencing of E2F target genes during senescence. Cell, 113, 703-716. 
Nevins, J.R. 1998. Toward an understanding of the functional complexity of the E2F and retinoblastoma families. Cell Growth Differ., 9, 585-593.

Pilkinton, M., Sandoval, R., Barrett, K., Tian, X., Colamonici, O. 2007. Mip/LIN-9 can inhibit proliferation independent of the pocket proteins. Blood Cell. Mol. Dis., 39, 272-277.

Schmitz, N., Hirt, A., Aebi, M., Leibundgut, K. 2006. Limited redundancy in phosphorylation of retinoblastoma tumor suppressor protein by cyclin-dependent kinases in acute lymphoblastic leukemia. Am. J. Pathol., 3, 1074-1079.

Shen, W. 2002. The plant E2F-Rb pathway and epigenetic control. Trends Plant Sci., 7, $1-8$.

Shin, E.K., Shin, A., Paulding, C., Schaffhausen, B., and Yee, A.S. 1995. Multiple change in $\mathrm{E} 2 \mathrm{~F}$ function and regulation occur upon muscle differentiation. Mol. Cell. Biol. 15(4), 2252-2262.

Trimarchi, J., Lees, J. 2002. Sibling rivalry in the E2F family. Mol. Cell Biol., 3, 11-19.

Trojer, P., Li, G., Sims, R., Vaquero, A., Kalakonda, N., Boccuni, P., Lee, D., ErdjumentBromage, H., Tempst, P., Nimer, S., Wang, Y., Reinberg, D. 2007. L3MBTL1, a histone-methylation-dependent chromatin lock. Cell, 129, 915-928.

Trotter, K., Archer, T. 2008. The BRG1 transcription coregulator. Nucl. Recept. Signal., $6,1-12$.

Tyler, J., Kadonaga, J. 1999. The dark side of chromatin remodeling: repressive effects on transcription. Cell, 99, 443-446.

Vairo, G., Livingston, D.M. and D. Ginsberg. 1995. Functional interaction between E2F4 and p130: evidence for distinct mechanisms underlying growth suppression by different retinoblastoma protein family members. Genes Dev., 9, 869-881.

van den Heuvel, S., Dyson, N. 2008. Conserved functions of the pRB and E2F families. Nature Rev. Mol. Cell. Biol., 9, 713-722.

Wong, S., Weber, J.D. 2007. Deacetylation of the retinoblastoma tumour suppressor protein by SIRT1. Biochem J., 207, 451-460.

Zhang, S.H., Dean, D.C. 2001. Rb-mediated chromatin structure regulation and transcriptional repression. Oncogene, 20 3134-3138. 

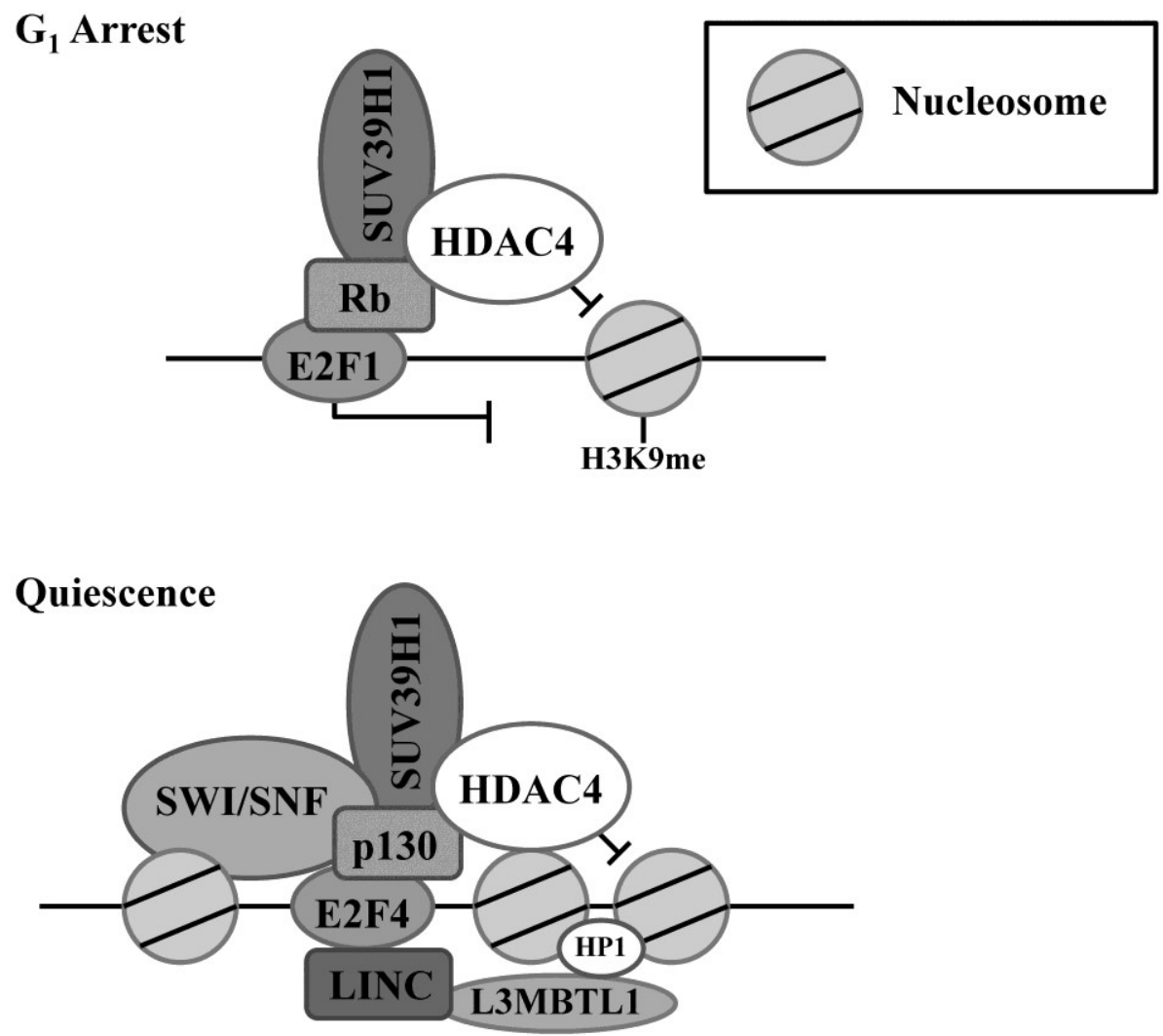

Figure 4.1 The $\mathrm{Rb}$ /E2F pathway. Sequential phosphorylation by kinase complexes Cyclin D1/Cdk4:Cdk6 and Cyclin E1/Cdk2, respectively, causes conformational changes to the Rb structure and release of E2F. The release of E2F is necessary for the expression of S-phases genes. 


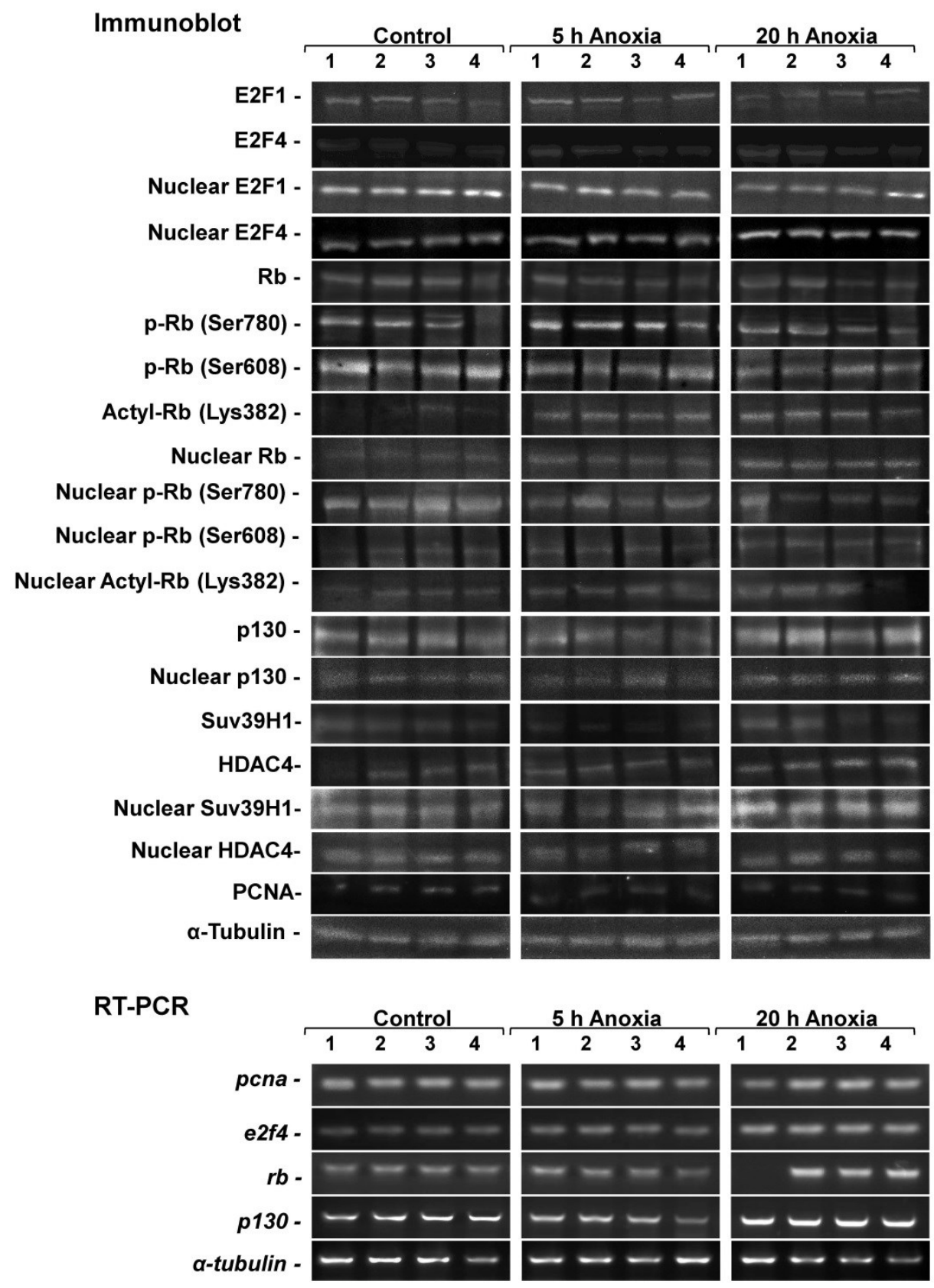

Figure 4.2A Image files for immunoblotting and RT-PCR targets in control and anoxic (5 and $20 \mathrm{~h})$ liver tissue from T. scripta elegans. 


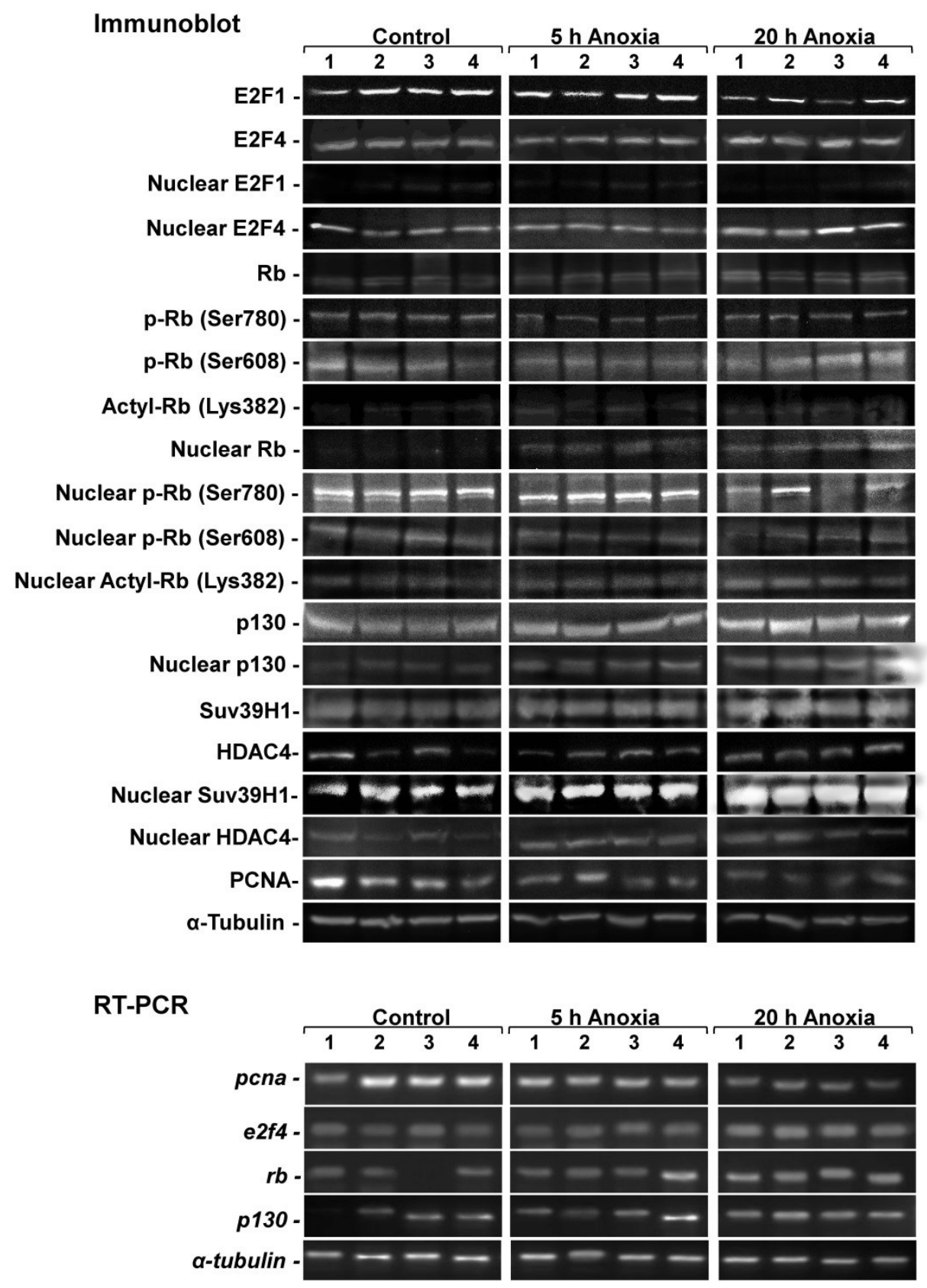

Figure 4.2B Image files for immunoblotting and RT-PCR targets in control and anoxic (5 and $20 \mathrm{~h}$ ) kidney tissue from $T$. scripta elegans. 


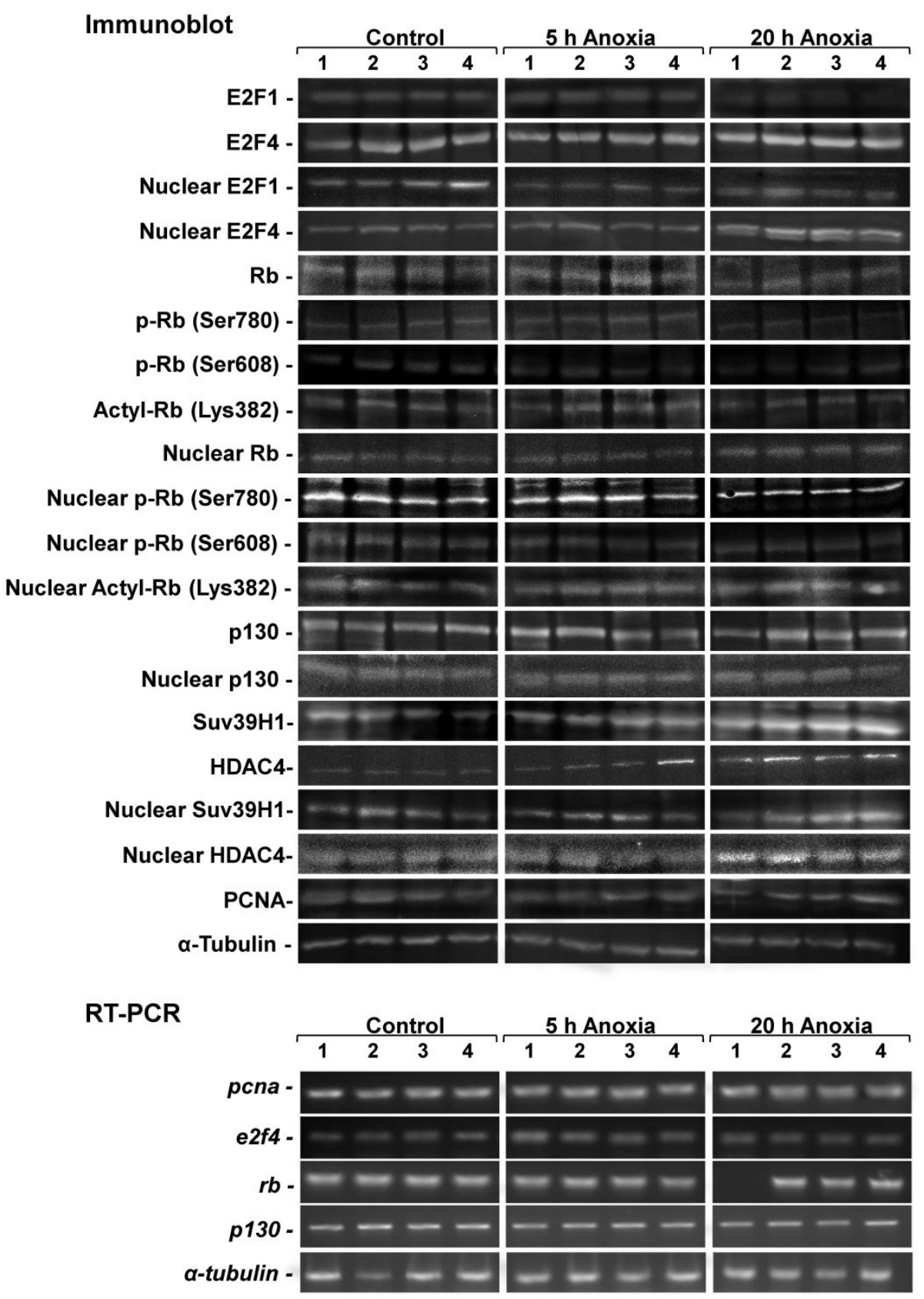

Figure 4.2C Image files for immunoblotting and RT-PCR targets in control and anoxic (5 and $20 \mathrm{~h})$ white skeletal muscle tissue from T. scripta elegans. 
A)

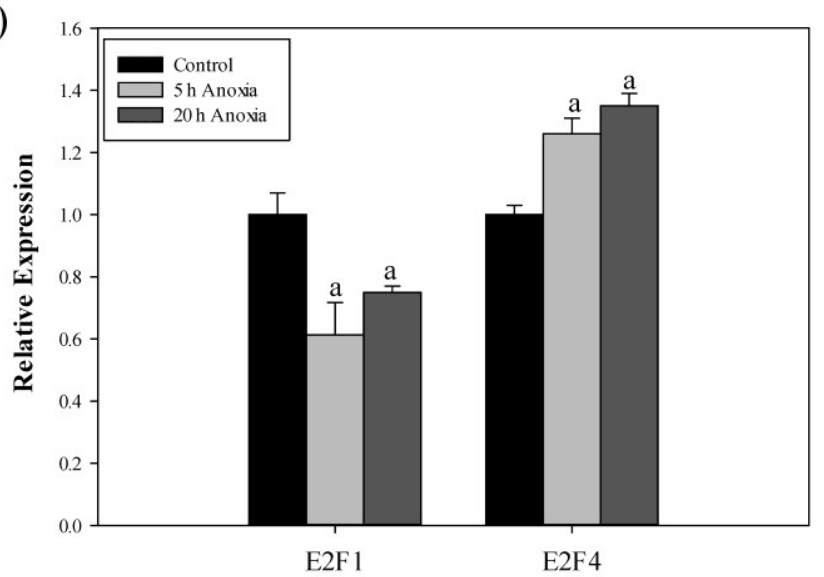

B)

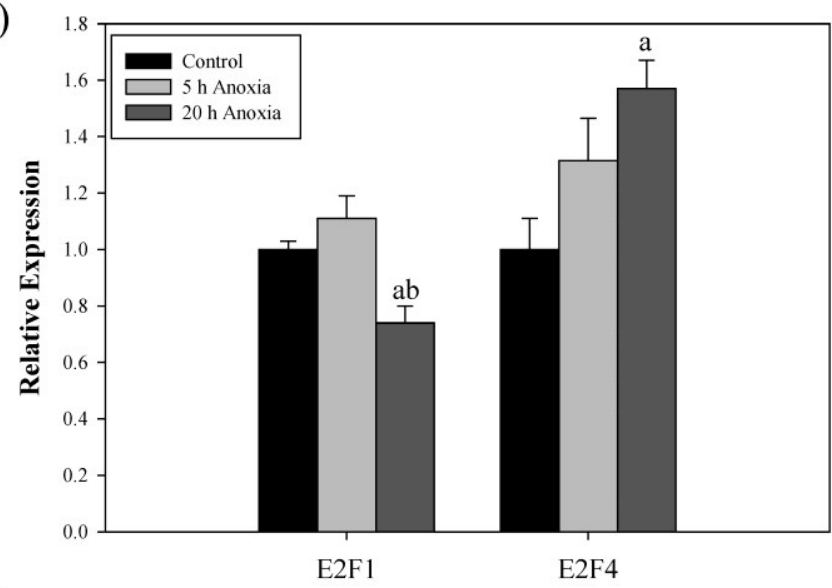

C)

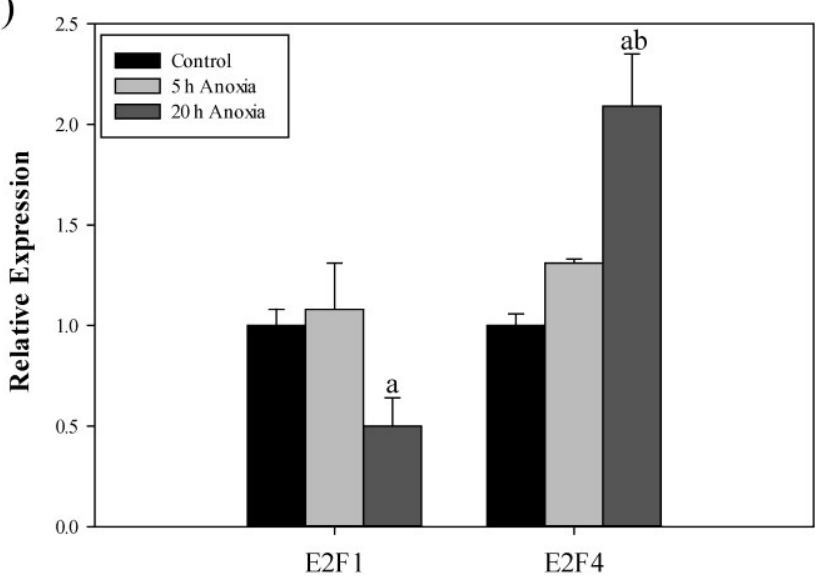

Figure 4.3. Effect of 5 and $20 \mathrm{~h}$ of anoxic submergence on relative protein expression of E2F1 and E2F4 in T. s. elegans. Histograms show normalized expression levels under control and anoxic conditions in (A) liver, (B) kidney and (C) white skeletal muscle tissues. Data are means \pm S.E.M., $n=3-4$ independent trials. a: Significantly different from the corresponding control $(\mathrm{P}<0.05)$; b: significantly different from the $5 \mathrm{~h}$ anoxic value $(\mathrm{P}<0.05)$. 
A)

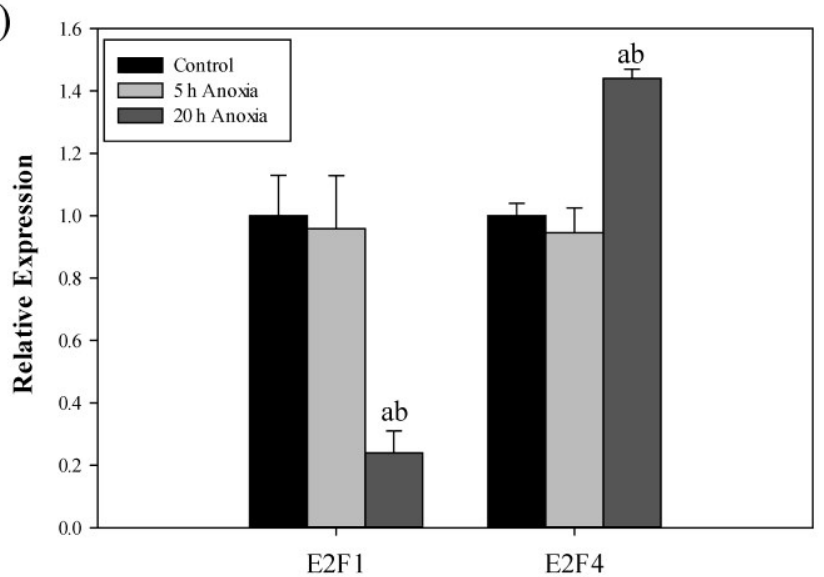

B)

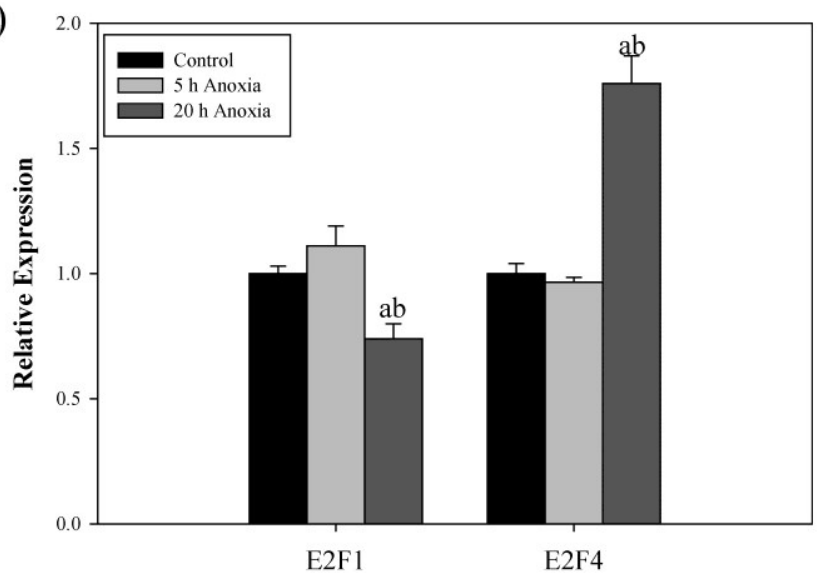

C)

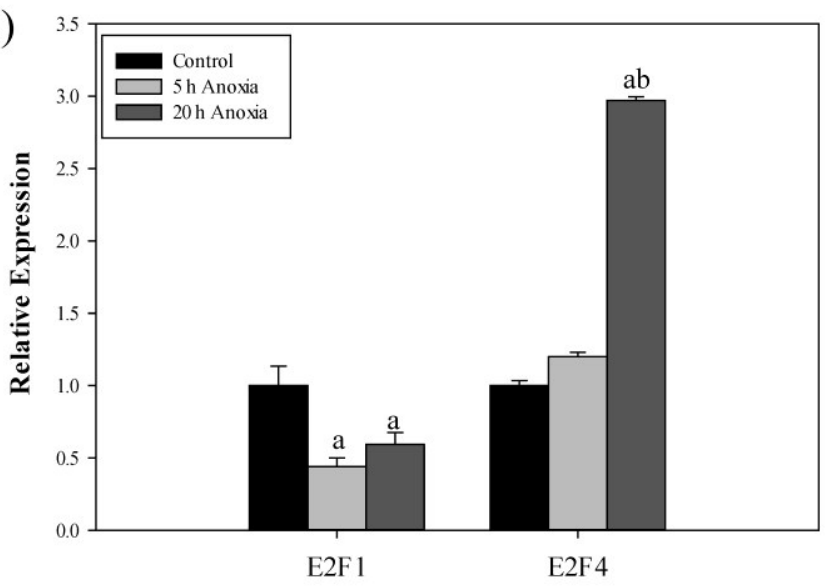

Figure 4.4. Effect of 5 and $20 \mathrm{~h}$ of anoxic submergence on relative nuclear protein expression of E2F1 and $\mathrm{E} 2 \mathrm{~F} 4$ in T. s. elegans. Histograms show normalized expression levels under control and anoxic conditions in (A) liver, (B) kidney and (C) white skeletal muscle tissues. Data are means \pm S.E.M., $n=3-$ 4 independent trials. a: Significantly different from the corresponding control $(\mathrm{P}<0.05)$; b: significantly different from the $5 \mathrm{~h}$ anoxic value $(\mathrm{P}<0.05)$. 
A)

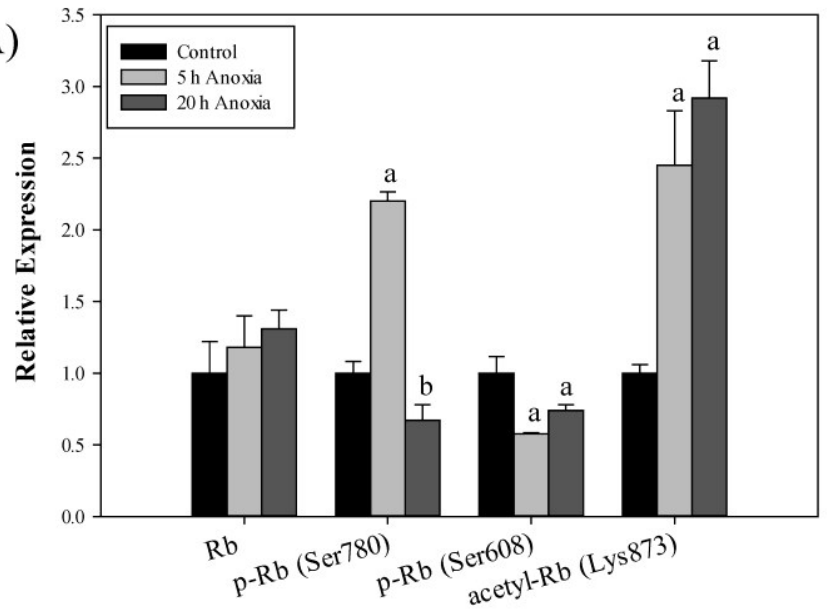

B)

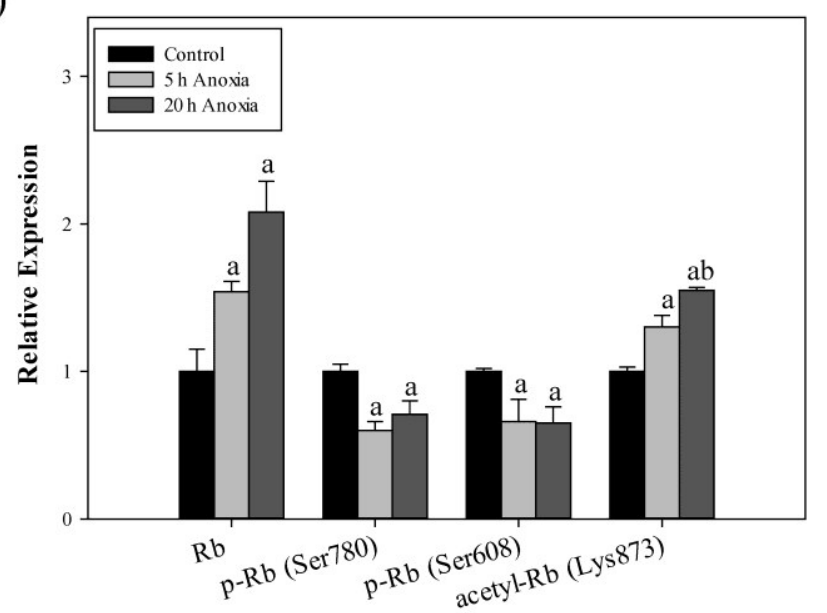

C)

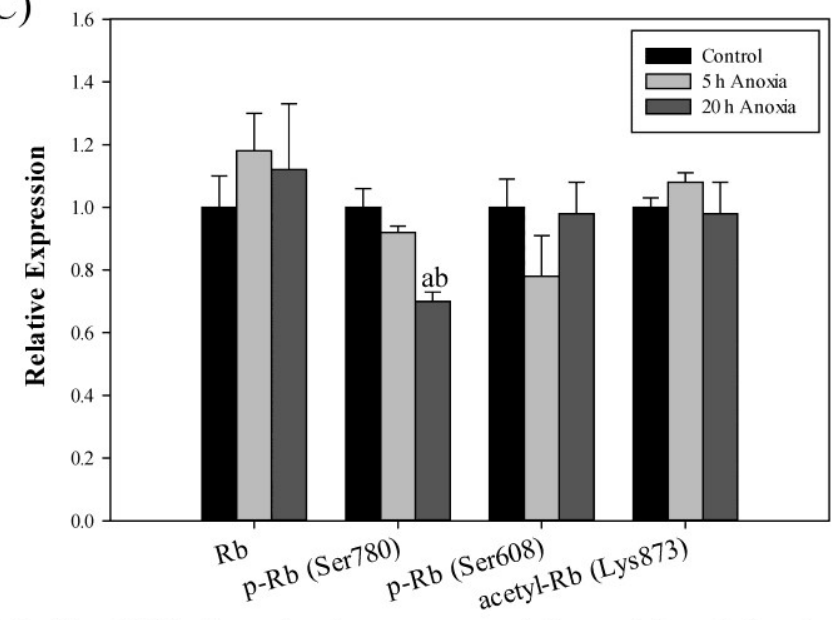

Figure 4.5. Effect of 5 and $20 \mathrm{~h}$ of anoxic submergence on relative protein and phosphorylation levels of $\mathrm{Rb}$ in T. s. elegans. Histograms show normalized expression levels under control and anoxic conditions in (A) liver, (B) kidney and (C) white skeletal muscle tissues. Data are means \pm S.E.M., $n=3-4$ independent trials. a: Significantly different from the corresponding control $(\mathrm{P}<0.05)$; b: significantly different from the $5 \mathrm{~h}$ anoxic value $(\mathrm{P}<0.05)$. 
A)

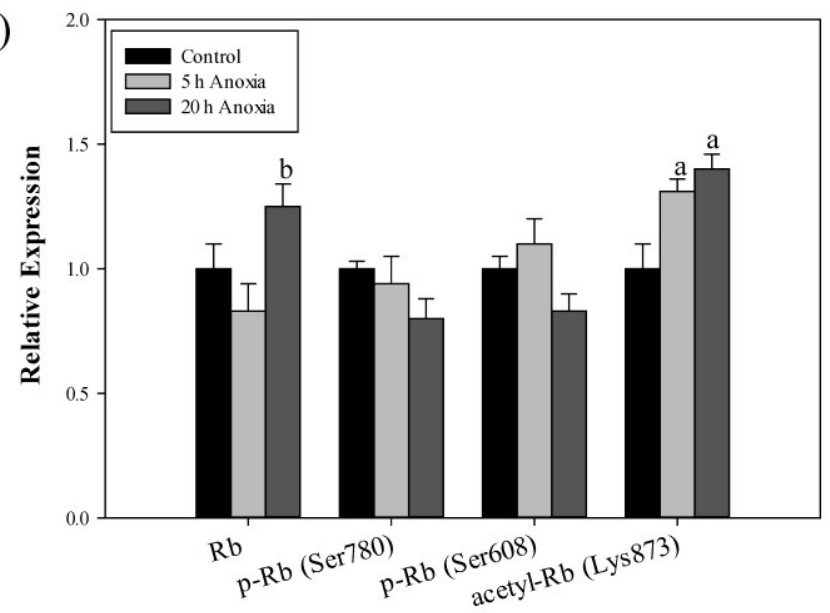

B)

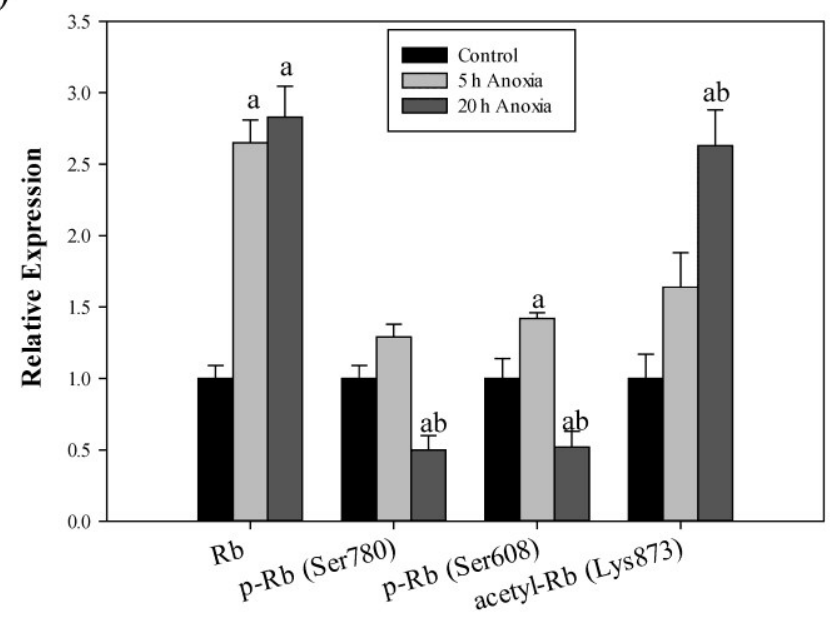

C)

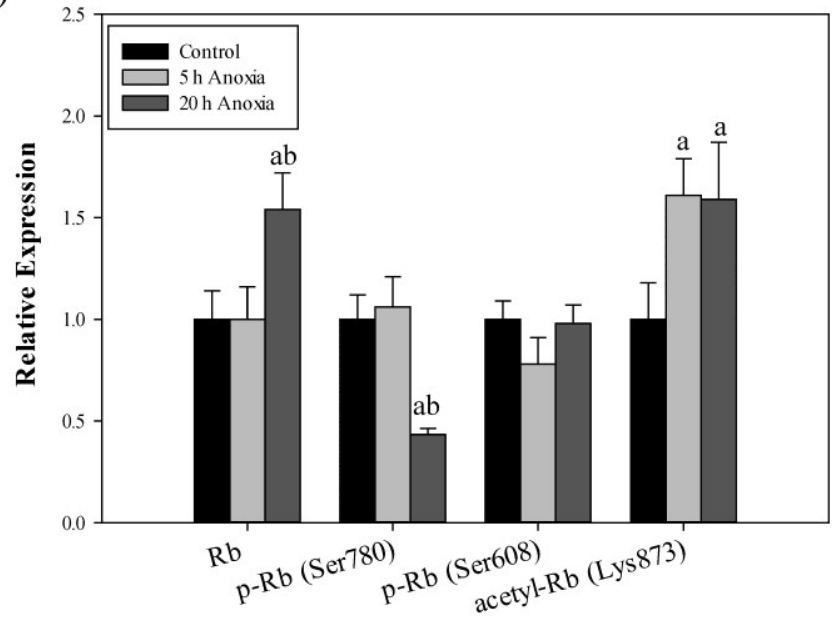

Figure 4.6. Effect of 5 and $20 \mathrm{~h}$ of anoxic submergence on relative nuclear protein and phosphorylation levels of $\mathrm{Rb}$ in T. s. elegans. Histograms show normalized expression levels under control and anoxic conditions in (A) liver, (B) kidney and (C) white skeletal muscle tissues. Data are means \pm S.E.M., $\mathrm{n}=3$ 4 independent trials. a: Significantly different from the corresponding control $(\mathrm{P}<0.05)$; b: significantly different from the $5 \mathrm{~h}$ anoxic value $(\mathrm{P}<0.05)$. 
A)

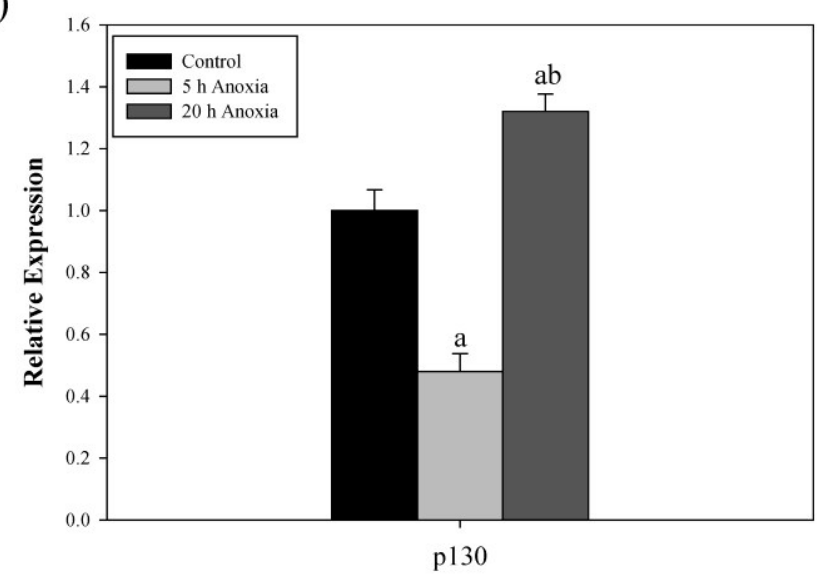

B)

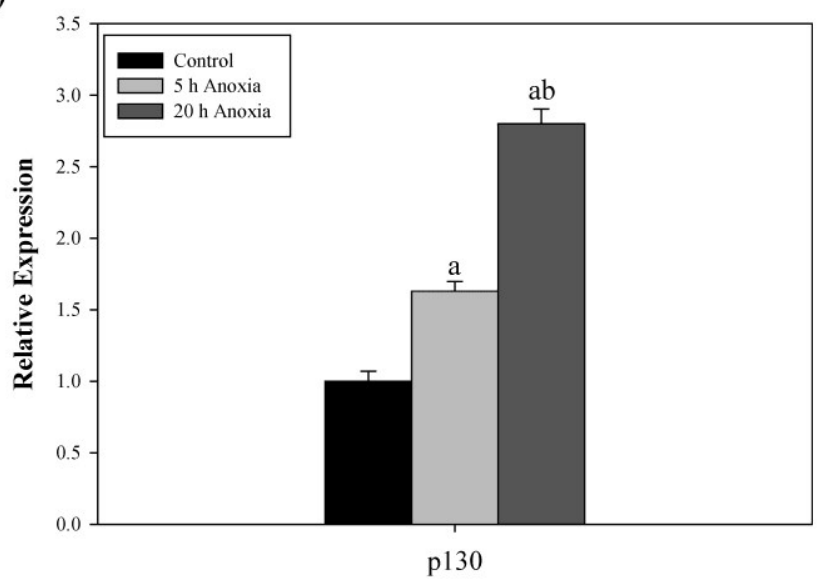

C)

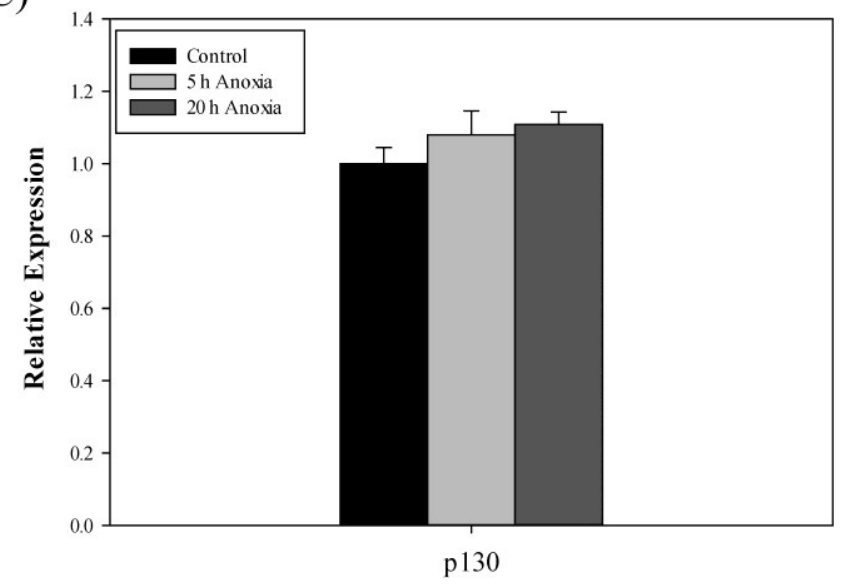

Figure 4.7. Effect of 5 and $20 \mathrm{~h}$ of anoxic submergence on relative protein expression of $\mathrm{p} 130$ in $T$. $s$. elegans. Histograms show normalized expression levels under control and anoxic conditions in (A) liver, (B) kidney and (C) white skeletal muscle tissues. Data are means \pm S.E.M., $n=3-4$ independent trials. a: Significantly different from the corresponding control $(\mathrm{P}<0.05)$; b: significantly different from the $5 \mathrm{~h}$ anoxic value $(\mathrm{P}<0.05)$. 
A)

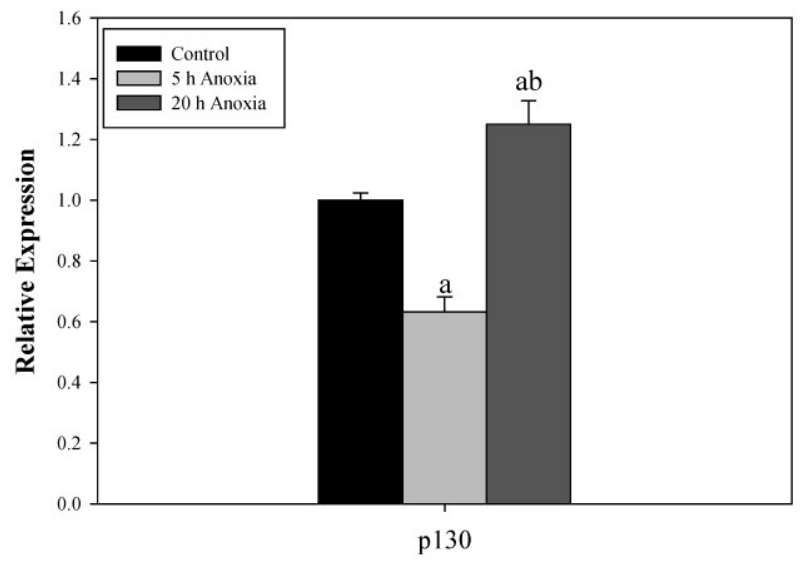

B)

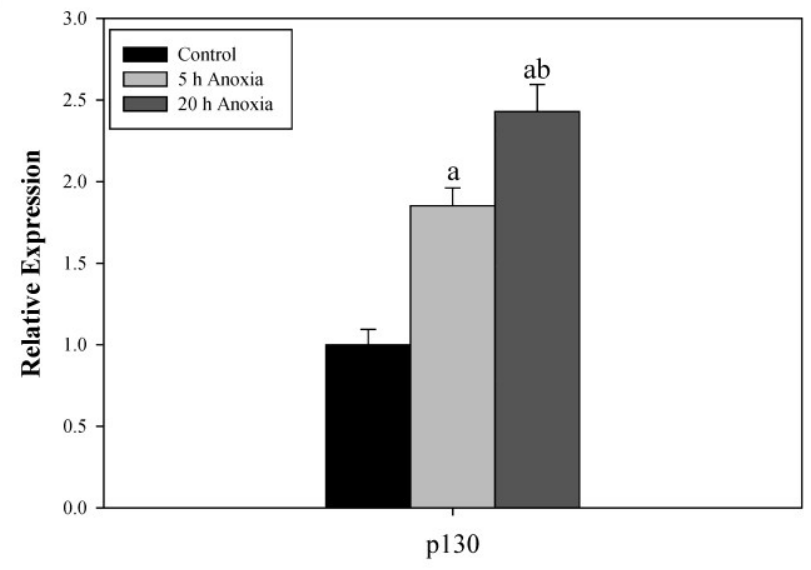

C)

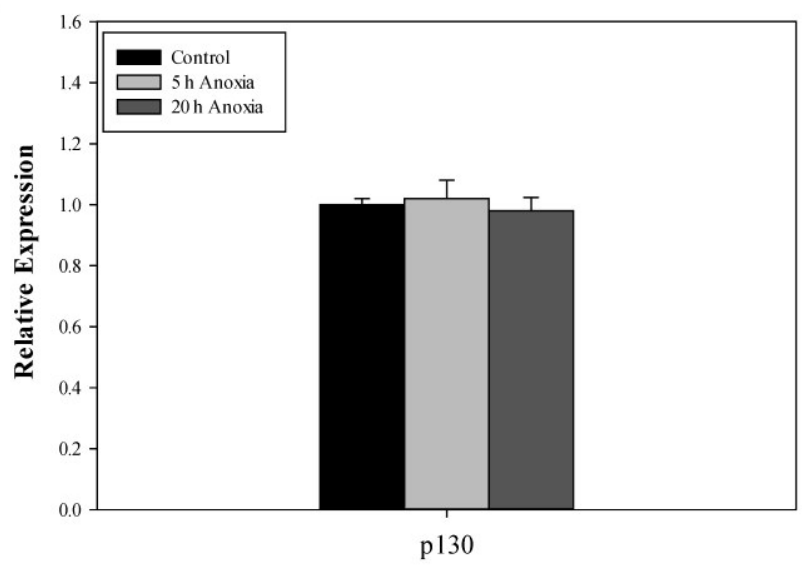

Figure 4.8. Effect of 5 and $20 \mathrm{~h}$ of anoxic submergence on the relative nuclear protein expression of p130 in T. s. elegans. Histograms show normalized expression levels under control and anoxic conditions in (A) liver, (B) kidney and (C) white skeletal muscle tissues. Data are means \pm S.E.M., $n=3-4$ independent trials. a: Significantly different from the corresponding control $(\mathrm{P}<0.05)$; b: significantly different from the $5 \mathrm{~h}$ anoxic value $(\mathrm{P}<0.05)$. 
A)

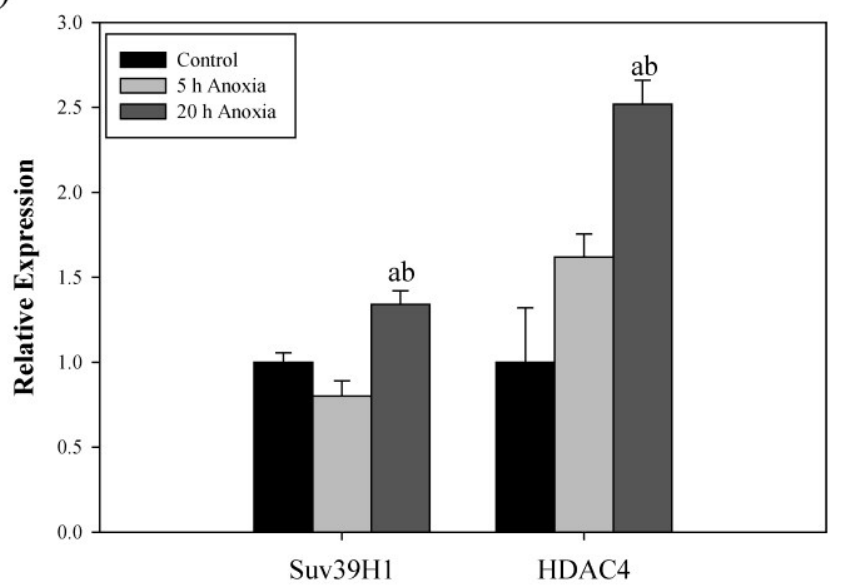

B)

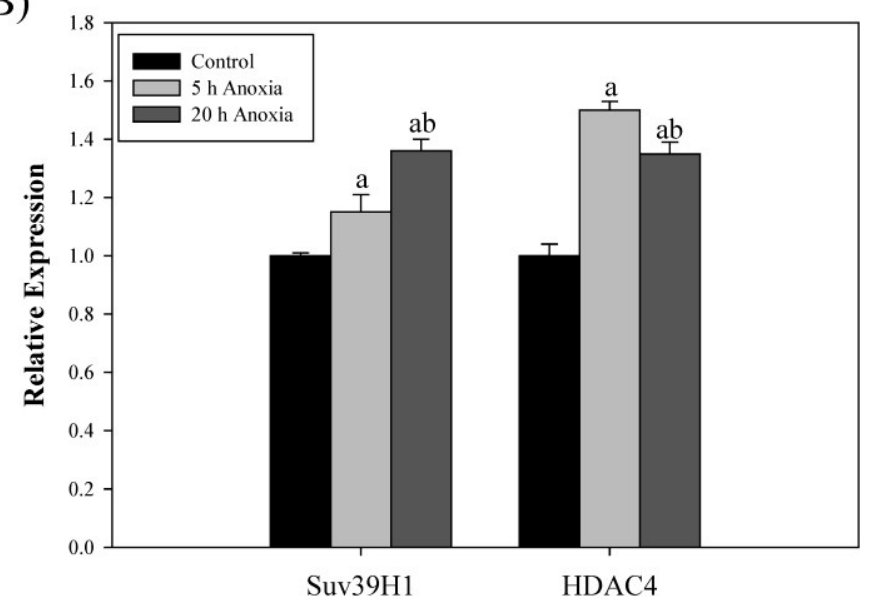

C)

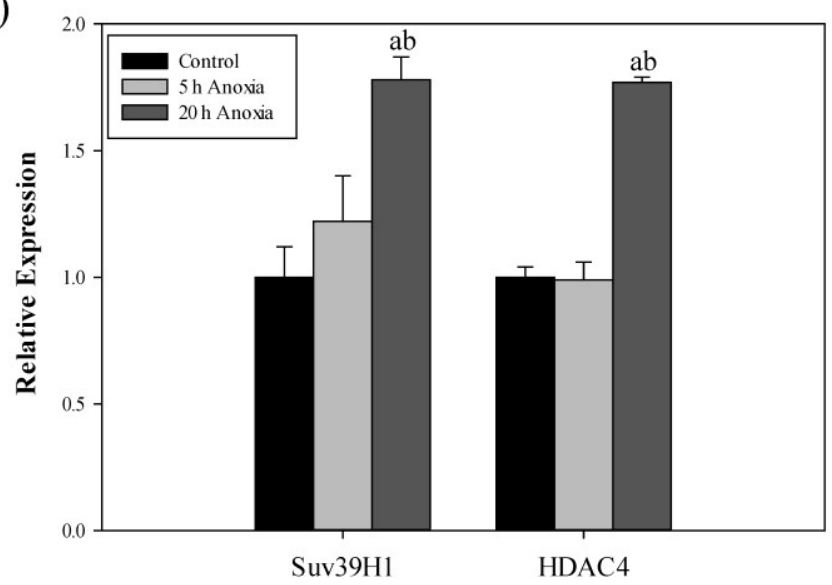

Figure 4.9. Effect of 5 and $20 \mathrm{~h}$ of anoxic submergence on relative protein expression of epigentic modifying protein, Suv29H1 and HDAC4, in T. s. elegans. Histograms show normalized expression levels under control and anoxic conditions in (A) liver, (B) kidney and (C) white skeletal muscle tissues. Data are means \pm S.E.M., $n=3-4$ independent trials. a: Significantly different from the corresponding control $(\mathrm{P}<0.05)$; b: significantly different from the $5 \mathrm{~h}$ anoxic value $(\mathrm{P}<0.05)$. 
A)

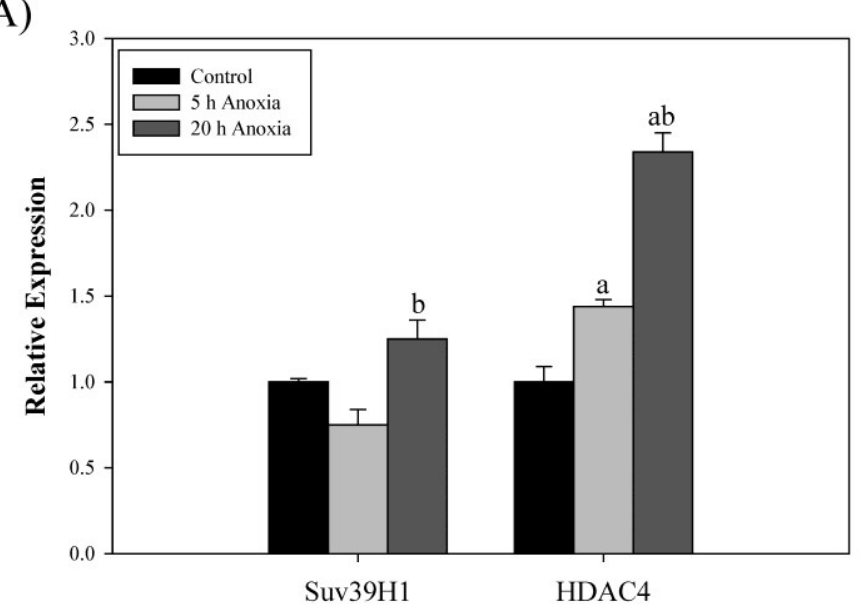

B)

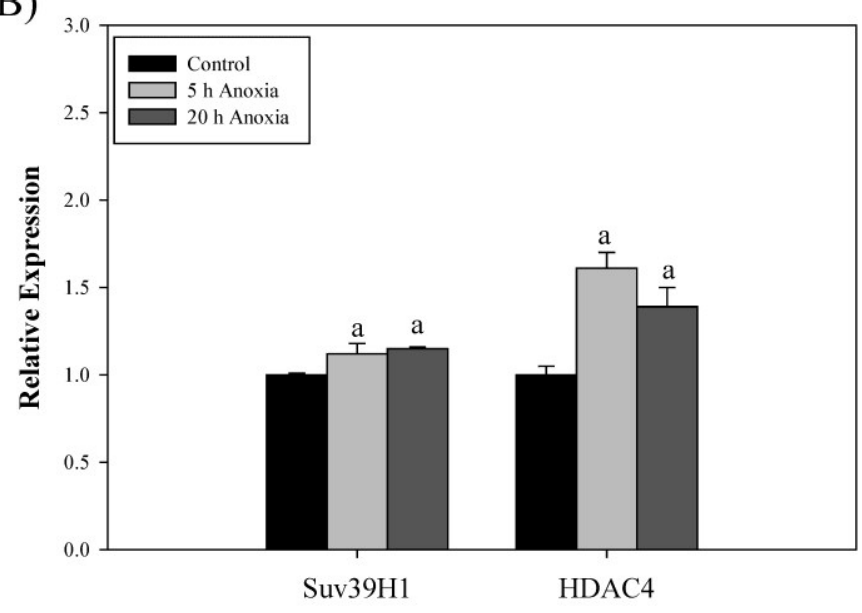

C)

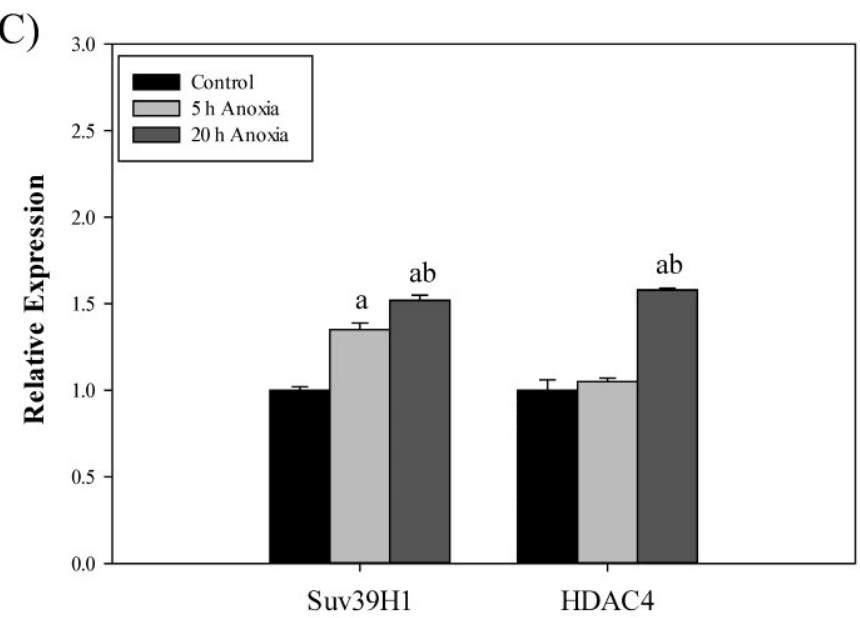

Figure 4.10. Effect of 5 and $20 \mathrm{~h}$ of anoxic submergence on the relative nuclear protein expression of epigentic modifying protein, Suv29H1 and HDAC4, in T. s. elegans. Histograms show normalized expression levels under control and anoxic conditions in (A) liver, (B) kidney and (C) white skeletal muscle tissues. Data are means \pm S.E.M., $n=3-4$ independent trials. a: Significantly different from the corresponding control $(\mathrm{P}<0.05)$; b: significantly different from the $5 \mathrm{~h}$ anoxic value $(\mathrm{P}<0.05)$. 


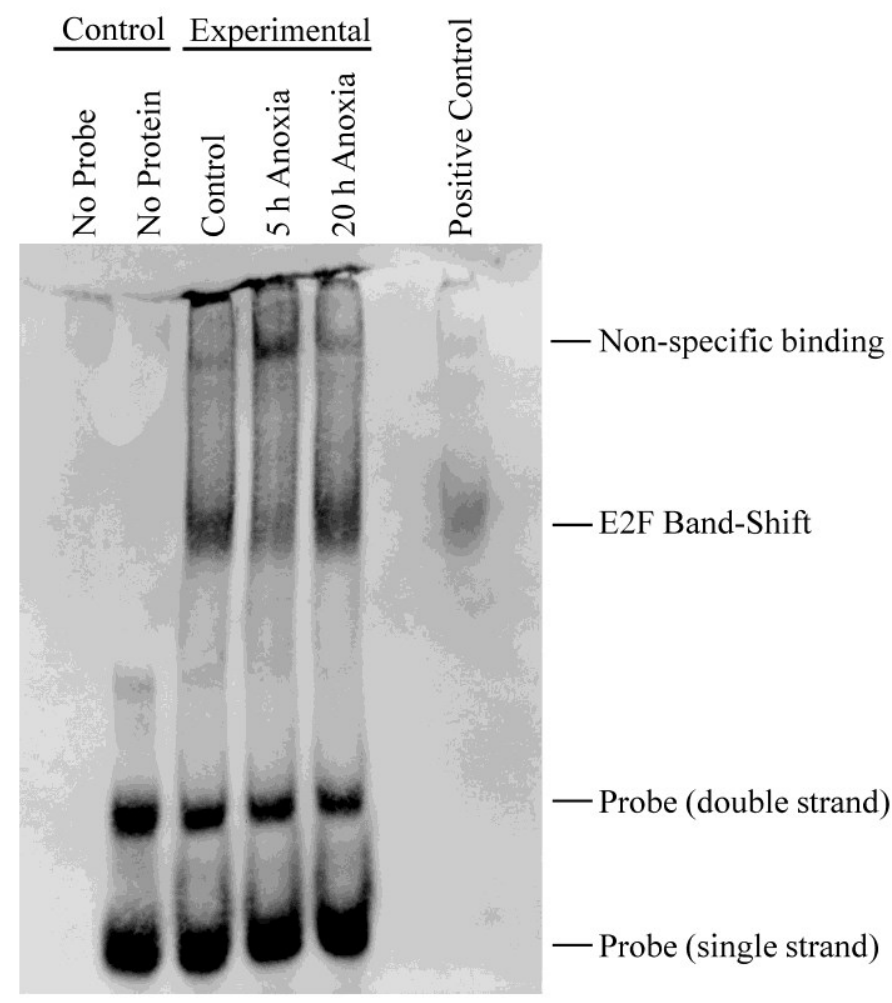

Figure 4.11. Electrophoretic mobility shift assay (EMSA) for the E2F family of transcription factors. Distinct bands, as detected with streptavidin HRP, is indicative of E2F binding as detected in all experimental samples from turtle liver tissues, as well as the commercially available positive control. Negative control lanes include turtle liver proteins without DNA probe (No Probe) and DNA probe without any protein (No Protein). Bands at the bottom of the gel are indicative of single (incompletely annealed) and double stranded biotinylated DNA probe. 


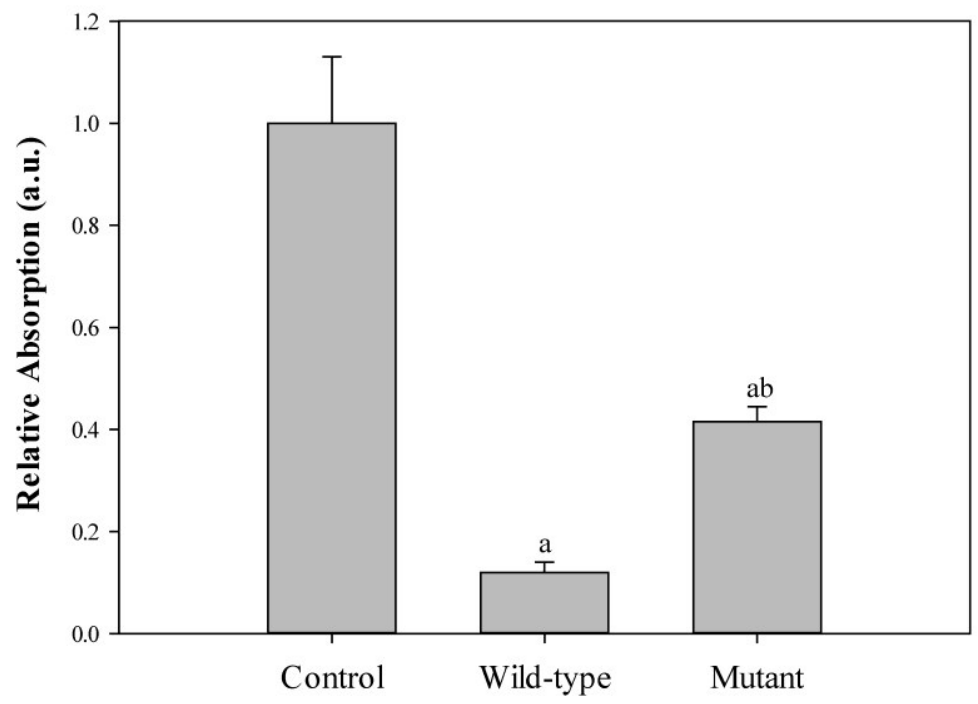

Figure 4.12. Effect of unlabeled DNA probe competition on the relative binding of E2F1 from control liver tissue samples from T. s. elegans. Either unlabeled DNA probe (Wild-type) or an unlabeled DNA probe with a mutated DNA binding site (Mutant) were incubated with total protein in the presence of biotin-labeled probe. Reduced binding indicates successful competition with E2F1 probe binding, indicating probe specificity. Histogram shows normalized relative binding levels for E2F1; data are means \pm SEM. ( $n=3-4$ independent trials on tissue from different animals). Significant differences from the control condition is indicated (a) at $\mathrm{P}<0.05$. 
A)

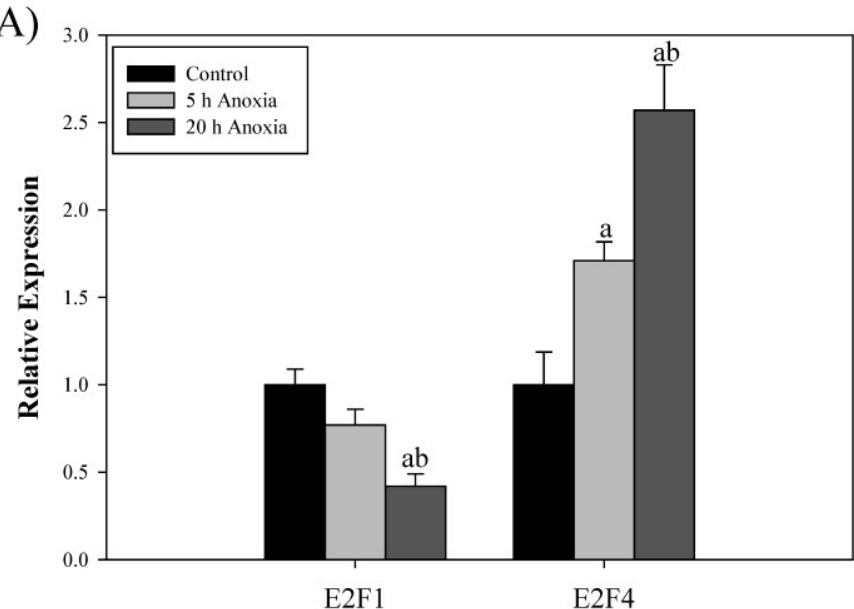

B)
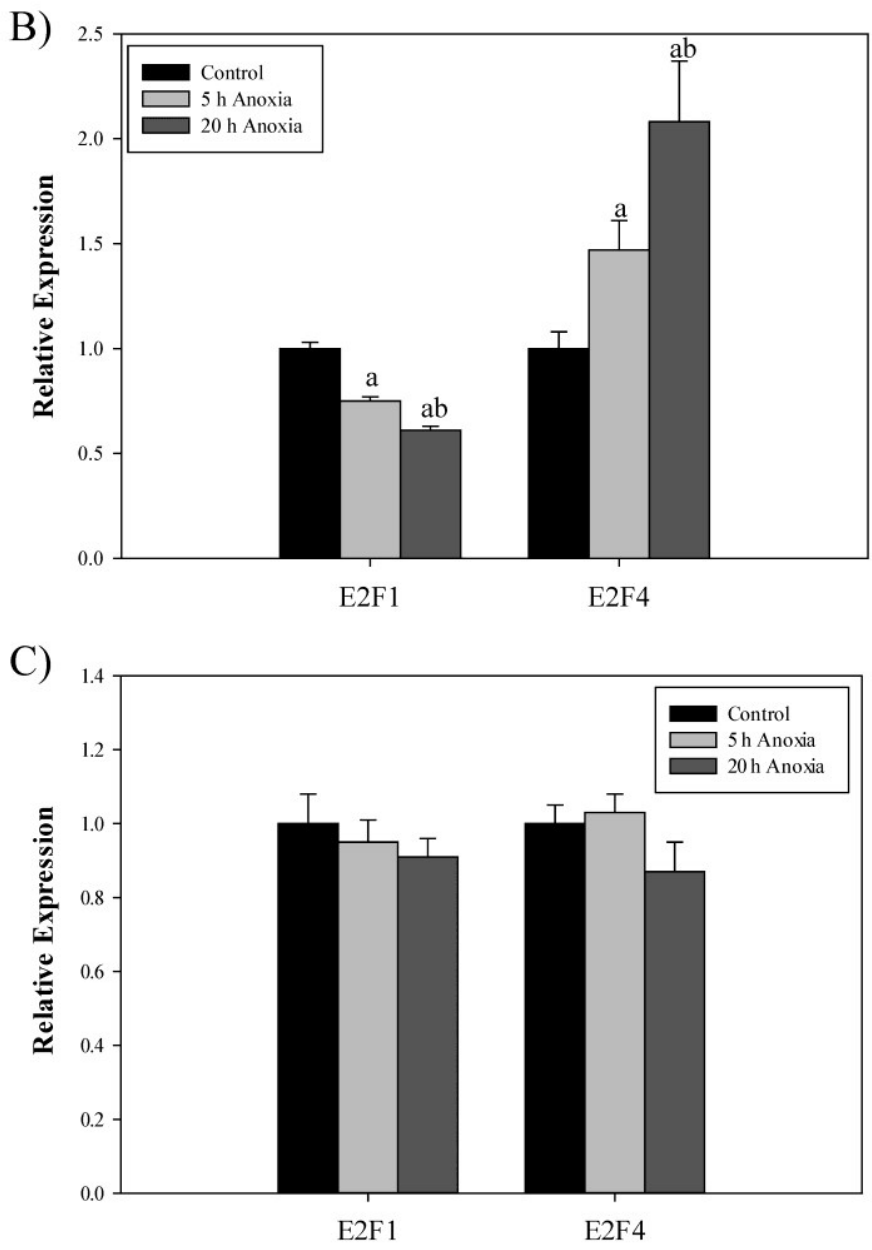

Figure 4.13. Effect of 5 and $20 \mathrm{~h}$ of the relative DNA binding activity of E2F1 and E2F4 in T. s. elegans. Histograms show normalized DNA-binding levels under control and anoxic conditions in (A) liver, (B) kidney and $(C)$ white skeletal muscle tissues. Data are means \pm S.E.M., $n=3-4$ independent trials. a: Significantly different from the corresponding control $(\mathrm{P}<0.05)$; b: significantly different from the $5 \mathrm{~h}$ anoxic value $(\mathrm{P}<0.05)$. 

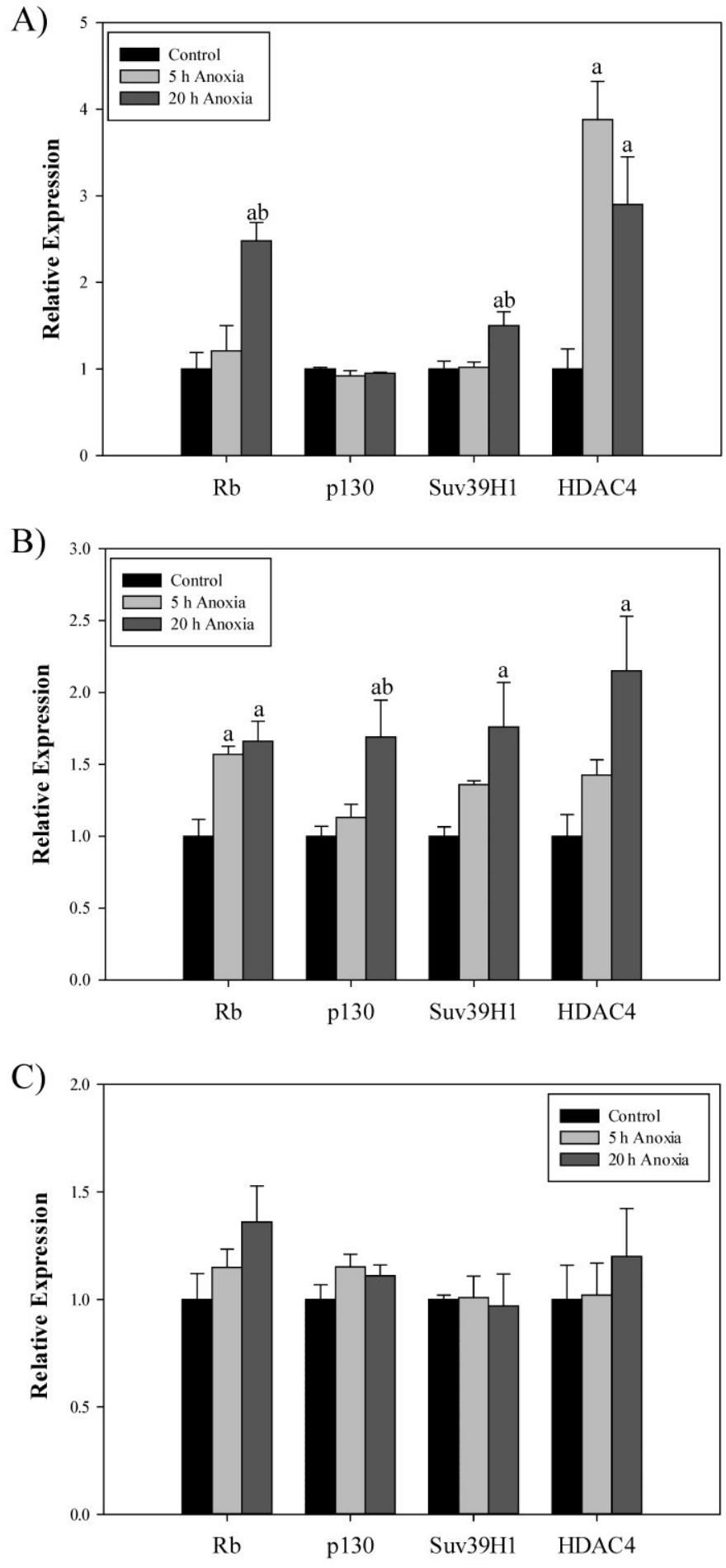

Figure 4.14. Effect of 5 and $20 \mathrm{~h}$ of the relative protein complex composition containing E2F bound to DNA in T. s. elegans. E2F bound proteins include Rb, p130, Suv39H1 and HDAC4. Histograms show normalized DNA-binding levels under control and anoxic conditions in (A) liver, (B) kidney and (C) white skeletal muscle tissues. Data are means \pm S.E.M., $n=3-4$ independent trials. a: Significantly different from the corresponding control $(\mathrm{P}<0.05)$; $\mathrm{b}$ : significantly different from the $5 \mathrm{~h}$ anoxic value $(\mathrm{P}<0.05)$. 
A)

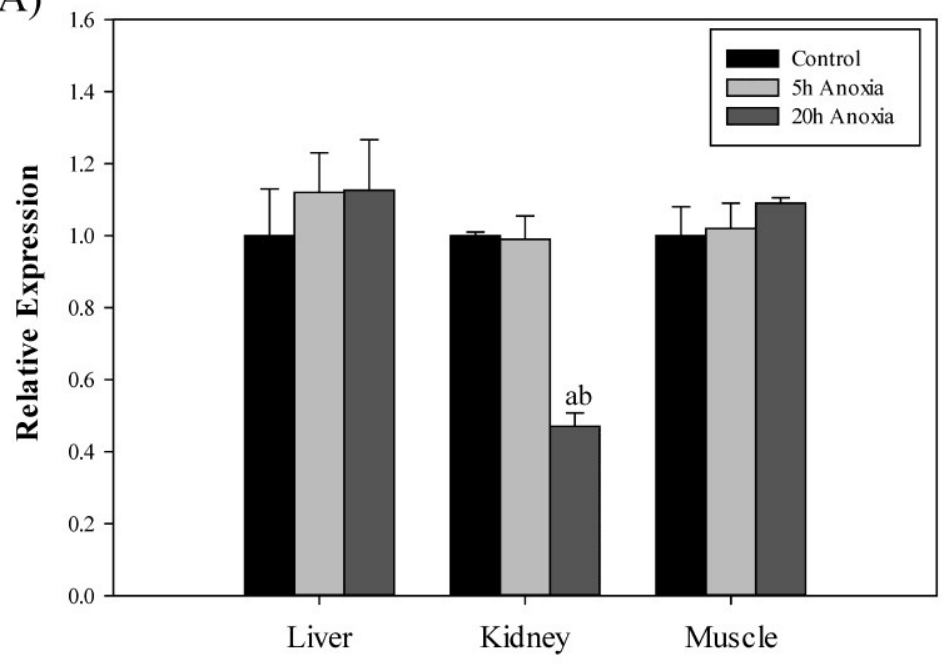

B)

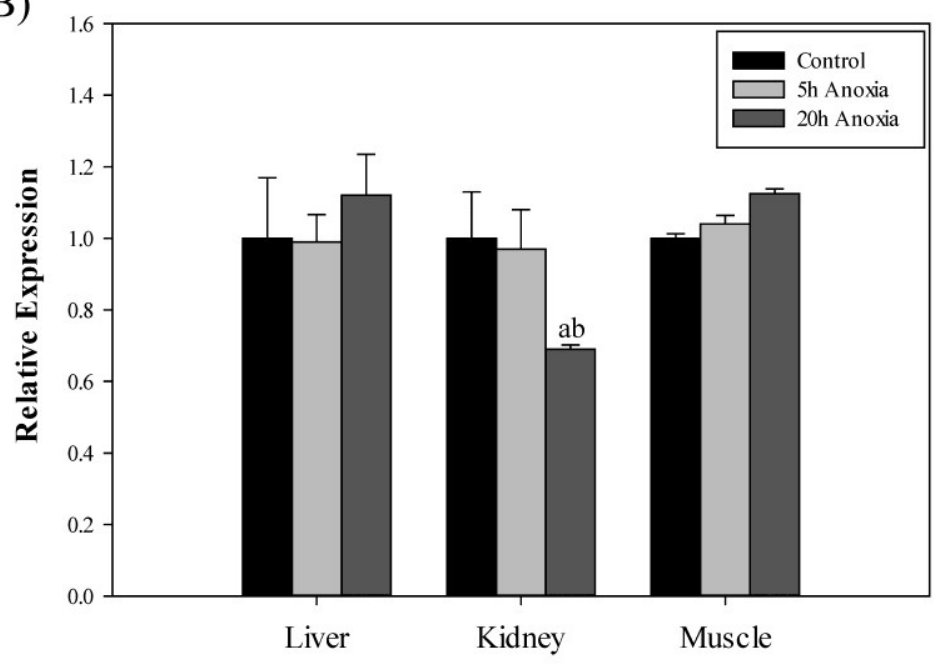

Figure 4.15. Effect of 5 and $20 \mathrm{~h}$ of anoxic submergence on expression levels of the downstream E2F target, PCNA, in T. s. elegans. Histograms show normalized expression of (A) transcript and (B) protein levels under control and anoxic conditions in liver, kidney and white skeletal muscle tissues. Data are means \pm S.E.M., $\mathrm{n}=3-4$ independent trials. a: Significantly different from the corresponding control $(\mathrm{P}<$ $0.05)$; b: significantly different from the $5 \mathrm{~h}$ anoxic value $(\mathrm{P}<0.05)$. 


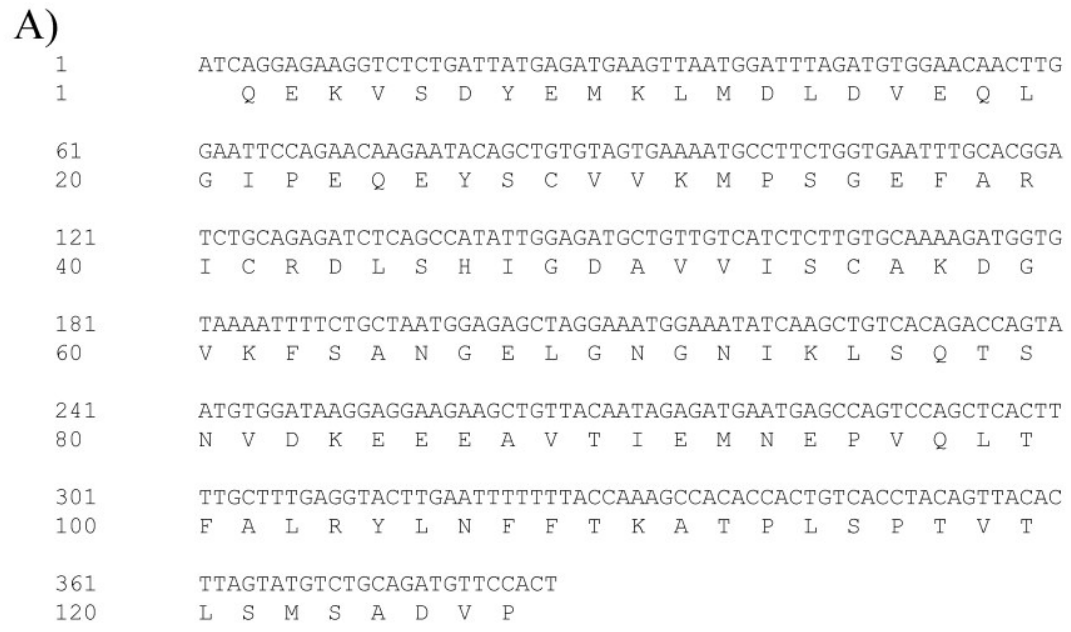

B)
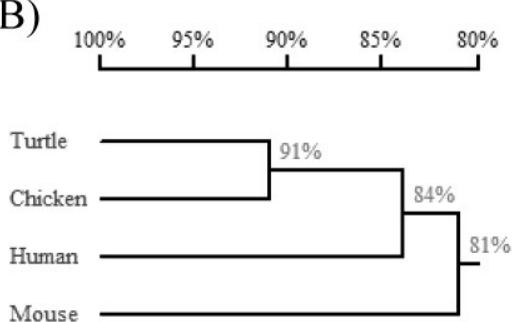

C)
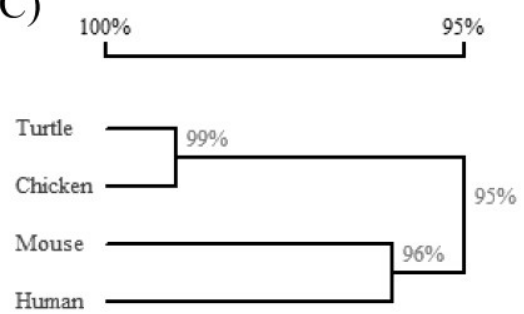

Figure 4.16. Nucleotide and deduced amino acid sequence for T. s. elegans partial pcna sequence. Nucleotides and amino acids are numbered on the left. (A) The nucleotide sequence was 385 nucleotides in length, while the amino acid sequence was 127 amino acids in length. Both the (B) nucleotide and $(\mathrm{C})$ translated peptide sequences were compared to human (H. sapiens), mouse ( $M$. musculus) and, chicken (G. gallus) for homology. 

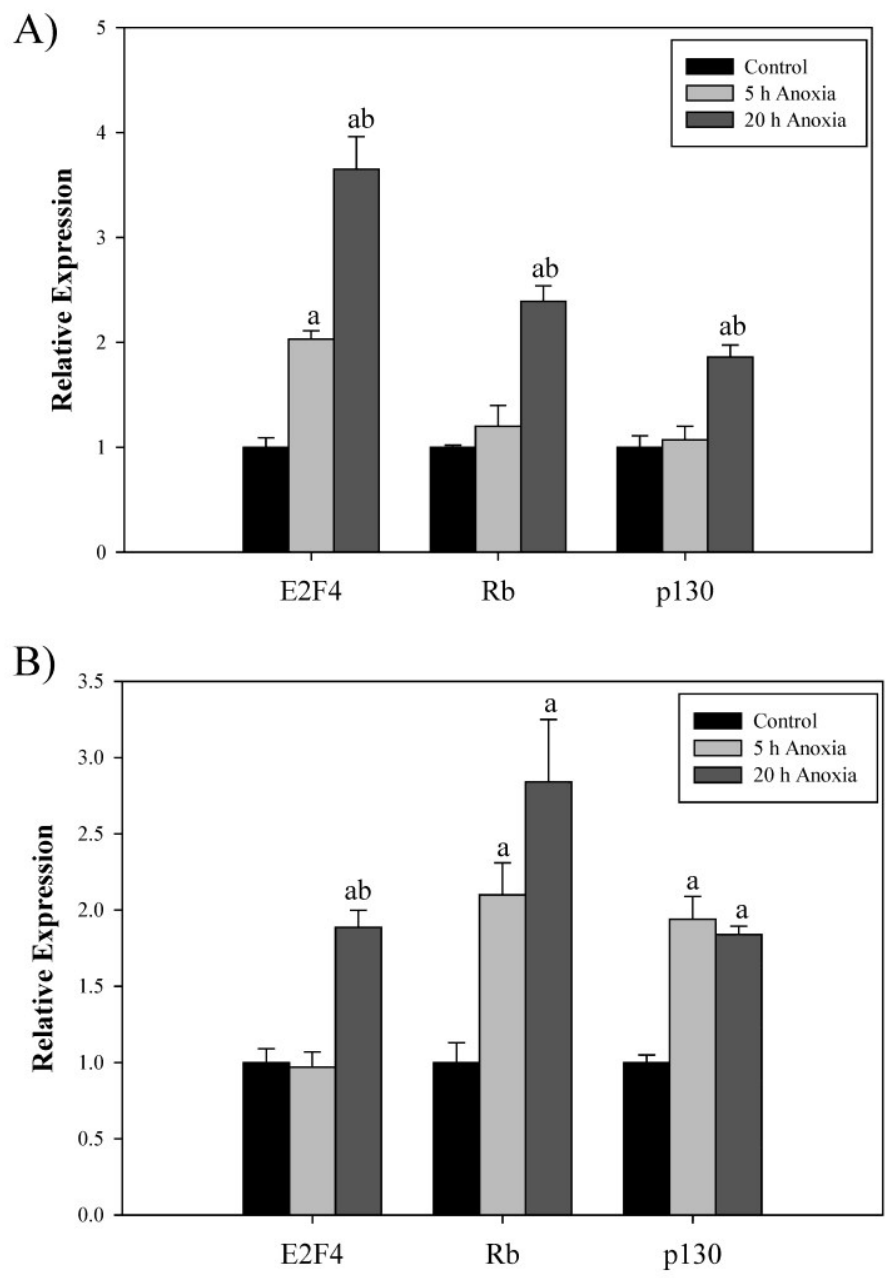

C)

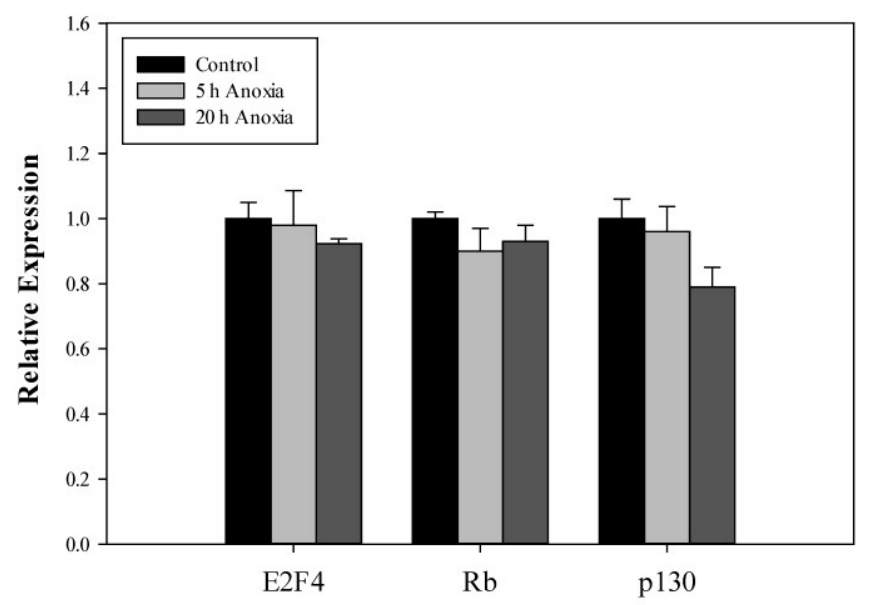

Figure 4.17. Effect of 5 and $20 \mathrm{~h}$ of anoxic submergence on transcript levels of $e 2 f 4, r b$ and $p 130$ in $T . s$. elegans. Histograms show normalized expression levels under control and anoxic conditions in (A) liver, (B) kidney and (C) white skeletal muscle tissues. Data are means \pm S.E.M., $\mathrm{n}=3-4$ independent trials. a: Significantly different from the corresponding control $(\mathrm{P}<0.05)$; b: significantly different from the $5 \mathrm{~h}$ anoxic value $(\mathrm{P}<0.05)$. 
A)

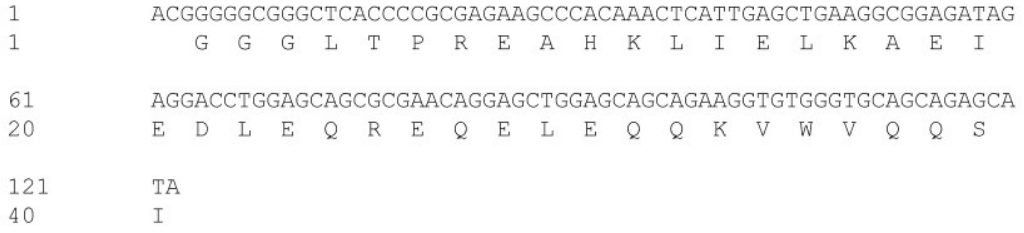

B)
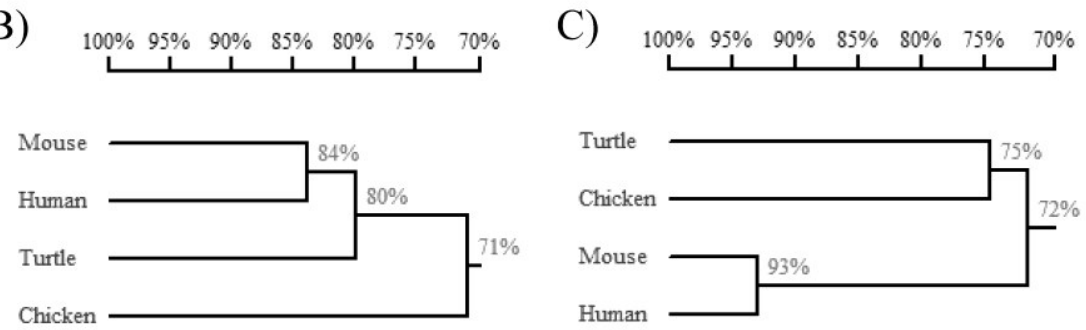

Figure 4.18. Nucleotide and deduced amino acid sequence for $T$. s. elegans partial $e 2 f 4$ sequence. Nucleotides and amino acids are numbered on the left. (A) The nucleotide sequence was 122 nucleotides in length, while the amino acid sequence was 40 amino acids in length. Both the (B) nucleotide and (C) translated peptide sequences were compared to human (H. sapiens), mouse (M. musculus) and, chicken (G. gallus) for homology. 
A)

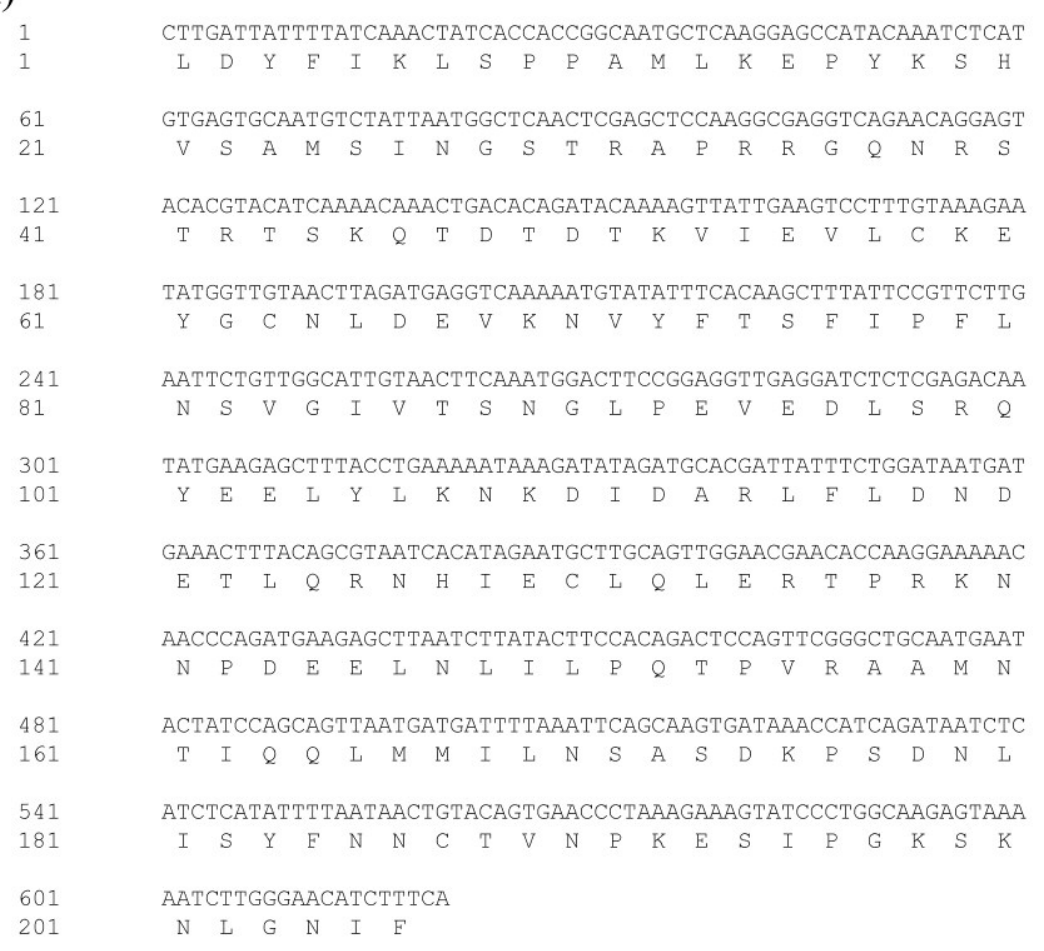

B)
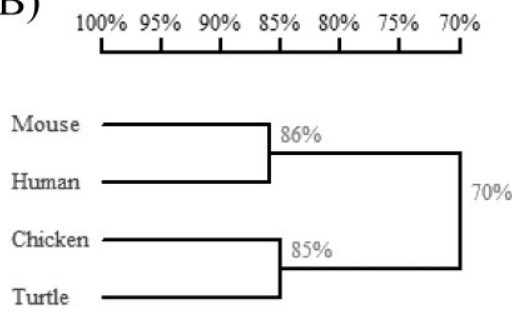
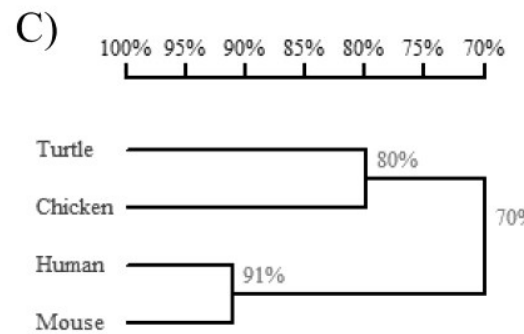

Figure 4.19. Nucleotide and deduced amino acid sequence for $T$. $s$. elegans partial $r b$ sequence. Nucleotides and amino acids are numbered on the left. (A) The nucleotide sequence was 620 nucleotides in length, while the amino acid sequence was 206 amino acids in length. Both the (B) nucleotide and $(\mathrm{C})$ translated peptide sequences were compared to human (H. sapiens), mouse ( $M$. musculus) and, chicken (G. gallus) for homology. 
A)

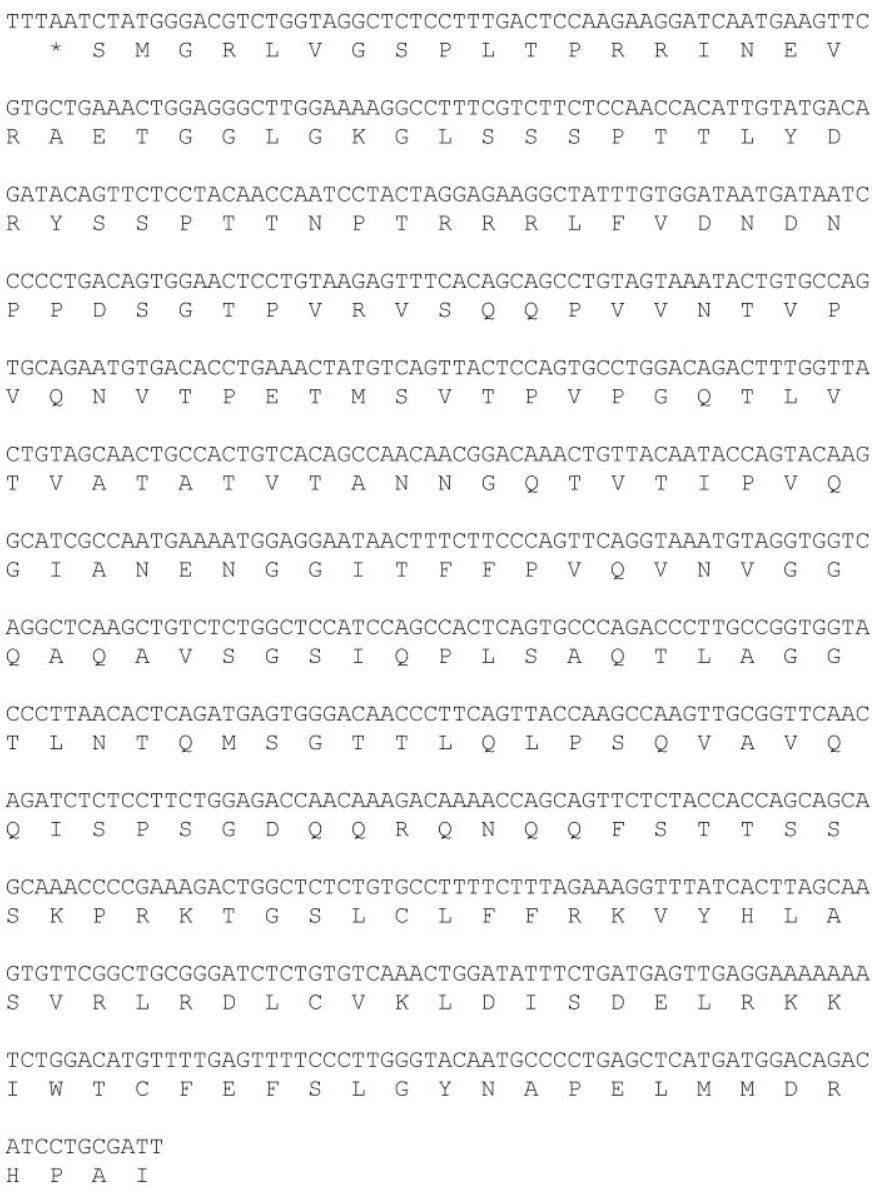

B)

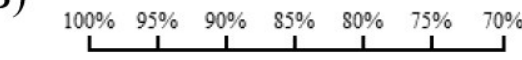

C)
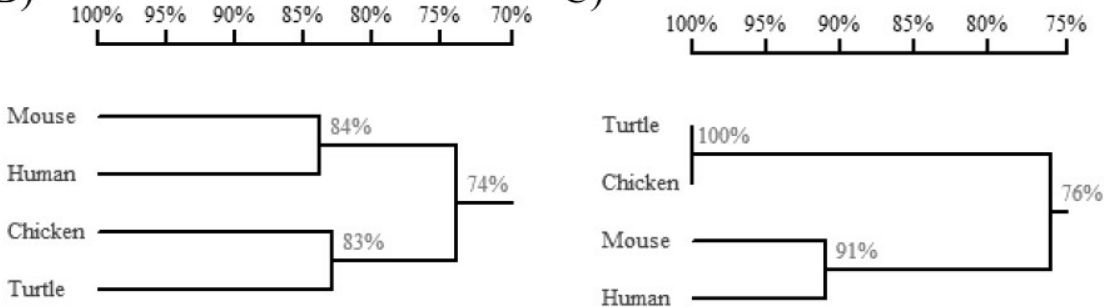

Figure 4.20. Nucleotide and deduced amino acid sequence for T. s. elegans partial pl30 sequence. Nucleotides and amino acids are numbered on the left. (A) The nucleotide sequence was 792 nucleotides in length, while the amino acid sequence was 263 amino acids in length. Both the (B) nucleotide and $(\mathrm{C})$ translated peptide sequences were compared to human (H. sapiens), mouse ( $M$. musculus) and, chicken (G. gallus) for homology. 


\section{CHAPTER 5}

\section{EVIDENCE OF MICRORNA REGULATION OF CELL CYCLE PROTEINS}

Selected data from this chapter have been published:

1) Biggar, K.K. and Storey, K.B. 2012. Cell Cycle, 11, 1705-1713.

2) Zhang, J., Biggar, K.K., and Storey, K.B. 2013. Gene, 513(1), 147-155.

Developed techniques from this chapter have been published: Biggar KK, Kornfeld SF, Storey KB. 2011. Anal. Biochem., 416, 231-233. 


\subsection{Introduction}

It has been long established that the molecular mechanisms of anoxia-induced metabolic rate depression in turtles and other organisms include a global suppression of energy-utilizing processes, balancing the rate of ATP-utilization with the low rates of ATP production from anaerobic glycolysis (Brooks and Storey, 1989; Storey, 2007). Major depressed cellular processes include protein synthesis, gene transcription, and ATP-dependent ion pumps (Hochachka et al., 1997). Despite global reductions in the majority of cellular functions, several cellular pathways that preserve and stabilize cellular macromolecules (such as antioxidant defense and chaperone proteins) are enhanced (Willmore and Storey, 1997; Krivoruchko and Storey, 2010). However, the entrance into a metabolically depressed state does not appear to involve extensive changes to genes expression, undoubtedly because an energy-limited anoxic state is not the time for a major reorganization of the cellular environment. Instead, the types of regulatory mechanisms that are needed for anoxia survival must be broadly applicable to all types of metabolic processes, readily coordinated by extracellular stimuli, and also easily induced and reversed such that few changes to are made protein composition during transitions to and from the stressed state (Storey and Storey, 2004). Interestingly, the characteristics of microRNA regulation match these requirements needed for metabolic depression.

\subsubsection{MicroRNA biogenesis and function}

It has been well established that in response to external stress stimuli, cellular modifications involve transcriptional, translational, post-translational, and allosteric 
regulation (Krutzfeldt and Stoffel, 2006; Safdar et al., 2009). In recent years, however, a new level of rapid and reversible transcriptome regulation, via the action of a special class of microRNA molecules, has emerged into pathway regulation. MicroRNAs (miRNAs) are short, non-coding RNAs capable of regulating protein expression. These 18-25 nucleotide transcripts are able to bind with full or partial complementarity to mRNA targets, resulting in either translational inhibition or degradation of that target (Figures 5.1 and 5.2) (Leung and Sharp, 2010; Grimson et al., 2007; Schier and Giraldez, 2006). A single miRNA may target multiple mRNAs, and a single mRNA may have multiple miRNA binding sites (Lewis et al., 2003; Bartel, 2004). The degree and extent of microRNA binding creates a complex regulatory system that has been predicted to be involved in almost every aspect of biological function. To this point, microRNAs have been shown to be critically involved in biological development, cell differentiation, apoptosis and cell-cycle control (Lewis et al., 2003; Shi and Jin, 2010; Chan et al., 2005; Ivey and Srivastava, 2010). Based on their importance in biological systems in general and their established involvement in models of metabolic rate depression (including freeze tolerance in wood frogs and periwinkle snails, as well as hibernation in squirrels and bats) (Biggar et al., 2009; Morin et al., 2008; Biggar and Storey, 2011; Biggar et al., 2012)., miRNAs are likely to contribute to anoxia tolerance in T. scripta elegans

\subsubsection{Cell cycle regulation}

Given the high cost of cell division and the importance of suppressing nonessential cellular processes under energy-restricted anoxic conditions, it would make sense that cellular proliferation rates are suppressed to a minimum under anoxic 
conditions. Previous reports have suggested that a withdrawal of ATP supply will induce proliferation arrest in the $G_{1}$ phase of the cell cycle (Mazia, 1962). Indeed, research on anoxia tolerance in wood frogs, Rana sylvatica, agrees with these findings. A recent study demonstrated a significant decrease in critical cell cycle components (type D1 and E1 Cyclins, type 2, 4 and 6 Cdks) and an increase in selected CKIs (p16 and p27) that are specific to $\mathrm{G}_{1} / \mathrm{G}_{0}$ arrest after $24 \mathrm{~h}$ of anoxic exposure in wood frogs (Roufayel et al., 2011; Zhang and Storey, 2012; Wu and Storey, 2012). Additionally, it has been speculated that the mechanisms of $\mathrm{G}_{1} / \mathrm{G}_{0}$ cell cycle arrest used bywood frogs may be widespread in multiple models of metabolic rate depression (Biggar and Storey, 2009). Important to this thesis, similar mechanisms of $\mathrm{G}_{1} / \mathrm{G}_{0}$ cell cycle arrest have been discovered for the anoxia tolerant red-eared slider turtle, T. scripta elegans and presented in previous chapters (see Chapters 2, 3 and 4).

The cell cycle is incredibly complex, involving many different proteins and protein complexes at each phase of the cell cycle (O'Connor, 2008). However, a critical point of cell cycle regulation is the initiation phase $\left(G_{1}\right)$. It is this phase that has been shown to be arrest point for many models of anoxia tolerance and metabolic rate depression (Clegg, 1997; Douglas and Haddad, 2003; Foster et al., 2010). Importantly, the activities of Cyclin/Cdk complexes are dictated primarily by the availability of the associated Cyclins. As such, Cyclins function as allosteric regulators acting to regulate the activity of Cdks at specific points of the cell cycle. While Cdks are regularly expressed throughout the cell cycle, Cyclins are translated and shuttled to the nucleus immediately before they are required (Tarn and Lai, 2011). As mentioned in Chapter 2, Cyclin D1 is specific to the $G_{1}$ phase of the cell cycle, associating only with either Cdk4 
or Cdk6, and promoting the initiation of the cell cycle (Matsushime et al., 1994). Unlike other Cyclins that are periodically synthesized during each phase of the cell cycle, the expression of Cyclin D1 is strongly dependent on extracellular signals; acting to relay these signals to core cell cycle machinery (Foster et al., 2010; Sherr, 1995). Due to its unique regulation, Cyclin D1 is regarded as a critical component in initiating cellular proliferation and regulating the $\mathrm{G}_{1}$ phase of the cell cycle (Foster et al., 2010; Matsushime et al., 1994). Cyclin D1 expression and accumulation is influenced by a combination of both transcriptional and translational rates, in addition to protein stability (Tarn and Lai, 2011; Rosenwald et al., 1993).

\subsubsection{Regulation of the cell cycle through Cyclin D1 regulation}

Many studies have reported differential regulation of Cyclin D1 and its effects on the proliferative state of diseased cells (Jiang et al., 1993; Courial et al., 1996; Hayakawa et al., 2011). It has also been reported that mutations occurring in the 3 ' UTR of cyclin d1 are responsible for the hyperproliferative state associated with a variety of tumours (Wiestner et al., 2007). For example, cells expressing cyclin d1 lacking the 3 ' UTR showed a significantly higher rate of proliferation (Wiestner et al., 2007; Deshpande et al., 2009). These studies suggested the presence of negative regulatory elements within the 3' UTR of cyclin $d 1$ that contributed to the regulation of proliferation. It has been suggested that AU-rich elements (AREs) located within the 3'UTR are responsible for cyclin 11 destabilization, and that the loss of the 3' UTR and its associated AREs would lead to an overexpression of Cyclin D1 protein and a hyperproliferative phenotype (Lin et al., 2000). Additionally, studies have discovered that microRNAs of the microRNA- 
15/16 family as well as microRNA-34a directly target the 3' UTR of cyclin d 1 mRNA (Deshpande et al., 2009; Liu et al., 2008; Chen et al., 2008). Importantly, these studies showed that the deletion of the cyclin d1 3'UTR ledd to the loss of both AREs and microRNA binding sites can subsequently to an increase in cyclin $d l$ translation and overexpression (Deshpande et al., 2009). Taken together these studies indicate that the 3'UTR of cyclin $d 1$ is critical to Cyclin D1 regulation and, as a result, may be important in regulating cellular proliferation during periods of cellular stress.

Interestingly, microRNAs that are targeted by p 53 have been shown to increase in expression levels during cell cycle arrest (Tarasov et al., 2007). Additionally, previous research in $T$. scripta elegans has indicated that the transcriptional activity of p53 increased in response to anoxia in both liver and white skeletal muscle tissue, increasing the expression of downstream genes and microRNAs (Zhang et al., 2013). Interestingly, downstream targets of $\mathrm{p} 53$ include $m i R-16-1, m i R-15 a$ and $m i R-34 a$, among other microRNAs (Zhang et al., 2013; Liu et al., 2013; Cui et al., 2013; Tarasov et al., 2007; Chang et al., 2007). Importantly, all three of these p53-regulated microRNAs have been previously shown to regulate cell cycle arrest (Figure 5.3). One study exploring the role of $m i R-34 a$ in Human pancreatic cancer found that this microRNA is suppressed during tumor growth, and that its restoration by transfection was able to reduce tumor growth by 87\% (Ji et al., 2009). When cells were transfected to express high levels of miR-16-1, an increased number of cells were found to be in quiescence with corresponding decreases in the numbers of cells in $S, G_{2}$ and $M$ phases (Linsley et al., 2007). Another study showed that treatment of colon cancer cells with $\mathrm{Cl}$-amidine caused a $\mathrm{G}_{1}$ cell cycle arrest that was mediated by an up-regulation of $m i R-16-1$; and that the expression of this 
microRNA only occurred in the presence of p53 (Cui et al., 2013). Finally, another study showed that $m i R-15 a$ and $m i R-16-1$ are frequently deleted or down-regulated in nonsmall cell lung cancer, and their expression is inversely correlated with the expression of Cyclin D1 (Bandi et al., 2009). Results from these experiments indicated that increased expression of any one of these three microRNA has the ability to arrest cells, a function that is likely mediated by their inhibitory regulation of Cyclin D1 translation.

To examine the post-translational regulation of Cyclin D1 in response to natural anoxic excursions in the red-eared slider turtle, this study examines both the presence of AREs and the role of $t s c-m i R-16-1, t s c-m i R-15 a, t s c-m i R-34 a$ in regulating $c y c l i n d 1$ via its 3'UTR. This analysis yields a focused picture of the regulation of Cyclin D1, its regulation in anoxia tolerance and its adaptation during metabolic rate depression in turtle liver, kidney and white skeletal muscle. 


\subsection{Material and Methods}

\subsubsection{Animal Care and Treatments}

All animal experiments were conducted as described in Chapter 2.

\subsubsection{Total protein isolation}

All protein isolations were conducted as described in Chapter 2.

\subsubsection{Immunoblotting}

Preparation of tissue extracts and western blotting were performed as described in Chapter 2. For immunoblotting, aliquots containing 30-40 $\mu \mathrm{g}$ protein from control and stress conditions were loaded into lanes of either $8 \%$ (Dicer) or 10\% (Cyclin D1) polyacrylamide gels. Gels were run in a Mini Protean III apparatus (BioRad) at $180 \mathrm{~V}$ for $45 \mathrm{~min}$ at $\mathrm{RT}$ ( $60 \mathrm{~min}$ at $4^{\circ} \mathrm{C}$ for Dicer). The resolved proteins were transferred onto nitrocellulose membranes at $70 \mathrm{~V}$ for $1.5 \mathrm{~h}$ at $4{ }^{\circ} \mathrm{C}\left(2 \mathrm{~h}\right.$ at $4{ }^{\circ} \mathrm{C}$ for Dicer $)$. The membranes were then blocked using $1 \mathrm{mg} / \mathrm{ml}$ PVA (70-100 kDa) in TBST for 30-45 sec. After blocking, the membranes were probed with an antigen-specific antibody (see Appendix C). Experimental conditions that are specific to each antibody are listed in Appendix D. Immunoblots were developed using enhanced chemiluminescence reagents.

\subsubsection{Total RNA isolation}

RNA extraction was performed as described in Chapter 2. 


\subsection{5 cDNA synthesis}

Complementary DNA (cDNA) synthesis for cyclin $d 1$ and $\alpha$-tubulin analysis was performed as described in Chapter 2. MicroRNA specific reverse transcription was performed as previously described (Biggar et al., 2011; also see Appendix K for information regarding method development). A $5.0 \mu \mathrm{L}$ aliquot of total RNA $(1 \mu \mathrm{g} / \mu \mathrm{L})$ was incubated with $1.0 \mu \mathrm{L}$ of $250 \mathrm{nM}$ microRNA-specific stem-loop primer (See Appendix H). The reaction was heated at $95^{\circ} \mathrm{C}$ for $5 \mathrm{~min}$ and then incubated for $5 \mathrm{~min}$ at $60^{\circ} \mathrm{C}$. After cooling on ice for $1 \mathrm{~min}$, the remaining reagents $(4 \mu \mathrm{L}$ of $5 \mathrm{X}$ first strand buffer, $2 \mu \mathrm{L}$ of $0.1 \mathrm{M}$ DTT, $1 \mu \mathrm{L}$ of $25 \mathrm{mM}$ dNTPs and $1 \mu \mathrm{L}$ of M-MLV reverse transcriptase) were added. The reaction proceeded for $30 \mathrm{~min}$ at $16^{\circ} \mathrm{C}$, followed by 30 min at $42{ }^{\circ} \mathrm{C}$ and $85{ }^{\circ} \mathrm{C}$ for $5 \mathrm{~min}$. Following reverse transcription, the product was serial diluted to $10^{-3}$ and stored at $-20{ }^{\circ} \mathrm{C}$.

\subsubsection{Polymerase chain reaction}

PCR amplification for cyclin $d 1$ and $\alpha$-tubulin were performed as described in Chapter 2 and the primers and annealing temperature used are listed in Appendices F and G. MicroRNA-specific RT-PCR was performed with $13.25 \mu \mathrm{L}$ of sterile water, $5 \mu \mathrm{L}$ of RT product (experimentally determined dilution), $2 \mu \mathrm{L}$ of $25 \mu \mathrm{M}$ primer mixture (see Appendix H), $2.5 \mu \mathrm{L}$ of $10 X$ PCR buffer, $1.25 \mu \mathrm{L}$ of $50 \mathrm{mM} \mathrm{MgCl}_{2}, 0.5 \mu \mathrm{L}$ of $25 \mathrm{mM}$ dNTPs and $1 \mu \mathrm{L}$ of Taq polymerase. The reactions were amplified for $10 \mathrm{~s}$ at $95{ }^{\circ} \mathrm{C}$, followed by $30-35$ cycles of $95^{\circ} \mathrm{C}$ for $15 \mathrm{~s}$ and $60{ }^{\circ} \mathrm{C}$ for $1 \mathrm{~min}$. PCR products were held at $4{ }^{\circ} \mathrm{C}$ after amplification until quantification. 


\subsubsection{Rapid Amplification of cDNA Ends for the 3' UTR of cyclin $d 1$}

In order to determine whether cyclin $d 1$ mRNA translation in the turtle is regulated in the same manner as in other vertebrates, RACE was used to obtain the 3' UTR. To amplify the 3' end of cyclin $d 1,4 \mu \mathrm{g}$ aliquot of RNA was diluted with DEPC water to a $10 \mu \mathrm{L}$ final volume and used to synthesize cDNA. A $1 \mu \mathrm{L}$ aliquot of $10 \mu \mathrm{M}$ AP primer (5'-GGCCACGCGTCGACTAGTACTTTTTTTTTTTTTTTTT-3') was added to the RNA sample and incubated at $65^{\circ} \mathrm{C}$ for $5 \mathrm{~min}$. The mixture was then chilled rapidly on ice and $4 \mu \mathrm{L} 5 \mathrm{X}$ first strand buffer, $2 \mu \mathrm{L} 100 \mathrm{mM}$ DTT, $1 \mu \mathrm{L}$ of $25 \mathrm{mM}$ dNTPs and $1 \mu \mathrm{L}$ M-MLV reverse transcriptase enzyme were added for a total volume $19 \mu \mathrm{L}$. The mix was incubated at $42{ }^{\circ} \mathrm{C}$ for $1 \mathrm{~h}$ and the reaction was terminated by incubating at 70 ${ }^{\circ} \mathrm{C}$ for $15 \mathrm{~min}$ and chilling to $4{ }^{\circ} \mathrm{C}$. The resulting cDNA was diluted $10^{-2}$ and then amplified using PCR. The PCR reaction of $25 \mu \mathrm{L}$ final volume was composed by mixing $12.5 \mu \mathrm{L}$ of sterile water, $5 \mu \mathrm{L}$ of diluted cDNA, $1 \mu \mathrm{L}$ of $10 \mu \mathrm{M}$ cyclin $d 1$ RACE primer (Appendix F), $1 \mu \mathrm{L}$ of $10 \mu \mathrm{M}$ AP primer, $2.5 \mu \mathrm{L}$ of $10 \mathrm{X}$ PCR buffer, $1.5 \mu \mathrm{L}$ of $50 \mathrm{mM}$ $\mathrm{MgCl}_{2}, 0.5 \mu \mathrm{L}$ of $25 \mathrm{mM}$ dNTPs and $1 \mu \mathrm{L}$ of Taq Polymerase. The PCR reactions were amplified for as follows: $7 \mathrm{~min}$ at $94{ }^{\circ} \mathrm{C}$ followed by 38 cycles of $1 \mathrm{~min}$ at $94{ }^{\circ} \mathrm{C}, 1 \mathrm{~min}$ at $68^{\circ} \mathrm{C}$, and $1.5 \mathrm{~min}$ at $72{ }^{\circ} \mathrm{C}$. The product was $\sim 1650 \mathrm{bp}$ in size.

\subsubsection{MicroRNA-15/16/34a and cyclin d1 Binding Energy}

Theoretical predictions of both the conservation of microRNA seed-pairing sites and the thermodynamics of RNA duplex formation were used to determine the ability of tsc-miR-16-1, tsc-miR-15a and tsc-miR-34a to bind the 3' UTR of cyclin d1. The conservation of microRNA seed-pairing was determined using TargetScan v.5.2. The 
TargetScan program searches for microRNA binding sites (seed matches) conserved between several model organisms (Lewis et al., 2005). Thermodynamic analyses were predicted by using RNAhybrid software and a minimum free energy threshold of less than $-18.0 \mathrm{kcal} / \mathrm{mol}$ (Rehmsmeier et al., 2004; Watanabe et al., 2006; Singh and Nagaraju, 2008).

\subsubsection{Poly-adenylation levels of RNA}

Total RNA extraction was performed as described in Chapter 2. Total RNA samples were normalized in DEPC-treated $\mathrm{ddH}_{2} \mathrm{O}$ and diluted to $0.1 \mu \mathrm{g} / \mu 1$. To detect relative abundance of poly-adenylated RNA, a dot-blot apparatus was used as outlined in the manufacturer's directions (BioRad; Cat\# 170-6545). Biodyne B nylon membrane (Pall Corporation; Cat\#60120) was incubated in $\mathrm{ddH}_{2} \mathrm{O}$ for $15 \mathrm{~min}$ and assembled into the dot-blot apparatus. Wells were washed once with $200 \mu \mathrm{l}$ of $\mathrm{ddH}_{2} \mathrm{O}$ and a vacuum was applied to draw the liquid through the membrane over a period of 5-10 s. Either $1 \mu \mathrm{g}$ or 2 $\mu \mathrm{g}$ of total RNA (diluted to a volume of $200 \mu \mathrm{l}$ in $\mathrm{ddH}_{2} \mathrm{O}$ ) was applied to each well and a vacuum was applied until samples had completely passed through the membrane. Following this, the apparatus was dissembled and the RNA was crosslinked to the membrane by heat after a $2 \mathrm{~h}$ incubation at $80{ }^{\circ} \mathrm{C}$. The membrane was then incubated for $1 \mathrm{~h}$ at $37^{\circ} \mathrm{C}$ in prehybridization buffer (6X SSC, $5 \mathrm{X}$ Denhardt's solution, $0.05 \%$ sodium pyrophosphate, $0.5 \% \mathrm{SDS}, 100 \mu \mathrm{g} / \mathrm{mL}$ salmon sperm DNA [BioShop; Cat\# DNA004.1]). Following incubation, prehybridization buffer was removed and the membrane was incubated in hybridization buffer (6X SSC, 1X Denhardt's solution, 0.05\% sodium pyrophosphate and $50 \mathrm{ng} / \mathrm{mL}$ biotinylated oligo(dT) probe) overnight at room 
temperature (RT). Oligo(dT) probes were purchased with the following sequence (5'biotin-TTTTTTTTTTTTTTTTTTTTTTV-3') from Sigma Aldrich. After hybridization, membranes were washed four times with wash buffer 1 (6X SSC and 0.05\% sodium pyrophosphate) for 5 min each. Membranes were then blocked for $3 \mathrm{~h}$ at RT with blocking buffer (1X TBS, 3\% non-fat skim milk and 0.1\% SDS). After incubation in blocking buffer, a 1:1000 dilution of streptavidin-HRP conjugate was added to the blocking buffer and allowed to incubate for another $1 \mathrm{~h}$. Membranes were then washed three times with wash buffer 2 (1X TBS and $0.1 \%$ SDS) for 10 min each and then developed using enhanced chemiluminescence. The GeneTools program was used to quantify the RNA dots (Syngene, Frederick, MD). RNA dots where normalized by the relative 18s rRNA levels from the same total RNA sample.

\subsubsection{Statistics}

Detection of bands on gels and blots used the ChemiGenius Bio-Imaging System (Syngene, Frederick, MD) and densitometric analysis was performed with the associated Gene Tools software. Immunoblotting with $\alpha$-tubulin-specific antibodies showed constant $\alpha$-tubulin expression under all experimental conditions and when compared with the combined density of a group of Coomassie stained protein bands. Immunoblots of the proteins of interest were individually adjusted for loading irregularities by normalizing the band intensity of immune-reactive material in each lane against the combined density of a group of Coomassie-stained protein bands in the respective sample lane. These Coomassie-stained proteins showed similar expression pattern to $\alpha$-tubulin and the same stained bands were used for each such comparison. For RT-PCR, band intensity was 
normalized against the corresponding intensity of $\alpha$-tubulin (for cyclin $d 1$ ) or $5 S$ rRNA (for tse-miR-16-1, tse-mRNA-15a and tse-miR-34a) bands amplified from the same RNA sample. Mean normalized band densities \pm SEM were calculated for control and anoxic samples and significance testing used a one-way analysis of variance followed by the Student-Newman-Keuls test for the control versus 5 and $20 \mathrm{~h}$ anoxia data. Statistical difference was accepted if $P<0.05$; all data are derived from multiple independent tissue extracts from different animals. 


\subsection{Results}

\subsubsection{Cyclin D1 Expression (results from Chapter 2)}

Cyclin D1 protein decreased significantly under both 5 and $20 \mathrm{~h}$ anoxia to $59 \pm 1 \%$ and $61 \pm 9 \%$ of control values in liver tissue, respectively $(P<0.05)$. Similar decreases in total protein expression of Cyclin D1 were detected in kidney, decreasing during 5 and 20 h anoxia to $89 \pm 1$ and $66 \pm 8 \%$ of control values, respectively $(P<0.05)$. No significant changes in Cyclin D1 expression were detected in white skeletal muscle. Several studies have reported that the state of Cyclin D1 degradation is dependent on the initial phosphorylation of amino acid Thr286, followed by poly-ubiquitination and subsequent proteasomal degradation. To assess the state of Cyclin D1 degradation, the ratio of phosphorylated Cyclin D1 (Thr286) expression to total cellular protein levels of Cyclin D1 was calculated in control vs. anoxic tissues (Figure 5.4). Antibodies used to assess the phosphorylated state of p-Cyclin D1 (Thr286) detect endogenous expression of Cyclin D1 only when phosphorylated at threonine 286. The ratio of phosphorylated Cyclin D1 (Thr286) to total cellular levels of Cyclin D1 protein did not significantly change between control and anoxic states in all three tissues examined (Figure 5.4B). The expression of cyclin 11 mRNA was analyzed using RT-PCR. Unlike the stress response seen in total Cyclin D1 protein expression in liver and kidney, no significant changes in cyclin $d 1$ transcript levels were detected in any of the three tissues examined (Figure $5.4 \mathrm{C})$. 


\subsubsection{Identification of regulatory elements within the cyclin D1 3' UTR}

The 3'UTR sequence of cyclin $d 1$ mRNA from $T$. scripta elegans was obtained using 3' RACE and analyzed for elements that could potentially regulate Cyclin D1 translation. The 3'UTR contained a total of 7 AU elements with a cluster of four reiterations of AU elements within a 273 base region (Figure 5.5). Analysis of putative microRNA binding sites was performed using both TargetScan 5.2 and RNAhybrid. Results identified that the 3'UTR of turtle cyclin $d 1$ contained conserved binding sequences for $t s c-m i R-16-1, t s c-m i R-15 a$ and $t s c-m i R-34 a$ (Figure 5.6). Importantly, two of the conserved binding sites were shared by miR-16-1 and miR-15a. All of these microRNA binding sites were conserved between multiple vertebrate species including, but not limited to: Gallus gallus, Homo sapiens, Pan troglodytes, Rattus norvegicus, Mus musculus and Canis familiaris.

\subsubsection{Minimum free energy of cyclin $D 1 / m i R N A$ binding}

To determine the likelihood of cyclin 11 :microRNA binding, the theoretical minimum free energy (mfe) of binding ( $\mathrm{kcal} / \mathrm{mol})$ of RNA duplex formation was analyzed (Figure 5.7). The theoretical mfe was calculated using the program RNAhybrid. All theoretical predictions of RNA duplex formation and thermodynamic binding parameters for $t s c-m i R-16-1$, $t s c-m i R-15 a$ and $t s c-m i R-34 a$ are listed in Table 5.1. All microRNA binding interactions occurred with a favourable mfe less than -18.0 $\mathrm{kcal} / \mathrm{mol}$. 


\subsubsection{Relative expression of Dicer}

Changes in the relative expression of Dicer protein in response to anoxia were assessed using immunoblotting (Figure 5.8). The Dicer antibody used in this study crossreacted with a protein band of $\sim 250 \mathrm{kDa}$. In liver, the total protein expression of Dicer significantly increased by $3.06 \pm 0.37$-fold after $5 \mathrm{~h}$ as compared to control levels $(P<$ 0.05 ) and decreased back to control levels by $20 \mathrm{~h}$ anoxia. The total protein expression of Dicer in kidney did not significantly change in response to either 5 or $20 \mathrm{~h}$ anoxia. In white skeletal muscle, the relative expression of Dicer protein increased by $1.80 \pm 0.15$ and $1.74 \pm 0.05$-fold compared to control levels after 5 and $20 \mathrm{~h}$ anoxia, respectively $(P<$ $0.05)$.

\subsubsection{MicroRNA expression}

Using a microRNA RT-PCR protocol developed specifically for this thesis, the expression of $t s c-m i R-16-1$ (Figure 5.9), tsc-miRNA-15a (Figure 5.10) and tsc-miR-34a (Figure 5.11) were assessed in response to anoxia in liver, kidney and white skeletal muscle tissues of T. scripta elegans. Both tsc-miR-16-1 and tsc-miR-15a displayed an inverse correlation with Cyclin D1 protein levels in both liver and kidney in response to anoxia. Similar to the expression of Cyclin D1 protein, both $t s c-m i R-16-1$ and $t s c-m i R-$ $15 a$ showed no significant change in response to anoxia in white skeletal muscle.

Expression levels of $t s c-m i R-16-1$ increased significantly in liver by $2.58 \pm 0.18$ fold after $5 \mathrm{~h}$ anoxia and 3.11 \pm 0.13 -fold after $20 \mathrm{~h}$ anoxia when compared to control values $(P<0.05)$. Similar trends were seen in kidney with $t s c-m i R-16-1$ significantly increased by $1.36 \pm 0.03$-fold after $5 \mathrm{~h}$ anoxia and $1.54 \pm 0.07$-fold in response to $20 \mathrm{~h}$ 
anoxia when compared to control values $(P<0.05)$. No significant changes in $t s c-m i R-$ 16-1 expression were detected in skeletal muscle in response to anoxic exposure. Similar to $t s c-m i R-16-1$ expression, $t s c-m i R-15 a$ increased significantly in liver tissue by $1.60 \pm 0.04$-fold after $5 \mathrm{~h}$ anoxia and $2.31 \pm 0.15$-fold in response to $20 \mathrm{~h}$ anoxia when compared to control values $(P<0.05)$. Significant changes in $t s c-m i R-15 a$ expression in kidney were only after $20 \mathrm{~h}$ anoxia; levels significantly increased by $1.61 \pm 0.12$-fold in response to $20 \mathrm{~h}$ anoxia when compared to control values $(P<0.05)$. No significant changes in $t s c-m i R-15 a$ expression were seen in white skeletal muscle in response to anoxic exposure. In response to anoxia the relative expression of $t s c-m i R-34 a$ increased significantly in liver tissue by $2.12 \pm 0.20$-fold after $5 \mathrm{~h}$ anoxia and $2.67 \pm 0.20$-fold in response to $20 \mathrm{~h}$ anoxia as compared to controls $(P<0.05)$. In kidney, the relative expression of $t s c-m i R-34 a$ significantly increased by $1.42 \pm 0.06$-fold after $5 \mathrm{~h}$ anoxia and $1.61 \pm 0.12$-fold in response to $20 \mathrm{~h}$ anoxia when compared to control values $(P<0.05)$. Similar to both liver and kidney, in white skeletal muscle the relative expression of $t s c$ $m i R-34 a$ increased by $1.24 \pm 0.01$ and $1.99 \pm 0.03$-fold compared to control values after 5 and $20 \mathrm{~h}$ anoxia, respectively $(P<0.05)$.

\subsubsection{Relative poly-adenylation levels of mRNA}

To examine the relative levels of translatable mRNA, biotin-labeled oligo(dT) probes were used to detect relative poly-adenylated mRNA levels in the anoxic turtle (Figure 5.12). Additionally, a standard curve consisting of $0.5-2 \mu \mathrm{g}$ of total RNA was generated to determine the appropriate amount of RNA to use in the experiment (Figure 5.12C). In all tissues studied (liver, kidney and white skeletal muscle), no significant 
changes in the relative amount of poly-adenylated RNA were detected in response to either 5 or $20 \mathrm{~h}$ of anoxia exposure. 


\subsection{Discussion}

Previous research examining the individual proteins that regulate cell proliferation identified Cyclin D1 as a crucial component in initiating the cell cycle (Foster et al., 2010). As such, the protein expression of Cyclin D1 is tightly coordinated with each cell cycle phase and is translated by the cell immediately before it is required. Many studies have reported that an increase in Cyclin D1 protein is indicative of an increased proliferative state, and the entrance of a cell into the $\mathrm{G}_{1}$ phase of the cell cycle (Stacey, 2003; Masamha and Benbrook, 2009). Appropriately, several studies have also indicated that a reduction of cyclin D1 protein may be a marker of reduced proliferation and cell cycle arrest (Masamha and Benbrook, 2009; Nishi et al., 2009). The concept of cell cycle arrest has been previously hypothesized to be an important part of the overall hypometabolic response of the anoxic turtle (T. scripta elegans) and was predicted to include strict regulation of Cyclin D1 protein expression as well as several inhibitors of the cell cycle (p16 and p27) (Biggar and Storey, 2009). The findings of this study tend to agree with this hypothesis.

The responses of Cyclin D1 protein to anoxia were similar in proliferative tissues, kidney and liver, of T. scripta elegans (a decrease to about $60 \%$ of control values) and Cyclin D1 levels were unchanged in a primarily senescent tissue (white skeletal muscle). This result for skeletal muscle and may be a reflection of the tissue's general post-mitotic state; perhaps non-reversible mechanisms of cell cycle arrest, such as that imposed by heterochromatin formation, are permanently utilized independent of oxidative stress (Zhang and Adams, 2007). Interestingly, the expression of cyclin d1 mRNA did not 
correlate with protein expression and did not to change with anoxic exposure in any of the examined tissues.

Differential expression of mRNA and protein is not uncommon and is typically thought to be a result of either protein degradation or post-transcriptional regulation of mRNA (either through translational silencing or mRNA degradation). Indeed, proteasomal degradation of Cyclin D1 could contribute substantially to the overall reduction in protein expression seen in the anoxic turtle. Studies have reported that the degradation of Cyclin D1 is dependent on an initial phosphorylation of threonine 268 by GSK-3 $\beta$ (Masamha and Benbrook, 2009; Alao, 2007). Phosphorylation of this amino acid is necessary for poly-ubiquitination and subsequent proteasomal degradation. However, the relative amount of p-Cyclin D1 (Thr268), compared to total Cyclin D1 protein did not change significantly in any of the tissues examined. Given that mRNA levels remain unchanged during anoxia, this may suggest that the decrease of Cyclin D1 protein is a result of decreased translation and not an increase in protein degradation.

It has been proposed that the 3'UTR of cyclin $d 1$ is critical to regulating Cyclin D1 translation due to a combination of several regulatory elements, including AREs and microRNA binding sites (Wiestner et al., 2007; Rimokh et al., 1994). Recent studies examining the expression of cyclin d1, with and without the 3' UTR, have demonstrated that deletion of the 3' UTR (and the regulatory sites located within) in human fibroblasts leads to a hyper-proliferative state (Deshpande et al., 2009). This same study further demonstrated that Cyclin D1 translation is dependent on the presence of both an AU-rich region and microRNA binding sites. To determine the potential for a similar mode of regulation in T. scripta elegans, the 3' UTR was sequenced and analyzed for the presence 
of AU-rich regions and potential microRNA binding sites (Figures 5.5 and 5.6).

Exploration of the 3' UTR sequence of turtle cyclin d1 showed an AU-rich region containing four AUUUA repeats within a 273 nucleotide region. A number of studies have suggested that the presence of an AU-rich region within the 3' UTR of cyclin d1 leads to an increase in mRNA destabilization and therefore could contribute to a decrease in cyclin dl mRNA and consequently a decrease in Cyclin D1 expression (Lin et al., 2008; Guo et al., 2005). However, results from this turtle study are not suggestive of mRNA destabilization; the expression of cyclin $d 1$ mRNA does not change significantly with anoxia exposure. Indeed, other studies have shown that AU-rich regions in the 3'UTR of cyclin $d l$ do not lead to destabilization of the mRNA, but instead provide a stabilizing effect (Deshpande et al., 2009). This finding agrees with the present turtle research, since the presence of AU-rich regions did not appear to induce mRNA destabilization. Instead, negative regulation of Cyclin D1 translation may be imparted by the presence of conserved microRNA binding sites for tsc-miR-16-1, tsc-miR-15a and tsc-miR-34a.

To explore this further, the expression profiles of the three microRNAs were determined and shown to be inversely correlated with the expression of Cyclin D1 protein in liver and kidney. This correlation may be indicative of microRNA-induced translational suppression of cyclin $d 1$, where an increased expression of miR-16-1, miR$15 a$ and/or $m i R-34 a$ leads to a reduction of mRNA translation in proliferative tissues. In skeletal muscle, however, no significant changes occurred in either Cyclin D1 protein or $m i R-16-1$ and $m i R-15 a$ expression although $m i R$-34a levels increased in response to both 5 and $20 \mathrm{~h}$ anoxia. Although the functions of $m i R-16-1$ and $m i R-15 a$ are mainly restricted 
to regulating the cell cycle, the functions of $m i R-34 a$ also include activation of the transcription factor, NF- $\mathrm{KB}$ (through the inhibition of SirT1 translation) (Tan et al., 2012). Indeed, the response of NF- $\kappa \mathrm{B}$ to anoxia could represent a potentially important molecular event as it is an oxygen-responsive transcription factor that promotes the expression of many target genes, including genes involved in antioxidant defense, cell growth and differentiation and apoptosis (Pahl, 1999). As such, $m i R-34 a$ may function in regulating NF- $\kappa \mathrm{B}$ during periods of anoxia and this would justify the increase seen in white skeletal muscle, independent of cyclin $d 1$ regulation.

MicroRNAs are known to be involved in fine-tuning mRNA expression, regulating both mRNA degradation and translational silencing depending on the degree of binding (Bartel, 2009). Important to microRNA binding and regulation is the presence of both a seed region (nearly perfect binding with nucleotides 2-7 on the 5'end of microRNA) and associated complementary binding (binding with the 3 ' end of microRNA). The degree of binding between the two RNA strands dictates the fate of the target mRNA. RNA duplexes displaying perfect Watson-Crick base pairing are cleaved by the endonuclease activity of the agonaute (Ago) protein, whereas imperfect structures are likely targeted to mRNA storage molecules, including p-bodies and stress granules (Bartel, 2009; Liu et al., 2005; Leung et al., 2006). In addition to the degree of microRNA/target complementarity, the thermodynamics of microRNA/target hybridization are also crucial to the stability of the RNA duplex structure $\left(\Delta \mathrm{G}^{\circ}\right.$ typically less than $-18 \mathrm{kcal} / \mathrm{mol}$ ) (Singh and Nagaraju, 2008). Theoretical analysis of the microRNA binding site within the turtle cyclin $d 1$ 3'UTR, suggested that there are two conserved sites that are able to bind either $m i R-16-1$ or $m i R-15 a$ and one conserved 
binding site for $m i R-34 a$, all with a thermodynamically favourable $\Delta \mathrm{G}^{\circ}$ values of less than $-18 \mathrm{kcal} / \mathrm{mol}$ (Table 5.1). Additionally, the imperfect binding between cyclin $d 1$ and all three microRNAs suggests a mechanism for translational silencing and not mRNA degradation. This discovery provides a potential mechanism to decrease Cyclin D1 protein without decreasing the expression of cyclin $d 1$ mRNA, such as that seen in the hypometabolic turtle.

To further explore the overall regulation of microRNA regulation under anoxic conditions, the protein expression of the major microRNA processing enzyme, Dicer, was also assessed. Interestingly, the relative expression of Dicer protein displayed a tissue specific response to anoxia. In liver and white muscle tissue, Dicer protein significantly increased after $5 \mathrm{~h}$ anoxia but no change was found in kidney. Furthermore, after $20 \mathrm{~h}$ anoxia Dicer expression was maintained in white skeletal muscle, decreased to normoxic levels in liver tissue. Although Dicer protein expression is not an indication of microRNA processing activity, it does suggest that microRNA processing may be relatively more important to anoxic survival in liver and white skeletal muscle. To this point, previous studies in the anoxic turtle have provided evidence for possible post-transcriptional regulation of protein expression during anoxia; a process that could be facilitated by microRNA function (Land et al., 1993; Douglas et al., 1994).

A reduction in the protein synthesis of Cyclin D1 could be achieved in two ways; (1) through the reduction in mRNA template levels and/or (2) through differential regulation of the ribosomal translational machinery. Due to the high cost of transcription ( $10 \%$ of ATP turnover during normoxia) one would expect to see a downregulation of RNA synthesis under anoxia, leading to a reduction in protein synthesis. However, results 
from this chapter show that total translatable RNA (ie. poly-adenylated content) did not change significantly in the anoxic turtle (Figure 5.12). Interestingly, complementary studies have documented no change in total translatable RNA concentrations after $16 \mathrm{~h}$ of anoxia in liver, kidney, heart and white skeletal muscle of T. scripta elegans (Douglas et al., 1994). Additionally, previous studies have shown that the RNA-to-protein ratio does not significantly change in T. scripta elegans liver after $12 \mathrm{~h}$ of anoxia (Land et al., 1993). Hence, the decrease in protein synthesis rates exhibited during anoxia do not appear to be controlled by tissue RNA concentration. Instead, reversible control of the rate of protein synthesis by microRNA could be an integral part of the turtle's hypometabolic response to anoxia.

In summary, this study provides the first demonstration of cyclin $d 1$ regulation in the anoxic turtle, T. scripta elegans and suggests potential implications towards cell cycle regulation by microRNA. Taken together with the thermodynamic likelihood of microRNA binding, this study suggests that $m i R-16-1, m i R-15 a$ and $m i R-34 a$ could have a role in reducing Cyclin D1 protein expression through repression of translation during anoxia. Additionally, these microRNAs may have a large influence on the state of cell proliferation since both $m i R-16-1$ and $m i R-15 a$ have been shown to target multiple components of the cell cycle including E2F5, cdk4, cdk6 and cyclin e1. Given the extensive reach of microRNA targeting, further analysis is required to assess the degree of influence exerted by $m i R-16-1$ and $m i R-15 a$ on multiple components of the cell cycle during periods of hypometabolism. With the enormous regulatory potential and significant molecular crosstalk of microRNA, these small regulatory molecules may have widespread effects in coordinating and implementing the suppression of protein 
synthesis, thereby contributing to global metabolic rate depression. As such, the characteristics of microRNA regulation dovetail with the need for metabolic depression to be broadly applicable, readily coordinated, easily induced and readily reversed. Gene regulation by microRNA target repression and degradation may prove to be a critical component of metabolic rate depression. 


\section{References}

Alao, J.P. 2007. The regulation of cyclin D1 degradation: roles in cancer development and the potential for therapeutic invention. Mol. Cancer, 6, 24-40.

Bandi, N., Zbinden, S., Gugger, M., Arnold, M., Kocher, V., Hasan, L., Kappeler, A., Brunner, T., and Vassella, E. 2009. MiR-15a and miR-16 are implicated in cell cycle regulation in a $\mathrm{Rb}$-dependent manner and are frequently deleted or downregulated in non-small cell lung cancer. Cancer Res., 69, 5553-5559.

Bartel, D.P. 2004. MicroRNAs: genomics, biogenesis, mechanism and function. Cell, 116, 281-297.

Bartel, D.P. 2009. MicroRNAs: Target recognition and regulatory functions. Cell, 136(2), 215-233.

Biggar, K.K., and Storey, K.B. 2009. Perspectives in cell cycle regulation: Lessons from an anoxic vertebrate. Curr. Genom., 10, 573-584.

Biggar, K.K., Dubuc, A., and Storey, K.B. 2009. MicroRNA regulation below zero: Differential expression of miRNA-21 and miRNA-16 during freezing in wood frogs. Cryobiology, 59(3), 317-321.

Biggar, K.K., and Storey, K.B. 2011. The emerging roles of microRNAs in the molecular responses of metabolic rate depression. J. Mol. Cell Biol., 3, 167-175.

Biggar, K.K., Kornfeld, S.F., and Storey, K.B. 2011. Amplification and sequencing of mature microRNAs in uncharacterized animal models using stem-loop RT-PCR. Anal. Biochem., 416, 231-233.

Biggar, K.K., Kornfeld, S.F., Maistrovski, Y., and Storey, K.B. 2012. MicroRNA regulation in extreme environments: Differential expression of microRNAs in the intertidal snail Littorina littorea during extended periods of freezing and anoxia. Genom. Proteom. Bioinform., 10(5), 302-309.

Brooks, S.P.J., and Storey, K.B. 1989. Regulation of glycolytic enzymes during anoxia in the turtle Pseudemys scripta. Am. J. Physiol., 257, R278-R283.

Calin, G.A., Dumitru, C.D., Shimizu, M., Bichi, R., Zupo, S., Noch, E.,Aldler, H., Rattan, S., Keating, M., Rai, K., Rassenti, L., Kipps, T., Negrini, M., Bullrich, F., and Croce, C.M. 2002. Frequent deletionsand down-regulation of micro-RNA genes miR15 and miR16 at13q14 in chronic lymphocytic leukemia. Proc. Natl. Acad. Sci. U.S.A., 99, 15524-15529.

Chan, J., Krichevsky, A., and Kosik, K. 2005. MicroRNA-21 is an antiapoptotic factor in human glioblastoma cells. Cancer Res., 65(14), 6029-6033.

Chang, T., Wentzel, E., Kent, O., Ramachandran, K., Mullendore, M., Lee, K., Feldmann, G., Yamakuchi, M., Ferlito, M., Lowenstein, C., Arking, D., Beer, M., Maitra, A. and Mendell, J. 2007. Transactivation of miR-34a by p53 broadly influences gene expression and promotes apoptosis. Mol. Cell., 26, 745-752. 
Chen, R.W., Bemis, L.T., Amato, C.M., Myint, H., Tran, H., Birks, D.K., Eckhardt, S.G., and Robinson, W.A. 2008. Truncation in CCND1 mRNA alters miR-16-1 regulation in mantle cell lymphoma. Blood, 112, 822-829.

Clegg, J.S. 1997. Embryos of Artemia franciscana survive four years of continuous anoxia: the case for complete metabolic rate depression. J. Exp. Biol., 200, 467475.

Courial, F., Louason, G., Speiser, P., Katsaros, D., Zeillinger, R., and Theillet, C. 1996. Cyclin gene amplification and overexpression in breast and ovarian cancers: evidence for the selection of cyclin D1 in breast and cyclin E in ovarian tumors. Int. J. Cancer, 69(4), 247-253.

Cui, X., Witalison, E.E., Chumanevich, A.P., Chumanevich, A.A., Poudyal, D., Subramanian, V., Schetter, A.J., Harris, C.C., Thompson, P.R., and Hofseth, L.J. 2013. The induction of microRNA-16 in colon cancer cells by protein arginine deiminase inhibition causes a p53-dependent cell cycle arrest. PLoS ONE, 8(1), e53791.

Deshpande, A., Pastore, A., Deshpande, A.J., Zimmermann, Y., Hutter, G., Weinkauf, M., Buske, C., Hiddemann, W., and Dreyling, M. 2009. 3' UTR mediated regulation of the cyclin D1 proto-oncogene. Cell Cycle, 8(21), 3584-3592.

Douglas, R., and Haddad, G. 2003. Effect of oxygen deprivation on cell cycle activity: a profile of delay and arrest. J. Appl. Physiol., 94, 2068-2083.

Douglas, D.N., Giband, M., Altosaar, I., and Storey, K.B. 1994. Anoxia induces changes in translatable mRNA populations in turtle organs: a possible adaptive strategy for anaerobiosis. J. Comp. Physiol. B, 164, 405-414.

Foster, D.A., Yellen, P., Xu, L., and Saqcena, M. 2010. Regulation of G1 cell cycle progression: Distinguishing the restriction point from a nutrient-sensing cell growth checkpoint(s). Genes Cancer, 1(1), 1124-1131.

Guo, Y., Harwalkar, J., Stacey, D.W., and Hitomi, M. 2005. Destabilization of cyclin D1 message plays a critical role in cell cycle exit upon mitogen withdrawal. Oncogene, 24, 1032-1042.

Grimson, A., Farh, K., Johnston, W., Garrett-Engele, P., and Bartel, D. 2007. MicroRNA targeting specificity in mammals: determinants beyond seed pairing. Mol. Cell, 27, 91-105.

Hayakawa, Y., Hirata, Y., Nakagawa, H., Sakamoto, K., Hikiba, Y., Kinoshita H., Nakata, W., Takahashi, R., Tateishi, K., Tada, M., Akanuma, M., Yoshida, H., Takeda, K., Ichijo, H., Omata, M., Maeda, S., and Koike, K. 2011. Apoptosis signal-regulating kinase 1 and cyclin D1 compose a positive feedback loop contributing to tumor growth in gastric cancer. Proc. Natl. Acad. Sci. USA, 108(2), 708-785.

Hochachka, P.W., Land, S.C., and Buck, L.T. 1997. Oxygen sensing and signal transduction in metabolic defense against hypoxia: lessons from vertebrate facultative anaerobes. Comp. Biochem. Physiol. A, 118(1), 23-29. 
Ivey, K., and Srivastava, D. 2010. MicroRNAs as regulators of differentiation and cell fate decisions. Cell Stem Cell., 7(1), 36-41.

Jackson, D.C. 1997. Lactate accumulation in the shell of the turtle Chrysemys picta bellii during anoxia at $3{ }^{\circ} \mathrm{C}$ and $10^{\circ}$ C.J. Exp. Biol., 200(7), 2295-2300.

Ji, Q., Hao, X., Zhang, M., Tang, W., Yang, M., Li, L., Xiang, D., DeSano, J.T., Bommer, G.T., Fan, D., Fearon, E.R., Lawrence, T.S., and Xu, L. 2009. MicroRNA miR-34 inhibits human pancreatic cancer tumor-initiating cells. PLoS ONE, 4(8), e6816.

Jiang, W., Kahn, S.M., Zhou, P., Zhang, Y.J., Cacace, A.M., Infante, A.S., Doi, S., Santella, R.M., and Weinstein, I.B. 1993. Overexpression of cyclin D1 in rate fibroblasts causes abnormalities in growth control, cell cycle progression and gene expression. Oncogene, 8(12), 3447-3457.

Krivoruchko, A., and Storey, K.B. 2010. Regulation of the heat shock response under anoxia in the turtle, Trachemys scripta elegans. J. Comp. Physiol. B, 180(3), 403414.

Land, S.C., Buck, L.T., and Hochachka, P. W. 1993. Response of protein synthesis to anoxia and recovery in anoxia-tolerant hepatocytes. Am. J. Physiol., 265, R41-R48.

Larade, K., and Storey, K.B. 2009. Living without oxygen: anoxia-responsive gene expression and regulation. Curr. Genom., 10, 76-85.

Lewis, B.P., Shih, I., Jones-Rhoades, M., Bartel, D., and Burge, C. 2003. Prediction of mammalian microRNA targets. Cell, 115, 787-798.

Lewis, B.P., Burge, C.B., and Bartel, D.P. 2005. Conserved seed pairing, often flanked by adenosines, indicates that thousands of human genes are microRNA targets. Cell, 120(1), 15-20.

Leung, A.K.L., Calabrese, J.M., and Sharp, P.A. 2006. Quantitative analysis of Argonaute protein reveals microRNA-dependent localization to stress granules. P. Natl. Acad. Sci. USA, 103(48), 18125-18130.

Leung, K., and Sharp, P. 2010. MicroRNA functions in stress responses. Mol. Cell, 40, 205-215.

Lin, S., Wang, W., Wilson, G.M., Yang, X., Brewer, G., Holbrook, N.J., and Gorospe, M. 2000. Down-regulation of cyclin D1 expression by prostaglandin $A_{2}$ is mediated by enhanced cyclin D1 mRNA turnover. Mol. Cell. Biol., 20(21), 7903-7913.

Linsley, P., Schelter, J., Burchard, J., Kibukawa, M., Martin, M., Bartz, S., Johnson, J., Cummins, J., Raymond, C., Dai, H., Chau, N., Cleary, N., Jackson, A., Carleton, M., and Lim, L. 2007. Transcripts targeted by the microRNA-16 family cooperatively regulate cell cycle progression. Mol. Cell Biol., 27, 2240-2252.

Liu, J., Valencia-Sanchez, M.A., Hannon, G.J., and Parker, R. 2005. MicroRNAdependent localization of targeted mRNAs to mammalian P-bodies. Nature Cell Biol., 7, 719-723. 
Liu, J., Chen, G., Feng, L., Zhang, W., Pelicano, H., Wang, F., Ogasawara, M.A., Lu, W., Amin, H.M., Croce, C.M., Keating, M.J., and Huang, P. 2013. Loss of p53 and altered miR15-a/16-1 in the MCL-1 pathway in CLL : Insights from TCL1Tg:p53-/- mouse model and primary human leukemia cells. Leukemia, doi: 10.1038/leu.2013.125.

Liu, Q., Fu, H., Sun, F., Zhang, H., Tie, Y., Zhu, J., Xing, R., Sun, Z., and Zheng, X. 2008. MiR-16 family induces cell cycle arrest by regulating multiple cell cycle genes. Nucleic Acids Res., 36, 5391-5404.

Matsushime, H., Quelle, D.E., Shurtleff, S.A., Shibuya, M., Sherr, C.J., Kato, J.Y. 1994. D-type cyclins-dependent kinase activity in mammalian cells. Mol. Cell. Biol., 14(3), 2006-2076.

Masamha, C.P., and Benbrook, D.M. 2009. Cyclin D1 degradation is sufficient to induce G1 cell cycle arrest despite constitutive expression of cyclin E2 in ovarian cancer cells. Cancer Res., 69(16), 6565-6572.

Mazia, D. 1962. Biochemistry of the dividing cell. Annu. Rev. Biochem., 30, 669-688.

Morin, P.J., Dubuc, A., and Storey, K.B. 2008. Differential expression of microRNA species in organs of hibernating ground squirrels: a role in translational suppression during torpor. BBA-Gene Regul. Mech., 1779(10), 628-633.

Nishi, K., Inoue, H., Schnier, J.B., and Rice, R.H. 2009. Cyclin D1 Downregulation is important for permanent cell cycle exit and initiation of differentiation induced by anchorage-deprivation in human keratinocytes. J. Cell Biochem., 106(1), 63-72.

O'Connor, C. 2008. Cell Division: Stages of Mitosis. Nature Ed., 1(1).

Pahl, H.L. 1999. Activators and target genes of Rel/NF-kappaB transcription factors. Oncogene, 18(49), 6853-6866.

Rehmsmeier, M., Steffen, P., Hochsmann, M., and Giegerich, R. 2004. Fast and effective predication of microRNA/target duplexes. RNA, 10, 1507-1517.

Rimokh, R., Berger, F., Bastard, C., Klein, B., French, M., Archimbaud, E., Rouault, J.P., Santa Lucia, B., Duret, L., and Vuillaume, M. 1994. Rearrangement of CCND1(BCL1/PRAD1) 3' untranslated region in mantle-cell lymphomas and $\mathrm{t}(11 \mathrm{q} 13)$-associated leukemias. Blood, 83, 3689-96.

Rosenwald, I.B., Lazaris-Karatzas, A., Sonenberg, N., and Schmidt, E.V. 1993. Elevated levels of cyclin D1 protein in response to increased expression of eukaryotic initiation factor 4E. Mol. Cell. Biol., 13(12), 7358-7363.

Roufayel, R., Biggar, K.K., and Storey, K.B. 2011. Regulation of cell cycle components during exposure to anoxic and dehydration stress in the wood frog, Rana sylvatica. J. Exp. Zool., 313A(8), 487-494.

Safdar, A., Abadi, A., Akhtar, M., Hettinga, B.P., and Tarnopolsky, M.A. 2009. miRNA in the regulation of skeletal muscle adaptation to acute endurance exercise in C57Bl/6J male mice. PLoS ONE., 4(5), e5610. 
Schier, A., and Giraldez, A. 2006. MicroRNA function and mechanism: insights from zebrafish. Cold Spring Harb. Sym., 121, 195-203.

Sherr, C.J. 1995. D-type cyclins. Trends Biochem. Sci., 20, 187-190.

Shi, Y., and Jin, Y. 2010. MicroRNA in cell differentiation and development. Sci. China C. Life Sci., 52(3), 205-211.

Singh, J., and Nagaraju, J. 2008. In silico prediction and characterization of microRNAs from red flour beetle (Tribolium castaneum). Insect Mol. Biol., 17, 427-436.

Stacey, D.W. 2003. Cyclin D1 serves as a cell cycle regulatory switch in actively proliferating cells. Curr. Opin. Cell Biol., 15, 158-163.

Storey, K.B. 2007. Anoxia tolerance in turtles: metabolic regulation and gene expression. Comp. Biochem. Physiol. A, 147, 263-276.

Storey, K.B., and Storey, J.M. 2004. Metabolic rate depression in animals: transcriptional and translational controls. Biological Rev., 79(1), 207-233.

Tarn, W.Y., and Lai, M.C. 2011. Translational control of cyclins. Cell Division, 6, 5.

Tan, J., Fan, L., Mao, J.J., Chen, B., Zheng, L., Zhang, T., Li, T., Duan, J., Duan, Y., Jin, Z., and Kuang, W. 2012. Restoration of miR-34a in p53 deficient cells unexpectedly promotes the cell survival by increasing NFkB activity. J. Cell. Biochem., 113(9), 2903-2908.

Tarasov, V., Jung, P., Verdoodt, B., Lodygin, D., Epanchintsev, A., Menssen, A., Meister, G., and Hermeking, H. 2007. Differential regulation of microRNAs by p53 revealed by massively parallel sequencing: miR-34a is a p53 target that induces apoptosis and G1-arrest. Cell Cycle, 6(13), 1586-1593.

Watanabe, Y., Yachie, N., Numata, K., Saito, R., Kanai, A., and Tomita, M. 2006. Computational analysis of microRNA targets in Caenorhabditis elegans. Gene, 365, 2-10.

Wiestner, A., Tehrani, M., Chiorazzi, M., Wright, G., Gibellini, F., Nakayama, K., Liu, H., Rosenwald, A., Muller-Hermelink, H.K., Ott, G., Chan, W.C., Greiner, T.C., Weisenburger, D.D., Vose, J., Armitage, J.O., Gascoyne, R.D., Connors, J.M., Campo, E., Montserrat, E., Bosch, F., Smeland, E.B., Kvaloy, S., Holte, H., Delable, J., Fisher, R.I., Grogan, T.M., Miller, T.P., Wilson, W.H., Jaffe, E.S., and Staudt, L.M. 2007. Point mutations and genomic deletions in CCND1 create stable truncated cyclin D1 mRNAs that are associated with increased proliferation rate and shorter survival. Blood, 109, 4599-606.

Willmore, W.G., and Storey, K.B. 1997. Antioxidant systems and anoxia tolerance in a freshwater turtle Trachemys scripta elegans. Mol. Cell. Biochem., 170, 177-185.

Wu, C.W. and Storey, K.B. 2012. Pattern of cellular quiescence over the hibernation cycle in liver of thirteen-lined ground squirrels. Cell Cycle, 6(7), 1714-1726.

Zhang, R., and Adams, P.D. 2007. Heterochromatin and its relationship to cell senescence and cancer therapy. Cell Cycle, 6(7), 784-789. 
Zhang, J. and Storey, K.B. 2012. Cell cycle regulation in the freeze tolerant wood frog, Rana sylvatica. Cell Cycle, 11(9), 1727-1742.

Zhang, J., Biggar, K.K., and Storey, K.B. 2013. Regulation of p53 by reversible posttranscriptional and post-translational mechanisms in liver and skeletal muscle of an anoxia tolerant turtle, Trachemys scripta elegans. Gene, 513(1), 147-155. 
Table 5.1. Determination of RNA binding structure and theoretical minimum free energy (mfe) values using RNAhybrid. Shaded regions indicate the conserved seed-pairing between the microRNA and its target.

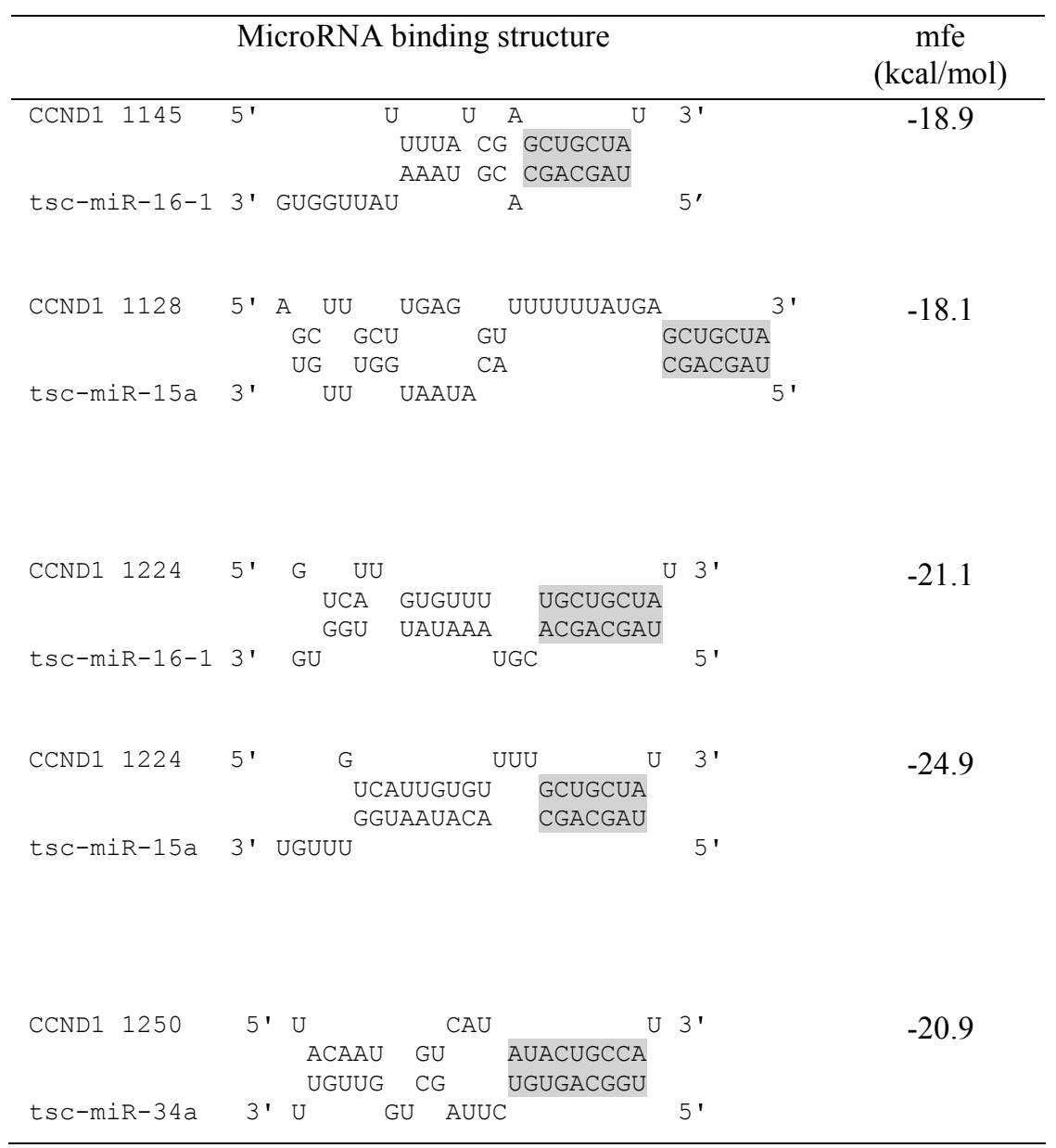




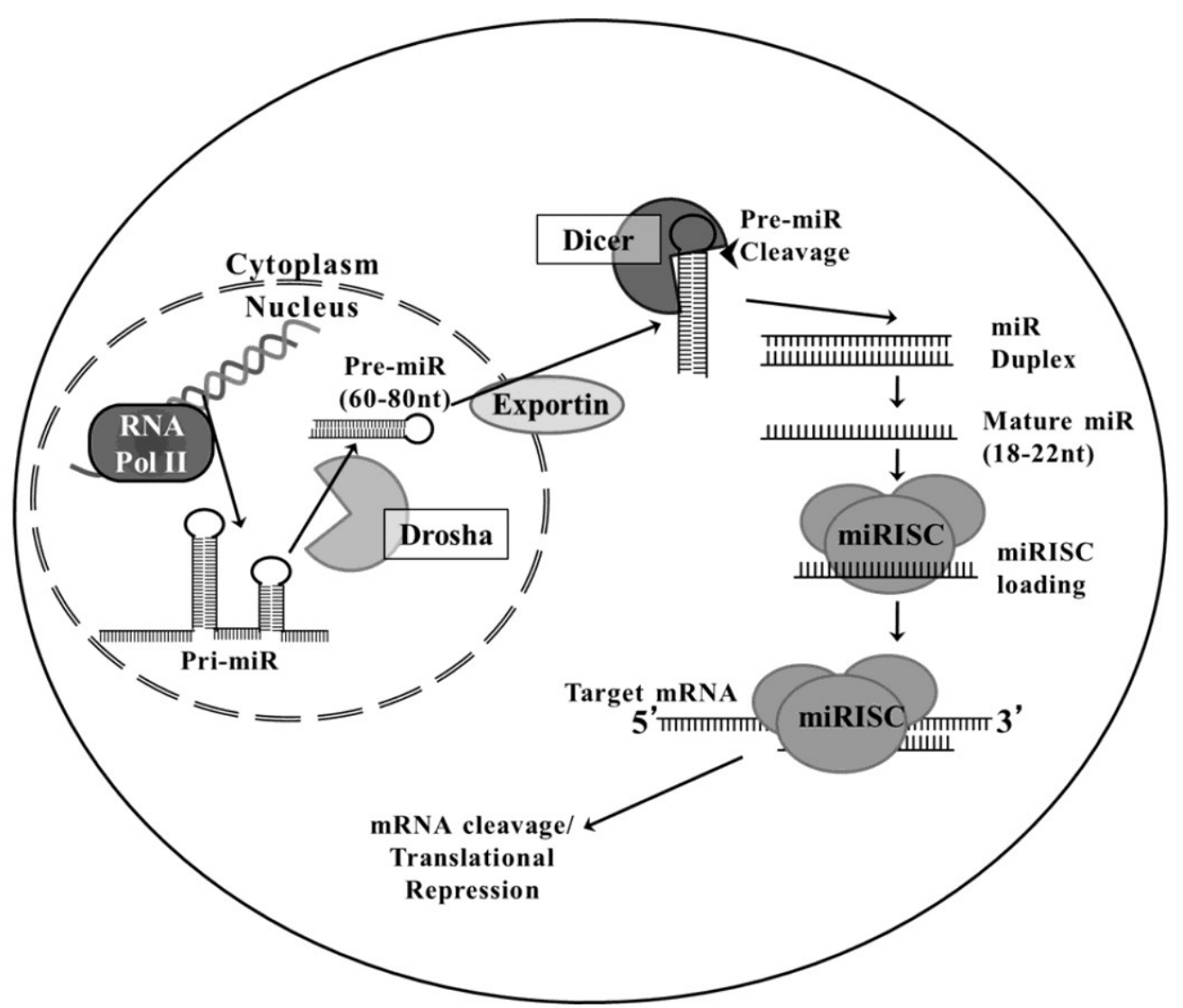

Figure 5.1. MicroRNA biogenesis. MicroRNAs begin in the nucleus where they are processed and exported into the cytoplasm. In the cytoplasm, they form complexes with Ago and create the miRISC. It is this RISC complex that binds to mRNA 3' UTR and inhibits translation. 


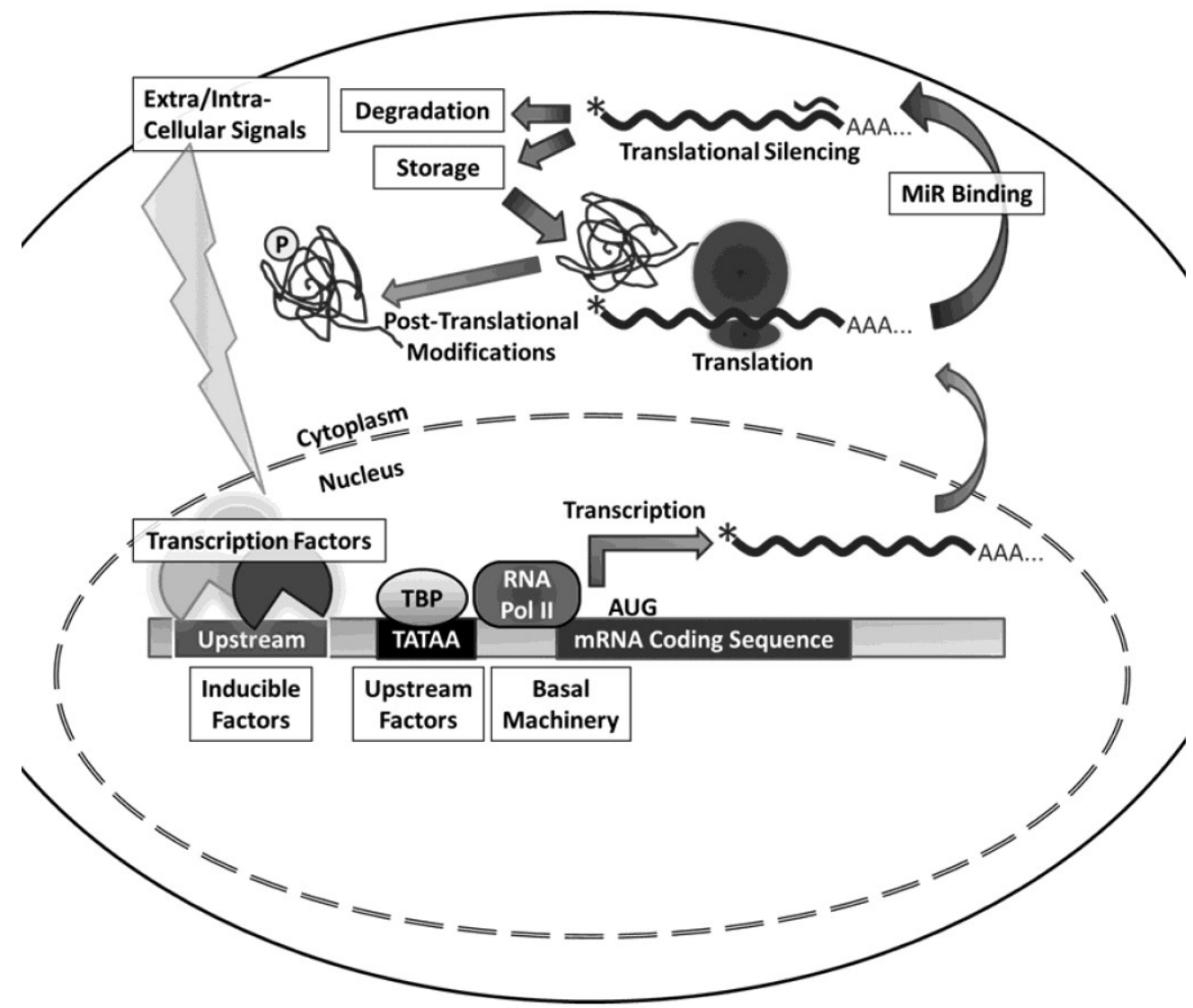

Figure 5.2. Schematic representation of microRNA function. Mature microRNAs regulate protein translation by the 3' UTR of a conserved target mRNA. The degree of complementarity between the microRNA and target, can dictate a fate of either mRNA storage (imperfect) or degradation (perfect). 


\section{ENVIRONMENTAL STRESS}

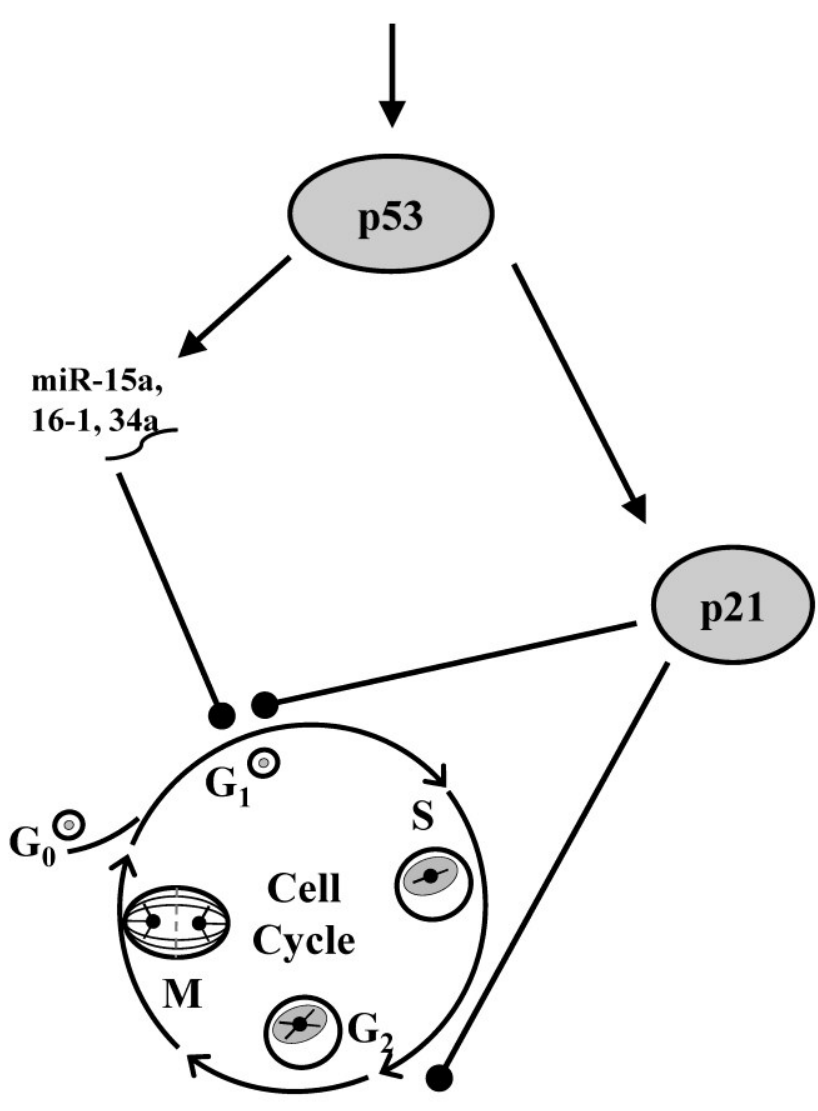

Figure 5.3. Effect of $\mathrm{p} 53$ and its downstream targets (p21, miR-16-1, miR-15a and miR-34a) on the cell cycle. Increased transcriptional activity of p53 increases the expression of CKI (p21) and microRNA targets (miR-16-1, miR-15a and miR-34a) that inhibit the cell cycle. 
A)

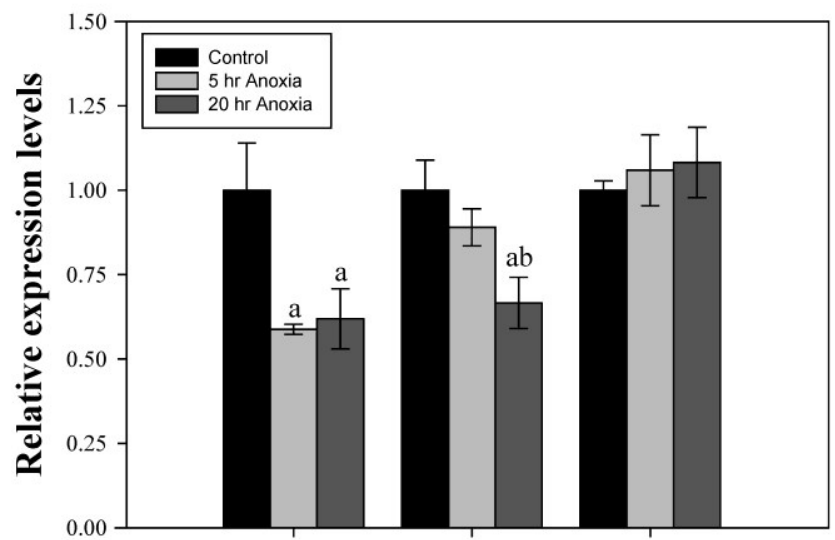

B)

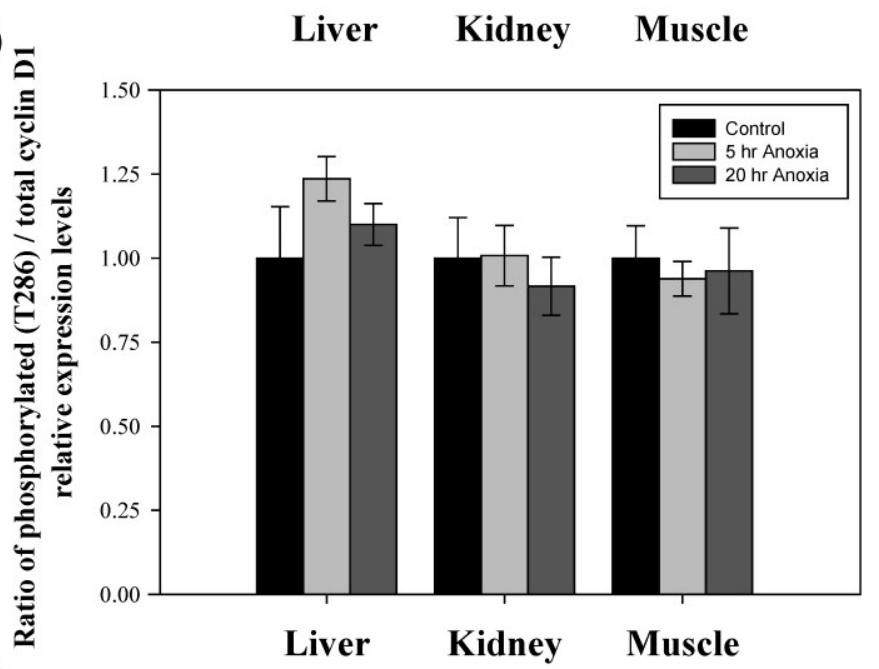

C)

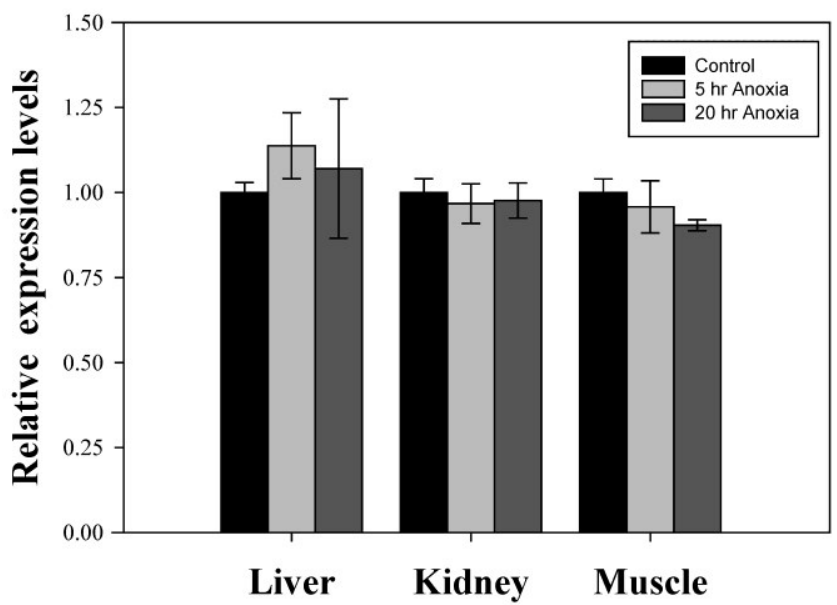

Figure 5.4. Effect of 5 and $20 \mathrm{~h}$ of anoxic submergence on relative protein, phosphorylation and mRNA levels of Cyclin D1 in T. s. elegans. Histograms show normalized expression levels under control and anoxic conditions of (A) total protein levels of Cyclin D1, (B) the ratio of total Cyclin D1 protein to pCyclin D1(Thr284) and (C) transcript levels of Cyclin D1. Representative images are presented in Chapter 2 (see Figure 2.4). Data are means \pm S.E.M., $n=3-4$ independent trials. a: Significantly different from the corresponding control $(\mathrm{P}<0.05)$; b: significantly different from the $5 \mathrm{~h}$ anoxic value $(\mathrm{P}<0.05)$. 
1 GUUUAUUUCA AACCCACCGU UCAUGAUCGC AGCUGGgAgU GUGGUAGCAG CUGUGCAAGg 61 CCUUCACCUG GGAAACACUA ACACUUUCCU CUCCUAUCAA UGUCUCACAC AUUUCCUAUC 121 ACAAGUUAUC AAAUGUGACC CGGAUUGUUU ACGAGCUUGC CAAGAACAGA UUGAAUCCCU 181 CCUCGAAUCC AGUCUACGCC AGGCACAGCA ACACAACAUA UCUUCAGAAA CAAAGACUGU 241 UGAGGAUGAA GCAGACCUUU CCUGCACACC CACUGAUGUG CGAGACGUGA ACAUUUAAGA 301 GGACUUCUAC AAAUCGgUUU GUUUGGCAAU GAGAGCAGAC AAAGAAAgGG CAUCUGAGAA 361 GGAAUCAGCA UCAGGAUCUC UCCCCCAGAA ACCCUUUUCU CCAGGACAUU UUUAUACCAG 421 AagGGAAAGC UUCACUCUUG UUAUUUUUUU CCUUGCUCUU UCUCCCUUCC AUCUGUGACU 481 UCAAACAAAC AAAAAAUACC CCAAAACUGU CUUAAAAAAA AAAAAAAAAA CUACUAAUCG 541 AACCGGGGCC CAAAAAGCAU AgCUUUAAUG CAgUUCUAGg CUUUUCUGUA UCUUGCUUGC

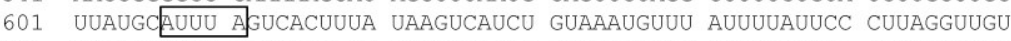
661 UAUCCUCUUC ACCUUAUCAA UGGUUCAUGU AACCUUUACG UUAAAGAGAA AAUGGAAUGG

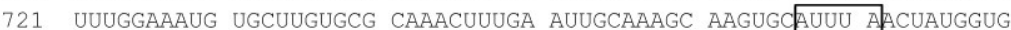
781 UUAGAGGAAA GGUGGAAGGU UGUGUUCCAG UGAGAGAAAU UGCAUUCACA AAUUGCCAGG 841 AUAUCUUCCU AUCCUUUUCU AUAGAAAAGU UAGGGGCCUU AAAGGUUCAC GUAUAGGACA 901 CAUAGUUUAA GGAUUGUCUC UAGAUGUUUU ACAACUGAAG GUUUUUAAAC ACUAAAAUAU 961 AUA AUUUA UA GUUAAGGCAA AAAGAAUAUU UAUUGCAGAG GAUGUUCAUA AGGCCAGUAU 1021 GAUUUGUAAA AUAA AUUUAG CUACAAAUAG AAAAUUUGCA CAUCUUGGCU AUGUAUCUUU

1081 UUAUUAUUAU UUUAGAAAGU AGCUGAAGGG ACGUGGACAU AGCUUGCUUG AGGUUUUUUU

1141 AUCGAGCUGC UAUGGAUAAA UUCCCAGCAU GAUAAAUUCC CAGCAUGGUU UGAAAAAAAU 1201 UAACAUUGCU UUCUGUAUCU UUGUCAUUGU GUUUUGCUGC UAUUUUUUAU ACAAUGUCAU

1261 AUACUGCCAU GUUAUAGGUU UUAAUUUUCU CAUAGAAAAU UAUAUUAUUG ACAUCAUGUG 1321 AUCAAUUUUU ACUUAAUGUG AUUAUUACUG CUCUAUUCCA AAAAGGUUCC UGUUUCACAA 1381 UACCUCAUAC UUCAGUUAAC CUGUCUGAUC UGGGGUUUAU UAUCAUAGUA GUUUUAAGAU 1441 GUGUUAUUAG CCACAUUGAU AUAUGAAAGG UGCAUUAUAA UAUAACUCUU AUAUGCCACU 1501 UgGUGGUGUU UCCCAUCAGC UGGGACUCAU CAUUUGGUAU GAUAGGUUAU CUUCUGGAAC 1561 UCUAAGUAUU UUgUgUGUUU AAUCUGUUAU AAUAUACUAG GGUUUUUUGU UUUGUUUUUU 1621 GUUAAUUAUA GCAUAAUGCU AUUUA

Figure 5.5. Identification of regulatory elements within the Cyclin D1 3' UTR. AU-sites are identified with boxes and microRNA binding sites are underlined. MicroRNA binding sites were identified using TargetScan v.5.2. 


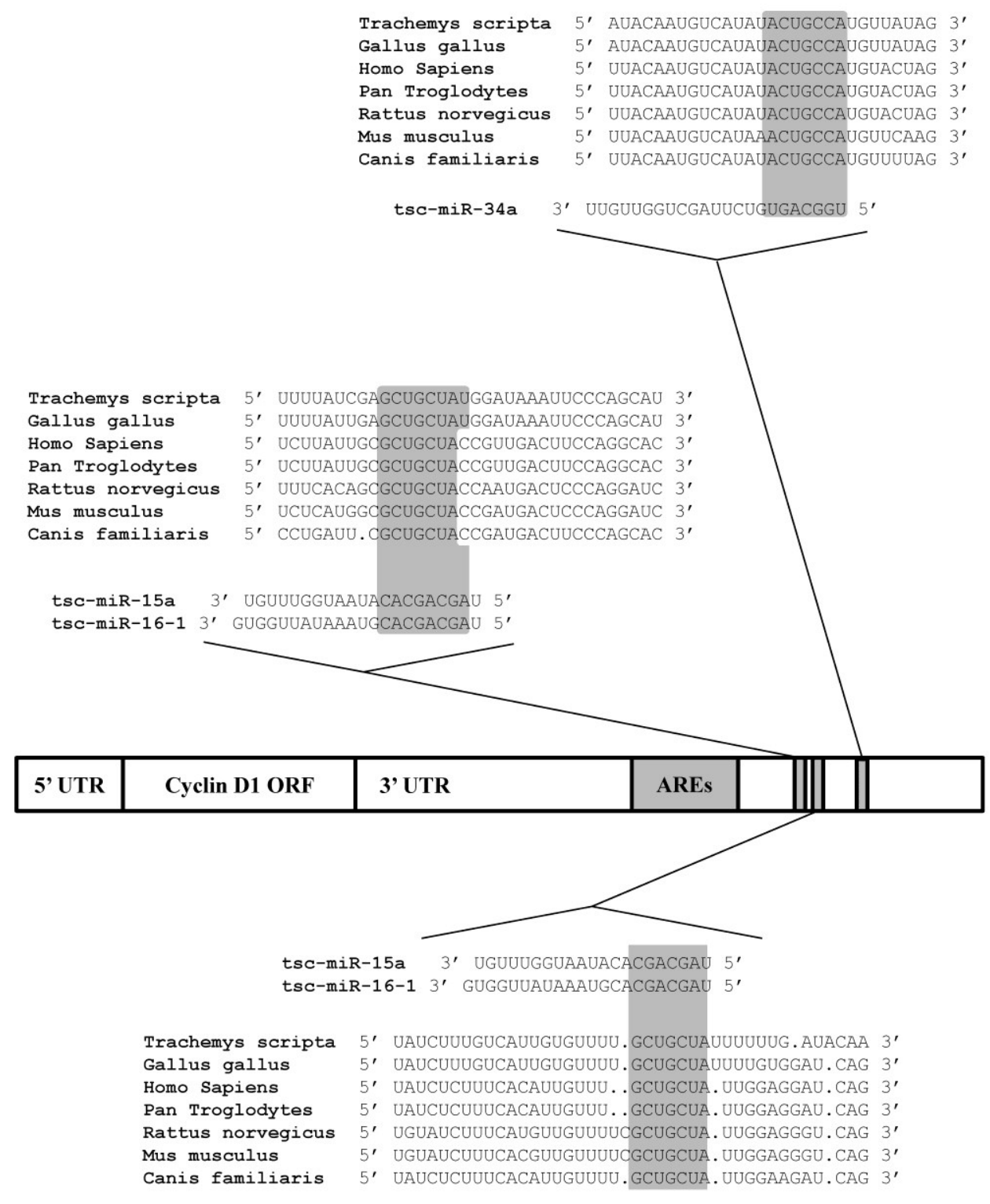

Figure 5.6. Theoretical binding of tsc-miRNA-16-1, tsc-miR-15a and tsc-miR-34a to conserved binding regions in the cyclin D1 3' UTR of the turtle. Conservation analysis of the microRNA binding sites from thered-eared slider turtle (Trachemys scripta elegans), chicken (Gallus gallus), zebra fish (Danio rerio), human (Homo sapiens), common chimpanzee (Pan troglodytes), brown rat (Rattus norvegicus), house mouse (Mus musculus) and the domestic dog (Canis familiaris). The seed region sequence (shaded) shows high conservation between the five sequences for all binding sites. ARE identifies the relative location of the AU-rich element found within the 3' UTR of turtle cyclin D1. 
A)

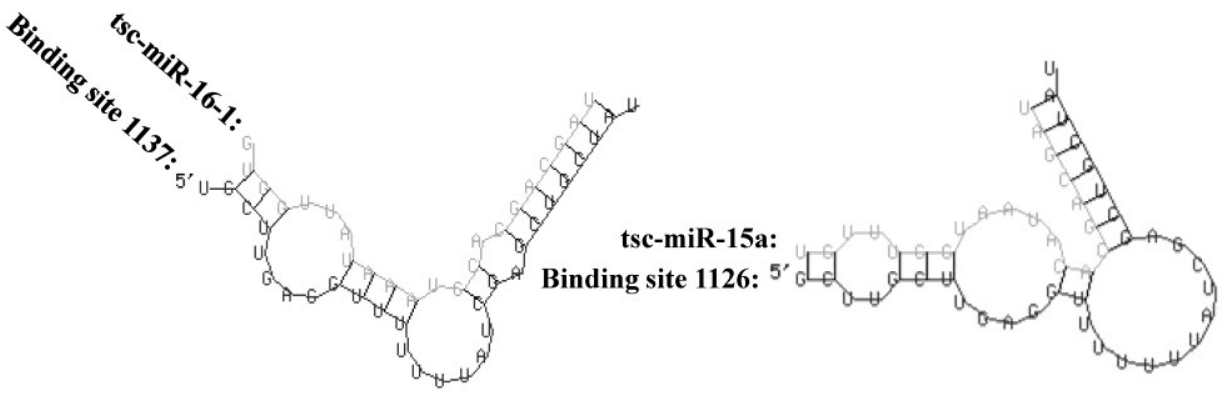

B)

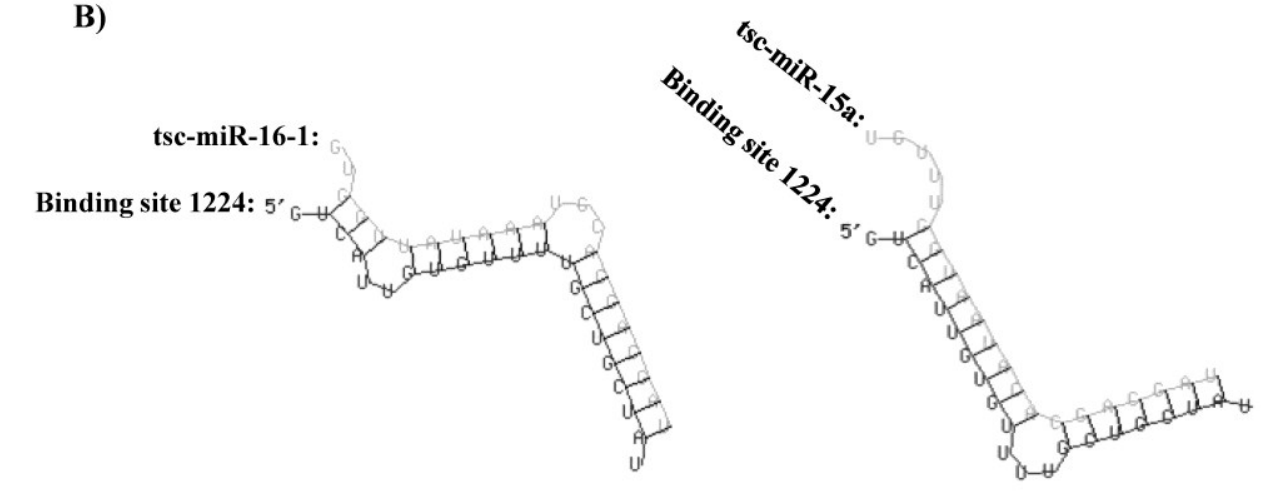

C)

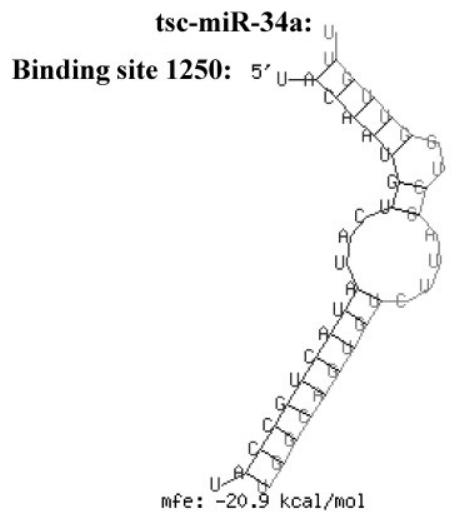

Figure 5.7. Theoretical binding of tsc-miRNA-16-1, tsc-miR-15a and tsc-miR-34a to conserved binding regions in the cyclin D1 3' UTR of the turtle. Predicted binding structures of both tsc-miR-161 and tsc-miR-15a binding to the 3'UTR of cyclin D1, determined from the RNAhybrid program. 
A)
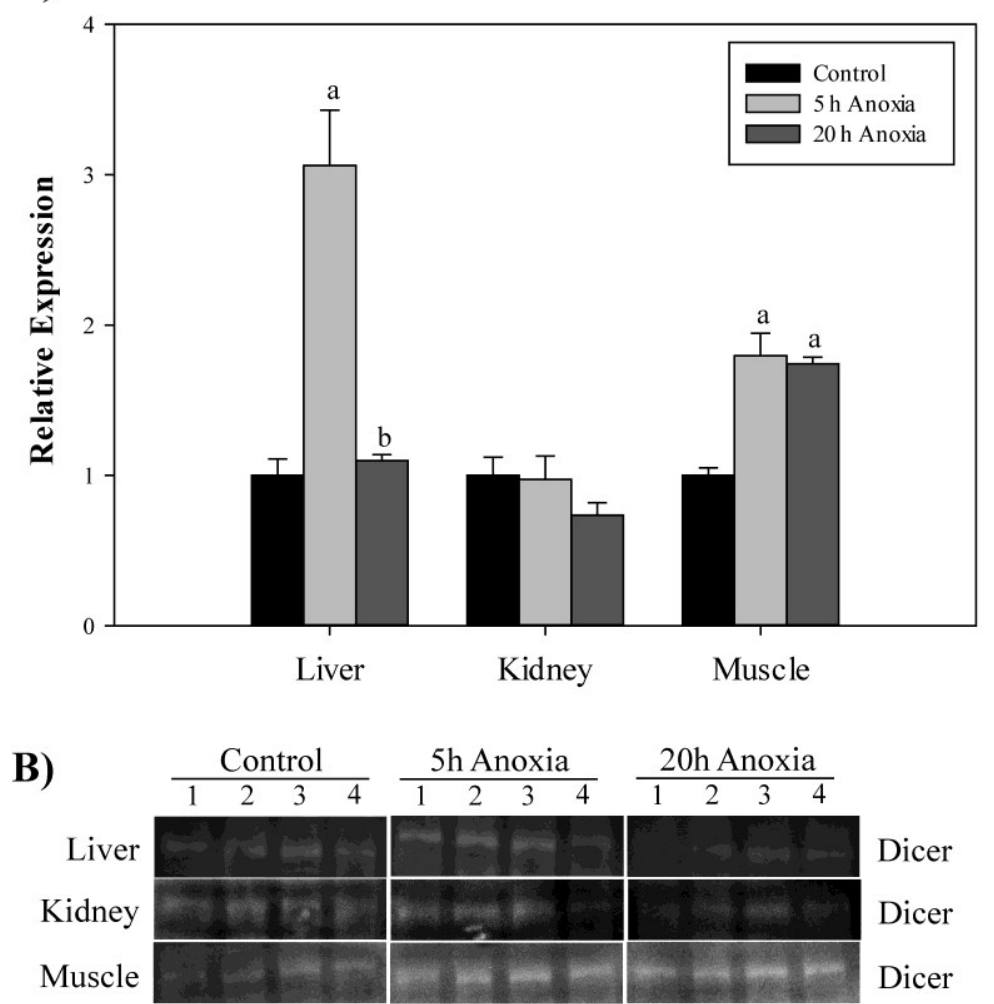

Figure 5.8. Effect of anoxic stress on the relative expression of Dicer protein in the liver, kidney and skeletal muscle from control and anoxic ( 5 and $20 \mathrm{~h}$ ) turtles. (A) Histogram shows normalized expression levels for control versus 5 and $20 \mathrm{~h}$ anoxic conditions. (B) Representative bands of Dicer immunoblotting. Data are means \pm S.E.M., $n=3-4$ independent trials. a: Significantly different from the corresponding control $(\mathrm{P}<0.05)$; b: significantly different from the $5 \mathrm{~h}$ anoxic value $(\mathrm{P}<0.05)$. 

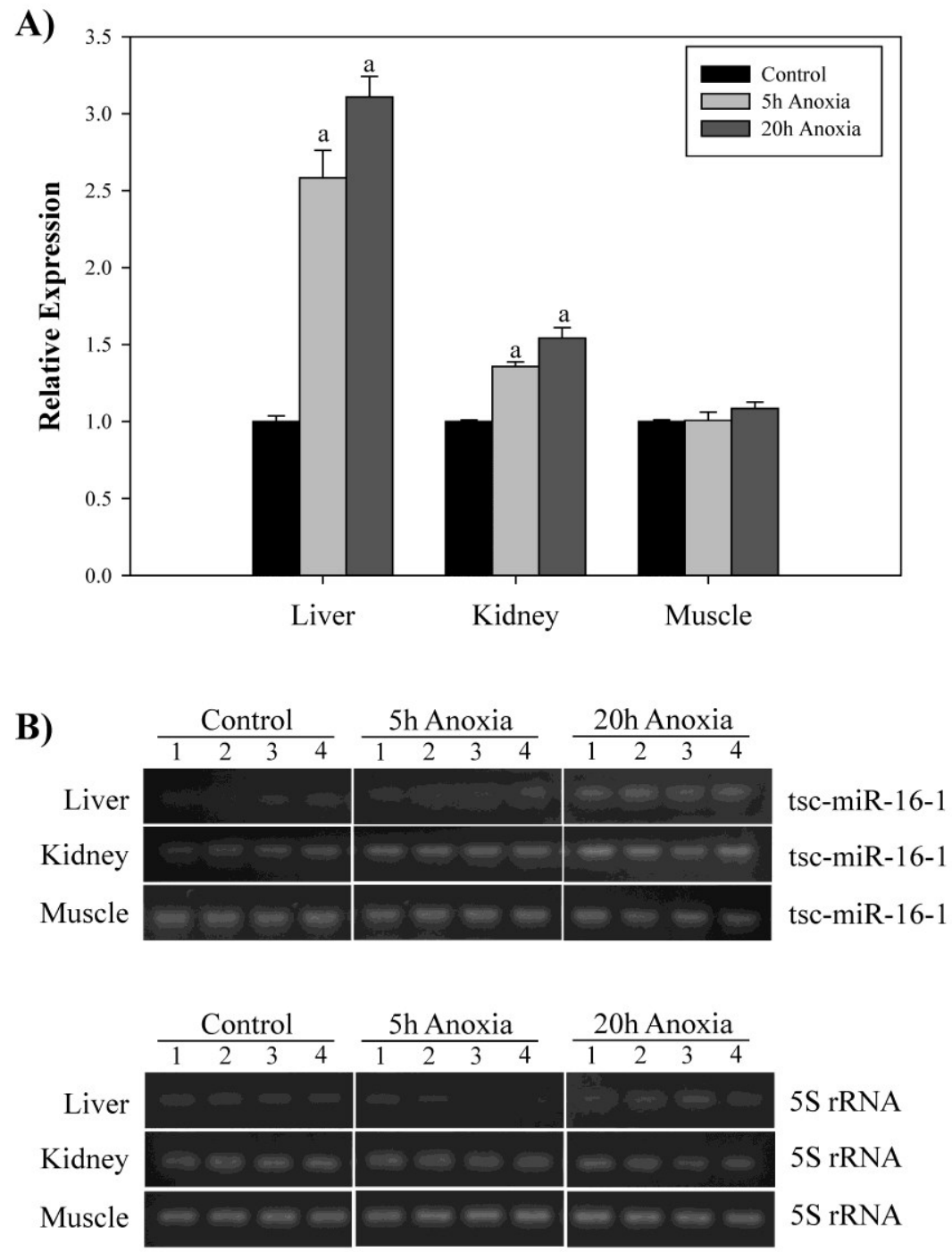

Figure 5.9. Effect of anoxic stress on the relative expression of tsc-miR-16-1 in the liver, kidney and skeletal muscle from control and anoxic ( 5 and $20 \mathrm{~h}$ ) turtles. (A) Histogram shows normalized expression levels for control versus 5 and $20 \mathrm{~h}$ anoxic conditions. (B) Representative bands show RNA transcript levels amplified by RT-PCR. Band intensities from the RT-PCR samples were normalized with $5 \mathrm{~S}$ rRNA bands from the corresponding sample. Data are means \pm S.E.M., $n=3-4$ independent trials. a: Significantly different from the corresponding control $(\mathrm{P}<0.05)$; b: significantly different from the $5 \mathrm{~h}$ anoxic value $(\mathrm{P}<0.05)$. 
A)

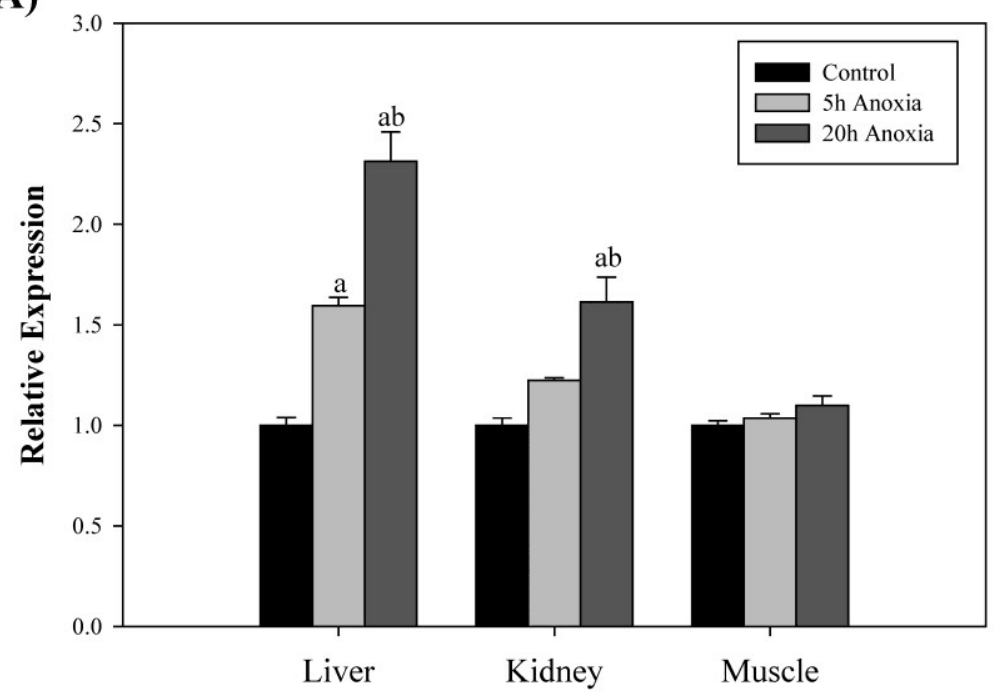

B)
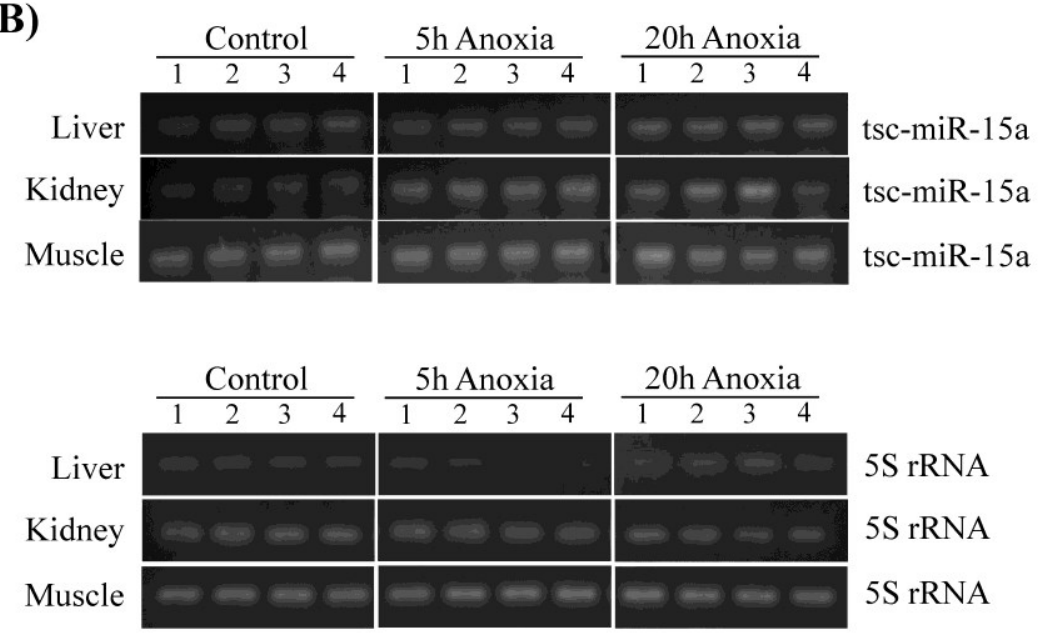

Figure 5.10. Effect of anoxic stress on the relative expression of tsc-miR-15a in the liver, kidney and skeletal muscle from control and anoxic ( 5 and $20 \mathrm{~h}$ ) turtles. (A) Histogram shows normalized expression levels for control versus 5 and $20 \mathrm{~h}$ anoxic conditions. (B) Representative bands show RNA transcript levels amplified by RT-PCR. Band intensities from the RT-PCR samples were normalized with $5 \mathrm{~S}$ rRNA bands from the corresponding sample. Data are means \pm S.E.M., $n=3-4$ independent trials. a: Significantly different from the corresponding control $(\mathrm{P}<0.05)$; $\mathrm{b}$ : significantly different from the $5 \mathrm{~h}$ anoxic value $(\mathrm{P}<0.05)$. 

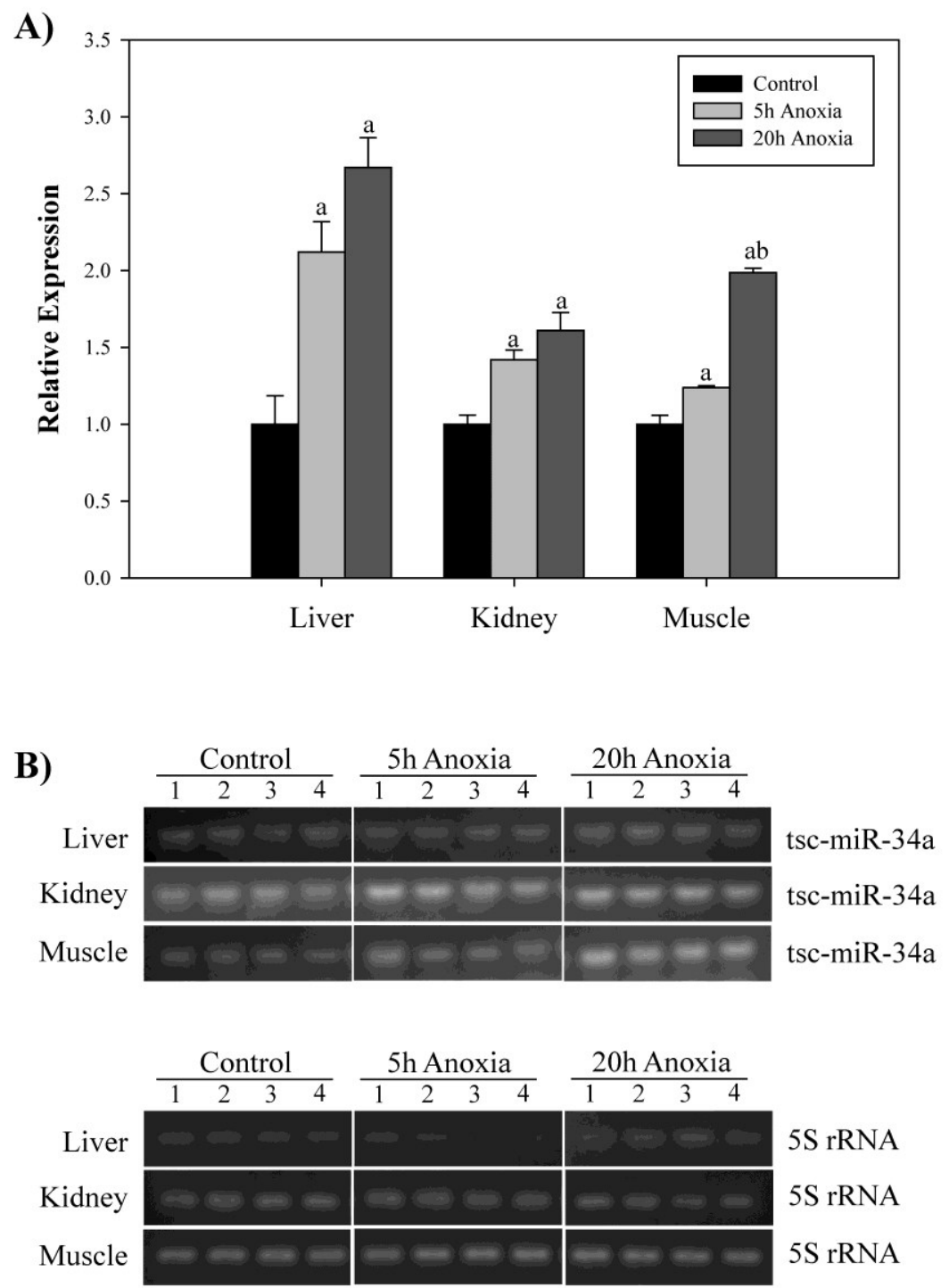

Figure 5.11. Effect of anoxic stress on the relative expression of tsc-miR-34a in the liver, kidney and skeletal muscle from control and anoxic $(5$ and $20 \mathrm{~h})$ turtles. (A) Histogram shows normalized expression levels for control versus 5 and $20 \mathrm{~h}$ anoxic conditions. (B) Representative bands show RNA transcript levels amplified by RT-PCR. Band intensities from the RT-PCR samples were normalized with $5 \mathrm{~S}$ rRNA bands from the corresponding sample. Data are means \pm S.E.M., $n=3-4$ independent trials. a: Significantly different from the corresponding control $(\mathrm{P}<0.05)$; $\mathrm{b}$ : significantly different from the $5 \mathrm{~h}$ anoxic value $(\mathrm{P}<0.05)$. 


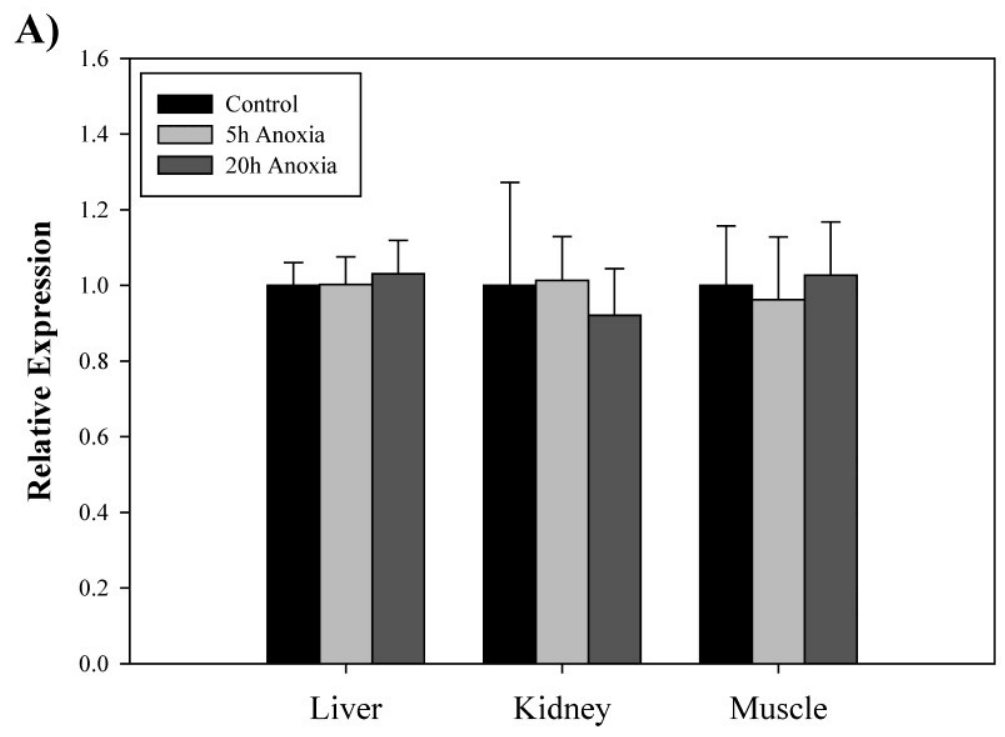

B)

\begin{tabular}{|c|c|c|c|c|c|c|c|c|c|c|c|c|}
\hline & \multicolumn{4}{|c|}{ Control } & \multicolumn{4}{|c|}{ 5h Anoxia } & \multicolumn{4}{|c|}{ 20h Anoxia } \\
\hline & 1 & 2 & 3 & 4 & 1 & 2 & 3 & $\overline{4}$ & 1 & 2 & 3 & 4 \\
\hline Liver & 8 & (6) & 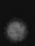 & $c$ & 6 & 8 & $\bullet$ & 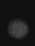 & 6 & 8 & $\boldsymbol{\theta}$ & * \\
\hline Kidney & 0 & 웅 & 6 & $\mathrm{~s}$ & $\bullet$ & - & $\bullet$ & 0 & - & $\bullet$ & $\bullet$ & e \\
\hline Muscle & o & $\theta$ & $\theta$ & $c$ & 争 & 당 & $\theta$ & 8 & e & $\theta$ & e & $e$ \\
\hline
\end{tabular}

C)

\begin{tabular}{|c|c|c|c|c|c|}
\hline \multicolumn{6}{|c|}{ Total RNA (ug) } \\
\hline $\begin{array}{ll}0.1 & 0.5\end{array}$ & 12 & 3 & 5 & 10 & 15 \\
\hline 0 & 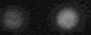 & 6 & 8 & $\varepsilon$ & 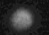 \\
\hline
\end{tabular}

Figure 5.12. Effect of anoxic stress on the relative abundance of poly-adenylated mRNA from the liver, kidney and skeletal muscle of control and anoxic (5 and $20 \mathrm{~h}$ ) turtles. (A) Histogram shows normalized ploy-adenylated mRNA levels for control versus 5 and $20 \mathrm{~h}$ anoxic conditions. (B) Representative dot-blots show poly-adenylated RNA levels. (C) Dose-response relating the total amount of RNA to poly-adenylated luminescent signal using RNA from control liver $(n=1)$. Data are means \pm S.E.M., $n=3-4$ independent trials. a: Significantly different from the corresponding control $(\mathrm{P}<0.05)$; b: significantly different from the $5 \mathrm{~h}$ anoxic value $(\mathrm{P}<0.05)$. 


\section{CHAPTER 6}

\section{GENERAL DISCUSSION}




\subsection{General discussion}

Oxygen deprivation is extremely harmful for most organisms. In aerobic animals, the complete catabolism of all fuel sources relies on oxidative phosphorylation, in which oxygen plays a crucial role as the final electron acceptor. When oxidative phosphorylation is inhibited, anaerobic glycolysis becomes the sole energy producer of the cell. However, this pathway has several limitations, including poor ATP yield, toxic end products (lactic acid), and a quick depletion of fuel sources. Therefore, most aerobic life forms are fairly intolerant of oxygen limitation and sustain significant damage and/or death when subjected to anoxia.

In contrast, some animals can survive quite well with or without oxygen. Among terrestrial vertebrates, the best facultative anaerobes are turtles belonging to the Trachemys and Chrysemys genera, including the red-eared slider, T. scripta elegans and the painted turtle $C$. picta. These turtles have developed several mechanisms to deal with the problems posed by usage of anaerobic glycolysis as their primary ATP producing pathway (Storey, 2007). The end product of glycolysis, lactate, is collected and stored in the shell and bones of the turtles, and the protons generated are neutralized by bicarbonate released from shell and bone, preventing acidification (Figure 1.2). In addition, huge glycogen stores in the liver ensure adequate fuel to supply glycolysis for extended periods of anaerobic life. Finally, to deal with the problem of insufficient ATP production by glycolysis, turtles significantly depress their metabolic rate to $10-20 \%$ of the corresponding aerobic rate at the same body temperature, as well as reorganize their metabolic priorities, in order to meet the energy needs of vital cell functions in the anoxic state (Figure 1.3) (Storey, 1996; Storey, 2007). 
While research on turtle anoxia tolerance has primarily been focused on the physiological and biochemical processes involved, little has been done to investigate the state of the cell cycle in response to anoxia in turtles. The goal of my thesis was to address this problem by using T. scripta elegans as the model and examining the responses of three key aspects of cell cycle regulation: (1) cell cycle initiation by signaling pathways, (2) protein and mRNA expression of key cell cycle regulatory targets, and (3) post-transcriptional regulation by mature microRNA. This thesis used a variety of different techniques, including immunoblotting, subcellular fractionation, semi-quantitative RT-PCR, 3' RACE, microRNA-specific RT-PCR, RNA dot-blots, EMSAs, DNA-bound protein complex formation and transcription factor ELISAs to examine the responses to anoxia of several important proteins and pathways at various levels of regulation. The levels of regulation examined included transcript and protein expression, subcellular localization, post-translational modifications, post-transcriptional regulation by microRNA, protein-protein interactions and DNA-binding activity of protein complexes.

As a genome sequence for the red-eared slider turtle was not available during the course of these studies, to examine two major parameters of this thesis, several new techniques needed to be created, developed and validated. To sequence mature microRNAs and evaluate their expression in turtle tissues, a new amplification and sequencing technique was developed. Since mature microRNAs are extremely small in size ( $\sim 22 \mathrm{bp})$, amplification and sequencing required the addition of a nucleotide adapter sequence (60 bp) (Biggar et al., 2011). This adapter sequence maintains microRNAspecificity by forming a stable stem-loop structure at temperatures below $60{ }^{\circ} \mathrm{C}$, allowing 
access only to a small microRNA-specific binding region (see Appendix K for both stemloop structure and amplification mechanism). Additionally, the DNA-bound protein complex ELISA technique was developed to evaluate the composition and transcriptional activity of DNA binding complexes (see Appendix $\mathrm{J}$ for development and validation). The development of this technique was crucial as the use of traditional co-IP protocols in the immunoprecipitation of proteins bound in complex would yield irrelevant results; there is no indication if these complexes have an influence on transcriptional activity (i.e. DNA bound vs. free). Traditional analyses of transcriptionally active protein complexes utilize co-immunoprecipitation and chromatin immunoprecipitation; the cross-linking of proteins to each other and to the DNA at which it is located. These techniques, although suitable for well characterized animal models, are extremely difficult to validate for nonsequenced animals. The inability to (1) determine the DNA sequence to which the protein complex is bound, and (2) the likelihood of this technique to generate a high degree of false positives, makes chromatin immunoprecipitation a costly alternative to the outlined method of DNA-bound protein complex ELISA. Overall, the results of this thesis point to multiple previously unknown mechanisms that play a role in cell cycle regulation during extended periods of oxygen limitation in an anoxia tolerant vertebrate.

\subsection{Anoxia tolerance and the stress response}

Complete oxygen deprivation can present several problems to the cell. Besides the aforementioned stress on metabolism, oxygen deprivation is characterized by an altered redox environment, as well as decreased cellular $\mathrm{pH}$ resulting from lactic acid production. Turtles escape anoxia-induced cellular damage by suppressing, rebalancing and 
reprioritizing the rates of ATP-utilizing and ATP-generating processes so that they can sustain long term viability without oxygen. Although it is clear that anoxic survival relies on the suppression of various ATP consuming processes, the state of the cell cycle in anoxia tolerant organisms remained unknown. The demanding energetics of mitosis provided the intriguing suggestion that the cell cycle must arrest in proliferating tissues in order to facilitate metabolic rate depression and that cell cycle regulation must play an important role in cellular responses to environmental stresses. Therefore, an important aspect of anoxia tolerance might involve the induction of various protective pathways that could ensure a smooth transition and maintenance of the anoxic state through the suppression of cellular proliferation. In this thesis, I examined several pathways and proteins (primarily in Chapters 2 and Chapter 3) that could contribute to cell cycle regulation in T. scripta elegans. Furthermore, I expanded this study to include the transcriptional activity and post-transcriptional regulation of several critical components of cell cycle regulation under anoxia (primarily in Chapter 4 and Chapter 5).

\subsection{Regulation of the cell cycle though Cyclin D1 control}

In response to favorable conditions of cellular growth and proliferation, the cell cycle can be initiated through the activation of the PI3K/Akt pathway. Activation of the PI3K/Akt pathway can promote proliferation by inhibiting the protein kinase responsible for Cyclin D1 phosphorylation and subsequent degradation, GSK-3 $\beta$. GSK-3 $\beta$ is inhibited by the PI3K/Akt pathway through site-specific phosphorylation (Ser9) via Akt kinase. Interestingly, results from Chapter 2 indicate that in the anoxic red-eared slider turtle there is an increase in relative Akt activity in both kidney and white skeletal muscle 
tissues (Figure 2.5). Both tissues displayed a significant increase in relative Akt phosphorylation levels (both Thr308 and Ser473) in response to $20 \mathrm{~h}$ anoxia, indicating a relative increase in active Akt protein. Both tissues also showed an increase in the relative phosphorylation of GSK-3 $\beta$ (Ser9), suggesting that Akt is active and inhibiting GSK-3 $\beta$ function. Indeed the downstream phosphorylation of Cyclin D1 (Thr284) correspondingly decreased in response to anoxia in kidney. However, despite an inactivation of GSK-3 $\beta$ activity in white muscle tissue, the relative phosphorylation of Cyclin D1 and the ratio of total Cyclin D1 protein to p-Cyclin D1 (Thr284) did not significantly change (Figure 2.6 and Figure 5.4B). These results suggest that either (1) GSK-3 $\beta$ does not significantly regulate Cyclin D1 phosphorylation during normoxia, or (2) since skeletal muscle is typically post-mitotic, perhaps alternate (non-reversible) mechanisms of proliferation are controlling the cell cycle.

In liver tissue, the regulation of GSK-3 $\beta$ activity appears to differ from that of either kidney or white skeletal muscle. Despite what appears to be maintenance of both Akt and GSK-3 $\beta$ activities from normoxic to anoxic states, there is a relative decrease in Cyclin D1 phosphorylation similar to that seen in both kidney and white skeletal muscle (Figure 2.6 and Figure 5.4B). It is possible that GSK-3 $\beta$ activity is redirected during periods of anoxia from regulating Cyclin D1 to the regulation of other cellular processes, such as the inhibition of glycogen synthesis. Previous studies from the Storey lab have found that in response to anoxia, tolerant turtles are able to supply glucose to other organs by exploiting their large liver glycogen stores (Mehrani and Storey, 1995). Indeed, active GSK-3 $\beta$ would be able to contribute to this metabolic activity, by phosphorylating and inactivating glycogen synthase (at Ser641), the rate-limiting enzyme that promotes 
glycogen deposition. Indeed, GSK-3 was originally identified as a regulator of GS and is well-established in the literature to this effect (Patel et al., 2008). Inhibition of GSK-3 $\beta$ by Akt (via Ser9 phosphorylation) results in the dephosphorylation and activation of GS, leading to increased rates of glycogen synthesis (Eldar-Finkelman et al., 1996; Parker et al., 1983). The relative unchanged Akt activity between control and anoxia may also play a role in the regulation of cellular metabolism in anoxic turtle liver. One of the alternative roles of Akt is to regulate glucose metabolism, an important process as the liver is the main organ of glycogen synthesis and storage. Akt has been shown to indirectly activate the glycolytic rate-controlling enzyme, phosphofructokinase-1 (PFK1), by phosphorylating PFK2 (Franke et al., 2003). Active PFK2 produces fructose-2,6bisphosphate $\left(\mathrm{F} 2,6 \mathrm{P}_{2}\right)$, a potent allosteric activator of PFK1. Indeed, an increase in Akt signaling may facilitate an increase in anaerobic glycolysis in anoxic turtles in kidney and white skeletal muscle. Previous studies in the turtle have indicated the allosteric regulation of enzymes involved in glucose metabolism. These studies have clearly indicated glycolytic activation after $1 \mathrm{~h}$ of submergence from research on PFK1 enzyme kinetics (Storey and Hochachka, 1974; Kelly and Storey, 1988; Brooks and Storey, 1989). After one hour of submergence (early hypoxia), activation of glycolysis was seen in brain, heart and white skeletal muscle (Kelly and Storey, 1988). However, these same organs showed clear evidence that the previous glycolytic activation was reversed after five hours of submergence. Interestingly, this same study identified differential regulation of glycolysis in liver tissue, indicating a very rapid glycolytic inhibition occurring within the first hour of submergence. In contrast to kidney and white skeletal muscle, liver tissue did not show an increase in relative glycolytic activity and may be reflected in the 
inhibition of glycogen synthesis. This decrease may be facilitated by a decrease in Akt activity resulting in a reduction in $\mathrm{F} 2,6 \mathrm{P}_{2}$ levels and thereby allowing glycogenolysis to be directed towards the exportation of fermentable glucose fuel to other organs to satisfy substrate needs (Kelly and Storey, 1988).

As the regulation of Cyclin D1 protein is critical to the initiation of the cell cycle, the characteristics of this cyclin were explored to a much further extent than just an analysis of protein expression and phosphorylation alone. The expression of cyclin $d 1$ transcripts was measured to further determine possible modes of transcriptional, posttranscriptional or post-translational regulation. Despite a decrease in Cyclin D1 protein expression to some degree (both total and nuclear) and no increase in apparent proteasomal degradation (indicated by Tyr284 phosphorylation), no change in cyclin $d 1$ mRNA was observed in any studied tissue. This regulation pattern suggests a possible post-transcriptional mode of regulating Cyclin D1 expression during anoxia. It has been proposed that the $3^{\prime} \mathrm{UTR}$ of cyclin $d 1$ is critical in regulating translation due to a combination of several regulatory elements, including AREs and microRNA binding sites (Wiestner et al., 2007; Rimokh et al., 1994). Recent studies examining the expression of cyclin d1, with and without the 3' UTR, have demonstrated that deletion of the 3' UTR (and the regulatory sites located within) in human fibroblasts leads to a hyperproliferative state (Deshpande et al., 2009). This same study further demonstrated that cyclin $d 1$ translation is dependent on the presence of both an AU-rich region and microRNA binding sites. To determine the potential for a similar mode of regulation in $T$. scripta elegans, the 3' UTR was sequenced and the gene was examined for the presence of both AU-rich regions (Figure 5.5) and potential microRNA binding sites (Figures 5.6). 
Exploration of the turtle cyclin d1 3' UTR sequence yielded an AU-rich region containing four AUUUA repeats within a 273 nucleotide region. A number of studies have suggested that the presence of an AU-rich region within the 3' UTR of cyclin d1 leads to an increase in mRNA destabilization and therefore could contribute to a decrease in cyclin d1 mRNA and consequently a decrease in Cyclin D1 protein expression (Lin et al., 2008; Guo et al., 2005). However, results from the present study are not suggestive of mRNA destabilization; the expression of cyclin $d 1$ mRNA does not significantly change throughout the anoxic exposure (Figure 2.9). Indeed, other studies have shown that AUrich regions in the 3'UTR of cyclin $d l$ do not lead to destabilization of the mRNA, but instead yields a stabilizing effect (Deshpande et al., 2009). This finding agrees with the turtle study, as the presence of AU-rich regions did not suggest mRNA destabilization. Instead negative regulation of cyclin $d 1$ translation is likely imparted by the presence of a conserved microRNA binding site for $m i R-16-1$ and $m i R-15 a$. The expression profiles of both $m i R-16-1$ and $m i R-15 a$ were inversely correlated with the expression of Cyclin D1 protein in liver and kidney tissues (Figure 5.9 and Figure 5.10). This correlation may be indicative of microRNA-induced translational suppression of cyclin dl, where an increased expression of $m i R-16-1$ and $m i R-15 a$ led to a depression of mRNA translation in proliferative tissues (such as liver and kidney). In skeletal muscle, no significant changes were seen in either Cyclin D1 protein or microRNA expression. This provides an indication that change in Cyclin D1 protein levels may occur in a tissue specific manner, perhaps indicative of proliferative potential. Additionally, the imperfect binding between both miR-16-1 and miR-15a with cyclin $d 1$ mRNA suggest a mechanism for translational silencing and not degradation (Figure 5.7). This discovery provides a potential 
mechanism to decrease Cyclin D1 protein without decreasing the expression of cyclin $d 1$ mRNA, such as that seen in the anoxic turtle. Overall, it appears that Cyclin D1 is a highly regulated protein in the anoxic turtle. Control of Cyclin D1 protein expression during periods of anoxia is controlled primarily though decreased rates of translation facilitated through an AU-rich region and imperfect microRNA binding and translational inhibition. It could be hypothesized that an imperfect microRNA binding indicates possible mRNA storage and that an AU-rich region contributes to greater stability of the stored transcripts. Both of these possibilities are supported by findings of stable mRNA expression throughout the anoxic stress in all tissues. If true, this mechanism of $c y c l i n d 1$ regulation would allow rapid translation and re-initiation of the cell cycle upon re-entry into the normoxic state.

\subsection{Anoxia induced of cell cycle arrest mechanisms}

This thesis also assessed the expression of crucial Cyclins and Cdks involved in driving the cell cycle, as well as the activation of signaling pathways that control cell cycle initiation. The ordered progression through cell cycle phases is controlled by the sequential expression of Cyclins. Both Cyclin D1 and Cyclin E1 are highly expressed during the $\mathrm{G}_{1}$ phase of the cell cycle, while Cyclin A1 and Cyclin B1 are expressed during the $\mathrm{S}$ and $\mathrm{G}_{2}$ phases, respectively. In response to anoxia, there is a clear overall decrease in the relative expression of total and nuclear Cyclin and Cdk proteins in both turtle liver and kidney tissues. This may be controlled, in part, by an initial decrease in cell cycle signaling via either the Akt pathway (Chapter 2) or mature microRNA

(Chapter 5) and their downstream initial influences on Cyclin D1 protein expression. 
Negative regulation of Cyclin D1 expression would have downstream effects on the expression of subsequent Cyclins and Cdks.

Although the protein and mRNA expression of all Cyclins and Cdks displayed similar expression patterns within tissues of similar proliferative potential (liver and kidney vs. white skeletal muscle), the expression of selected Cyclins required further exploration. Unlike the expression of all other Cyclins, the nuclear expression of Cyclin A1 protein significantly increased after $5 \mathrm{~h}$ anoxia in all studied tissues and remained over-expressed after $20 \mathrm{~h}$ anoxia in liver and kidney. The typical nuclear function of Cyclin A1 protein is to create a complex with Cdk2 and activate the replication of DNA. However, alternate roles for the Cyclin A1/Cdk2 complex exist and may be utilized by the turtle during anoxia. Cyclin $\mathrm{A} 1 / \mathrm{Cdk} 2$ complex has been shown to phosphorylate E2F1 which inactivates DNA binding and transactivation by E2F1 (Kitagawi et al., 1995). Thus two mechanisms exist whereby Cyclin A1 is able to either activate (DNA replication) or inhibit (E2F1 phosphorylation) the progression of the cell cycle. Although further studies will be needed to determine the possibility of Cyclin A1/Cdk2 phosphorylation of E2F1 and its downstream effects on DNA binding in the anoxic turtle, results from Chapter 4 show that the DNA binding of E2F1 decreases in response to anoxia in liver and kidney tissues. Hence, it could be hypothesized that the decrease in E2F1 DNA binding during anoxia could be a result of Cyclin A1/Cdk2 phosphorylation. Additionally, the expression of Cyclin B1 protein is not similar to any other Cyclin measured. The over-expression of Cyclin B1 protein and mRNA may be a result of cell cycle independent transcription mediated by the p53 transcription factor. Indeed, previous studies exploring the regulation of p53 in anoxic T. scripta elegans have shown an 
increase in p53 transcriptional activity in liver and white skeletal muscle; its effects in kidney tissue were not explored (Zhang et al., 2013). Interestingly, although both the total levels of Cyclin B1 protein and mRNA increased in response to anoxia, the nuclear localization of Cyclin B1 was decreased.Previous studies have documented a cytoplasmic accumulation of Cyclin B1 protein during interphase of the cell cycle. Additionally, studies in hypoxic cell cycle arrest in D. melanogaster embryos reported a surprising increase in Cyclin B1 protein during a $\mathrm{G}_{1}$-like arrest of the cell cycle (Douglas, 2005). Although this finding is intriguing, it seems unlikely that Cyclin B1 would be translated during such a prolonged arrest of the cell cycle only to accumulate until the stress is removed, especially considering its usual rapid rate of degradation (Toyoshima et al., 1998). Alternate roles for Cyclin B1 may include an involvement in promoting cell cycle arrest while the protein is sequestered in the cytoplasm and may be dependent on relative phosphorylation of the protein. Indeed, Toyoshima et al. (1998) found that when cell cycle arrest was induced by DNA damage in HeLa cells, Cyclin B1 accumulated in the cytoplasm and that other mechanisms that are independent from Cyclin B1 downregulation, may exist to arrest cells in response to cellular stress. Additionally, another study reported that during a prolonged arrest, Cyclin B1/Cdc2 activity remains high for hours in the checkpoint-arrested state (Lindqvist et al., 2007).

Results presented in Chapter $\mathbf{2}$ indicated a general regulation of critical cell cycle components, namely Cyclins and Cdks, in addition to a possible regulation of a signaling cascade known to drive cell cycle progression. Although results indicated a slowing or general arrest of the cell cycle, this specific chapter did not provide any information regarding phase-specific regulation. It was the goal of Chapter $\mathbf{3}$ to develop the findings 
from Chapter 2 and profile possible cell cycle arrest mechanisms (those specific to either $\mathrm{G}_{0} / \mathrm{G}_{1}$ or $\mathrm{G}_{2}$ ) that might be involved in regulating the cell cycle during periods of anoxia in the turtle. Overall, the results presented in Chapter $\mathbf{3}$ showed that there was a clear activation of proteins involved in establishing and maintaining $\mathrm{G}_{0} / \mathrm{G}_{1}$ arrest, including CKIs (p16 and p27) and Chk2 proteins. Mechanisms involved in establishing $\mathrm{G}_{2}$ arrest (Chk1 and p21) were not activated in response to anoxia, further indicating a $G_{0} / G_{1}$ arrest mechanism.

In response to anoxia, Chk2 was only activated in liver and kidney (determined by relative changes in Chk2 auto-phosphorylation at residue Thr387). The activation of Chk2 can function to phosphorylate both Cdc25a and other downstream targets, such as p53, which functions to activate stress responsive pathways and induce $G_{1}$ arrest (Levine and Oren, 2009; Chehab et al., 1999). Indeed previous research on anoxia in red-eared sliders has indicated an activation of p53 in response to anoxia (Zhang et al., 2013). Interestingly, the study by Zhang and colleagues (2013) detailed the relative phosphorylation levels of p53 (on Ser20) by Chk2 which increased 2 to 3-fold in response to $20 \mathrm{~h}$ anoxia. This suggests a possible mechanism for Chk2 to regulate both down-regulation of $\mathrm{Cdc} 25 \mathrm{a}$ and the activation p53 function during anoxia in the turtle. Interestingly, downstream microRNA targets of p53 with important roles in cell cycle arrest, $m i R-16-1, m i R-15 a$ and $m i R-34 a$, were found to increase in response anoxia in this thesis (Chapter 5). It is intriguing to hypothesize that perhaps the activation of Chk2 contributes to the activation of p53 (Ser20) in the turtle and indirectly allows for the increased expression of microRNAs known to arrest the cell cycle.

To assess the relative expression and activity of $\mathrm{p} 16$, total protein levels were 
assessed in response to anoxia, as well as the relative levels of a phosphorylation site specific to active p16 protein (Thr145). Total protein levels of p16 increased significantly during anoxia in both liver (after $5 \mathrm{~h}$ anoxia) and kidney (after $20 \mathrm{~h}$ anoxia); no changes were detected in white skeletal muscle (Figure 3.7). Since p16 is known to target Cdk4 and Cdk6 exclusively, its upregulation during anoxia, coupled with the decreased expression Cyclin D1 in the liver and kidney (see Chapter 2, Figure 2.7), suggests that the $\mathrm{G}_{1}$ stage of the cell cycle is strongly targeted for suppression during anoxia in these tissues. No significant changes in either Cyclin D1 or p16 protein levels were detected in white skeletal muscle; this is likely a reflection of the senescent nature of skeletal myocytes and not an alternate mechanism of cell cycle regulation (Walsh and Perlman, 1997). Additionally, results show that p27 protein and transcript expression displays tissue specific regulation.

The relative inhibitory phosphorylation of p27 (Thr187) decreased in both liver and kidney tissue in response to 5 and $20 \mathrm{~h}$ anoxia. Interestingly, p27 is actively phosphorylated (at Thr187) and inhibited by the kinase action of Cyclin/Cdk complexes, such as Cyclin E1/Cdk2 and Cyclin A/Cdk2. A decrease in the relative phosphorylation level of p-p27 (Thr187) may be a result of a reduction in Cyclin/Cdk activity in response to anoxia, possibly arising due to the inactivation of Cdk proteins (Tyr14/Thr15; see Chapter 2, Figure 2.14). Indeed multiple studies have found that a decrease in Thr187 phosphorylation of p27 is indicative of strong cell cycle arrest (Roy et al., 2007; Besson et al., 2006). Taken together, these results suggest a role for $\mathrm{p} 27$-induced cell cycle regulation in liver tissue during anoxia. Furthermore, several studies have indicated that hypoxia-inducible factor $1 \alpha(\mathrm{HIF}-1 \alpha)$ plays an essential role in the adaptive response of 
cells to hypoxia (Aragones et al., 2009). Studies by Horree et al. (2008) have suggested that increases in HIF-1 $\alpha$ transactivation activity increase expression of p27 mRNA. Although there are currently no studies documenting the role of HIF-1 $\alpha$ in anoxic turtles, given the increased expression of p27 mRNA, it could be hypothesized that a HIF- $1 \alpha / \mathrm{p} 27$ interaction may play a role in connecting a hypoxia-triggered induction of cell cycle arrest with the reorganization of glycolysis in liver tissue.

Analysis of protein and mRNA expression, in conjunction with the analysis of the proteins actively bound to DNA-binding E2F proteins, provided insight into the regulatory mechanisms of anoxia induced cell cycle arrest. After $20 \mathrm{~h}$ of anoxia it was found that the nuclear levels of E2F1 significantly decreased in both liver and kidney tissues. Interestingly, the decrease in E2F1 protein was correlated with an increase in $\mathrm{E} 2 \mathrm{~F} 4$ protein in the same cellular fractions. Additionally, the $e 2 f 4$ transcripts also increased after $5 \mathrm{~h}$ of anoxia in liver and after $20 \mathrm{~h}$ in kidney. These findings suggest a mechanism whereby E2F4 may replace E2F1 on its DNA binding site during extended periods of anoxia. Indeed, the DNA binding activity of E2F1 was found to significantly decrease after $20 \mathrm{~h}$ of anoxia whereas E2F4 binding increased at the same time-point in liver tissues; similar responses were seen in kidney tissue after $5 \mathrm{~h}$ anoxia. Although these findings, along with those presented in Chapter 2, further suggest $\mathrm{G}_{1} / \mathrm{G}_{0}$ arrest during periods of anoxia, they fail to distinguish between $\mathrm{G}_{1}$ arrest and quiescence $\left(\mathrm{G}_{0}\right)$. To distinguish between these two types of cell cycle arrest, the anoxic regulation of Retinoblastoma proteins ( $\mathrm{Rb}$ and $\mathrm{p} 130)$ and the arrest-specific DNA binding protein complexes must be determined. 
Overall analysis of Retinoblastoma proteins and arrest-specific DNA binding complexes identified a possible cell cycle arrest mechanism for anoxic turtle tissues. Results suggested that $\mathrm{Rb}$ is active during anoxia and that acetylation at $5 \mathrm{~h}$ reduces overall phosphorylation of $\mathrm{Rb}$ at $20 \mathrm{~h}$ of anoxia, allowing $\mathrm{Rb}$ to remain in its active state in liver tissue. Similar results were found in kidney although Rb activation appeared to occur after just $5 \mathrm{~h}$ anoxia instead of after $20 \mathrm{~h}$ as seen in liver. The overall expression of p130 was found to increase in both total and nuclear fractions after $20 \mathrm{~h}$ for liver and after $5 \mathrm{~h}$ for kidney. Results correlated with an increase in mRNA expression with each respective tissue. Although protein expression, post-translation modification and subcellular localization provide an indication of the possible role of each protein, a novel in vitro technique was developed for this thesis to probe the type of DNA-binding complex that assembles during anoxia.

The development of the DNA-bound protein complex ELISA method outlined in this thesis was used to evaluate the composition and transcriptional activity of DNA binding complexes. Results from the DNA-bound protein complex ELISA experiments suggest that the liver is progressing through a state of $\mathrm{G}_{1}$ arrest (Figure 4.14). Of particular interest is the binding of either Rb or p130 to the E2F transcription factor, since this is the mechanism widely understood to be the critical difference between $G_{1}$ and $G_{0}$ arrest. Similar to other models of anoxia-induced cell cycle arrest, turtle displayed an increase in $\mathrm{Rb}$ complex formation after $20 \mathrm{~h}$ anoxia, while no significant complex formation was detected for $\mathrm{p} 130$. Given these results, it would seem likely that the liver of anoxic turtles may enter into a state of $\mathrm{G}_{1}$ arrest after $20 \mathrm{~h}$ of anoxia, via a mechanism similar to that seen in as hypoxia tolerant organisms, such as C. elegans and D. 
melanogaster (Kops et al., 2002; Douglas and Haddad, 2003). Given the small, yet significant, increases in p130 mRNA and protein after $20 \mathrm{~h}$ anoxia, it could also be hypothesized that $G_{1}$ arrest is a transition state while liver cells progress to a long term state of quiescence $\left(\mathrm{G}_{0}\right)$.

Analysis of the same proteins and processes in kidney gave similar results to those detected in liver tissue. However, uniquely different from liver, it appears that kidney may undergo a state of cell cycle arrest as early as $5 \mathrm{~h}$ of anoxia. Results from the DNA-bound protein complex ELISA experiments suggested that cells of the anoxic kidney entered into a state of overall $\mathrm{G}_{1}$ arrest during anoxia. However unlike liver tissue, results also suggested that entry into $\mathrm{G}_{1}$ arrest is temporary and quickly leads into a transition into quiescence $\left(\mathrm{G}_{0}\right)$. Analysis of the DNA-binding complex during early anoxia $(5 \mathrm{~h})$ showed an increase in proteins that comprise a $\mathrm{G}_{1}$ arrest complex; including Rb, E2F4, HDAC4 and Suv39H1 (Figure 4.14). Interestingly, later stages of anoxia displayed an increased presence of p130 in the arrest complex. It can be hypothesized that the increased presence of both $\mathrm{Rb}$ and p130 during later stages of anoxia could be an indication that kidney may be comprised either of two populations of cell-cycle arrest mechanisms (either $\mathrm{G}_{1}$ or $\mathrm{G}_{0}$ ), or that cells transition through $\mathrm{G}_{1}$ arrest in early stages of anoxia towards a sustained quiescence $\mathrm{G}_{0}$ for prolonged periods of arrest. This latter mechanism makes sense; it is well documented that cells exposed to various environmental stresses will respond by arresting cells at the R-point transition $\left(\mathrm{G}_{1}\right.$ arrest $)$ (Chiu et al., 2011). Additionally, $\mathrm{G}_{1}$ arrest at the R-point transition is necessary for entry into quiescence (Liu et al., 2007). Therefore, cell populations within the kidney of anoxic T. scripta elegans may appear to establish $\mathrm{G}_{1}$ arrest during early anoxia, while 
undergoing a transition to reversible quiescence $\left(\mathrm{G}_{0}\right)$ for long term suppression of the cell cycle during sustained anaerobiosis.

Although both liver and kidney showed regulation of cellular proliferation and displayed mechanisms of cell cycle arrest under anoxia, white skeletal muscle appears to forego these specific regulatory mechanisms. As skeletal muscle is primarily senescent, with irreversible mechanisms of cell cycle arrest already in place, perhaps small alterations in $\mathrm{Rb}$ and $\mathrm{E} 2 \mathrm{~F}$ regulation are needed to utilize specific pathways of the cell cycle without undergoing division. Several studies have documented that cellular hypertrophy, or growth without division, is an adaptive response to various physiological stimuli in post-mitotic muscle. These tissues typically display transient and selective transcriptional activity of E2F1, leading to the increased expression of a subset of genes necessary for muscle maintenance without cellular division. Such genes typically include those involved with DNA repair (including $p c n a$ ), protein synthesis,mitochondrial function and programmed cell death (Shin et al., 1995; Al-Musawi et al., 2011).

\subsection{Final Conclusions}

In conclusion, this thesis provides an in-depth analysis of the possible mechanisms contributing to cell cycle arrest during anoxia in the tolerant turtle, $T$. scripta elegans. Each chapter builds upon the general principles of cell cycle regulation to create an overview of the specific $\mathrm{G}_{1} / \mathrm{G}_{0}$ regulatory mechanisms used by the turtle to arrest the cell cycle during periods of anoxia. Results from Chapter $\mathbf{2}$ and Chapter $\mathbf{3}$ indicated a general regulation of critical cell cycle components within the $G_{1} / G_{0}$ phases of the cell

cycle, in addition to their possible regulation by signaling cascades (PI3K/GSK-3 $\beta$ and 
ATR/Chk2) that are known to regulate cell cycle progression in other situations.

Importantly, this thesis documented the extensive regulation of Cyclin D1 protein by (1) protein signaling, (2) post-translational modification (Chapter 2), (3) an AU-rich region, and (4) microRNA-induced translational suppression (Chapter 5). My study also successfully identified a phase-specific cell cycle arrest mechanism involving the Rb/E2F DNA-binding complex (Chapter 4). Overall, results from this thesis indicate that both kidney and liver enter into $\mathrm{a}_{1}$ arrest during anoxia. By contrast, the cell cycle in white skeletal muscle was found to be minimally regulated during anoxia and this finding is likely a reflection of its overall post-mitotic nature. Interestingly, kidney established a state of $\mathrm{G}_{1}$ arrest within $5 \mathrm{~h}$ anoxia and subsequently transitioned to a sustainable $\mathrm{G}_{0}$ arrest by $20 \mathrm{~h}$ anoxia. However, it appears that liver $\mathrm{G}_{1}$ arrest was not established until 20 $\mathrm{h}$ anoxia. Future studies will need to explore the regulation of the cell cycle in liver after longer periods of anaerobiosis to determine whether hepatocytes are also able to transition into $\mathrm{G}_{0}$ arrest in a manner similar to kidney tissue. 


\section{References}

Al-Musawi, S.L., Lock, F., Simbi, B.H., Bayol, S.A., and Stickland, N.C. 2011. Muscle specific differences in the regulation of myogenic differentiation in chickens genetically selected for divergent growth rates. Differentiation, 82(3), 127-135.

Besson, A., Gurian-West, M., Chen, X., Kelly-Spratt, K.S., Kemp, C.J., and Roberts, J.M. 2006. A pathway in quiescent cells that controls p27KIP1 stabililty, subcellular localization, and tumor suppression. Genes \& Dev., 20, 47-64.

Biggar, K.K., Kornfeld, S.F., and Storey, K.B. 2011. Amplification and sequencing of mature microRNAs in uncharacterized animal models using stem-loop RT-PCR. Anal. Biochem., 416, 231-233.

Brooks, S.P.J., and Storey, K.B. 1989. Regulation of glycolytic enzymes during anoxia in the turtle Pseudemys scripta. Am. J. Physiol., 257, R278-R283.

Chehab, N.H., Malikzay, A., Stravridi, E.S., and Halazonetis, T.D. 1999. Phosphorylation of Ser-20 mediates stabilization of human p53 in response to DNA damage. Proc. Natl. Acad. Sci. USA, 96, 13777-13782.

Chiu, J., Tactacan, C.M., Shi-Xiong, T., Lin, R.C.Y., Wouters, M.A., and Dawes, I.W. 2011. Cell cycle sensing of oxidative stress in Saccharomyces cerevisiae by oxidation of a specific cysteine residue in the transcription factor Swi6p. J. Biol. Chem., 286(7), 5204-5214.

Deshpande, A., Pastore, A., Deshpande, A.J., Zimmermann, Y., Hutter, G., Weinkauf, M., Buske, C., Hiddemann, W., and Dreyling, M. 2009. 3' UTR mediated regulation of the cyclin D1 proto-oncogene. Cell Cycle, 8(21), 3584-3592.

Douglas, R., and Haddad, G. 2003. Effect of oxygen deprivation on cell cycle activity: a profile of delay and arrest. J. Appl. Physiol., 94, 2068-2083.

Eldar-Finkelman, H., Argast, G. M., Foord, O., Fischer, E.H., and Krebs, E.G. 1996. Expression and characterization of glycogen synthase kinase-3 mutants and their effect on glycogen synthase activity in intact cells. Proc. Natl. Acad. Sci. USA, 93, 10228-10233.

Franke, T.F., Hornik, C.P., Segev, L., Shostak, G.A., and Sugimoto, C. 2003. PI3K/Akt and apoptosis: size matters. Oncogene, 22, 8983-8998.

Guo, Y., Harwalkar, J., Stacey, D.W., and Hitomi, M. 2005. Destabilization of cyclin D1 message plays a critical role in cell cycle exit upon mitogen withdrawal. Oncogene, 24, 1032-1042.

Horree, N., Gort, E., Van der Groep, P., Heintz, A., Vooijs, M., van Diest, P. 2008. Hypoxia-inducible factor 1 alpha is essential for hypoxic p27 induction in endometrioid endometrical carcinoma. J. Pathol., 214, 38-45.

Kelly, D.A., and Storey, K.B. 1988. Organ-specific control of glycolysis in the anoxic turtle. Am. J. Physiol. Regul. Physiol., 225(5), R774-R779. 
Kitagawa, M., Higashi, H., Suzuki-Takahashi, I., Segawa, K., Hanks, S.K., Taya, Y., Nishimura, S., and Okuyama, A. 1995. Phosphorylation of E2F-1 by cyclin Acdk2. Oncogene, 10(2), 229-236.

Kops, G., Medema, R., Glassford, J., Essers, M., Dijkers, P., Coffer, P., Lam, E., and Burgering, B. 2002. Control of cell cycle exit and entry by protein kinase Bregulated forkhead transcription factors. Mol. Cell. Biol., 22, 2025-2036.

Lindqvist, A., van Zon, W., Rosenthal, C.K., and Wolthuis, R.M.F. 2007. Cyclin B-Cdk1 activation continues after centrosome separation to control mitotic progression. PLoS Biol., 5(5), e123.

Lin, S., Wang, W., Wilson, G.M., Yang, X., Brewer, G., Holbrook, N.J., and Gorospe, M. 2000. Down-regulation of cyclin D1 expression by prostaglandin $A_{2}$ is mediated by enhanced cyclin D1 mRNA turnover. Mol. Cell. Biol., 20(21), 7903-7913.

Levine, A.J., and Oren, M. 2009. The first 30 years of p53: growing ever more complex. Nat. Rev. Cancer, 9(10), 749-58.

Liu, H., Adler, A.S., Segal, E., and Chang, H.Y. 2007. A transcriptional program mediating entry into cellular quiescence. PLoS Genet., 3(6), e91.

Patel, S., Doble, B.W., MacAulay, K., Sinclair, E.M., Drucker, D.J. and Woodgett, J.R. 2008. Tissue-specific role of glycogen synthase kinase $3 \mathrm{~b}$ in glucose homeostasis and insulin action. Mol. Cell. Biol., 28(20), 6314.

Parker, P. J., Caudwell, F.B., and Cohen, P. 1983. Glycogen synthase from rabbit skeletal muscle; effect of insulin on the state of phosphorylation of the seven phosphoserine residues in vivo. Eur. J. Biochem., 130, 227-234.

Rimokh, R., Berger, F., Bastard, C., Klein, B., French, M., Archimbaud, E., Rouault, J.P., Santa Lucia, B., Duret, L., and Vuillaume, M. 1994. Rearrangement of CCND1(BCL1/PRAD1) 3' untranslated region in mantle-cell lymphomas and $\mathrm{t}$ (11q13)-associated leukemias. Blood, 83, 3689-96.

Roy, S., Kaur, M., Agarwal, C., Tecklenburg, M., Sclafani, R.A., and Agarwal, R. 2007. P21 and p27 induction by silibinin is essential for its cell cycle arrest effect in prostate carcinoma cells. Mol. Cancer Ther., 6, 2696-2707.

Shin, E.K., Shin, A., Paulding, C., Schaffhausen, B., and Yee, A.S. 1995. Multiple change in E2F function and regulation occur upon muscle differentiation. Mol. Cell. Biol., 15(4), 2252-2262.

Storey, K.B. 1996. Metabolic adaptations supporting anoxia tolerance in reptiles: recent advances. Comp Biochem Physiol B, 113(1), 23-35.

Storey, K.B. 2007. Anoxia tolerance in turtles: metabolic regulation and gene expression. Comp. Biochem. Physiol. A., 147, 263-276.

Storey, K.B., and Hochachka, P.W. 1974. Enzymes of energy metabolism in a vertebrate facultative anaerobe, Pseudemys scripta: turtle heart phosphofructokinase. J. Biol. Chem., 249, 1417-1422. 
Toyoshima, F., Moriquchi, T., Wada, A., Fukuda, M., and Nishida, E. 1998. Nuclear export of cyclin B1 and its possible role in the DNA damage-induced G2 checkpoint. EMBO J., 17(10), 2728-2735.

Walsh, K., and Perlman, H. 1997. Cell cycle exit upon myogenic differentiation. Curr. Opin. Genet. Dev., 7(5), 597-602.

Wiestner, A., Tehrani, M., Chiorazzi, M., Wright, G., Gibellini, F., Nakayama, K., Liu, H., Rosenwald, A., Muller-Hermelink, H.K., Ott, G., Chan, W.C., Greiner, T.C., Weisenburger, D.D., Vose, J., Armitage, J.O., Gascoyne, R.D., Connors, J.M., Campo, E., Montserrat, E., Bosch, F., Smeland, E.B., Kvaloy, S., Holte, H., Delable, J., Fisher, R.I., Grogan, T.M., Miller, T.P., Wilson, W.H., Jaffe, E.S., and Staudt, L.M. 2007. Point mutations and genomic deletions in CCND1 create stable truncated cyclin D1 mRNAs that are associated with increased proliferation rate and shorter survival. Blood, 109, 4599-606.

Zhang, J., Biggar, K.K., and Storey, K.B. 2013. Regulation of p53 by reversible posttranscriptional and post-translational mechanisms in liver and skeletal muscle of an anoxia tolerant turtle, Trachemys scripta elegans. Gene, 513(1), 147-155. 
APPENDIX A

\section{LIST OF PUBLICATIONS}




\section{Publications (submitted and accepted)}

19. Tessier, S.N., Zhang, J., Wu, C.W., Biggar, K.K. and Storey, K.B. Regulation of Akt signaling during primate torpor in the hibernating grey mouse lemur, Microcebus murinus. BBA-Mol. Cell Res. Submitted.

18. Wu, C.W., Biggar, K.K., and Storey, K.B. MicroRNA regulation during dehydration in the Western clawed frog, Xenopus tropicalis. Gene. Submitted.

17. Biggar, K.K., Wu, C.W., Tessier, S.N., Zhang, J., Perret, M. and Storey, K.B. Primate torpor: Regulation of stress-activated proteins kinases in a hibernating grey mouse lemur, Microcebus murinus. PLOS ONE. Submitted.

16. Biggar, K.K., Dawson, N.J. and Storey, K.B. (2013) Proteomic characterization of fructose-1,6-bisphosphate aldolase from anoxia tolerance Trachemys scripta elegans: Bioinformatics and mass spectrometric exploration of enzymatic activity. PLoS ONE. Accepted.

15. Biggar, K.K., Kotani, E., Furusawa, T. and Storey, K.B. (2013) Expression of freeze responsive proteins, Fr10 and Li16, from freeze tolerant frogs enhances survival of BmN insect cells. FASEB J. doi:10.1096/fj.13-230573.

14. Shaffer, B.H., Minx, P., Warren, D.E., Shedlock, A.M., Thomson, R.C., Valenzuela, N., Abramyan, J., Badenhorst, D., Biggar, K.K., et al. (2013) The painted turtle genome: extreme physiological adaptations in a slowly evolving lineage. Genome Res. 14: R28.

13. Wu, C.W., Biggar, K.K. and Storey, K.B. (2013) Biochemical adaptations of mammalian hibernation: exploring the thirteen-lined ground squirrel as a perspective model for naturally induced reversible insulin resistance. Braz. J. Med. Biol. Res. 46(1): 1-13.

12. Zhang, J., Biggar, K.K. and Storey, K.B. (2013) Regulation of p53 by reversible post-transcriptional and post translational mechanisms in liver and skeletal muscle of anoxia tolerant turtle, Trachemys scripta elegans. Gene. 513(1): 147-155.

11. Biggar, K.K., Dawson, N. and Storey, K.B. (2012) Real-time protein unfolding: a method for determining the kinetics of native protein denaturation using a qRT thermocycler. Biotechniques. 53(4): 231-238

10. Biggar, K.K., Kornfeld, S.F. and Storey, K.B. (2012) Suppression of muscle disuse atrophy during mammalian hibernation: MicroRNA regulation in the skeletal muscle of Myotis lucifugus. Genom. Proteom. Bioinform. 10(5): 295-301.

9. Biggar, K.K., Kornfeld, S.F., Maistrovski, Y. and Storey, K.B. (2012) Differential expression of miRNAs in the intertidal snail Littorina littorea during freezing and anoxia in foot muscle and hepatopancreas. Genom. Proteom. Bioinform. 10(5): 302-309.

8. Maistrovski, Y., Biggar, K.K. and Storey, K.B. (2012) The conserved response of $\mathrm{HIF}-1 \alpha$ regulation in mammalian hibernators: examining the regulation of $\mathrm{HIF}-1 \alpha$ by long non-coding RNAs in the thirteen lined ground squirrel (Spermophilus 
tridecemlineatus) and little brown bat (Myotis lucifugus). J. Comp. Physiol. B, 182(6): 849-859

7. Biggar, K.K. and Storey, K.B. (2012) Evidence for cell cycle suppression and microRNA regulation of cyclin D1 during anoxia survival in turtles. Cell Cycle 11(9): 1705-1713

6. Biggar, K.K., Groom, A. and Storey, K.B. (2012) Hypometabolism in turtles: Physiological and molecular strategies of anoxia survival. In Hypometabolism: Strategies of survival in vertebrates and invertebrates (eds. Nowakowska, A. and Caputa, M.) Research Signpost. ISBN: 978-81-308-0471-2

5. Biggar, K.K., Kornfeld, S.F. and Storey, K.B. (2011) Amplification and sequencing of mature microRNAs in uncharacterized animal models using stem-loop reverse transcription-polymerase chain reaction. Anal. Biochem. 416(2):231-233

4. Roufayel, R., Biggar, K.K. and Storey, K.B. (2011) Regulation of cell cycle components during exposure to anoxic and dehydration stress in the wood frog, Rana sylvatica. J. Exp. Zool. 315A(8):487-494

3. Biggar, K.K. and Storey, K.B. (2011) The emerging roles of microRNAs in the molecular responses of metabolic rate depression. J. Mol. Cell Biol. 3(3): 167-175

2. Biggar, K.K., and Storey, K.B. (2009) Perspectives in cell cycle regulation: Lessons from an anoxic vertebrate. Current Genomics, 10(8): 573-584

1. Biggar, K.K., Dubuc, A., and Storey, K.B. (2009) MicroRNA regulation below zero: Differential expression of miRNA-21 and miRNA-16 during freezing in wood frogs. Cryobiology 59: 317-321

\section{Finished projects (not submitted)}

Biggar, K.K. and Storey, K.B. MicroRNA identification and regulation in the brain of hibernating little brown bats.

Biggar, K.K. and Storey, K.B. Indentification of mature microRNA and their expression in freeze tolerant hatchling painted turtles.

Biggar, K.K., Hapsodou, M., Yan, K. and Storey, K.B. Regulation of heat shock proteins during hibernation in the little brown bat, Myotis lucifugus.

Biggar, K.K. and Storey, K.B. Indentification of mature microRNA and their expression profile during anoxia in the tolerant turtle, Trachemys scripta elegans.

Biggar, K.K. and Storey, K.B. Regulation of cell cycle regulating proteins during anoxia in the tolerance red-eared slider turtle, Trachemys scripta elegans.

Biggar, K.K. and Storey, K.B. Anoxia responsive control of the cell cycle via the $\mathrm{G}_{1^{-}}$ arrest checkpoint and regulation of E2F1 and E2F4 during anoxia in the tolerant turtle, Trachemys scripta elegans.

Biggar, K.K. and Storey, K.B. Cell cycle control via regulation of Rb/E2F complex formation during anoxia in the tolerant turtle, Trachemys scripta elegans. 
Biggar*, K.K., Wu*, C.W. and Storey, K.B. MicroRNA regulation in the brain of hatchling painted turtles (Chrysemys picta marginata) during freezing.

Biggar*, K.K., Wu*, C.W., Zhang, J, Tessier, S.N., and Storey, K.B. Changes in gene expression during primate torpor: comparing warm and cold hibernators.

Wu, C.W., Tessier, S.N., Zhang, J., Biggar, K.K. and Storey, K.B. Responses of heatshock proteins during warm torpor in the grey mouse lemur, Microcebus murinus.

Wu, C.W., Biggar, K.K. and Storey, K.B. The translation state of differentially expressed mRNAs in the liver of hibernating 13-lined ground squirrel (Ictidomys tridecemlineatus).

Yan K., Biggar, K.K., Hapsodou, M. and Storey, K.B. Regulation of heat shock proteins during hibernation in the little brown bat.

Zayed, H., Biggar, K.K., Kruvorucho, A. and Storey, K.B. Regulation of the Akt signaling pathway in the anoxia tolerant turtle, Trachemys scripta elegans. 


\section{APPENDIX B}

\section{COMMUNICATIONS AT SCIENTIFIC MEETINGS}




\section{Inivited Lectures}

Biggar, K.K. Protein regulation in natural models of environmental stress tolerance. Invited departmental lecture. Schulich School of Medicine \& Dentistry, Department of Biochemistry. The University of Western Ontario, November $15^{\text {th }}$ 2012.

Biggar, K.K. Regualtion of metabolic rate depression by non-coding RNA: New insights into microRNA and long non-coding RNA in natural stress tolerance. Invited departmental lecture. Faculty of Medicine, Department of Cellular \& Molecular Medicine. The University of Ottawa, November $15^{\text {th }} 2012$.

Biggar, K.K. Carbohydrate metabolism and Lipids and Membranes. Course lecture(s). Introductory to Biochemistry. Carleton University, October $25^{\text {th }}$ and $27^{\text {th }} 2011$.

\section{Invited Presentations $(*$ - indicates presenting author)}

Biggar, K.K.*, and Storey, K.B. (2011) Novel mechanisms of cell cycle regulation during anoxic stress in an anoxic turtle, Trachemys scripta elegans. $8^{\text {th }}$ Annual Ottawa-Carleton Institute of Biology Research Conference, Carleton University, Ottawa, ON., Invited Oral Presentation

Biggar, K.K.* , and Storey, K.B. (2010) MicroRNA regulation of cyclin D1 during anoxic stress in an anoxic turtle, Trachemys scripta elegans. $5^{\text {th }}$ Annual Canadian Society for Life Science Research Conference, McGill University, Montreal, Que. Invited Oral Presentation

\section{Conference Presentations $(*$ - indicates presenting author)}

K.K. Biggar and K. B. Storey*. Life in the slow lane: microRNA regulation of cyclin D1 during anoxia stress in Trachemys scripta elegans. $1^{\text {st }}$ International Conference on Oxidative Stress in Aquatic Ecosystems. Los Cabos, Mexico, November 20-24, 2012.

K.K. Biggar and K.B. Storey*. Changes in the Rb-E2F pathway during anoxic stress of an anoxia tolerant turtle. $1^{\text {st }}$ International Conference on Oxidative Stress in Aquatic Ecosystems. Los Cabos, Mexico, November 20-24, 2012.

K.K. Biggar and K. B. Storey*. Life in the slow lane: microRNA regulation of cyclin D1 during anoxia stress in Trachemys scripta elegans. $1^{\text {st }}$ International Conference on Oxidative Stress in Aquatic Ecosystems. Los Cabos, Mexico. Poster presentation

K.K. Biggar and K.B. Storey*. Changes in the Rb-E2F pathway during anoxic stress of an anoxia tolerant turtle. $1^{\text {st }}$ International Conference on Oxidative Stress in Aquatic Ecosystems. Los Cabos, Mexico. Poster presentation

Biggar, K.K.*, Dawson, N. and Storey, K.B (2012). Novel measurements of the kinetics of native denaturation using an qRT-PCR machine and Sypro Orange fluorescent dye. $19^{\text {th }}$ Methods in Protein Structure Analysis, Ottawa, ON. Poster presentation 
Biggar, K.K* . and Storey, K.B (2011). Life in the slow lane: microRNA regulation of cyclin D1 during anoxia stress in Trachemys scripta elegans. $26^{\text {th }}$ Annual meeting, Federação de Sociedades de Biologia Experimental (FeSBE), Rio de Janeiro, Brazil. Poster Presentation

Luu, B.*, Biggar, K.K, and Storey, K.B. (2011) The role of the troponin-tropomyosin complex and dystrophin-related glycoproteins in the anoxic red eared slider turtle, Trachemys scripta elegans. $26^{\text {th }}$ Annual meeting, Federação de Sociedades de Biologia Experimental (FeSBE), Rio de Janeiro, Brazil. Poster presentation

Biggar, K.K.*, and Storey, K.B. (2011) Anoxia survival in turtles: Possible roles of microRNAs in regulating metabolic rate depression. $50^{\text {th }}$ Annual Canadian Society of Zoology Meeting, Ottawa, ON. Oral Presentation

Kornfeld, S.F.*, Biggar, K.K., and Storey, K.B. (2011) Suppression of muscle disuse atrophy during mammalian hibernation: MicroRNA regulation in the skeletal muscle of Myotis lucifigus. 50 ${ }^{\text {th }}$ Annual Canadian Society of Zoology Meeting, Ottawa, ON. Poster Presentation

Maistrovski, Y.*, Biggar, K.K., and Storey, K.B. (2011) Novel HIF-1 regulation by noncoding RNA in mammalian hibernators. $50^{\text {th }}$ Annual Canadian Society of Zoology Meeting, Ottawa, ON. Poster Presentation

Biggar, K.K.*, and Storey, K.B. (2010) MicroRNAs: small RNAs with a big impact in metabolic rate depression. $7^{\text {th }}$ Annual Ottawa-Carleton Institute of Biology Research Conference, University of Ottawa, Ottawa, ON. Poster Presentation

Biggar, K.K.*, and Storey, K.B. (2009) MicroRNAs: small RNAs with a big impact in metabolic rate depression. $12^{\text {th }}$ Annual Chemistry and Biochemistry Graduate Research Conference, Concordia University, Montreal, Que. Oral Presentation

Biggar, K.K.* , and Storey, K.B. (2009) Changes in the Rb-E2F pathway during anoxic stress of an anoxia tolerant turtle. $48^{\text {th }}$ Annual Canadian Society of Zoology Meeting. Toronto, ON. Poster Presentation.

Biggar, K.K.*, and Storey, K.B. (2009) Anoxic cell cycle regulation in an anoxia tolerant turtle. $34^{\text {th }}$ annual APICS/CIC Student Chemistry Conference. Antigonish, N.S. Poster Presentation

Biggar, K.K.* , and Storey, K.B. (2009) Changes in the ATM/ATR pathway during anoxic stress of an anoxia tolerant turtle. $6^{\text {th }}$ Annual Ottawa Carleton Institute of Biochemistry Symposium. Ottawa, ON. Poster Presentation

Biggar, K.K.*, and Pulsifer, M. (2009) Where are all the Wood Turtles? Factors affecting detectability in a large population of Glyptemys insculpta in Nova Scotia: A necessity for calculating population size. $48^{\text {th }}$ Annual Canadian Society of Zoology Meeting. Toronto, ON. Poster Presentation

Biggar, K.K.* and Pulsifer, M. (2008) Factors affecting detection on a population of wood turtles (Glyptemys insculpta) from the St. Mary's River watershed, Nova Scotia. St. Francis Xavier University Undergraduate Research Conference. Antigonish, N.S. Poster Presentation 
Pulsifer, M.*, Tingley, R., Biggar, K.K., Coulomb, M., and Burns, H. (2007) Wood Turtle project. Eastern Woodland Owner Conference. Guysborough County, N.S. Provincial Conference

Pulsifer, M.*, Tingley, R., and Biggar, K.K. (2007) A community approach to stewardship and conservation of a species at risk. Woodland Owner Conference. Springhill, N.S. Provincial Conference 


\section{APPENDIX C}

\section{LIST OF ANTIBODIES}


Table C1. Antibody information and suppliers.

\begin{tabular}{cccc|cccc}
\hline Antibody & Type & Company & Stock \# & Antibody & Type & Company & Stock \# \\
\hline $\mathrm{Rb}$ & Total & Cell Signal & 9300 & Cdk4 & Total & Abgent & Ab7520b \\
$\mathrm{Rb}$ & Ser780 & Cell Signal & 9300 & Cdk6 & Total & GenScript & A01264 \\
$\mathrm{Rb}$ & Ser608 & Santa Cruz & sc-19806 & Cdk2 & Total & Abcam & ab6538 \\
Rb & Lys873 & Cell Signal & 2525 & Cdc2 & Total & GenScript & A01295 \\
p130 & Total & Santa Cruz & sc-317 & Cdk & Tyr14/15 & Santa Cruz & sc-28435 \\
E2F1 & Total & Santa Cruz & sc-193 & p16 & Total & Santa Cruz & sc-468 \\
E2F4 & Total & Santa Cruz & sc-6851 & p16 & Ser152 & Abgent & Ab3184a \\
Cyclin D1 & Total & Santa Cruz & sc-717 & p21 & Total & GenScript & A01150 \\
Cyclin D1 & Thr286 & Cell Signal & 2921 & p21 & Thr146 & GenScript & A01160 \\
Cyclin E1 & Total & Santa Cruz & sc-198 & p27 & Total & GenScript & A00436 \\
Cyclin A1 & Total & U of Iowa & A16 & p27 & Tyr187 & GenScript & A00333 \\
Cyclin B1 & Total & U of Iowa & F2F4 & HDAC4 & Total & GenScript & A00338 \\
Cdc25a & Total & GenScript & A00446 & SUV39H1 & Total & Santa Cruz & sc-25366 \\
Cdc25c & Total & GenScript & A00433 & Dicer & Total & Santa Cruz & sc-25117 \\
Chk1 & Total & Cell signal & 2345 & Akt & Total & Santa Cruz & A00959 \\
Chk1 & Ser317 & Cell signal & 2344 & Akt & Thr308 & GenScript & A00275 \\
Chk1 & Ser345 & Cell signal & 2341 & Akt & Ser473 & GenScript & A00301 \\
Chk2 & Total & Cell signal & 2662 & GSK-3 3 & Total & GenScript & A00196 \\
Chk2 & Tyr68 & Cell signal & 2661 & GSK-3 & Ser9 & GenScript & A00193 \\
Chk2 & Tyr383 & Cell signal & 2668 & PCNA & Total & Santa Cruz & sc-7907 \\
ATM & Total & GenScript & A01222 & & & & \\
ATR & Total & GenScript & A01253 & & & & \\
\hline
\end{tabular}




\section{APPENDIX D}

\section{LIST OF IMMUNOBLOTTING CONDITIONS}


Table D1. Immunoblotting conditions used in to assess protein expression in this thesis.

\begin{tabular}{|c|c|c|c|c|c|c|c|}
\hline Antibody & Type & $\begin{array}{l}\text { Gel } \\
(\%)\end{array}$ & $\begin{array}{c}\text { MW } \\
\text { (kDa) }\end{array}$ & [Primary] & Reactivity & [Secondary] & Notes \\
\hline $\mathrm{Rb}$ & Total & 8 & 110 & $1: 1000$ & Anti-mouse & $1: 500$ & $1.5 \mathrm{~h}$ secondary \\
\hline $\mathrm{Rb}$ & Ser780 & 8 & 110 & $1: 1000$ & Anti-rabbit & $1: 2000$ & \\
\hline $\mathrm{Rb}$ & Ser608 & 8 & 110 & $1: 1000$ & Anti-rabbit & $1: 4000$ & \\
\hline $\mathrm{Rb}$ & Lys873 & 8 & 110 & $1: 1000$ & Anti-rabbit & $1: 4000$ & \\
\hline p130 & Total & 8 & 130 & $1: 1000$ & Anti-rabbit & $1: 4000$ & \\
\hline E2F1 & Total & 10 & 72 & $1: 1000$ & Anti-rabbit & $1: 4000$ & \\
\hline E2F4 & Total & 10 & 50 & $1: 1000$ & Anti-goat & $1: 8000$ & \\
\hline Cyclin D1 & Total & 12 & 38 & $1: 1000$ & Anti-rabbit & $1: 2000$ & \\
\hline Cyclin D1 & Thr286 & 12 & 38 & $1: 1000$ & Anti-rabbit & $1: 2000$ & \\
\hline Cyclin E1 & Total & 12 & 47 & $1: 1000$ & Anti-rabbit & $1: 4000$ & \\
\hline Cyclin A1 & Total & 10 & 54 & $1: 1000$ & Anti-mouse & $1: 4000$ & \\
\hline Cyclin B1 & Total & 10 & 48 & $1: 1000$ & Anti-rabbit & $1: 4000$ & \\
\hline Cdk4 & Total & 12 & 35 & $1: 1000$ & Anti-rabbit & $1: 4000$ & \\
\hline Cdk6 & Total & 12 & 35 & $1: 1000$ & Anti-rabbit & $1: 4000$ & \\
\hline Cdk2 & Total & 12 & 34 & $1: 1000$ & Anti-rabbit & $1: 4000$ & \\
\hline $\mathrm{Cdc} 2$ & Total & 12 & 34 & $1: 1000$ & Anti-rabbit & $1: 4000$ & \\
\hline $\mathrm{Cdk}$ & Tyr14/15 & 12 & 33 & $1: 1000$ & Anti-rabbit & $1: 4000$ & \\
\hline Cdc25a & Total & 10 & 65 & $1: 1000$ & Anti-rabbit & $1: 4000$ & \\
\hline $\mathrm{Cdc} 25 \mathrm{c}$ & Total & 10 & 60 & $1: 1000$ & Anti-rabbit & $1: 4000$ & \\
\hline Chk1 & Total & 10 & 56 & $1: 1000$ & Anti-rabbit & $1: 4000$ & \\
\hline Chk1 & Ser317 & 10 & 56 & $1: 1000$ & Anti-rabbit & $1: 4000$ & \\
\hline Chk1 & Ser345 & 10 & 56 & $1: 1000$ & Anti-rabbit & $1: 4000$ & \\
\hline Chk2 & Total & 10 & 62 & $1: 1000$ & Anti-rabbit & $1: 4000$ & \\
\hline Chk2 & Tyr68 & 10 & 62 & $1: 1000$ & Anti-rabbit & $1: 4000$ & \\
\hline Chk2 & Tyr383 & 10 & 62 & $1: 1000$ & Anti-rabbit & $1: 4000$ & \\
\hline ATM & Total & 8 & 350 & $1: 1000$ & Anti-rabbit & $1: 2000$ & $65 \mathrm{~min}$ run $/ 2 \mathrm{~h}$ transfer \\
\hline ATR & Total & 8 & 250 & $1: 1000$ & Anti-rabbit & $1: 2000$ & $65 \mathrm{~min}$ run $/ 2 \mathrm{~h}$ transfer \\
\hline p16 & Total & 15 & 16 & $1: 1000$ & Anti-rabbit & $1: 4000$ & 0.2 um PVDF \\
\hline p16 & Ser 152 & 15 & 16 & $1: 1000$ & Anti-rabbit & $1: 4000$ & 0.2 um PVDF \\
\hline p21 & Total & 15 & 21 & $1: 1000$ & Anti-rabbit & $1: 4000$ & 0.2 um PVDF \\
\hline p21 & Thr146 & 15 & 21 & $1: 1000$ & Anti-rabbit & $1: 4000$ & 0.2 um PVDF \\
\hline p27 & Total & 12 & 27 & $1: 1000$ & Anti-rabbit & $1: 4000$ & 0.2 um PVDF \\
\hline p27 & Tyr187 & 12 & 27 & $1: 1000$ & Anti-rabbit & $1: 4000$ & 0.2 um PVDF \\
\hline HDAC4 & Total & 8 & 140 & $1: 1000$ & Anti-rabbit & $1: 4000$ & \\
\hline SUV39H1 & Total & 10 & 48 & $1: 1000$ & Anti-goat & $1: 8000$ & \\
\hline Dicer & Total & 8 & 250 & $1: 1000$ & Anti-goat & $1: 8000$ & $2 \mathrm{~h}$ transfer \\
\hline Akt & Total & 10 & 60 & $1: 1000$ & Anti-rabbit & $1: 4000$ & \\
\hline Akt & Thr308 & 10 & 60 & $1: 1000$ & Anti-rabbit & $1: 4000$ & \\
\hline Akt & Ser473 & 10 & 60 & $1: 1000$ & Anti-rabbit & $1: 4000$ & \\
\hline GSK-3 $\beta$ & Total & 10 & 47 & $1: 1000$ & Anti-rabbit & $1: 4000$ & \\
\hline GSK-3 $\beta$ & Ser9 & 10 & 47 & $1: 1000$ & Anti-rabbit & $1: 4000$ & \\
\hline
\end{tabular}


APPENDIX E RNA INTEGRITY 

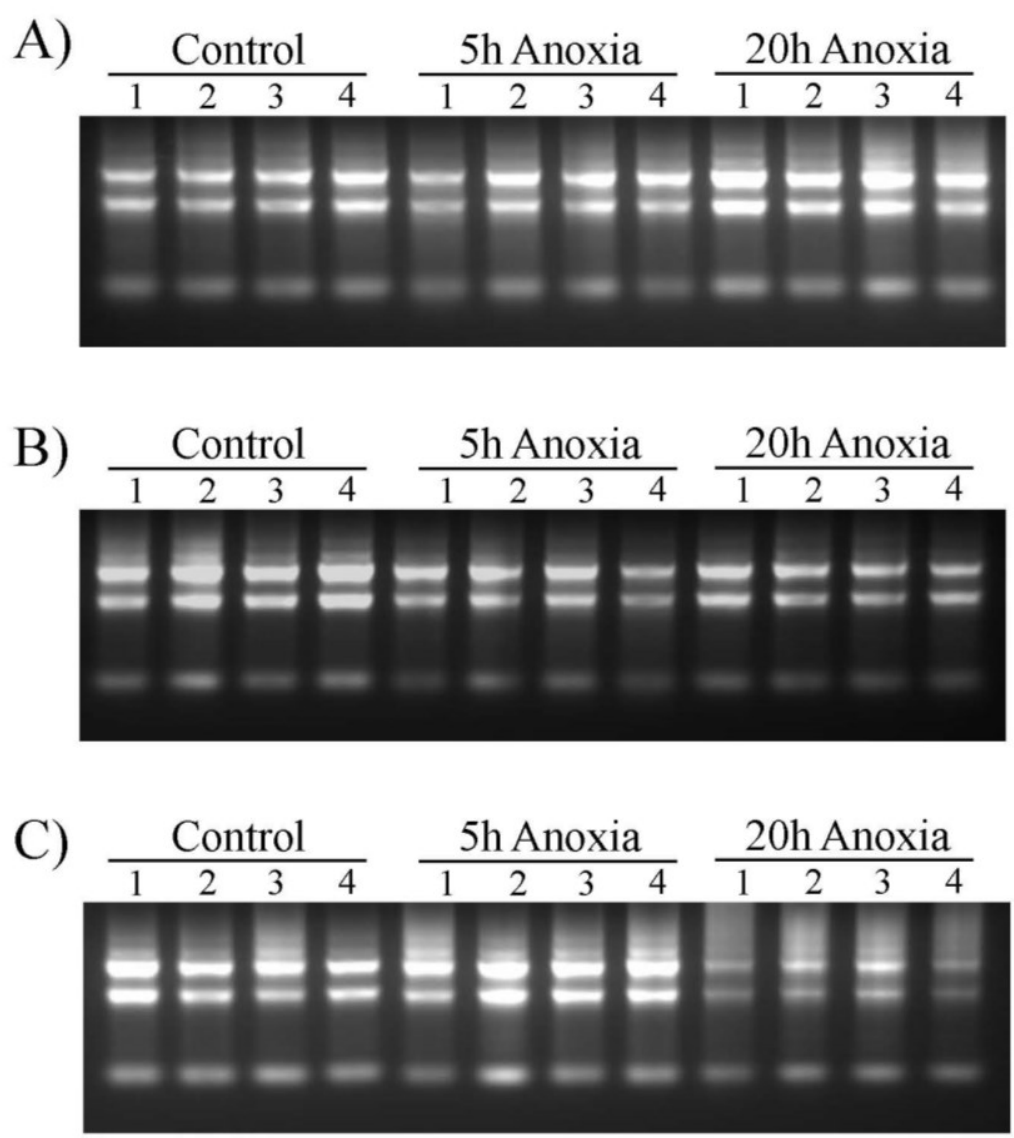

Figure E1. RNA integrity assessed by the presence of $18 \mathrm{~S}$ and $28 \mathrm{~S}$ rRNA bands from total RNA isolated from (A) liver, (B) kidney and (C) white skeletal muscle tissue from control and anoxic (5 and $20 \mathrm{~h}$ ) turtles, T. scripta elegans. 
APPENDIX F LIST OF PRIMERS 
Table F1. Primers used in this study.

\begin{tabular}{|c|c|c|}
\hline Target & Primer & Sequence \\
\hline \multirow[t]{3}{*}{ cyclin $d 1$} & Forward & $5^{\prime}$-ACTTGGATGCTGGAGGTTTG-3' \\
\hline & Reverse & $5^{\prime}$-AAGTCTGGGCATGTTTACGG-3' \\
\hline & 3' RACE & $5^{\prime}$-CCGTAAACATGCCCAGACTT-3' \\
\hline \multirow[t]{2}{*}{ cyclin el } & Forward & $5^{\prime}$-TGATCGGTTTATGGCAACAC-3' \\
\hline & Reverse & $5^{\prime}$-CAGAAGCGGCAAGTATTCCA-3' \\
\hline \multirow[t]{2}{*}{ cyclin al } & Forward & 5'-TTTCTTGCATGTCTGTCCTC-3' \\
\hline & Reverse & $5^{\prime}$-TTTSAGGAGCAGATGTTCCA-3' \\
\hline \multirow[t]{2}{*}{ cyclin bl } & Forward & $5^{\prime}$-CATGACTGCTGCCATCATTG-3' \\
\hline & Reverse & $5^{\prime}$-АСТCTCCACCATCAAGAACC-3' \\
\hline \multirow[t]{2}{*}{$c d k 4$} & Forward & $5^{\prime}$-ATGGAGAACTTCCAGAAGGT-3' \\
\hline & Reverse & 5' -CAGAGCTGCCTTTGCCGAAA-3' \\
\hline \multirow[t]{2}{*}{$c d k 6$} & Forward & $5^{\prime}-$ AAGGTGTTCAAGGCCCGCGA-3' \\
\hline & Reverse & 5' -GCTCTGGAACTTTATCCAAG-3' \\
\hline \multirow[t]{2}{*}{$c d k 2$} & Forward & $5^{\prime}$-GTGGARAAGATCGGRGAGGG-3' \\
\hline & Reverse & $5^{\prime}$-ACCATCTAGCAAARATGCA-3' \\
\hline \multirow[t]{2}{*}{$c d c 2$} & Forward & $5^{\prime}$-GGCCAAGAGTTACCTGTATC-3' \\
\hline & Reverse & $5^{\prime}$-CTGATTGGCTGGAAGATTGG-3' \\
\hline \multirow[t]{2}{*}{$p 27$} & Forward & $5^{\prime}$-CCTGGCTGGCAAGTTCGAGT-3' \\
\hline & Reverse & $5^{\prime}-\mathrm{ACCACCGAGGCATCCGATGA-3^{ \prime }}$ \\
\hline \multirow[t]{2}{*}{$r b$} & Forward & $5^{\prime}$-TGCAGATGGAAGATGACTTG-3' \\
\hline & Reverse & $5^{\prime}-$ TGTCCAACTGCTTCAGCAAA-3' \\
\hline \multirow[t]{2}{*}{ p130 } & Forward & $5^{\prime}$-GAAGAGGTMATGCCACCTCA-3' \\
\hline & Reverse & $5^{\prime}$-TCCAGRTGTCTGTCCATCAT-3' \\
\hline \multirow[t]{2}{*}{$e 2 f 4$} & Forward & $5^{\prime}$-AGAACAGYATYCAGTGGAAG-3' \\
\hline & Reverse & 5' -GTGAATCTGRWACTTCTTCT-3' \\
\hline \multirow[t]{2}{*}{ pcna } & Forward & $5^{\prime}$-TGCAGAGCATGGACTCCTCG-3' \\
\hline & Reverse & 5' -GGTCTGKGASAGCTTGATGT-3' \\
\hline \multirow[t]{2}{*}{$\alpha$-Tubulin } & Forward & $5^{\prime}-$ GGAAGATGCTGCCAATAACT-3' \\
\hline & Reverse & $5^{\prime}$-GTCTGGAACTCGGTCAGATC-3' \\
\hline
\end{tabular}




\section{APPENDIX G}

\section{LIST OF ANNEALING TEMPERATURES}


Table G1. Annealing temperatures used for the amplification of genes from this study.

\begin{tabular}{cc}
\hline Target & $\mathbf{T}_{\text {anneal }}\left({ }^{\circ} \mathbf{C}\right)$ \\
\hline cyclin $d 1$ & 62 \\
cyclin $e 1$ & 58 \\
cyclin $a 1$ & 57.5 \\
cyclin $b 1$ & 56 \\
cdk4 & 60 \\
cdk6 & 58 \\
cdk2 & 64 \\
cdc2 & 62 \\
p27 & 65 \\
$r b$ & 52.5 \\
p130 & 60 \\
e2f4 & 65 \\
pcna & 60 \\
$\alpha-T u b u l i n$ & 54 \\
\hline
\end{tabular}




\section{APPENDIX H}

\section{LIST OF MICRORNA AMPLIFICATION PRIMERS}


Table H1. Primers used in this study.

\begin{tabular}{lll}
\hline Target & Primer & Sequence \\
\hline \multirow{2}{*}{ miR-15-1 } & Stem-loop & 5'-CTCACAGTACGTTGGTATCCTTGTGATGTTCGATGCCATATTGTACTGTGAGCACAAACC-3' \\
& Forward & 5'-ACACTCCAGCTGGGTAGCAGCACATAATGG-3' \\
& Reverse & 5'-CTCACAGTACGTTGGTATCCTTGTG-3' \\
\multirow{2}{*}{$m i R-16 a$} & Stem-loop & 5'-CTCACAGTACGTTGGTATCCTTGTGATGTTCGATGCCATATTGTACTGTGAGCGCCAATA-3' \\
& Forward & 5'-ACACTCCAGCTGGGTAGCAGCACGTAAATA-3' \\
& Reverse & 5'-CTCACAGTACGTTGGTATCCTTGTG-3' \\
\multirow{2}{*}{$m i R-34 a$} & Stem-loop & 5'-CTCACAGTACGTTGGTATCCTTGTGATGTTCGATGCCATATTGTACTGTGAGAACAACCA -3' \\
& Forward & 5'-ACACTCCAGCTGGGTGGCAGTGTCTTAGCTG -3' \\
& Reverse & 5'-CTCACAGTACGTTGGTATCCTTGTG-3' \\
5S $r R N A$ & Stem-loop & 5'-CTCACAGTACGTTGGTATCCTTGTGATGTTCGATGCCATATTGTACTGTGAGAAAGCCTA -3' \\
& Forward & 5'-GGCCTGGTTAGTACTTGGATGG -3' \\
& Reverse & 5'-CTCACAGTACGTTGGTATCCTTGTG-3' \\
\hline
\end{tabular}




\section{APPENDIX I}

\section{ENSURING ANTIBODY SPECIFICITY FOR IMMUNOBLOTTING}


Throughout this thesis, several steps were taken to ensure that the correct band was quantified for western blotting. These include:

1. Blocking with milk before probing with the antibody was optimized to minimize any nonspecific binding and ensure that only one band appeared at the expected molecular weight.

2. Blots were run (at lease once) with a mammalian control homogenate in addition to the turtle samples to ensure that bands cross-react at the same molecular weight.

3. If antibodies were tested on several different animals by the company, antibodies that were shown to cross-react well with a wide number of different species were selected. For example, the histone $\mathrm{H} 3$ antibody used cross-reacted with animals ranging from mammals to fruit flies.

4. If antibodies for different regions of the protein were available from the company, the amino acid sequences of these regions were aligned with sequences from several vertebrates and antibodies specific to the best conserved regions were selected.

5. In cases where a partial turtle sequence was available prior to western blotting from PCR experiments, the translated turtle sequence was aligned with the sequence of the epitope the antibody was made against to assess conservation. Antibodies made from regions wellconserved with the turtle sequence were selected. 
APPENDIX J

\section{EXPERIMENTAL OPTIMIZATION FOR DNA-BINDING COMPLEX ELISA}




\section{Introduction to Method}

In order to assess the protein composition and DNA-binding ability of E2F complexes, a new technique combining the general technique of co-IP analysis with the benefits of a traditional transcription factor ELISA had to be created. This technique has the benefit of utilizing custom biotin labeled DNA probes made specific for the animal of interest. The use of custom DNA probes provides a benefit over traditional co-IPs which use capture antibodies, commonly derived from mammals, to purify protein complexes. This type of complex purification results in a high degree of false-positives in nonmammalian organisms often due to the use of polyclonal antibodies and high degree of non-specific cross-reactivity. However, DNA binding sites are highly specific for a particular transcription factor and are often conserved throughout much of evolution. It is based on this property that a co-IP based on DNA binding will provide a reliable purification of protein complexes with a relatively low degree of non-specific cross reactivity. The assay is based on the interaction between a streptavidin-coated microplate and a biotin-labeled probe containing the DNA-binding element of a transcription factor. This method allows the researcher to evaluate the composition of specific complexes and track any changes in protein composition throughout the development of a stress condition. The outlined method is completely novel as no other similar protocol can be found in peer-reviewed journals. However, this study merely builds upon existing techniques and protocols such as the preparation of nuclear homogenates for co-IPs and DNA binding affinity ELISAs. 


\section{Effect of ionic strength on protein binding}

To determine that ionic strength necessary for maximal binding of complex members without the binding of non-specific nuclear proteins, co-IP ELISAs were carried out at several ionic strength conditions approximate to physiological conditions. To determine the optimal strength of binding for both primary and secondary complex formation, the presence of both $\mathrm{E} 2 \mathrm{~F} 1$ and $\mathrm{Rb}$ were detected at several ionic strengths (Figure J.1A). All buffer conditions were tested with a low concentration of non-ionic detergent (0.05\% NP-40). In the liver, the E2F1 significantly bound to the DNA probe under all ionic strength condition, however, maximal binding was seen at an ionic strengths of $100 \mathrm{mM}(\mathrm{P}<0.005)$ and $140 \mathrm{mM}(\mathrm{P}<0.05)$, when compared to $80 \mathrm{mM}$ ionic strength, while minimal binding was seen at $80 \mathrm{mM}$. Primary complex formation of $\mathrm{Rb}$ was also highest at an ionic strength near physiological conditions of $100 \mathrm{mM}(\mathrm{P}<0.01)$ and $140 \mathrm{mM}(\mathrm{P}<0.01)$, when compared to $80 \mathrm{mM}$ ionic strength, while minimum binding was seen at $80 \mathrm{mM}$.

The specificity of the co-IP ELISA was determined by attempting to detect proteins that may non-specifically bind the DNA probe. For the purpose of this experiment nuclear proteins were selected to access non-specific binding in binding buffers at various ionic strengths. These proteins included one transcription factor (XBP1) and a structural protein (Troponin I) (Figure J.1B). In the liver, the XBP-1 did not significantly binding to the DNA probe under any ionic strength condition. However, non-specific binding of Troponin I was detectable below physiological ionic strength (< $100 \mathrm{mM})$ and decreased significantly above an ionic strength of $140 \mathrm{mM}(\mathrm{P}<0.05)$, compared to both 80 and $100 \mathrm{mM}$ values, to become indistinguishable from zero. 
A

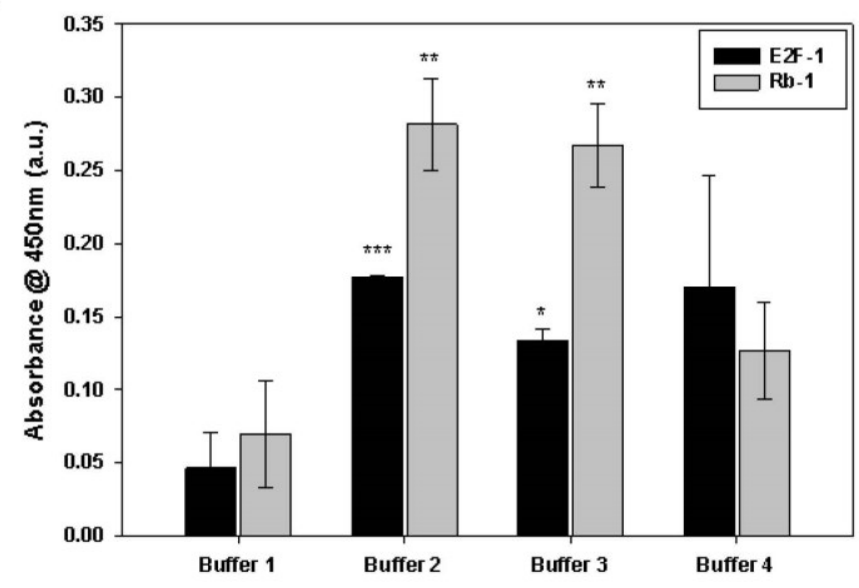

B

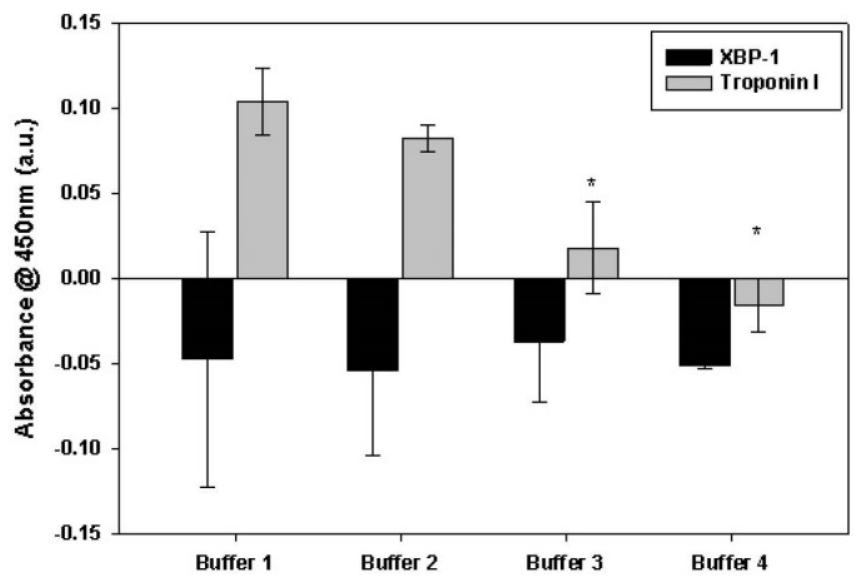

Figure J1. Effect of ionic strength (varied by $[\mathrm{NaCl}]$ ) on the relative binding of (A) E2F1 and $\mathrm{Rb}$ and (B) XBP-1 and Troponin I in liver from control ( $4{ }^{\circ} \mathrm{C}$ acclimated) red eared slider turtles, $T$. scripta elegans. Ionic strengths for representative buffers are; Buffer $1(80 \mathrm{mM})$, Buffer $2(100 \mathrm{mM})$, Buffer $3(140 \mathrm{mM})$ and Buffer $4(220 \mathrm{mM})$. Histogram shows normalized binding levels for various buffer ionic strengths and the influence on protein binding; data are means \pm SEM. $(n=3-4$ independent trials on tissue from different animals). Significant differences from the $80 \mathrm{mM}$ condition is indicated by $*(\mathrm{P}<0.05)$, $* *(\mathrm{P}<0.01)$ and $* * *(\mathrm{P}<0.005)$. 


\section{Effect of non-ionic detergent concentration on binding}

To determine the detergent strength necessary for maximal binding of complex members without the binding of non-specific nuclear proteins, co-IP ELISAs were carried out at several non-ionic detergent concentrations. To determine the optimal strength of binding for both primary and secondary complex formation, the presence of both E2F1 and Rb were detected at several concentrations of NP-40 (Figure J.2A). In the liver, the E2F1 significantly bound to the DNA probe under most non-ionic detergent conditions. No significant changes in E2F1 binding were found irrespective of detergent concentration or buffer ionic strength $(\mathrm{P}<0.05)$, however, a significant reduction of $\mathrm{E} 2 \mathrm{~F} 1$ binding was seen at $100 \mathrm{mM}$ ionic strength in an absence of NP-40. Additionally, E2F1 binding decreased significantly at $0.5 \% \mathrm{NP}-40$ in $140 \mathrm{mM}$ ionic strength buffer, compared to $0 \% \mathrm{NP}-40$ values. Primary complex formation of $\mathrm{Rb}$ was highest at a detergent concentration of $0.05 \%-0.5 \% \mathrm{NP}-40$ at $100 \mathrm{mM}$ and $0.05 \%$ at $140 \mathrm{mM}$ $(\mathrm{P}<0.05)$, compared to $0 \% \mathrm{NP}-40$ values. Binding of XBP-1 was not different than zero, while binding of Troponin I was only detectable between $0-0.05 \%$ NP-40 at $100 \mathrm{mM}$ ionic strength and $0 \% \mathrm{NP}-40$ at $140 \mathrm{mM}$ ionic strength when compared to all other values $(\mathrm{P}<0.05)$ (Figure J.2B). As a result of the low non-specific binding and high binding of both the transcription factor and primary binding partners, a binding buffer of $140 \mathrm{mM}$ and $0.05 \%$ NP-40 was used for all future quantification experiments. 
A

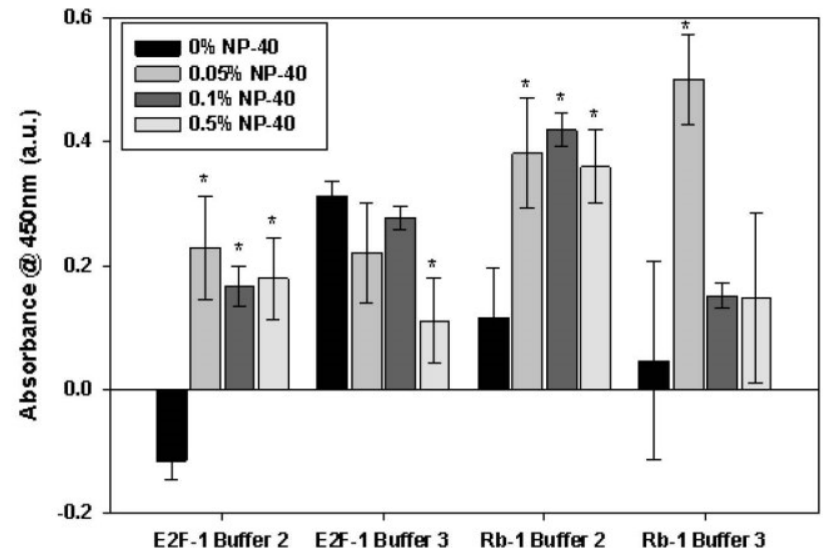

B

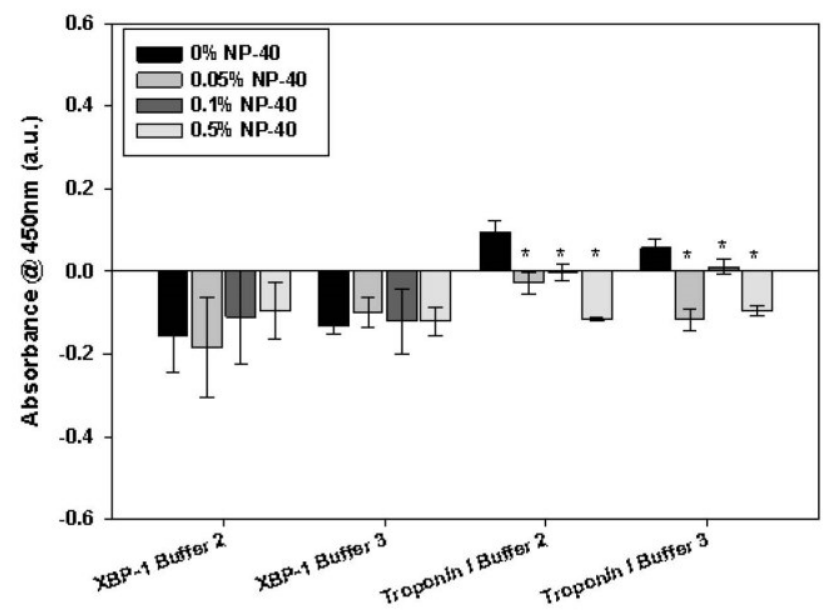

Figure J2. Effect of non-ionic detergent concentration (varied by \% NP-40) on the relative binding of (A) $\mathrm{E} 2 \mathrm{~F}$ and $\mathrm{Rb}$ and (B) XBP-1 and Troponin I in liver from control $\left(4^{\circ} \mathrm{C}\right.$ acclimated) red eared slider turtles, T. scripta elegans. Non-ionic detergent concentration was varied in two different ionic strength buffers; Buffer $2(100 \mathrm{mM})$ and Buffer $3(140 \mathrm{mM})$. Histogram shows normalized binding levels for various nonionic detergent concentrations and its influence on protein binding; data are means \pm SEM. $(n=3-4$ independent trials on tissue from different animals). * Indicates significant differences from the $0 \% \mathrm{NP}-40$ concentration in the respective buffer $(\mathrm{P}<0.05)$. 


\section{Effect of total protein concentration on binding}

Several concentrations of total protein extract were used to detect both primary and secondary complex structure. This experiment tests for any interaction between the ability of a protein to bind or be detected in the presence of high or low total protein concentration (Figure J.3). Maximum protein binding was seen between 8-32 $\mu \mathrm{g}$ of total protein for $\mathrm{E} 2 \mathrm{~F} 1$ when compared to $4 \mu \mathrm{g}$ total protein $(\mathrm{P}<0.05)$. Binding of $\mathrm{Rb}$ displayed a dose-response from $4 \mu \mathrm{g}$ to $16 \mu \mathrm{g}$ of total protein, when significant and maximal binding was achieved $(16-32 \mu \mathrm{g})(\mathrm{P}<0.05)$. 


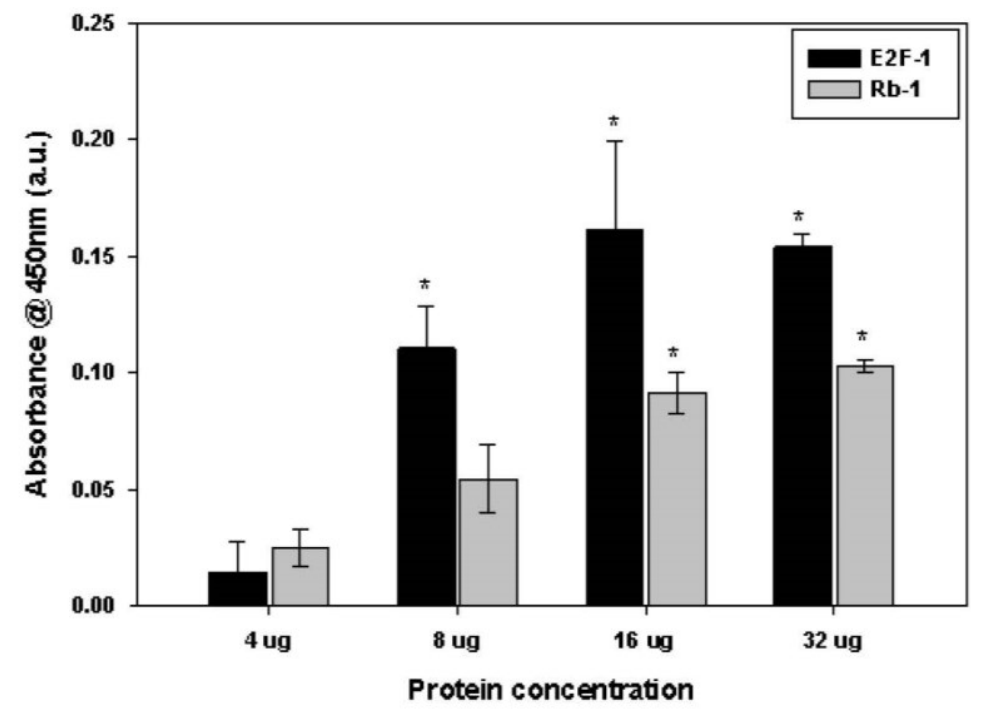

Figure J3. Effect of protein concentration on the relative binding of E2F1 and $\mathrm{Rb}$ in liver from control $\left(4{ }^{\circ} \mathrm{C}\right.$ acclimated) red eared slider turtles, T. scripta elegans. Histogram shows normalized binding levels for various total protein amounts and its influence on E2F1 and Rb binding; data are means \pm SEM. $(n=3-$ 4 independent trials on tissue from different animals). ${ }^{*}$ Indicates significant differences from the $4 \mu \mathrm{g}$ total protein $(\mathrm{P}<0.05)$. 


\section{APPENDIX K}

\section{EXPERIMENTAL METHOD AND VALIDATION OF MATURE MICRORNA AMPLIFICATION}




\section{Introduction to the Method}

MicroRNAs are increasingly being shown to play important roles in regulating the gene expression response by cells and organs to environmental stress. As such, it is critical that better methods be developed to allow discovery of miRNA species and their sequences in a wide variety of nontraditional model organisms. Unfortunately, the previously outlined method requires the use of specific TaqMan probes that need to be designed and optimized for each miRNA, thereby increasing the cost of the assay. Because PCR product amplification cannot be validated, this method is effectively limited to quantitative RT-PCR (qRT-PCR), where a melt curve analysis can be obtained, and to organisms whose miRNA sequences are already known.. Successful sequencing is required to validate the amplicon, ensuring accurate amplification of the targeted miRNA in new model organisms. In this thesis, I created an amplification method for miRNA RT-PCR analysis using stem-loop RT primers. This method uses a unique stem-loop adapter sequence to lengthen mature microRNA transcripts prior to amplification. Critically, this stem-loop adapter contains a specific sequence of nucleotides that yield a single, highly-stabile structure with a single predicted melting temperature, thereby increasing the specificity and efficiency of microRNA adaption and subsequence amplification and sequencing.

Together, this method allows the researchers to discover and evaluate the expression of novel miRNA sequences from diverse animal origins and validate amplification by sequencing the resulting PCR product. By allowing the sequencing of the PCR product, this ensures that amplification of the target mature miRNA sequences has indeed occurred. 


\section{This method has been published:}

Biggar, K.K., Kornfeld, S. and Storey, K.B. 2011. Amplification and sequencing of mature microRNAs in uncharacterized animal models using stem-loop RT-PCR. Anal. Biochem. 416, 231-233.

\section{Primer Design}

You will need the following primers in order to amplify a microRNA: miRspecific forward primer, miR-specific RT primer, and a universal primer. The first step is to obtain the mature microRNA sequence from the animal related closely to your own. These sequences can be obtained through the following website: http://www.mirbase.org/cgi-bin/browse.pl, simply select the organism and find your microRNA.

For example we can use gga-let-7a: 5'-TGAGGTAGTAGGTTGTATAGTT-3'

To Design the forward primer for this microRNA use the following protocol:

- This sequence has been pre-optimized for product size, primer pairing, $\mathrm{T}_{\mathrm{m}}$, and any homo or heterodimers that may occur.

- To design your forward primer simply replace the bases in red with the coordinating bases at the 5'-end of your microRNA. Use 15-17 bases depending a) the length of your microRNA and $b$ ) the matching of Tm with the universal primer:

\section{ACACTCCAGCTGGGXXXXXXXXXXXXXXX}

- So, for the gga-let-7a example we will have a forward primer sequence of: 
To Design the RT (Stem-loop) primer for this microRNA use the following protocol:

- The RT-primer does NOT need to adjusted and is a product of SPECIFIC base pairing and sequence to yield a primer of maximum length, of specific $T_{m}$, and will NOT form any other that the specific hairpin formation creating the specified stem-loop (exposing the microRNA binding sequence).

- To get your sequence take the last 8bp from the 3' end of your microRNA. So for ggalet-7a we the sequence: 5'-GTATAGTT-3'

- With this sequence, take the reverse complement sequence. This reverse complement sequence for gga-let-7a is: 5'-AACTATAC-3'

- Take the reverse complement sequence and replace the red X's in the following sequence with it. This will be the microRNA binding region of the StemLoop primer:

5'-CTCACAGTACGTTGGTATCCTTGTGATGTTCGATGCCATATTGTACTGTGAGXXXXXXXX-3'

- So, for the gga-let-7a example we will have a RC sequence of:

5'-TCACAGTACGTTGGTATCCTTGTGATGTTCGATGCCATATTGTACTGTGAGAACTATAC-3'

Universal Primer for PCR: 5'-CTCACAGTACGTTGGTATCCTTGTG-3' Universal Primer for Sequencing: 5'-CTCACAGTACGTTGG-3' 


\section{Mechanism of Amplification}

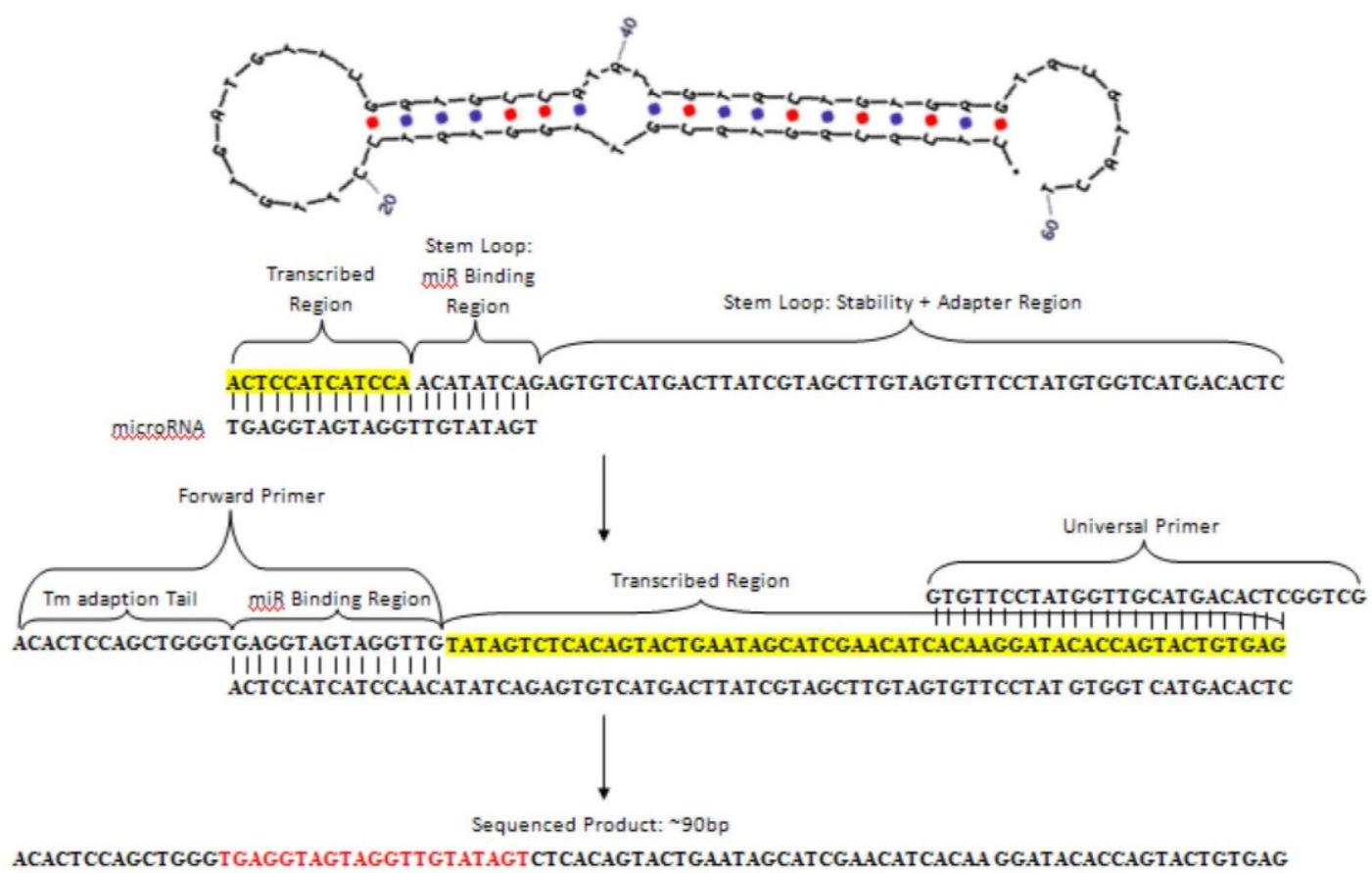

Figure K1. Depiction of the stem-loop RT-PCR protocol. Stem-loop RT primers bind to the 3' end of mature miRNAs and are reverse transcribed, creating miRNA-specific cDNA. The expression of mature miRNAs is then evaluated with RT-PCR using miRNA-specific forward and universal reverse primers 


\section{Method Validation}

The microRNA amplification protocol was validated by (1) comparing gelmigration patterns of turtle miR-1-1 to a synthetically produced sequence of miR-1-1 (Figure K.2) and (2) sequencing amplification products, ensuring proper and specific amplification of targets (Figure K.3). The PCR products ( $\sim 90 \mathrm{bp}$ ) were confirmed to encode the miR-1-1 sequences. Figure K.3A shows the significance of the shortened universal primer for sequencing reactions. Sequencing with a shortened universal reverse primer consistently yielded longer and more reliable sequencing results. Figure K.3B shows the mature miRNA sequence of miR-1-1 obtained from the RNA of $T$. scripta elegans (tsc), aligned with the sequences for chicken (gga), human (hsa), African clawed frog (xtr), fruit fly (dme), owl limpet (lgi), and zebrafish (dre).

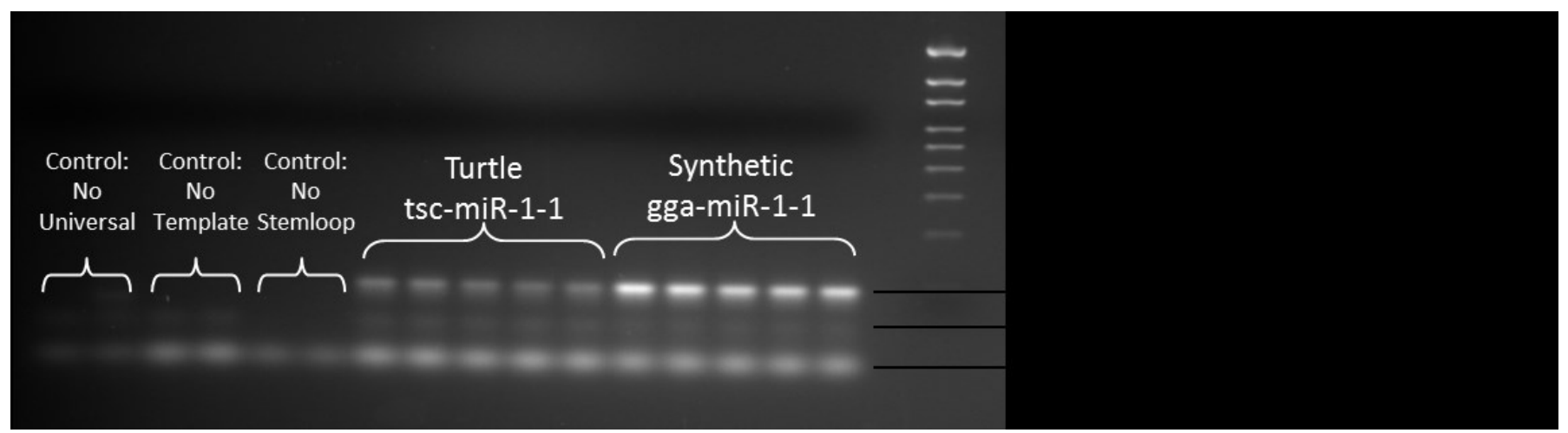

Figure K2. As a positive control, synthetic miR-1-1 oligonucleotides were amplified and sequenced using the modified stem-loop protocol. The PCR product from amplified synthetically produced miRNA oligonucleotides was used in the validation of the amplification of naturally occurring mature miRNAs from T. scripta elegans. 
A

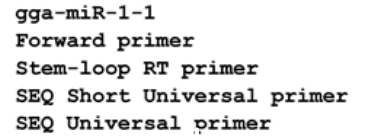

B

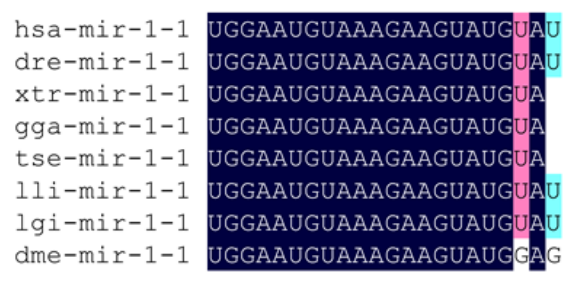

$\mathrm{C}$

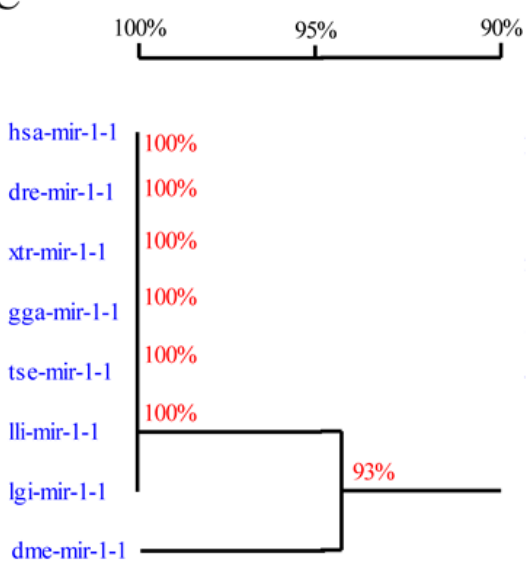

Figure K3. Conservation of mature miRNA sequences from T. scripta elegans (tsc). (A) Sequence results for tsc-miR-1-1, comparing both the experimental and modified universal primers. Shaded regions are indicative of base pair changes that are a result of erroneous sequencing. (B) Mature miRNA sequences (miR-1-1) from T. scripta elegans compared with respective mature miRNA sequences of human (hsa), zebrafish (dre), African clawed frog (xtr), chicken (gga), owl limpet (lgi), periwinkle snail (lli) and fruit fly $(d m e)$. Shaded residues show the relative degree of sequence conservation in the mature miRNA sequence between the vertebrate and invertebrate species. (C) Homology tree produced from miR-1-1 transcript alignments comparing $T$. scripta elegans with the respective mature miRNA sequences from the other six species. The percentage values correspond to the shared identity between the corresponding species. 